\title{
Um Modelo Semi-local para Folheações Riemannianas Singulares
}

\author{
Marcelo Kodi Inagaki
}

TESE APRESENTADA

$\mathrm{AO}$

Instituto DE MATEMÁticA E EstatísticA

DA

Universidade De SÃo Paulo

PARA

OBTENÇÃO DO TÍTULO

$\mathrm{DE}$

Doutor EM CIÊNCIAS

Programa: Matemática

Orientador: Prof. Dr. Marcos Martins Alexandrino da Silva

Durante o desenvolvimento deste trabalho o autor recebeu auxílio financeiro da CAPES e do $\mathrm{CNPq}$

São Paulo, setembro de 2020. 



\section{Um Modelo Semi-local para Folheações Riemannianas Singulares}

Esta versão da tese contém as correções e alterações sugeridas pela Comissão Julgadora durante a defesa da versão original do trabalho, realizada em 07/10/2020. Uma cópia da versão original está disponível no Instituto de Matemática e Estatística da Universidade de São Paulo.

Comissão Julgadora:

- Prof. Dr. Marcos Martins Alexandrino da Silva (orientador) - USP

- Dr. Mateus Moreira de Melo - USP

- Prof. Dr. Francisco Carlos Caramello Júnior - UFSC

- Prof. Dr. Llohann Dallagnol Sperança - UNIFESP

- Prof. Dr. Dirk Töben - UFSCar 



\section{Agradecimentos}

Agradeço, primeiramente, à minha família, meu pai Sérgio, minha mãe Tiyako e meu irmão Marcos. O apoio incondicional e a compreensão às decisões que me levaram a carreira acadêmica, bem como as privações, as dificuldades e as necessidades que essa escolha acarreta, é o que de mais próximo desta tese eu posso citar, dentre a infinidade de coisas pelas quais sou grato a vocês. Obrigado pelo amor, pela convivência, pela resiliência, enfim, por tudo.

Agradeço ao meu orientador, o professor Marcos, por me acolher após o mestrado e me conduzir ao estudo da teoria de folheações singulares, compartilhando sua maestria e sua paixão por esta área da geometria, da qual desconfio pertencer também. Sou grato, ainda, pelas conversas despropositadas sobre vários assuntos matemáticos e cotidianos, em encontros casuais pelo instituto, e por sempre esperar de mim, muito mais do que eu achava poder oferecer. Enfim, sou grato pela sua orientação.

Agradeço aos amigos do IME, por compartilharem o dia-a-dia, pelas conversas, quase sempre sobre matemática, e pela troca de experiências. Ao Fernando, o amigo com quem cursei boa partes das disciplinas do doutorado e com quem a relação de amizade mais se entrelaçou com os meus interesses em geometria. Ao Antonio, um dos pouquíssimos amigos com quem pude compartilhar muitos dos meus interesses pessoais para além da matemática e que muitas vezes me proporcionou um respiro do dia-a-dia, pretenciosas e ingênuas reflexões e muita cultura. Obrigado pela amizade.

Agradeço ao CNPq pelo apoio financeiro, sem o qual esta tese não seria possível. 


\section{Desenho}

Traça a reta e a curva, a quebrada e a sinuosa.

Tudo é preciso.

De tudo viverás.

Cuida com exatidão da perpendicular e das paralelas perfeitas.

Com apurado rigor.

Sem esquadro, sem nível, sem fio de prumo, traçarás perspectivas, projetarás estruturas. Número, ritmo, distância, dimensão.

Tens os teus olhos, o teu pulso, a tua memória.

Construirás os labirintos impermanentes que sucessivamente habitarás.

Todos os dias estarás refazendo o teu desenho.

Não te fatigues logo. Tens trabalho para toda a vida. E nem para o teu sepulcro terás a medida certa.

Somos sempre um pouco menos do que pensávamos. Raramente, um pouco mais.

(Cecília Meireles, 1963) 


\section{Resumo}

INAGAKI, M. K. Um modelo semi-local para folheações Riemannianas singulares. 2020. Tese (Doutorado em Ciências) - Instituto de Matemática e Estatística, Universidade de São Paulo, São Paulo, 2020.

O presente trabalho apresentará um modelo semi-local para folheações Riemannianas singulares. Mais precisamente, dada uma folheação Riemanniana singular $\mathcal{F}$ em uma variedade Riemanniana $(M, g)$, será provado que em uma vizinhança tubular saturada em torno de uma subvariedade $B$ fechada, saturada e contida em um estrato, a aproximação de primeira ordem de $\mathcal{F}$ (a folheação linearizada $\mathcal{F}^{\ell}$ ) que descreve parcialmente a dinâmica de $\mathcal{F}$, será dada pelas órbitas de um grupóide de Lie. Ainda, será provado que $\mathcal{F}$ e $\mathcal{F}^{\ell}$ são folheadamente difeomorfas a uma generalização da folheação de holonomia no fibrado normal a $B$.

Palavras-chave: Folheação Riemanniana Singular. Grupóide. Modelo Semi-local. 


\section{Abstract}

INAGAKI, M. K. A semi-local model for singular Riemannian foliations. 2020. Thesis (Doctor of Science) - Institute of Mathematics and Statistics, University of São Paulo, São Paulo, 2020 .

This work presents a semi-local model for a singular Riemannian foliation. More precisely, given a singular Riemannian foliation $\mathcal{F}$ in a Riemannian manifold $(M, g)$, it will be proved that in a saturated tubular neighbourhood around a closed saturated subamnifold $B$ in a stratum, the first order approximation of $\mathcal{F}$ (the linearized foliation $\mathcal{F}^{\ell}$ ) which partially describes the dynamic of $\mathcal{F}$ will be given by the orbits of a Lie groupoid. Moreover it will be proved that $\mathcal{F}$ and $\mathcal{F}^{\ell}$ are foliated diffeomorphic to a generalization of the holonomy foliation in the normal bundle of $B$.

Keywords: Singular Riemanninan Foliation. Groupoid. Semi-local Model. 


\section{Sumário}

Introdução $\quad$ xi

1 Folheações Singulares 1

1.1 Folheações e aplicações folheadas . . . . . . . . . . . . . . . . . . . 1

1.1.1 Construções básicas e exemplos de folheações singulares . . . . . . . . . . 3

1.1.2 Seções transversais locais . . . . . . . . . . . . . . . . . . . . 13

1.1.3 Subfolheações e subvariedades folheadas . . . . . . . . . . . . . . . . . . . 14

1.1.4 Campos verticais e folheados . . . . . . . . . . . . . . 18

1.2 Órbitas de campos e o teorema de Stefan-Sussmann . . . . . . . . . . . . . . . 19

2 Folheações Riemannianas Singulares (FRS) 23

2.1 Folheações Riemannianas singulares . . . . . . . . . . . . . . . . . . . . . 23

2.1 .1 Construções básicas e exemplos de FRS . . . . . . . . . . . . . . . . . 24

2.1.2 Seções normais locais e vizinhanças tubulares normais (VTN) . . . . . . . 30

2.1 .3 Dimensão das folhas e estratificação . . . . . . . . . . . . . . . 32

2.2 Homotetia, linearização de campos e folheação linearizada . . . . . . . . . . . . . 33

2.3 Folheação infinitesimal . . . . . . . . . . . . . . . . . . . . . 43

2.4 Folheações de tipo órbita e folheação de fecho local . . . . . . . . . . . . . . . . 47

3 Grupóides de Lie $\quad 49$

3.1 Grupóides de Lie e morfismo de grupóides de Lie . . . . . . . . . . . . . . . 49

3.2 Exemplos de grupóides de Lie . . . . . . . . . . . . . . . . . . . . . 52

3.3 Construção de grupóides . . . . . . . . . . . . . . . . . . . 57

3.3 .1 Ação de grupóides e grupóide de ação . . . . . . . . . . . . . . . . 57

3.3 .2 Ação de grupos sobre grupóides . . . . . . . . . . . . . . . . . . 60

4 Descrição Semi-local de uma FRS $\quad 67$

4.1 Distribuições semi-locais de uma FRS . . . . . . . . . . . . . . . . . . . 69

4.2 Subfolheações de holonomia semi-locais (Teorema A) . . . . . . . . . . . . 74

4.2.1 Construções preliminares à prova do Teorema A . . . . . . . . . . . 75

4.2 .2 Teorema A . . . . . . . . . . . . . . . . . . . 82

4.3 Folheação linearizada $($ Teorema B) . . . . . . . . . . . . . . . . . 83

4.3.1 Construções preliminares à prova do Teorema B . . . . . . . . . . . 85

4.3 .2 Teorema B . . . . . . . . . . . . . . . . . . . 90

4.4 Um modelo semi-local (Teorema C) . . . . . . . . . . . . . . . . . . 91

4.4.1 Folheação de holonomia generalizada (modelo semi-local) . . . . . . . . . 91

4.4 .2 Teorema C . . . . . . . . . . . . . . . . . . . . . . 94 
$\mathbf{x}$

Referências Bibliográficas 


\section{Introdução}

Uma folheação singular é, grosso modo, uma partição de uma variedade em subvariedades imersas e conexas, ditas as folhas da folheação, de modo que o plano tangente das mesmas seja localmente gerado por campos vetoriais tangentes à todas as folhas da folheação. Uma folheação singular, cujas folhas tenham a mesma dimensão, é dita uma folheação regular, ou simplesmente, uma folheação. Esta definição foi apresentada por Molino, em [23], e é derivada dos trabalhos de Stefan e Sussmann ([31] e [32]), onde os autores apresentam resultados a cerca da regularidade de órbitas de conjunto de campos vetoriais locais. Uma consequência destes resultados, obtida ainda nestes trabalhos e, por exemplo, em [20], é um tipo de teorema de Frobenius para distribuições singulares (distribuições com posto variável), exigindo-se de tais distribuições algo a mais além da involutividade presente no teorema clássico de Frobenius para distribuições regulares.

Uma folheação, em um ambiente Riemanniano, é dita Riemanniana se as geodésicas ortogonais a uma folha permanecem ortogonais a todas as outras folhas que elas encontram. Este conceito foi apresentado por Molino, em [23], onde o autor se baseou no trabalho de Bolton, em [9], a cerca de sistemas transnormais.

Um dos principais problemas na área de folheações Riemannianas singulares (FRS), até pouco tempo, foi a conjectura de Molino (proposta por Molino em [23]). Essencialmente, afirmase nesta conjectura que, dada $\mathcal{F}$ uma FRS de uma variedade Riemanniana $M$, a partição $\overline{\mathcal{F}}$ dada pelo fecho das folhas de $\mathcal{F}$ é uma FRS. O próprio Molino apresentou uma prova desta conjectura para o caso regular, em [23], e sugeriu uma idéia de prova para o caso de FRS de tipo órbita, em [24], que veio a ser formalizada por Alexandrino e Radeschi, em [8]. Antes disto, Alexandrino havia apresentado uma prova para o caso de FRS polares, em [2], e juntamente com Lytchak havia apresentado uma prova para o caso de FRS infinitesimalmente polares, em [6]. Mas, apenas recentemente, Alexandrino e Radeschi, em [7], provaram a conjectura. Neste trabalho, os autores descreveram a dinâmica da folheação linearizada $\mathcal{F}^{\ell}$ (a maior subfolheação de $\mathcal{F}$ transversalmente homogênea) e da folheação de fecho local $\mathcal{F}^{\text {lc }}$ (o fecho transversal da folheação $\mathcal{F}^{\ell}$ ) que, em um certo sentido, podem ser vistas como aproximações de primeira ordem da folheação singular $\mathcal{F}$, por meio da linearização de campos tangentes à $\mathcal{F}$. A relevância de tais folheações singulares, tanto na prova da conjectura de Molino, quanto na teoria de FRS, se deve ao fato de ambas descreverem, em parte, a dinâmica do fecho de $\mathcal{F}$, o que pode ser parcialmente visto no diagrama a seguir.

$$
\begin{aligned}
& \mathcal{F} \geqslant \mathcal{F}^{\ell} \leqslant \mathcal{F}^{\text {lc }} \\
& \mathbb{\wedge} \\
& \overline{\mathcal{F}} \geqslant \overline{\mathcal{F}^{\ell}}=\overline{\mathcal{F}^{1 \mathrm{c}}} .
\end{aligned}
$$

Entre outras coisas, a partir da descrição da dinâmica do fecho de $\mathcal{F}$, presente em [7], é possível intuir que as folheações singulares $\mathcal{F}^{\ell}$ e $\mathcal{F}^{\text {lc }}$ são dadas por órbitas de grupóides de Lie, 
ao menos em vizinhanças tubulares geométricas adequadas. É precisamente este o conteúdo de dois dos resultados deste texto. O primeiro (Teorema $\mathrm{A}$ ou, mais à frente no texto, Teorema 4.12), que versa sobre a folheação de holonomia em um fibrado vetorial, surge como consequência da demonstração concebida para o segundo (Teorema $\mathrm{B}$ ou, mais à frente no texto, Teorema 4.19), que versa sobre as folheações singulares $\mathcal{F}^{\ell}$ e $\mathcal{F}^{\text {lc }}$.

Teorema A. Sejam $B$ uma variedade, $\mathcal{F}_{B}$ uma folheação (regular) densa de $B, E$ um fibrado vetorial sobre $B$, com projeção $\pi, \nabla: \mathfrak{X}\left(\mathcal{F}_{B}\right) \times \Gamma(E) \longrightarrow \Gamma(E)$ uma conexão parcial linear e $\langle\cdot, \cdot\rangle \in \Gamma\left(E^{*} \otimes E^{*}\right)$ um produto interno compatível com $\nabla$. Considere, para cada $x \in E$,

$$
L_{x}:=\left\{\mathrm{P}_{\alpha}(x) \in E: \alpha \in C_{\text {par. }}^{\infty}\left(\mathcal{F}_{B}\right) \text { e } \alpha(0)=\pi(x)\right\}
$$

Então a partição $\mathcal{F}^{\nabla}:=\left\{L_{x}\right\}_{x \in E}$ é uma folheação singular, dada por órbitas de um grupóide de Lie.

Teorema B. Sejam $(M, g)$ uma variedade Riemanniana, $\mathcal{F}$ uma FRS e $B \subset M$ uma subvariedade fechada que é união de folhas de mesma dimensão. Então, existe uma vizinhança tubular geométrica, em torno de $B$, tal que $\mathcal{F}^{\ell}$ (resp. $\mathcal{F}^{\text {lc }}$ ) é dada por órbitas de um grupóide de Lie.

Ao contrário do que ocorre no caso regular, no qual a folheação pode ser localmente (em vizinhanças de pontos) descrita por submersões ou atlas folheados, como pode ser visto, por exemplo, em [10] e [23], no caso singular não há, ainda, uma descrição local conhecida. Neste texto, através do Teorema C, serão explicitadas descrições semi-locais de FRS, de suas linearizações e de seus fechos locais, em vizinhanças tubulares geométricas em torno de folhas fechadas. Isto será feito por meio da construção de uma folheação modelo em um fibrado vetorial (brevemente apresentada a seguir) e de um difeomorfismo folheado entre este modelo e a folheação singular, em uma vizinhança tubular geométrica. Mais a frente no texto (Teorema 4.24), será apresentado um caso mais geral do Teorema C, no qual as folhas fechadas serão substituídas por subvariedades mais gerais, assim como a folheação modelo.

\section{Modelo semi-local) Sejam}

- $B$ uma variedade Riemanniana,

- $E$ um fibrado vetorial Euclideano sobre $B$ (i.e. um fibrado vetorial com um produto interno em suas fibras), com projeção $\pi$,

- $\nabla: \mathfrak{X}(B) \times \Gamma(E) \longrightarrow \Gamma(E)$ uma conexão linear métrica (i.e. compatível com o produto interno das fibra), cujo operador de transporte paralelo, ao longo de uma curva suave por partes $\alpha \in C_{\text {par. }}^{\infty}(B)$, será denotado por $\mathrm{P}_{\alpha}$,

- $\mathcal{F}^{E}=\left\{L_{x}^{E}\right\}_{x \in E}$ uma folheação singular de $E$, tal que

$$
-L_{0_{b}}^{E}=\left\{0_{b}\right\}, \text { para todo } b \in B,
$$


- $L_{x}^{E} \subset E_{\pi(x)}$, para todo $x \in E$,

- $\mathrm{P}_{\alpha}$ leva folhas de $E_{\alpha(0)}$ em folhas de $E_{\alpha(1)}$, para toda $\alpha \in C_{\text {par. }}^{\infty}(B)$.

Dado $b \in B$, considere $\mathrm{K}_{b}^{0} \subset$ Iso $\left(E_{b}\right)$ o maior subgrupo de Lie conexo que fixa as folhas de $\mathcal{F}^{E}$ contidas em $E_{b}$.

Defina, para cada $x \in E$,

$$
\begin{aligned}
& L_{x}=\left\{\mathrm{P}_{\alpha}(y) \in E: y \in L_{x}^{E} \text { e } \alpha \in C_{\text {par. }}^{\infty}(B): \alpha(0)=\pi(x)\right\}, \\
& L_{x}^{\ell}=\left\{\mathrm{P}_{\alpha}\left(k_{\pi(x)} \cdot x\right) \in E: k_{\pi(x)} \in \mathrm{K}_{\pi(x)}^{0} \text { e } \alpha \in C_{\text {par. }}^{\infty}(B): \alpha(0)=\pi(x)\right\}, \\
& L_{x}^{\text {lc }}=\left\{\mathrm{P}_{\alpha}\left(\bar{k}_{\pi(x)} \cdot x\right) \in E: \bar{k}_{\pi(x)} \in \overline{\mathrm{K}}_{\pi(x)}^{0} \text { e } \alpha \in C_{\text {par. }}^{\infty}(B): \alpha(0)=\pi(x)\right\} .
\end{aligned}
$$

Então $\mathcal{F}(\nabla)=\left\{L_{x}\right\}_{x \in E}, \mathcal{F}^{\ell}(\nabla)=\left\{L_{x}^{\ell}\right\}_{x \in E}$ e $\mathcal{F}^{\text {lc }}(\nabla)=\left\{L_{x}^{\text {lc }}\right\}_{x \in E}$ são folheações singulares (modelos).

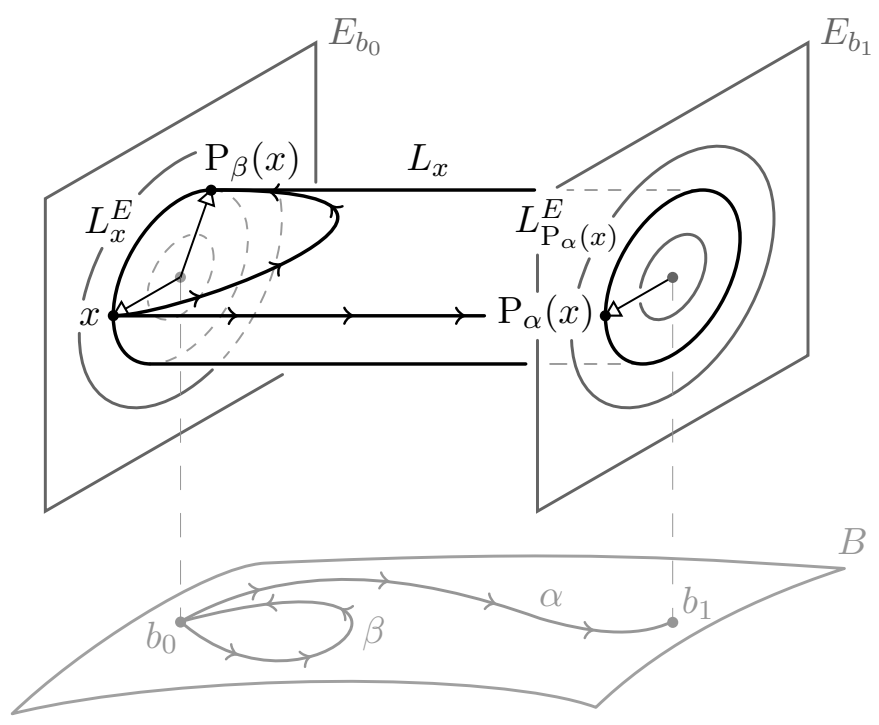

Figura: Esquema da folheação modelo $\mathcal{F}(\nabla)$.

Teorema C. Sejam $(M, g)$ uma variedade Riemanniana, $\mathcal{F}$ uma FRS e $L \in \mathcal{F}$ uma folha fechada. Então, existe uma vizinhança tubular geométrica em torno de $L$ e $\nabla$ uma conexão linear métrica em $\nu L$, tais que $\left.\mathcal{F}\right|_{W}, \mathcal{F}^{\ell}$ e $\mathcal{F}^{\text {lc }}$ são folheadamente difeomorfas a $\mathcal{F}(\nabla), \mathcal{F}(\nabla)^{\ell}$ e $\mathcal{F}(\nabla)^{\text {lc }}$, em uma vizinhança da seção nula de $\nu L$, respectivamente.

A introdução será finalizada com uma sucinta descrição dos capítulos que compõem este texto.

Os Capítulos 1 e 2 consistem em uma breve e rudimentar exposição da teoria de FRS, fortemente baseada em [23], [30], [8], [7], [1] e [4]. Serão estabelecidas nestes capítulos, praticamente, 
xiv

todas as notações, definições, jargões e resultados a respeito da teoria de FRS. Além disto, a idéia central é reunir os resultados relevantes a este texto dispersos nas várias referências citadas, dando provas dos mesmos quando for necessário.

O Capítulo 3 consiste em uma breve e rudimentar exposição sobre grupóides de Lie. Diferentemente do que será desenvolvido nos capítulos 1 e 2, neste capítulo serão apresentados apenas os conceitos e resultados estritamente necessários para a compreensão do texto. Cita-se, como principais referências utilizadas para a elaboração deste capítulo, [22], [11], [19] e [12].

O Capítulo 4 contém os principais resultados deste texto (Teoremas A, B e C já apresentados). Neste capítulo, será apresentada a construção, semi-localmente, de conexões parciais tangentes à folheação, a partir da escolha de distribuições tangentes à folheação, que serão cruciais na prova do Teorema A. Ainda, serão apresentadas distribuições intrínsecas da folheação que, entre outras coisas, irão descrever o fecho da folheação e serão cruciais na prova do Teorema B. 


\section{Capítulo 1.}

\section{Folheações Singulares}

O capítulo inicia-se com a Seção 1.1, onde alguns conceitos acerca de folheações singulares serão apresentados (e.g. funções e campos folheados, subvariedades saturadas, subfolheações, seções transversais). Além disto, nesta seção serão explorados alguns exemplos, dentre os quais destacam-se as folheações homogêneas, as folheações simples e as folheações de holonomia. Já na Seção 1.2 será apresentado o Teorema de Stefan-Sussmann e sua relação visceral com a idéia por trás da definição de folheações.

A idéia neste capítulo é apresentar tudo o quanto for possível e necessário ao texto sobre folheações singulares, sem o uso da estrutura métrica que será explorada no Capítulo 2.

\subsection{Folheações e aplicações folheadas}

Seja $M$ uma variedade diferenciável conexa. Uma partição $\mathcal{F}$ de $M$, por subvariedades imersas (sem auto-intersecções) e conexas, será dita uma folheação singular de $M$ se e somente se dado $L \in \mathcal{F}$ e $(p, v) \in \mathrm{T} L$, existe $X \in \mathfrak{X}(M)$, tal que $\left.X\right|_{p}=v$ e $\left.X\right|_{\tilde{L}} \in \mathfrak{X}(\tilde{L})$, para todo $\tilde{L} \in \mathcal{F}$. Comumente, o par $(M, \mathcal{F})$ será dito uma variedade (singularmente) folheada. Neste caso, cada elemento $L \in \mathcal{F}$ será dito uma folha da folheação $\mathcal{F}$ e, para cada $p \in M$, a única folha que contém $p$ será dita a folha de $\mathcal{F}$ passando por $p$ e será denotada por $L_{p}$.

A condição da existência de campos tangentes às folhas é, na realidade, uma condição local (i.e. basta que dado $L \in \mathcal{F}$ e $(p, v) \in \mathrm{T} L$, exista $U \subset M$ um aberto em torno de $p$ e $X \in \mathfrak{X}(U)$, tais que $\left.X\right|_{U \cap \tilde{L}} \in \mathfrak{X}(U \cap \tilde{L})$, para todo $\left.\tilde{L} \in \mathcal{F}\right)$.
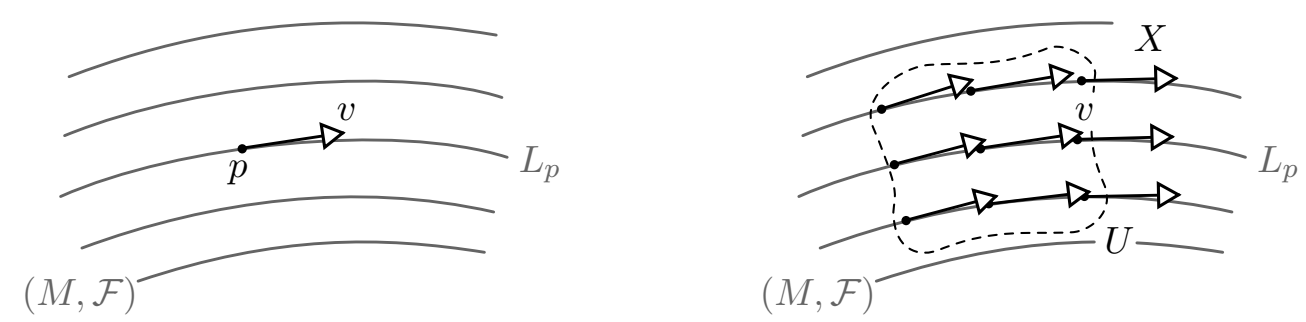

Figura 1.1: Ilustração da condição de existência de campos tangentes às folhas.

Equivalentemente, partição $\mathcal{F}$ de $M$, por subvariedades imersas e conexas é uma folheação singular se e somente se existe $\left\{X_{i}\right\}_{i \in I}$ um conjunto de campos de vetores locais (i.e. definidos 
em abertos de $M)$, tal que $\mathrm{T}_{p} L=\operatorname{span}\left(\left\{\left.X_{i}\right|_{p}\right\}_{i \in I}\right)$, para todo $p \in L$ e $L \in \mathcal{F}$.

Dada uma folheação singular $\mathcal{F}$ a dimensão da folheação será definida por $\operatorname{dim}(\mathcal{F}):=$ $\operatorname{máx}\left\{\operatorname{dim}(L) \in \mathbb{Z}_{+}^{*}: L \in \mathcal{F}\right\}$ e uma folha $L \in \mathcal{F}$ será dita regular se e somente se $\operatorname{dim}(L)=$ $\operatorname{dim}(\mathcal{F})$ e, caso contrário, será dita singular.

Quando todas as folhas de $\mathcal{F}$ forem regulares a folheação singular $\mathcal{F}$ será dita uma folheação regular ou, simplesmente, uma folheação. Cabe ressaltar que este conceito de folheação (regular) é equivalente ao conceito "clássico" de folheação regular dado por atlas folheados, que pode ser encontrado em [10], [23] e [22], por exemplo. A prova desta equivalência pode ser encontrada em [15], por exemplo.

Observação) (Semi-continuidade inferior) Dado $p \in M$, sempre existe $U \subset M$ uma vizinhança de $p$, tal que $\operatorname{dim}\left(L_{q}\right) \geqslant \operatorname{dim}\left(L_{p}\right)$, para todo $q \in U$. De fato, dado $\left\{v_{1}, \cdots, v_{n}\right\}$ uma base de $T_{p} L_{p}$, uma vez que $\mathcal{F}$ é uma folheação, existem $U \subset M$ uma vizinhança aberta de $p$ e $X_{1}, \cdots, X_{n} \in \mathfrak{X}(\mathcal{F})$, tais que $\left.X_{i}\right|_{p}=v_{i}$, para cada $i \in\{1, \cdots, n\}$, e $\left\{\left.X_{1}\right|_{q}, \cdots,\left.X_{n}\right|_{q}\right\}$ é linearmente independente, ou seja, $\operatorname{dim}\left(L_{q}\right) \geqslant \operatorname{dim}\left(L_{p}\right)$, para todo $q \in U$.

Em outras palavras, a função $\operatorname{dim}_{\mathcal{F}}: M \longrightarrow \mathbb{N}$ dada por $\operatorname{dim}_{\mathcal{F}}(p)=\operatorname{dim}\left(L_{p}\right)$ é semi-contínua inferiormente, ou seja, para cada $p \in M$ e $k \in \mathbb{N}$, tais que $\operatorname{dim}_{\mathcal{F}}(p)>k$, existe $U \subset M$ uma vizinhança de $p$, tal que $\operatorname{dim}_{\mathcal{F}}(q)>k$, para todo $q \in U$.

Dadas $(M, \mathcal{F})$ e $\left(N, \mathcal{F}_{N}\right)$ variedades folheadas, uma função $f: M \longrightarrow N$ é dita folheada se e somente se $f$ for diferenciável e $f\left(L_{p}\right) \subset L_{f(p)}$, para todo $p \in M$. Ainda, $f$ será dita um

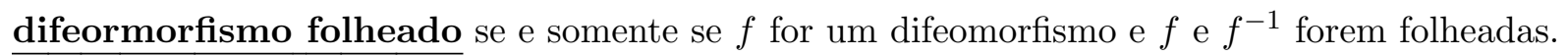
Se $\left(Q, \mathcal{F}_{Q}\right)$ é uma variedade folheada e $f: M \longrightarrow N$ e $g: N \longrightarrow Q$ são funções folheadas, então $f \circ g$ é folheado, de modo que o conjunto

$$
\operatorname{Diff}_{\mathcal{F}}(M):=\left\{f \in \operatorname{Diff}(M): f \text { e } f^{-1} \text { são folheados }\right\}
$$

é um subgrupo do grupo dos difeomorfismo de $M$.

Observação) Um difeomorfismo $f$ é folheado se e somente se $f\left(L_{p}\right)=L_{f(p)}$, para todo $p \in M$. De fato, se $f \in \operatorname{Diff}_{\mathcal{F}}(M)$, então $f^{-1}\left(L_{f(p)}\right) \subset L_{p}$, ou seja, $L_{f(p)} \subset f\left(L_{p}\right)$ e, portanto, como $f$ é folheado, segue que $f\left(L_{p}\right)=L_{f(p)}$, para todo $p \in M$; se, em contrapartida, $f\left(L_{p}\right)=L_{f(p)}$, para todo $p \in M$, então $f$ é automaticamente folheada e, como $f \in \operatorname{Diff}(M)$, dado $q \in M$, sempre existe $p \in M$, tal que $q=f(p)$ e $f^{-1}\left(L_{q}\right)=f^{-1}\left(L_{f(p)}\right)=L_{p}=L_{f^{-1}(q)}$.

Dada $(M, \mathcal{F})$ uma variedade folheada, diz-se que uma função $f: M \longrightarrow M$ fixa a folheação $\mathcal{F}$ se e somente se $f(L) \subset L$, para todo $L \in \mathcal{F}$. Ainda, diz-se que um difeomorfismo $f \in \operatorname{Diff}(M)$ fixa a folheação $\mathcal{F}$ se e somente se $f$ e $f^{-1}$ fixam a folheação $\mathcal{F}$. É imediato desta definição que, dados $f, g \in \operatorname{Diff}(M)$ que fixam $\mathcal{F}$, tem-se que $f \circ g$ fixa $\mathcal{F}$ de modo que

$$
\operatorname{Diff}(\mathcal{F}):=\left\{f \in \operatorname{Diff}(M): f \text { e } f^{-1} \text { fixam } \mathcal{F}\right\}
$$

é um subgrupo e valem as seguintes inclusões $\operatorname{Diff}(\mathcal{F}) \subset \operatorname{Diff}_{\mathcal{F}}(M) \subset \operatorname{Diff}(M)$. 


\subsubsection{Construções básicas e exemplos de folheações singulares}

Serão apresentados, nesta seção, alguns exemplos de folheações singulares.

Alguns deles são meramente protocolares e ilustrativos (e.g. exemplos triviais, produto de folheações, "pullback" e "pushforward" de folheações) e não terão real significância ao longo do texto a não ser pelo estabelecimento de notações e jargões.

Outros exemplos (e.g. órbitas de ações suaves, fibras de uma submersão e folheação de holonomia) estarão presentes em muitas demonstrações e no desenvolvimento do texto. Parte do que ocorre nestes exemplos ilustra bem algumas das idéias e proporcionam uma boa intuição acerca da topologia e da geometria local das folheações singulares. Destaca-se, de antemão, que a folheação de holonomia (desenvolvida no final desta seção) sob certas circunstâncias representa a dinâmica semi-local do que mais a frente será definido por folheação linearizada.

\section{Exemplos triviais - folheações de dimensão máxima e mínima}

Seja $M$ uma variedade diferenciável conexa.

A partição de $M$ dada pelos seus pontos (subvariedades de dimensão 0) é uma folheação (regular) de $M$ a qual será, comumente, denotada por \{pts.\}. Observe que esta é a única folheação de $M$ com dimensão 0 .

A partição de $M$ dada pelo próprio $M$ é uma folheação (regular) de $M$ a qual será, comumente, denotada por $M$, quando não houver o risco de confusão. Observe que esta é a única folheação de $M$ com dimensão igual a dimensão de $M$.

\section{Produto cartesiano de folheações singulares}

$\operatorname{Sejam}\left(M, \mathcal{F}_{M}\right)$ e $\left(N, \mathcal{F}_{N}\right)$ variedades (singularmente) folheadas. Então

$$
\mathcal{F}_{M} \times \mathcal{F}_{N}:=\left\{L^{M} \times L^{N} \subset M \times N: L^{M} \in \mathcal{F}_{M} \text { e } L^{N} \in \mathcal{F}_{N}\right\}
$$

é uma folheação singular de $M \times N$, que será dita a folheação produto de $\mathcal{F}_{M}$ por $\mathcal{F}_{N}$. De fato, dados $L_{p}^{M} \times L_{q}^{N} \in \mathcal{F}_{M} \times \mathcal{F}_{N}$ e $(u, v) \in \mathrm{T}_{(p, q)} \overline{\left(L_{p}^{M} \times L_{q}^{N}\right) \approx \mathrm{T}_{p} L_{p}^{M} \oplus \mathrm{T}_{q} L_{q}^{N} \text {, existem }}$ $X \in \mathfrak{X}\left(\mathcal{F}_{M}\right)$ e $Y \in \mathfrak{X}\left(\mathcal{F}_{N}\right)$, tais que $\left.X\right|_{p}=u$ e $\left.Y\right|_{q}=v$ e, portanto, $Z=(X, Y) \in \mathfrak{X}\left(\mathcal{F}_{M} \times \mathcal{F}_{N}\right)$ é tal que $\left.Z\right|_{(p, q)}=(u, v)$.

\section{"Pullback" e "Pushforward" de folheações singulares}

Sejam $M$ e $N$ variedades diferenciáveis, $\mathcal{F}_{N}$ uma folheação singular de $N$ e $f: M \longrightarrow N$ um difeomorfismo local. Então a partição $f^{*} \mathcal{F}$ dada pelas componentes conexas de $f^{-1}(L)$, com $L \in \mathcal{F}_{N}$, é uma folheação singular de $M$ denominada o "pullback" da folheação $\mathcal{F}_{N}$ por $f$. De fato, é claro que $f^{*} \mathcal{F}$ é uma partição de $M$ e, devido a transversalidade de $f$ com $L$ (pois $f$ é um difeomorfismo), para cada $L \in \mathcal{F}$, esta é uma partição por subvariedades imersas. Ainda, dado $v \in \mathrm{T}_{p} M$, existe $Y \in \mathfrak{X}\left(\mathcal{F}_{N}\right)$, tal que $\left.Y\right|_{f(p)}=\left.\mathrm{d} f\right|_{p}(v)$ e, consequentemente, o campo $f^{*} Y \in \mathfrak{X}(M)$ é tangente a $f^{-1}(L)$, para cada $L \in \mathcal{F}$, com $\left.f^{*} Y\right|_{p}=v$. 
Sejam $M$ e $N$ variedades diferenciáveis, $\mathcal{F}_{M}$ uma folheação singular de $M$ e $f: M \longrightarrow N$ um difeomorfismo. Então

$$
f_{*} \mathcal{F}:=\left\{f(L): L \in \mathcal{F}_{M}\right\}
$$

é uma folheação singular de $N$ denominada o "pushforward" da folheação $\mathcal{F}_{M}$ por $f$. De fato, a sobrejetividade de $f$ garante que $f_{*} \mathcal{F}$ é uma partição de $N$ e o fato de $f$ ser um difeomorfismo garante que esta partição é constituída por subvariedades imersas. Ainda, dado $w \in \mathrm{T}_{q} N$, existe $X \in \mathfrak{X}\left(\mathcal{F}_{M}\right)$, tal que $\left.X\right|_{q}=\left.\mathrm{d} f^{-1}\right|_{q}(w)$ e, consequentemente, o campo $f_{*} X \in \mathfrak{X}(N)$ é tangente a $f(L)$, para cada $L \in \mathcal{F},\left.\operatorname{com} f_{*} X\right|_{q}=w$.

\section{Fibras de uma submersão (folheação simples)}

Sejam $M$ e $B$ variedades diferenciáveis e $\pi: M \longrightarrow B$ uma submersão. Considere $\mathcal{F}^{\pi}$ a partição dada pelas componentes conexas das fibras de $\pi$, ou seja, das componentes conexas de $\pi^{-1}(b)$, com $b \in \operatorname{Im}(\pi)$.

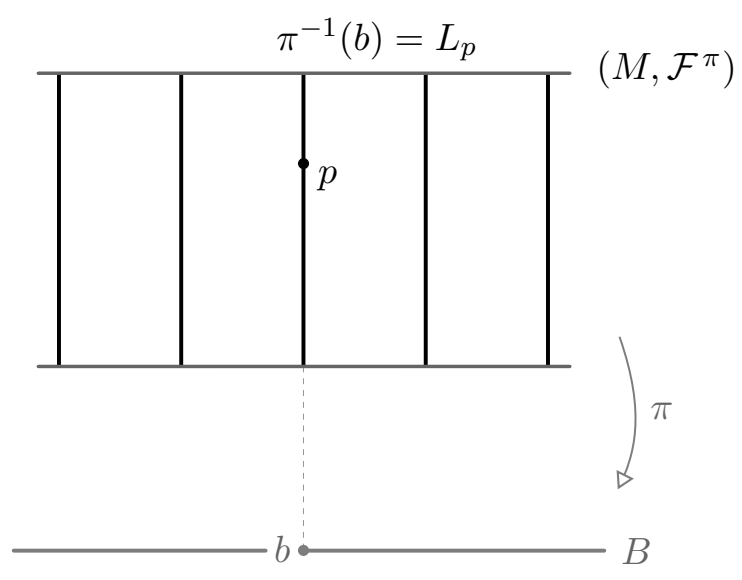

Figura 1.2: Ilustração das fibras da submersão $\pi$.

Proposição) A partição $\mathcal{F}^{\pi}$ é uma folheação (regular) de $M$.

Demonstração. É evidente que a partição $\mathcal{F}^{\pi}$ é dada por subvariedades mergulhadas. Resta provar que dados $p \in M$ e $v \in \mathrm{T}_{p} \pi^{-1}(b)=\operatorname{Ker}\left(\left.\mathrm{d} \pi\right|_{p}\right)$, onde $\pi(p)=b$, existe $X \in \mathfrak{X}_{\text {loc. }}(M)$, tal que $X$ é tangente a $\operatorname{Ker}(\mathrm{d} \pi)$ e $\left.X\right|_{p}=v$.

Considere $\Phi: \mathcal{U} \subset M \longrightarrow \mathbb{R}^{n+k}$ e $\varphi: U \subset B \longrightarrow \mathbb{R}^{n}$ cartas de $M$ e $B$, em torno de $p$ e $b$, respectivamente, tais que $\varphi \circ \pi \circ \Phi^{-1}(x, y)=x$, para todo $(x, y) \in \mathbb{R}^{n} \times \mathbb{R}^{k}$ (forma local das submersões). Observe que $\left.\mathrm{d} \Phi\right|_{p}(v)=\left(0, v_{2}\right)$, uma vez que $v \in \operatorname{Ker}\left(\left.\mathrm{d} \pi\right|_{p}\right)$.

Defina $X_{0} \in \mathfrak{X}(\Phi(\mathcal{U}))$ por $X_{0}(x, y):=\left((x, y),\left(0, v_{2}\right)\right)$ e $X \in \mathfrak{X}(\mathcal{U})$ por $X=\Phi^{*} X_{0}$. Então

- $\left.X\right|_{p}=v$, pois $\left.\Phi^{*} X_{0}\right|_{p}=\left.\mathrm{d} \Phi^{-1}\right|_{\Phi(p)}\left(\left.X_{0}\right|_{\Phi(p)}\right)=v$,

- $\left.X\right|_{q} \in \operatorname{Ker}\left(\left.\mathrm{d} \pi\right|_{q}\right)$, para todo $q \in \mathcal{U}$, pois $\left.\mathrm{d} \pi\right|_{q}\left(\left.X\right|_{q}\right)=\left.\mathrm{d} \pi\right|_{q}\left(\left.\mathrm{~d} \Phi^{-1}\right|_{\Phi(q)}\left(0, v_{2}\right)\right)=0$. 
Assim $\mathcal{F}^{\pi}$ é uma folheação (regular), pois $b \longmapsto \operatorname{dim}\left(\pi^{-1}(b)\right)$ é constante.

A folheação $\mathcal{F}^{\pi}$ será dita a folheação simples dada pela submersão $\pi$.

\section{Órbitas de ações suaves (folheações homogêneas)}

Sejam $M$ uma variedade diferenciável e $G$ um grupo de Lie, com álgebra de Lie g. Uma ação suave (à esquerda) de $G$ sobre $M$ é uma aplicação suave $\mu: G \times M \longrightarrow M$, tal que $\mu(e, p)=p$ e $\mu(g, \mu(h, p))=\mu(g h, p)$, para todo $p \in M$ e $g, h \in G$, onde $e \in G$ é o elemento neutro (ver [3] e [13], por exemplo, para uma abordagem mais completa acerca de ações suaves).

Dada uma ação $\mu$, para cada $p \in M$, será considerado $\mu_{p}: G \longrightarrow M$ o mapa de órbitas dado por $\mu_{p}(g):=\mu(g, p), G_{p}=\{g \in G: \mu(g, p)=p\}$ o grupo de isotropia da ação $\mu$ no ponto $p$ e $G(p)=\{\mu(g, p) \in M: g \in G\}=\operatorname{Im}\left(\mu_{p}\right)$ a órbita da ação $\mu$ passando por $p$. Ainda, para cada $X \in \mathfrak{g}$, será considerado $X^{*} \in \mathfrak{X}(M)$ o campo infinitesimal da ação $\mu$, dado por

$$
\left.X^{*}\right|_{p}:=\left.\frac{\mathrm{d}}{\mathrm{d} t} \mu(\exp (t X), p)\right|_{t=0}=\left.\mathrm{d} \mu_{p}\right|_{e}(X),
$$

que é de fato suave, pois $X^{*}=\mathrm{d} \mu \circ \sigma_{p}$, onde $\sigma_{p}: M \longrightarrow \mathrm{T} G \times \mathrm{T} M$ é dada por $\sigma(p)=\left(X_{e}, 0_{p}\right)$, identificando-se $\mathfrak{g} \approx \mathrm{T}_{e} G$.

Uma ação $\mu$ será dita

- livre se e somente se $G_{p}=\{e\}$, para todo $p \in M$,

- efetiva se e somente se $\cap_{p \in M} G_{p}=\{e\}$,

- própria se e somente se a aplicação $\mathcal{M}: G \times M \longrightarrow G \times M$, dada por $\mathcal{M}(g, p):=$ $(g, \mu(g, p))$ for própria (i.e. $\mathcal{M}^{-1}(K)$ é compacto, para todo $K \subset G \times M$ compacto).

A seguir serão expostos dois resultados centrais na teoria de ações, necessários à prova de que as órbitas de uma ação diferenciável são subvariedades imersas.

Teorema 1.1) (Subgrupo fechado) Seja $G$ um grupo de Lie e $H \subset G$ um subgrupo fechado. Então $H$ é um subgrupo de Lie.

Proposição 1.2) Uma ação $\mu$ é própria se e somente se dados $\left(g_{n}\right)_{n \in \mathbb{N}} \subset G$ e $\left(p_{n}\right)_{n \in \mathbb{N}} \subset M$ convergente, tal que $\left(\mu\left(g_{n}, p_{n}\right)\right)_{n \in \mathbb{N}}$ converge, existe subsequência de $\left(g_{n}\right)_{n \in \mathbb{N}}$ convergente.

Teorema 1.3) Seja $\mu$ uma ação livre e própria. Então $M / G:=\{G(p): p \in M\}$ é uma variedade diferenciável e $\pi: M \longrightarrow M / G$ dada por $\pi(p):=G(p)$ é uma submersão sobrejetora.

A prova, tanto dos teoremas, quanto da proposição, pode ser encontrada em [3], por exemplo. 
A seguir será demonstrado um lema técnico que irá expor os principais "ingredientes" da prova de que as órbitas de uma ação diferenciável são subvariedade imersas (Proposição 1.4).

Lema) Seja $\mu$ uma ação suave. Então, para cada $p \in M$,

a) $G_{p} \subset G$ é um subgrupo de Lie;

b) $\mathrm{T}_{g}\left(g G_{p}\right)=\operatorname{Ker}\left(\left.\mathrm{d} \mu_{p}\right|_{g}\right)$, para todo $g \in G$;

c) a ação de $G_{p}$ sobre $G$, dada pela multiplicação (à esquerda), é livre e própria.

Demonstração. Considere, para cada $g \in G, \mu^{g} \in \operatorname{Diff}(M)$ dado por $\mu^{g}(p):=\mu(g, p)$ e $L_{g}, R_{g}: G \longrightarrow G$ as operações de multiplicação por $g$ à esquerda e à direita, respectivamente.

a) Seja $\left(g_{n}\right)_{n \in \mathbb{N}} \subset G_{p}$, tal que $g_{n} \rightarrow g$. Então $\mu\left(g_{n}, p\right)=p$ e, dado que $\mu$ é suave (em particular contínua), segue que $\mu(g, p)=p$, ou seja, $g \in G_{p}$. Logo $G_{p}$ é sequencialmente fechado e, portanto, fechado.

Assim, pelo Teorema 1.1, tem-se que $G_{p} \subset G$ é um subgrupo de Lie.

b) Será demonstrado, primeiramente, que $\mathrm{T}_{g}\left(g G_{p}\right) \subset \operatorname{Ker}\left(\left.\mathrm{d} \mu_{p}\right|_{g}\right)$.

Dado que $L_{g}\left(G_{p}\right)=g G_{p}$, tem-se que $\left.\mathrm{d} L_{g}\right|_{e}\left(\mathfrak{g}_{p}\right)=\mathrm{T}_{g}\left(g G_{p}\right)$, onde $\mathfrak{g}_{p}$ é a álgebra de Lie de $G_{p}$. Ainda, como $\mu_{p}\left(G_{p}\right)=\{p\}$, tem-se que $\left.\mathrm{d} \mu_{p}\right|_{e}\left(\mathfrak{g}_{p}\right)=0$.

Assim

$$
\left.\mathrm{d} \mu_{p}\right|_{g}\left(\mathrm{~T}_{g}\left(g G_{p}\right)\right)=\left.\mathrm{d}\left(\mu_{p} \circ L_{g}\right)\right|_{e}\left(\mathfrak{g}_{p}\right)=\left.\mathrm{d}\left(\mu^{g} \circ \mu_{p}\right)\right|_{e}\left(\mathfrak{g}_{p}\right)=0
$$

Resta demonstrar que $\mathrm{T}_{g}\left(g G_{p}\right) \supset \operatorname{Ker}\left(\left.\mathrm{d} \mu_{p}\right|_{g}\right)$.

Seja $v \in \operatorname{Ker}\left(\left.\mathrm{d} \mu_{p}\right|_{g}\right)$ e considere $X=\left.\mathrm{d} L_{g^{-1}}\right|_{g}(v) \in \mathfrak{g} \approx \mathrm{T}_{e} G$. Então

$$
\left.\mathrm{d} \mu^{g}\right|_{p}\left(\left.X^{*}\right|_{p}\right)=\left.\mathrm{d} \mu^{g}\right|_{p}\left(\left.\mathrm{~d} \mu_{p}\right|_{e}\left(\left.\mathrm{~d} L_{g-1}\right|_{g}(v)\right)\right)=\left.\mathrm{d}\left(\mu^{g} \circ \mu^{g-1} \circ \mu_{p}\right)\right|_{g}(v)=0,
$$

ou seja, $\left.X^{*}\right|_{p}=0$. Logo, como $t \longmapsto \mu(\exp (t X), p)$ é a curva integral de $X^{*}$ passando por $p$, tem-se que $\mu(\exp (t X), p)=p$, para todo $t \in \mathbb{R}$, ou seja, $t \longmapsto g \exp (t X)$ é uma curva em $g G_{p}$.

Assim

$$
v=\left.\mathrm{d} L_{g}\right|_{e}(X)=\left.\mathrm{d} L_{g}\right|_{e}\left(\left.\frac{\mathrm{d}}{\mathrm{d} t} \exp (t X)\right|_{t=0}\right)=\left.\frac{\mathrm{d}}{\mathrm{d} t} g \exp (t X)\right|_{t=0} \in \mathrm{T}_{g}\left(g G_{p}\right) .
$$

c) É trivial ver que a ação de $G_{p}$ sobre $G$ é livre. Resta provar que esta ação é própria.

Seja $\left(h_{n}\right)_{n \in \mathbb{N}} \subset G_{p}$ e $\left(g_{n}\right)_{n \in \mathbb{N}} \subset G$ convergente, tal que $\left(h_{n} g_{n}\right)_{n \in \mathbb{N}}$ é convergente em $G$. Então, como $h_{n}=h_{n} g_{n} g_{n}^{-1}$, para todo $n \in \mathbb{N}$, tem-se que $\left(h_{n}\right)_{n \in \mathbb{N}}$ é convergente em $G$, uma vez que a multiplicação e a inversão são suaves (em particular, contínuas).

Mas, pelo item (a), $G_{p}$ é fechado, $\log _{0} \lim _{n \rightarrow \infty} h_{n} \in G_{p}$. 
Assim, pela Proposição 1.2, segue que a ação é própria.

Proposição 1.4) Seja $\mu$ uma ação suave. Então $G(p) \subset M$ é uma subvariedade imersa, para todo $p \in M$.

Demonstração. Defina $\tilde{\mu}_{p}: G / G_{p} \longrightarrow M$ por $\tilde{\mu}_{p}\left(g G_{p}\right):=\mu_{p}(g)$. Observe que

- $\tilde{\mu}_{p}$ está bem definida, pois se $g G_{p}=h G_{p}$, então $g^{-1} h \in G_{p}$ e, portanto, $\mu_{p}(g)=\mu_{p}(h)$;

- $\tilde{\mu}_{p}$ é injetora, pois se $\tilde{\mu}_{p}\left(g G_{p}\right)=\tilde{\mu}_{p}\left(h G_{p}\right)$, então $\mu_{p}(g)=\mu_{p}(h)$ e, portanto, $g G_{p}=h G_{p}$.

Uma vez que, pelo item (c) do lema anterior, a ação de $G_{p}$ sobre $G$ é livre e própria segue, pelo Teorema 1.3 , que $G / G_{p}$ é uma variedade e $\pi: G \longrightarrow G / G_{p}$ é uma submersão sobrejetora.

Logo $\tilde{\mu}_{p}$ é suave, pois $\tilde{\mu}_{p} \circ \pi=\mu_{p}$.

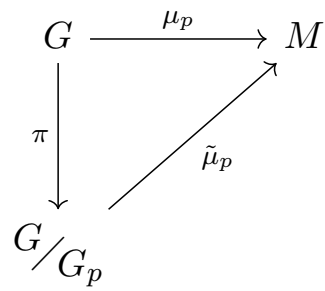

Ainda, pela comutatividade do diagrama, decorre que $\operatorname{Ker}\left(\left.\mathrm{d} \tilde{\mu}_{p}\right|_{g G_{p}}\right)=\left.\mathrm{d} \pi\right|_{g}\left(\operatorname{Ker}\left(\left.\mathrm{d} \mu_{p}\right|_{g}\right)\right)$, para todo $g \in G$. Mas $\operatorname{Ker}\left(\left.\mathrm{d} \mu_{p}\right|_{g}\right) \subset \operatorname{Ker}\left(\left.\mathrm{d} \pi\right|_{g}\right)$, pois $\left.\pi\right|_{g G_{p}}$ é constante e $\operatorname{Ker}\left(\left.\mathrm{d} \mu_{p}\right|_{g}\right)=\mathrm{T}_{g}\left(g G_{p}\right)$, pelo item (b) do lema anterior. Logo $\operatorname{Ker}\left(\left.\mathrm{d} \tilde{\mu}_{p}\right|_{g G_{p}}\right)=\{0\}$, para todo $g \in G$ e, portanto, $\tilde{\mu}_{p}$ é uma imersão injetora.

Assim, como $\pi$ é sobrejetora, tem-se que $G(p)=\operatorname{Im}\left(\mu_{p}\right)=\operatorname{Im}\left(\tilde{\mu}_{p}\right)$, ou seja, $G(p)$ é uma subvariedade imersa de $M$.

A seguir será mostrado que as órbitas da ação $\mu$ formam uma folheação singular de $M$, já ressaltando que ficará claro na demonstração da proposição a seguir que o submódulo $\mathfrak{X}^{G}(M)$ dos campos tangentes às órbitas é dado pelos campos infinitesimais $\left\{X^{*} \in \mathfrak{X}(M): X \in \mathfrak{g}\right\}$.

Proposição 1.5) Seja $\mu$ uma ação suave. Então a partição $\mathcal{F}^{G}=\{G(p)\}_{p \in M}$ é uma folheação singular de $M$.

Demonstração. Já foi visto, na proposição anterior, que $\{G(p)\}_{p \in M}$ é uma partição de $M$ por subvaridades imersas. A seguir será demonstrado que dados $p \in M$ e $v \in \mathrm{T}_{p} G(p)$, existe $X \in \mathfrak{X}(M)$ tangente as órbitas da ação, tal que $\left.X\right|_{p}=v$.

Considere $\pi: G \longrightarrow G / G_{p}$ dada por $\pi(g)=g G_{p}$ e $\tilde{\mu}_{p}: G / G_{p} \longrightarrow M$ dada por $\tilde{\mu}_{p}\left(g G_{p}\right)=$ $\mu_{p}(g)$. Uma vez que $\pi$ é uma submersão (como visto na demonstração da proposição anterior), tem-se que $\left.\mathrm{d} \pi\right|_{e}$ é sobrejetora e, portanto,

$$
\left.\mathrm{d} \mu_{p}\right|_{e}(\mathfrak{g})=\operatorname{Im}\left(\left.\mathrm{d} \tilde{\mu}_{p}\right|_{G_{p}}\right)=\mathrm{T}_{\tilde{\mu}_{p}\left(G_{p}\right)} \tilde{\mu}_{p}\left(G / G_{p}\right)=\mathrm{T}_{p} G(p),
$$


ou seja, existe $X \in \mathfrak{g}$, tal que $\left.X^{*}\right|_{p}=v$.

Mas $X^{*} \in \mathfrak{X}(M)$ é um campo tangente as órbitas da ação, pois $t \longmapsto \mu_{q}(\exp (t X))$ é uma curva suave em $G(q)$, para todo $q \in M$.

Assim $X^{*} \in \mathfrak{X}(M)$ é um campo tangente as órbitas da ação, tal que $\left.X^{*}\right|_{p}=v$.

A folheação $\mathcal{F}^{G}$ será dita a folheação homogênea dada pela ação $\mu$.

\section{Folheação de holonomia}

Sejam $M$ uma variedade diferenciável conexa e $E$ um fibrado vetorial sobre $M$, com projeção $\pi$. Uma distribuição $\mathrm{H} \subset \mathrm{T} E$ será dita horizontal se e somente se $\mathrm{T} E=\operatorname{Ker}(\mathrm{d} \pi) \oplus \mathrm{H}$ e $\left.\mathrm{dm}_{\lambda}\right|_{x}(\mathrm{H})=\left.\mathrm{H}\right|_{\mathrm{m}_{\lambda}(x)}$, para todo $x \in E$ e $\lambda \in \mathbb{R}^{*}$, onde $\mathrm{m}_{\lambda}: E \longrightarrow E$ é o isomorfismo dado por $\mathrm{m}_{\lambda}(x)=\lambda x$ (ver [25] e [33], por exemplo, para uma abordagem mais completa acerca de fibrados, distribuições horizontais e conexões).

Dado H uma distribuição horizontal, considerando-se $C_{\text {par. }}^{\infty}([0,1], M)$ o conjunto das curvas suaves por partes em $M$, para cada $\alpha \in C_{\text {par. }}^{\infty}([0,1], M)$ e $x \in E$, existe uma única curva $\tilde{\alpha}_{x}:[0,1] \subset \mathbb{R} \longrightarrow E$ tangente a $\mathrm{H}$, tal que $\tilde{\alpha}_{x}(0)=x$ e $\pi \circ \tilde{\alpha}_{x}=\alpha$, a saber, ela é dada pela única solução da $\operatorname{EDO}\left(\tilde{\alpha}, \tilde{\alpha}^{\prime}\right)=\left(\left.\mathrm{d} \pi\right|_{\mathrm{H}}\right)^{-1}\left(\alpha, \alpha^{\prime}\right)$, com condição inicial $\tilde{\alpha}(0)=x$. Neste caso, o operador $\mathrm{P}_{\alpha}: E_{\alpha(0)} \longrightarrow E_{\alpha(1)}$ dado por $\mathrm{P}_{\alpha}(x):=\tilde{\alpha}_{x}(1)$ é um isomorfismo que será dito o operador de transporte $\mathrm{H}$-paralelo ao longo de $\alpha$.

A seguir será mostrado que a presença de uma distribuição horizontal $\mathrm{H}$ permitirá a construção de trivializações de $E$ com fibra típica dada por uma fibra fixa de $E$, uma vez que nesta situação todas as fibras de $E$ estão conectadas por meio de transportes paralelos.

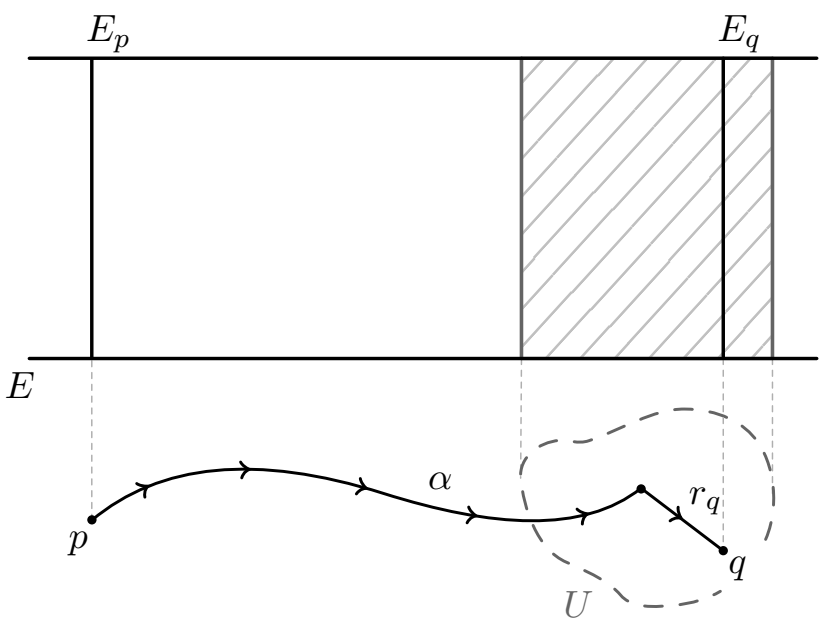

Figura 1.3 : Ilustração da trivialização por tranportes paralelos

Lema) (Trivialização por transportes paralelos) Sejam $p \in M, \alpha \in C_{\text {par. }}^{\infty}([0,1], M)$ partindo de $p$ e $U \subset M$ uma vizinhança coordenada convexa de $\alpha(1)$. Denote por $r_{q}$ a reta contida 
em $U$ que liga $\alpha(1)$ à $q$, para cada $q \in U$. Então $\Phi_{\alpha}: E_{U} \longrightarrow U \times E_{p}$ dada por

$$
\Phi_{\alpha}(y):=\left(\pi(y), \mathrm{P}_{\left(r_{\pi(y)} * \alpha\right)^{-1}}(y)\right)
$$

é uma trivialização de $E$.

Ainda, dado $\tilde{\alpha}$ outra curva partindo de $p$ e $\widetilde{U}$ uma vizinhança coordenada convexa de $\tilde{\alpha}(1)$, se $U \cap \widetilde{U} \neq \emptyset$, então $\Phi_{\alpha}$ e $\Phi_{\tilde{\alpha}}$ são compatíveis, com função de transição $T_{\tilde{\alpha}, \alpha}: U \cap \widetilde{U} \subset M \longrightarrow \mathbb{G L}\left(E_{p}\right)$ dada por

$$
T_{\tilde{\alpha}, \alpha}(q)=\mathrm{P}_{\left(\tilde{r}_{q} * \tilde{\alpha}\right)^{-1}} \circ \mathrm{P}_{\left(r_{q} * \alpha\right)} .
$$

Demonstração. É imediato ver que $\operatorname{pr}_{U} \circ \Phi_{\alpha}=\pi$, onde $\operatorname{pr}_{U}: U \times E_{p} \longrightarrow U$ é a projeção canônica. Ainda, é imediato verificar que $\Phi_{\alpha}$ é bijetora, com inversa dada por $\Phi_{\alpha}^{-1}(q, x)=$ $\mathrm{P}_{\left(r_{q} * \alpha\right)}(x)$, para todo $(q, x) \in U \times E_{p}$. Resta provar que $\Phi_{\alpha}$ e $\Phi_{\alpha}^{-1}$ são suaves.

Defina $F_{\alpha}:[0,1] \times U \times E_{p} \longrightarrow E_{U}$ por $F_{\alpha}(t, q, x):=\mathrm{P}_{r_{q}}^{t}(x)$. Observe que $t \longmapsto F_{\alpha}(t, q, x)$ é o levantamento horizontal de $r_{q}$ com relação a $x$. Dado que o levantamento horizontal de curvas é solução de uma E.D.O. e as curvas $r_{q}$ variam suavemente com relação a $q$, segue que $t \longmapsto F_{\alpha}(t, q, x)$ varia suavemente com relação a $q$ e a $x$ (valor incial da EDO) e, portanto, $F_{\alpha}$ é suave.

Logo, como $\Phi_{\alpha}^{-1}(q, x)=F_{\alpha}(1, q, x)$, segue que $\Phi_{\alpha}^{-1}$ é suave. Analogamente, mostra-se que $\Phi_{\alpha}$ é suave.

Ainda, $\left(\Phi_{\tilde{\alpha}} \circ \Phi_{\alpha}^{-1}\right)(q, x)=\left(q, \mathrm{P}_{\left(\tilde{r}_{q} * \tilde{\alpha}\right)^{-1}}\left(\mathrm{P}_{\left(r_{q} * \alpha\right)}(x)\right)\right)$, para todo $(q, x) \in U \cap \widetilde{U} \times E_{p}$ e, portanto,

$$
T_{\tilde{\alpha}, \alpha}(q)=\mathrm{P}_{\left(\tilde{r}_{q} * \tilde{\alpha}\right)^{-1}} \circ \mathrm{P}_{\left(r_{q} * \alpha\right)} .
$$

Dados $p \in M, \alpha \in C_{\text {par. }}^{\infty}([0,1], M)$ partindo de $p$ e $U \subset M$ uma vizinhança coordenada convexa de $\alpha(1)$, uma trivialização $\Phi_{\alpha}$, como a apresentada no lema anterior, será dita uma trivialização por transportes paralelos.

Além de conectar as fibras do fibrado vetorial $E$ e consequentemente produzir trivializações de $E$, os transportes $\mathrm{H}$-paralelos também permitem particionar $E$, como será visto a seguir.

Dado H uma distribuição horizontal, para cada $x, y \in E$, será dito que $x$ está relacionado com $y$ se, e somente se, existe $\alpha \in C_{\text {par. }}^{\infty}([0,1], M)$, tal que $\mathrm{P}_{\alpha}(x)=y$. A relação, assim definida, é uma relação de equivalência sobre $E$, cuja classe de equivalência correspondente a um elemento $x \in E$ será denotada por $L_{x}$. A partição de $E$ dada por essas classes de equivalência será denotada por $\mathcal{F}^{\mathrm{H}}$ (ver ilustração desta partição na Figura 1.4).

A partir das trivializações por transportes paralelos serão construídas, na Proposição 1.6, trivializações para cada elemento da partição $\mathcal{F}^{\mathrm{H}}$, de modo a torná-lo um subfibrado (não necessariamente vetoriais) de $E$ e, para isto será necessário definir o conceito de holonomia em fibrados vetoriais, como será feito, brevemente, a seguir. 
Dado $p \in M$, será denotado por $\operatorname{Hol}_{p}$ o grupo de holonomia de $\mathrm{H}$ em $p$, a saber,

$$
\operatorname{Hol}_{p}:=\left\{\mathrm{P}_{\gamma} \in \mathbb{G} \mathbb{L}\left(E_{p}\right): \gamma \in C_{\text {par. }}^{\infty}([0,1], M), \gamma(0)=p=\gamma(1)\right\}
$$

Um fato bem conhecido acerca dos grupos de holonomia, que será amplamente utilizado neste texto, é que os mesmos são subgrupos de Lie do grupo linear geral das fibras (Teorema de Ambrose-Singer, cujo enunciado preciso e a prova podem ser encontrados em XXX e XXX, por exemplo)

Proposição 1.6) Seja $H$ uma distribuição horizontal. Então $L_{x} \subset E$ é um subfibrado, com fibra típica $\operatorname{Hol}_{p}(x) \subset E_{p}$, para todo $x \in E$, com $\pi(x)=p$. Em particular, $L_{x} \subset E$ é uma subvariedade imersa.
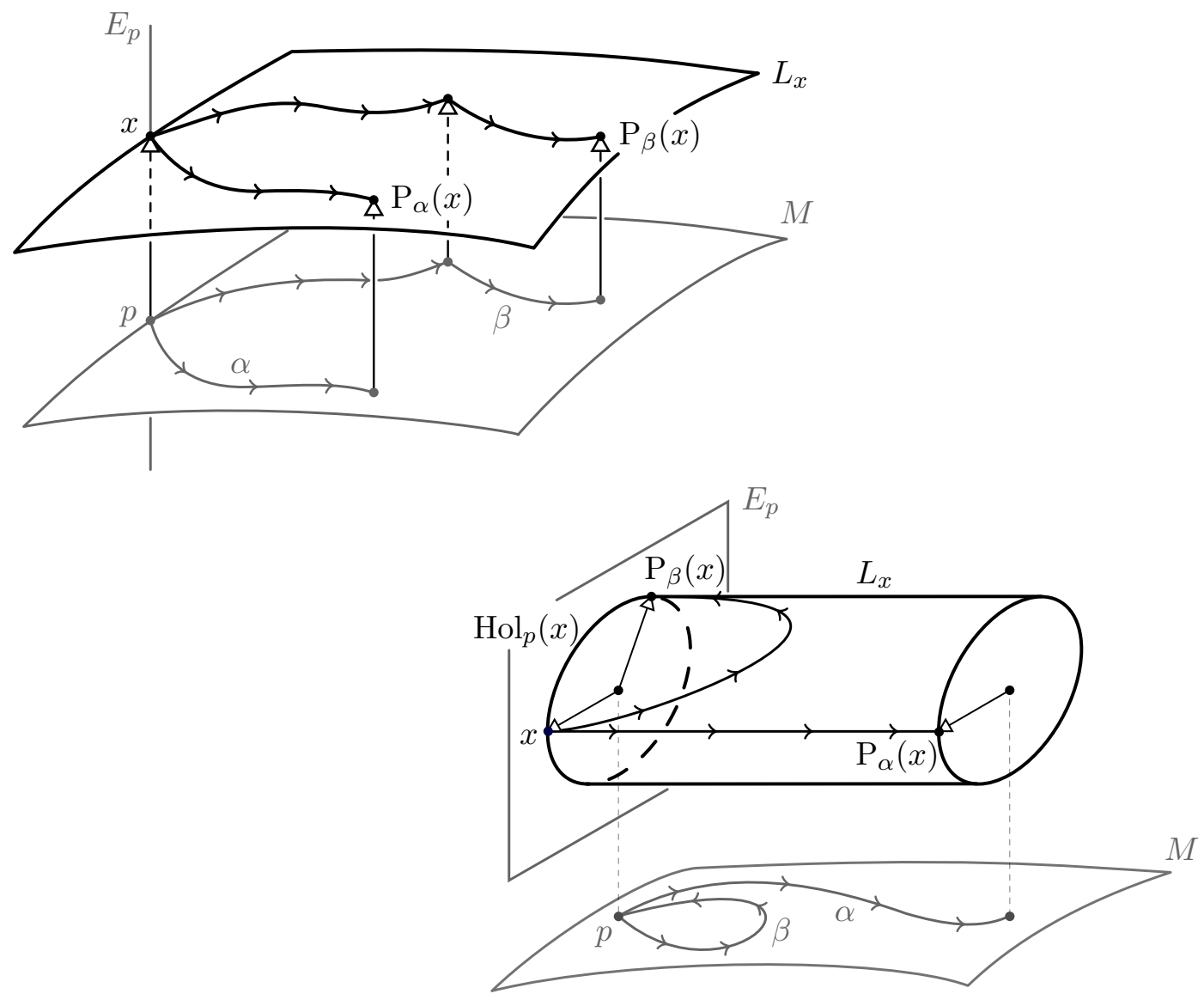

Figura 1.4: Dois esquemas possíveis para ilustrar a partição $\mathcal{F}^{\mathrm{H}}$.

Demonstração. Observe, primeiramente, que $\left.\pi\right|_{L_{x}}: L_{x} \longrightarrow M$ é sobrejetora, pois dado $q \in M$ existe $\alpha \in C_{\text {par. }}^{\infty}([0,1], M)$ ligando $p$ a $q$, de modo que $\pi\left(\mathrm{P}_{\alpha}(\tilde{x})\right)=q$, para qualquer $\tilde{x} \in E_{p}$. Ainda, cabe ressaltar que $\left.\pi\right|_{L_{x}} ^{-1}(U)=L_{x} \cap E_{U}$, para qualquer aberto $U \subset M$.

Dado $\Phi_{\alpha}: E_{U} \longrightarrow U \times E_{p}$ uma trivialização de $E$ por transportes paralelos, tem-se que $\Phi_{\alpha}\left(L_{x} \cap E_{U}\right)=U \times \operatorname{Hol}_{p}(x)$, de modo que a aplicação $\Phi_{\alpha}^{L_{x}}: L_{x} \cap E_{U} \longrightarrow U \times \operatorname{Hol}_{p}(x)$ dada por $\Phi_{\alpha}^{L_{x}}(y)=\Phi_{\alpha}(y)$ fica bem definida. De fato 
- para cada $y \in L_{x} \cap E_{U}$, com $\pi(y)=q$, existe $\beta \in C_{\text {par. }}^{\infty}([0,1], M)$, tal que $y=\mathrm{P}_{\beta}(x)$, de modo que $\Phi_{\alpha}(y)=\left(q, \mathrm{P}_{\left(r_{q} * \alpha\right)^{-1} * \beta}(x)\right) \in U \times \operatorname{Hol}_{p}(x)$;

- para cada $(q, \tilde{x}) \in U \times \operatorname{Hol}_{p}(x)$, existe $\gamma \in C_{\text {par. }}^{\infty}([0,1], M)$, com $\gamma(0)=p=\gamma(1)$, tal que $\tilde{x}=\mathrm{P}_{\gamma}(x)$, de modo que $\Phi_{\alpha}^{-1}(q, \tilde{x})=\mathrm{P}_{\left(r_{q} * \alpha\right) * \gamma}(x) \in L_{x} \cap E_{U}$.

Ainda, $\Phi_{\alpha}^{L_{x}}$ é uma trivialização de $L_{x}, \operatorname{com}\left(\Phi_{\alpha}^{L_{x}}\right)^{-1}(q, \tilde{x})=\Phi_{\alpha}^{-1}(q, \tilde{x})$, para todo $(q, \tilde{x}) \in$ $U \times \operatorname{Hol}_{p}(x)$. De fato

- $\operatorname{pr}_{U} \circ \Phi_{\alpha}^{L_{x}}=\left.\pi\right|_{L_{x}}$, onde $\operatorname{pr}_{U}: U \times \operatorname{Hol}_{p}(x) \longrightarrow U$ é a projeção canônica;

- $\Phi_{\alpha}^{L_{x}}$ é bijetora, pois $\Phi_{\alpha}$ é injetora e, para cada $\left(q, \mathrm{P}_{\gamma}(x)\right) \in U \times \operatorname{Hol}_{p}(x)$, tem-se que $\mathrm{P}_{\left(r_{q} * \alpha\right) * \gamma}(x) \in L_{x} \cap E_{U}$ e $\Phi_{\alpha}\left(\mathrm{P}_{\left(r_{q} * \alpha\right) * \gamma}(x)\right)=\left(q, \mathrm{P}_{\gamma}(x)\right)$.

- $\left(\Phi_{\alpha}^{L_{x}}\right)^{-1}(q, \tilde{x})=\Phi_{\alpha}^{-1}(q, \tilde{x})$, pois $L_{x} \cap E_{U}=\Phi_{\alpha}^{-1}\left(U \times \operatorname{Hol}_{p}(x)\right)$.

Enfim, dada $\Phi_{\tilde{\alpha}}: E_{\widetilde{U}} \longrightarrow \widetilde{U} \times E_{p}$ uma outra trivialização por transportes paralelos, com $U \cap \widetilde{U} \neq \emptyset$, considere $T_{\tilde{\alpha}, \alpha}: U \cap \widetilde{U} \subset M \longrightarrow \mathbb{G} \mathbb{L}\left(E_{p}\right)$ a função de transição entre $\Phi_{\alpha}$ e $\Phi_{\tilde{\alpha}}$.

Dado que $\Phi_{\tilde{\alpha}}^{L_{x}} \circ\left(\Phi_{\alpha}^{L_{x}}\right)^{-1}=\Phi_{\tilde{\alpha}} \circ \Phi_{\alpha}^{-1}$, em $(U \cap \widetilde{U}) \times \operatorname{Hol}_{p}(x)$, e $T_{\tilde{\alpha}, \alpha}(q)\left(\operatorname{Hol}_{p}(x)\right)=\operatorname{Hol}_{p}(x)$, conclui-se que $\Phi_{\tilde{\alpha}}^{L_{x}}$ e $\Phi_{\alpha}^{L_{x}}$ são compatíveis com função de transição $T_{\tilde{\alpha}, \alpha}^{x}: U \cap \widetilde{U} \subset M \longrightarrow$ $\operatorname{Diff}\left(\operatorname{Hol}_{p}(x)\right)$ dada por $T_{\tilde{\alpha}, \alpha}^{x}(q)=\left.T_{\tilde{\alpha}, \alpha}(q)\right|_{\operatorname{Hol}_{p}(x)}$.

Assim

$$
\left\{\Phi_{\alpha}^{L_{x}}:\left.\pi\right|_{L_{x}} ^{-1}(U) \longrightarrow U \times \operatorname{Hol}_{p}(x): \alpha \in C^{\infty}([0,1], M), \alpha(0)=p, U \text { viz. convexa de } \alpha(1)\right\}
$$

é um atlas de trivializações de $L_{x}$ que torna $L_{x}$ um subfibrado de $E$.

O lema a seguir mostrará algumas propriedades das subvariedades da partição $\mathcal{F}^{\mathrm{H}}$ que, além de elucidar aspectos geométricos dessas subvariedades, serão importantes na demonstração de que $\mathcal{F}^{\mathrm{H}}$ é uma folheação singular.

\section{Lema 1.7)}

a) $L_{0_{p}}$ é a seção nula do fibrado vetorial $E$,

b) $\left.\mathrm{H}\right|_{x} \subset \mathrm{T}_{x} L_{x}$,

c) $\left.\mathrm{H}\right|_{0_{p}}=\mathrm{T}_{0_{p}} L_{0_{p}}$,

d) $L_{x} \cap E_{\pi(x)}=\operatorname{Hol}_{\pi(x)}(x)$,

para todo $x \in E$ e $p \in M$.

\section{Demonstração.}

a) Dado $x \in L_{0_{p}}$, existe $\alpha \in C_{\text {par. }}^{\infty}([0,1], M)$ ligando $p$ à $\pi(x)$ e $x=\mathrm{P}_{\alpha}\left(0_{p}\right)=0_{\pi(x)}$.

Dado $q \in M$, seja $\alpha \in C_{\text {par. }}^{\infty}([0,1], M)$ ligando $q$ à $p$. Então $\mathrm{P}_{\alpha}\left(0_{q}\right)=0_{p}$, ou seja, $0_{q} \in L_{0_{p}}$. 
b) Seja $\left.X \in \mathrm{H}\right|_{x}$. Então, existe $\alpha \in C_{\text {par. }}^{\infty}([0,1], M)$, com $\alpha(0)=\pi(x)$, tal que $X=$ $\left.\frac{\mathrm{d}}{\mathrm{d} t} \mathrm{P}_{\alpha}^{t}(x)\right|_{t=0}$. Mas $t \longmapsto \mathrm{P}_{\alpha}^{t}(x)$ é uma curva suave, em $L_{x}$, passando por $x$ no instante $t=0$.

$\operatorname{Assim} X \in \mathrm{T}_{x} L_{x}$, ou seja, $\left.\mathrm{H}\right|_{x} \subset \mathrm{T}_{x} L_{x}$.

c) Segue, do lema anterior, que $\left.\mathrm{H}\right|_{0_{p}} \subset \mathrm{T}_{0_{p}} L_{0_{p}}$. Mas, pelo item (a), tem-se que

$$
\operatorname{dim}\left(\left.\mathrm{H}\right|_{0_{p}}\right)=\operatorname{dim} M=\operatorname{dim}\left(\mathrm{T}_{0_{p}} L_{0_{p}}\right) .
$$

$\left.\operatorname{Assim} \mathrm{H}\right|_{0_{p}}=\mathrm{T}_{0_{p}} L_{0_{p}}$.

d) Dado $y \in L_{x} \cap E_{\pi(x)}$, existe $\alpha \in C_{\text {par. }}^{\infty}([0,1], M)$, tal que $\alpha(0)=\pi(x)$ e $y=\mathrm{P}_{\alpha}(x)$ e, portanto, $\alpha(1)=\pi\left(\mathrm{P}_{\alpha}(x)\right)=\pi(y)=\pi(x)$, ou seja, $\mathrm{P}_{\alpha} \in \operatorname{Hol}_{\pi(x)}$. Logo $y \in \operatorname{Hol}_{\pi(x)}(x)$.

$\operatorname{Assim} L_{x} \cap E_{\pi(x)} \subset \operatorname{Hol}_{\pi(x)}(x)$.

Dado $y \in \operatorname{Hol}_{\pi(x)}(x)$, existe $\alpha \in C_{\text {par. }}^{\infty}([0,1], M)$, tal que $\alpha(0)=\alpha(1)=\pi(x)$ e $y=\mathrm{P}_{\alpha}(x)$ e, portanto, $\pi(y)=\pi\left(\mathrm{P}_{\alpha}(x)\right)=p$, ou seja, $y \in E_{p}$. Logo $y \in L_{x} \cap E_{p}$.

$\operatorname{Assim} L_{x} \cap E_{\pi(x)} \supset \operatorname{Hol}_{\pi(x)}(x)$.

Segue do último item do lema anterior (ilustrado na Figura 1.4) que, para cada $p \in M$, a fibra $E_{p}$ é homogeneamente folheada pela ação do grupo de holonomia $\mathrm{Hol}_{p}$ e tal folheação será denotada por $\mathcal{F}_{p}$.

A seguir será mostrado que campos tangentes à $\mathcal{F}_{p}$ podem ser extendidos à campos de $E$ tangentes às subvariedades da partição $\mathcal{F}^{\mathrm{H}}$. A existência de tais extensões permitirá a conclusão, na Proposição 1.9, de que a partição $\mathcal{F}^{\mathrm{H}}$ é de fato uma folheação singular.

Lema 1.8) (Extensão local de campos por transportes paralelos) Seja $p \in M$ e $X^{p} \in$ $\mathfrak{X}\left(\mathcal{F}_{p}\right)$. Então existe $X \in \mathfrak{X}(E)$, tal que $\left.X\right|_{E_{p}}=X^{p}$ e $\left.X\right|_{y} \in \mathrm{T}_{y} L_{y}$, para todo $y \in E$.

Demonstração. Considere, em torno de $p$, uma vizinhança convexa $U$ e denote por $r_{q}$ a reta em $U$ ligando $p$ a $q$, para cada $q \in U$.

Defina $\widetilde{X} \in \mathfrak{X}\left(E_{U}\right)$ por

$$
\left.\tilde{X}\right|_{y}:=\left(\mathrm{P}_{r_{q}}\right)_{*} X^{p}
$$

com $q=\pi(y)$. Observe que, como $r_{p}$ é a curva constante em $p$, tem-se que $\left.\widetilde{X}\right|_{E_{p}}=X^{p}$. Ainda, como $\mathrm{P}_{r_{q}}$ fixa as subvariedades da partição $\mathcal{F}^{\mathrm{H}}$ e $X^{p}$ é tangente a estas subvariedades, tem-se que $\tilde{X}$ é tangente as subvariedades da partição $\mathcal{F}^{\mathrm{H}}$.

Considerando-se $\Phi: E_{U} \longrightarrow U \times E_{p}$ uma trivialização por transportes paralelos, a saber, dada por $\Phi(y)=\left(\pi(y), \mathrm{P}_{r_{q}}^{-1}(y)\right)$ e definindo-se $\Phi_{p}:=\operatorname{pr}_{E_{p}} \circ \Phi$, onde $\operatorname{pr}_{E_{p}}: U \times E_{p} \longrightarrow E_{p}$ é a projeção canônica, tem-se que

$$
\left.\widetilde{X}\right|_{y}=\Phi^{-1}\left(\pi(y),\left.X^{p}\right|_{\Phi_{p}^{-1}(y)}\right)
$$


para todo $y \in E_{U}$, o que mostra que $\tilde{X}$ é suave.

Assim, obtém-se o campo $X$ desejado extendendo-se $\widetilde{X}$ por meio de uma função auxiliar em $U$ centrada em $p$ (i.e. uma função $f \in C^{\infty}(M,[0,1])$, tal que $\operatorname{supp}(f) \subset U$ e $\left.f\right|_{\widetilde{U}} \equiv 1$, para algum $\widetilde{U} \subset U$ aberto em torno de $p$ ).

Proposição 1.9) Seja $H \subset T E$ uma distribuição horizontal. Então a partição $\mathcal{F}^{\mathrm{H}}$ é uma folheação de $E$.

Demonstração. Sejam $x \in E, \operatorname{com} \pi(x)=p$ e $v \in \mathrm{T}_{x} L_{x}$. Uma vez que $\mathrm{T}_{x} E=\operatorname{Ker}\left(\left.\mathrm{d} \pi\right|_{x}\right) \oplus$ $\left.\mathrm{H}\right|_{x}$, tem-se que $v=v^{\mathrm{V}}+v^{\mathrm{H}}, \operatorname{com} v^{\mathrm{V}} \in \operatorname{Ker}\left(\left.\mathrm{d} \pi\right|_{x}\right) \approx E_{p}$ e $\left.v^{\mathrm{H}} \in \mathrm{H}\right|_{x}$.

Dado que $\mathrm{H}$ é uma distribuição suave, pelo item (b) do lema 1.7, existe $X^{\mathrm{H}} \in \mathfrak{X}(\mathrm{H})$, tal que $\left.X^{\mathrm{H}}\right|_{x}=v^{\mathrm{H}}$ e $\left.X^{\mathrm{H}}\right|_{y} \in \mathrm{T}_{y} L_{y}$, para todo $y \in E$.

Ainda, pelo Lema 1.7, tem-se que $v^{\mathrm{V}}=v-v^{\mathrm{H}} \in \mathrm{T}_{x} L_{x}$, ou seja, $v^{\mathrm{V}} \in \mathrm{T}_{x}\left(L_{x} \cap E_{p}\right)=$ $\mathrm{T}_{x} \operatorname{Hol}_{p}(x)$. Logo, dado que $\mathcal{F}_{p}=\left\{\operatorname{Hol}_{p}(\tilde{x})\right\}_{\tilde{x} \in E_{p}}$ é uma folheação singular em $E_{p}$, existe $X^{p} \in$ $\mathfrak{X}\left(\mathcal{F}_{p}\right)$, tal que $\left.X^{p}\right|_{x}=v^{\mathrm{V}}$ e, pelo Lema 1.8 , existe $X^{\mathrm{V}} \in \mathfrak{X}(E)$, tal que $\left.X^{\mathrm{V}}\right|_{E_{p}}=X^{p}$ e $\left.X^{\mathrm{V}}\right|_{y} \in$ $\mathrm{T}_{y} L_{y}$, para todo $y \in E$ e, em particular, $\left.X^{\mathrm{V}}\right|_{x}=\left.X^{p}\right|_{x}=v^{\mathrm{V}}$.

Assim, $X:=X^{\mathrm{V}}+X^{\mathrm{H}}$ é um campo de vetores em $E$, tal que $\left.X\right|_{x}=\left.X^{\mathrm{V}}\right|_{x}+\left.X^{\mathrm{H}}\right|_{x}=$ $v^{\mathrm{V}}+v^{\mathrm{H}}=v$ e $\left.X\right|_{y} \in \mathrm{T}_{y} L_{y}$, para todo $y \in E$.

A folheação $\mathcal{F}^{\mathrm{H}}$ será dita a folheação de holonomia da distribuição horizontal $\mathrm{H}$ e as folhas de $\mathcal{F}^{\mathrm{H}}$ serão ditas as folhas de holonomia da distribuição horizontal $\mathrm{H}$.

\subsubsection{Seções transversais locais}

Sejam $(M, \mathcal{F})$ uma variedade folheada. Dado $p \in M$, um "slice" ou uma seção transversal local de $\mathcal{F}$ passando por $p$ será uma subvariedade $S_{p}$ mergulhada, conexa e transversal as folhas de $\mathcal{F}$, tal que $\operatorname{dim} S_{p}=\operatorname{codim} L_{p}$ ou, equivalentemente, tal que $\mathrm{T}_{p} M=\mathrm{T}_{p} S_{p} \oplus \mathrm{T}_{p} L_{p}$. Quando $S_{p} \cap L \neq \emptyset$, para todo $L \in \mathcal{F}$, a seção transversal local será simplesmente dita uma seção transversal.

Quando $\mathcal{F}$ é regular, a transversalidade de $S_{p}$ gera uma decomposição do plano tangente a $M$, ao longo de $S_{p}$, a saber, $\mathrm{T}_{q} M=\mathrm{T}_{q} S_{p} \oplus \mathrm{T}_{q} L_{q}$, para todo $q \in S_{p}$, de modo que esta definição de seção transversal local está de acordo com a definição "clássica" de seção transversal dada para folheações regulares, que pode ser encontrada, por exemplo, em [10], [22] e [23]. Neste caso $S_{p}$ é uma seção transversal local passando por $q$, para todo $q \in S_{p}$, de modo que a especificação do ponto $p$, pelo qual a seção passa, é irrelevante.

Lema) (Existência de seções transversais locais) Seja $p \in M$. Então existe uma seção transversal local de $\mathcal{F}$ passando por $p$.

Demonstração. Seja $\Omega \subset L_{p}$ um aberto mergulhado, em torno de $p$, de modo que existe $W$ uma vizinhança tubular em torno de $\Omega$. Observe que $\mathrm{T}_{p} W_{p} \oplus \mathrm{T}_{p} L_{p}=\mathrm{T}_{p} M$, uma vez que 
$W \longrightarrow \Omega$ é uma fibração.

Considere $X \in \mathfrak{X}(\mathcal{F})^{n}$, tal que $\left\{\left.X_{1}\right|_{p}, \cdots,\left.X_{n}\right|_{p}\right\} \subset \mathrm{T}_{p} L_{p}$ é uma base, e $Y \in \mathfrak{X}_{\text {loc. }}\left(W_{p}\right)^{k}$ um referêncial local de $\mathrm{T} W_{p}$ em torno de $p$.

Então, como $\mathrm{T}_{p} W_{p} \oplus \mathrm{T}_{p} L_{p}=\mathrm{T}_{p} M$, existe $U \subset M$ um aberto em torno de $p$, tal que $\left\{X_{1}, \cdots, X_{n}, Y_{1}, \cdots, Y_{k}\right\}$ é um referêncial de $\mathrm{T} M$ em $U \cap \operatorname{Dom}(Y) \subset W_{p}$.

Assim, $S_{p}:=U \cap \operatorname{Dom}(Y)$ é uma subvariedade mergulhada em torno de $p$, tal que $\mathrm{T}_{q} S_{p}+$ $\mathrm{T}_{q} L_{q}=\mathrm{T}_{q} M$, para todo $q \in S_{p}$.

\section{Exemplos.}

a) (Abertos de seções transversais locais) Dada uma variedade folheada $(M, \mathcal{F})$ e $S_{p}$ uma seção transversal local de $\mathcal{F}$ passando por $p$, qualquer aberto $U \subset S_{p}$ em torno de $p$ também é uma seção transversal local de $\mathcal{F}$ passando por $p$.

b) (Folheações triviais) Dada uma variedade diferenciável $M$, qualquer aberto de $M$ é uma seção transversal local da folheação de $M$ dada pelos seus pontos. Já no caso da folheação de $M$ dada pelo próprio $M$, os pontos são as seções transversais locais.

c) Considere $\mathbb{R}^{3}$ folheado por cilindros centrados no "eixo Z", com o "eixo Z" sendo a folha singular. Então o "plano XY" é uma seção transversal a folheação, passando pela "origem", bem como qualquer parabolóide em torno do "eixo Z", centrado na origem.

d) (Folheação simples) Dada uma submersão $\pi: \bar{M} \longrightarrow M$, considere $\mathcal{F}^{\pi}$ a folheação dada pelas fibras de $\pi$ (ver definição na seção 1.1.1). Então, a imagem de qualquer seção local de $\pi$ é uma seção transversal local de $\mathcal{F}^{\pi}$.

e) (Folheação de holonomia) Dada uma distribuição horizontal H em um fibrado vetorial $E \stackrel{\pi}{\longrightarrow} M$, considere $\mathcal{F}^{\mathrm{H}}$ a folheação de holonomia da distribuição $\mathrm{H}$ (ver definição na seção 1.1.1). Então, para cada $p \in M$, a fibra $E_{p}$ é uma seção transversal de $\mathcal{F}^{\mathrm{H}}$ passando por $0_{p}$.

\subsubsection{Subfolheações e subvariedades folheadas}

Seja $M$ uma variedade diferenciável. Dadas $\mathcal{F}$ e $\widetilde{\mathcal{F}}$ folheações singulares de $M$, será dito que $\mathcal{F}$ é uma subfolheação (singular) de $\widetilde{\mathcal{F}}$ se e somente se $L_{p} \subset \tilde{L}_{p}$, para todo $p \in M$, onde $L_{p}$ e $\tilde{L}_{p}$ são as folhas de $\mathcal{F}$ e $\widetilde{\mathcal{F}}$ passando por $p$, respectivamente. Observe que isto define uma relação de ordem parcial no espaço de todas as folheações singulares de $M$, que será denotada por <.

Uma subvariedade folheada de uma variedade folheada $(M, \mathcal{F})$ é uma variedade folheada $\left(N, \mathcal{F}_{N}\right)$, tal que $N \subset M$ é uma subvariedade e $\mathcal{F}_{N}$ é uma subfolheação de $\mathcal{F}$, ou seja, a inclusão $i: N \longrightarrow M$ é folheada, de modo que $\mathcal{F}<\widetilde{\mathcal{F}}$ se e somente se $(M, \mathcal{F})$ é uma subvariedade folheada de $(M, \widetilde{\mathcal{F}})$.

Dada $N \subset M$ uma subavariedade de uma variedade folheada $(M, \mathcal{F})$ será denotada por $\left.\mathcal{F}\right|_{N}$ a partição de $N$ dada pelas componentes conexas da interseção de $N$ com as folhas de $\mathcal{F}$. 
É natural questionar-se quando a partição $\left.\mathcal{F}\right|_{N}$ será uma folheação singular de $N$, de modo que $\left(N,\left.\mathcal{F}\right|_{N}\right)$ torne-se uma subvariedade folheada de $(M, \mathcal{F})$. Um primeiro entrave para que isto ocorra é o fato de que a intersecção de $N$ com as folhas de $\mathcal{F}$ nem sempre é uma subvariedade de $N$. Além disso a condição de que cada vetor tangente a essa intersecção se extenda a um campo tangente aos outros elementos da partição, pode não ser satisfeita.

Existem duas situações triviais para as quais $\left.\mathcal{F}\right|_{N}$ é uma folheação singular de $N$. A primeira situação ocorre quando $N$ é um aberto de $M$, caso no qual, naturalmente, as componentes conexas de $N \cap L$, com $L \in \mathcal{F}$, formam uma folheação de $N$. A segunda situação ocorre quando $L_{p} \subset N$, para todo $p \in N$ e, neste caso a subvariedade $N$ será dita uma subvariedade $\mathcal{F}$-saturada de $M$.

Uma terceira situação pertinente a este texto, que engloba o caso em que $N$ é um aberto, será apresentada mais a frente na Proposição 1.11. Antes disto, faz-se necessário expor o conceito de vizinhança tubular distinguida, que será útil à demonstração de muitas proposições, além da Proposição 1.11 .

Dado $B$ uma subvariedade $\mathcal{F}$-saturada, tal que $\left.\mathcal{F}\right|_{B}$ é uma folheação (regular), e $\Omega \subset B$ um aberto conexo, uma vizinhança tubular distinguida (VTD), em torno de $\Omega$, é uma vizinhança tubular (parcial) folheada $(W, \hat{\mathcal{F}})$, com projeção $\rho: W \longrightarrow B$, tal que

- $\hat{\mathcal{F}}$ é simples (i.e. dada pelas fibras de uma submersão), $\hat{\mathcal{F}}<\left.\mathcal{F}\right|_{W}$ e $\left.\hat{\mathcal{F}}\right|_{\Omega}=\left.\mathcal{F}\right|_{\Omega}$;

- $\mathrm{T}_{p} \hat{L}_{p} \cap \mathrm{T}_{p} W_{\rho(p)}=\{0\}$, para todo $p \in W$.

Cabe ressaltar que a segunda condição implica que $\rho_{\hat{L}}: \hat{L} \longrightarrow B$ é uma imersão, para toda $\hat{L} \in \hat{\mathcal{F}}$. Ainda, quando $B$ é uma folha de $\mathcal{F}$, a segunda condição é equivalente a exigir que as fibras da $W$ sejam seções transversais locais de $\mathcal{F}$ e $\hat{\mathcal{F}}$ passando pelos pontos de $B$.

Observação) (Relação com o conceito topológico de vizinhança tubular) Ao contrário do que sugere o nome, uma vizinhança tubular distinguida não é uma vizinhança tubular, mas é uma vizinhança tubular parcial.

Relembre (ver [14] e [26], por exemplo) que dado $M$ uma variedade diferenciável e $B \subset M$ uma subvariedade (mergulhada), uma vizinhança tubular parcial (resp. vizinhança tubular) em torno de $B$ é uma tripla $(E, U, f)$ (resp. uma par $(E, f))$, onde

- E é um fibrado vetorial sobre $B$, com projeção $\pi$.

- $\mathcal{W} \subset E$ é uma vizinhança aberta da seção nula de $E$ (i.e. um subfibrado aberto de $E$ ),

- $f: \mathcal{W} \longrightarrow M$ (resp. $f: E \longrightarrow M$ ) é um mergulho, tal que $f(\mathcal{W}) \subset M$ (resp. $f(E) \subset M)$ é aberto e $f \circ s_{0}=I d_{N}$, com $s_{0} \in \Gamma(\mathcal{W})$ (resp. $s_{0} \in \Gamma(E)$ ) a seção nula.

Comumente, o aberto $W=f(\mathcal{W})$ (resp. $W=f(E)$ ) é dito uma vizinhança tubular parcial (resp. vizinhança tubular) em torno de $N$. Neste caso $W$ é uma fibração sobre $N$, por meio da projeção $\rho$ : $W \longrightarrow N$, caracterizada por $\rho(f(x))=\pi(x)$, para todo $x \in \mathcal{W}$, de modo que $f$ torna-se um isomorfismo de fibrados sobre $W$.

É um fato bem conhecido (ver [14] e [26]) que toda subvariedade mergulhada de uma variedade diferenciável possui uma vizinhança tubular parcial (resp. vizinhança tubular) em torno de si.

Dentro deste conceito, as vizinhanças tubulares distinguidas não são vizinhanças tubulares, mas são vizinhanças tubulares parciais. 
Proposição 1.10) (Existência de VTD) Seja $B \subset M$ uma subvariedade saturada conexa, tal que $\left.\mathcal{F}\right|_{B}$ é uma folheação (regular). Então, dados $b \in B$ e $X \in \mathfrak{X}(\mathcal{F})^{n}$, tal que $\left\{\left.X_{1}\right|_{b}, \cdots,\left.X_{n}\right|_{b}\right\} \subset$ $\mathrm{T}_{b} L_{b}$ é uma base, existem $\delta>0$,

- $S_{b}$ uma seção transversal local de $\mathcal{F}$ passando por $b$,

- $W$ uma vizinhança tubular (parcial), em torno de uma vizinhança aberta $\Omega \subset B$ de $b$, tais que $\phi^{X}:(-\delta, \delta)^{n} \times S_{b} \longrightarrow W$ dada por

$$
\phi^{X}(t, p):=\left(\phi_{t_{n}}^{X_{n}} \circ \cdots \circ \phi_{t_{1}}^{X_{1}}\right)(p)
$$

é um difeomorfismo, onde $\phi^{X_{i}}$ é o fluxo de $X_{i}$, para cada $i \in\{1, \cdots, n\}$.

Ainda, $\left(W, \mathcal{F}^{X}\right)$ é uma VTD, considerando-se $\mathcal{F}^{X}=\left\{\phi^{X}\left((-\delta, \delta)^{n} \times\{p\}\right)\right\}_{p \in S_{b}}$.

Demonstração. Uma vez que $\left\{\left.X_{1}\right|_{b}, \cdots,\left.X_{n}\right|_{b}\right\}$ é uma base de $\mathrm{T}_{b} L_{b}$, existe $U \subset M$ uma vizinhança aberta em torno de $p$, tal que $\left\{X_{1}, \cdots, X_{n}\right\}$ é linearmente independente em $U$.

Considere

- $\Omega \subset B \cap U$ uma vizinhança aberta de $b$;

- $S_{b}^{B} \subset \Omega$ uma seção transversal local de $\mathcal{F}_{B}$ passando por $b$;

- $W \subset U$ uma vizinhança tubular (parcial) em torno de $\Omega$ com projeção $\rho: W \longrightarrow \Omega$, tal que $S_{b}=\rho^{-1}\left(S_{b}^{B}\right)$ é uma seção transversal local de $\mathcal{F}$ passando por $b$;

- $\delta>0$, tal que $S_{b} \subset \operatorname{Dom}\left(\phi_{t_{1}}^{X_{1}}\right)$ e $\phi_{t_{i}}^{X_{i}}\left(\phi_{t_{(i-1)}}^{X_{(i-1)}}\left(\cdots \phi_{t_{1}}^{X_{1}}\left(S_{b}\right) \cdots\right)\right) \subset \operatorname{Dom}\left(\phi_{t_{(i+1)}}^{X_{(i+1)}}\right)$, para todo $i \in\{1, \cdots, n-1\}$ e $t \in(-\delta, \delta)^{n}$.

Uma vez que $\phi^{X}$ é dada pela composição de fluxos, segue que $\phi^{X}$ é suave. Ainda, como $\left.\mathrm{d} \phi^{X}\right|_{(0, b)}(\lambda, u)=\left.\sum_{i=1}^{n} \lambda_{i} X_{i}\right|_{b}+u$, para todo $(\lambda, u) \in \mathbb{R}^{n} \oplus \mathrm{T}_{b} S_{b}$, segue que $\left.\mathrm{d} \phi^{X}\right|_{(0, b)}$ é um isomorfismo, pois

- $\left.\mathrm{d} \phi^{X}\right|_{(0, b)}$ é injetor, dado que se $(\lambda, u) \in \operatorname{Ker}\left(\left.\mathrm{d} \phi^{X}\right|_{(0, b)}\right)$, então

$$
u=-\left.\sum_{i=1}^{n} \lambda_{i} X_{i}\right|_{b} \in \mathrm{T}_{b} L_{b} \cap \mathrm{T}_{b} S_{b}=\{0\}
$$

e, como $\left\{\left.X_{i}\right|_{b}\right\}_{i=1, \cdots, n}$ é linearmente independente, tem-se que $\lambda_{i}=0$, para todo $i \in$ $\{1, \cdots, n\}$,

- $\operatorname{dim}\left(\mathbb{R}^{n} \oplus \mathrm{T}_{p} S_{b}\right)=\operatorname{dim}\left(L_{b}\right)+\operatorname{dim}\left(S_{b}\right)=\operatorname{dim}(W)$.

Assim, pelo teorema da função inversa, $\Phi^{X}$ é um difeomorfismo em torno de $(0, b)$ e, portanto, reduzindo-se $\delta$ e $S_{b}$ se necessário, $\phi^{X}$ é um difeomorfismo.

Ainda, defina $\pi^{b}: W \longrightarrow S_{b}$, por $\pi^{b}\left(\phi^{X}(t, p)\right)=p$. Então $\pi^{b}$ é uma submersão, pois

$$
\left.\mathrm{d} \pi^{b}\right|_{p}\left(\left.\mathrm{~d} \phi^{X}\right|_{\left(\phi^{X}\right)^{-1}(p)}(0, u)\right)=u
$$


para todo $p \in W$ e $u \in \mathrm{T}_{p} S_{b}$.

Logo, como

$$
\left(\pi^{b}\right)^{-1}(\{p\})=\phi^{X}\left((-\delta, \delta)^{n} \times\{p\}\right),
$$

para todo $p \in W$, segue que $\mathcal{F}^{X}$ é folheação simples dada pela submersão $\pi^{b}$.

Dado que $X_{1}, \cdots, X_{n}$ são campos tangente a $\mathcal{F}$, tem-se que

$$
\phi^{X}\left((-\delta, \delta)^{n} \times\{p\}\right) \subset L_{p}=L_{\phi^{X}(t, p)},
$$

para todo $(t, p) \in(-\delta, \delta)^{n} \times S_{b}$ e, portanto, $\mathcal{F}^{X}<\left.\mathcal{F}\right|_{W}$. Ainda, como $\left\{X_{1}, \cdots, X_{n}\right\}$ é um frame de $L_{\tilde{b}}$, tem-se que $\phi^{X}\left((-\delta, \delta)^{n} \times\{\tilde{b}\}\right)=L_{\phi^{X}(t, \tilde{b})}$, para todo $\left.(t, \tilde{b}) \in-\delta, \delta\right)^{n} \times\left(S_{b} \cap \Omega\right)$ e, portanto, $\left.\mathcal{F}^{X}\right|_{\Omega}<\left.\mathcal{F}\right|_{\Omega}$.

$\operatorname{Assim}\left(W, \mathcal{F}^{X}\right)$ é uma VTD.

A seção será encerrada com a prova de que a partição $\left.\mathcal{F}\right|_{N}$ é uma folheação singular, quando $N \subset M$ é uma subvariedade transversal às folhas de $\mathcal{F}$. Isto seguirá como consequência deste fato para o caso regular que será exposto a seguir e cuja prova pode ser encontrada em [10], por exemplo.

Proposição) ([10], pág. 31) Sejam $M$ e $N$ variedades diferenciáveis, $\mathcal{F}$ uma folheação (regular) de $M$ e $f: N \longrightarrow M$ um função transversal a $\mathcal{F}$. Dado $q \in N$, considere $f^{*} L_{q}$ a componente conexa de $f^{-1}\left(L_{f(q)}\right)$ passando por $q$. Então $f^{*} \mathcal{F}=\left\{f^{*} L_{q}: q \in N\right\}$ é uma folheação (regular).

Proposição 1.11) Sejam $(M, \mathcal{F})$ uma variedade folheada $N \subset M$ uma subvariedade transversal as folhas de $\mathcal{F}$. Então $\left.\mathcal{F}\right|_{N}$ é uma folheação singular de $N$, tal que $\left(N,\left.\mathcal{F}\right|_{N}\right)$ é uma subvariedade folheada de $(M, \mathcal{F})$.

Demonstração. Segue, da transversalidade de $N \operatorname{com} \mathcal{F}$, que $L_{p}^{N} \subset N$ é uma subvariedade imersa, de modo que $\left.\mathcal{F}\right|_{N}$ é uma partição de $N$ por subvariedades imersas. Resta provar que, dado $v \in \mathrm{T}_{p} L_{p}^{N}=\mathrm{T}_{p} N \cap \mathrm{T}_{p} L_{p}$, existe $X \in \mathfrak{X}(N)$, tal que $\left.X\right|_{p}=v$ e $X$ é tangente a $L^{N}$, para todo $\left.L^{N} \in \mathcal{F}\right|_{N}$.

Considere $\Omega \subset L_{p}$ uma vizinhança aberta conexa de $p$ e $(W, \hat{\mathcal{F}})$ uma VTD em torno de $\Omega$.

Então, reduzindo-se $W$ caso necessário, $U=N \cap W$ é transversal a $\hat{\mathcal{F}}$. De fato, como $\hat{L}_{p}=\Omega$ e $N$ é transversal a $\mathcal{F}$, tem-se que $\mathrm{T}_{p} N+\mathrm{T}_{p} \hat{L}_{p}=\mathrm{T}_{p} M$ e então, dado que $\hat{\mathcal{F}}$ é regular, existe $\widetilde{U} \subset N$ vizinhança de $p$, tal que $\mathrm{T}_{q} N+\mathrm{T}_{q} \hat{L}_{q}=\mathrm{T}_{q} M$, para todo $q \in \widetilde{U}$. Reduzindo-se $W$ para que $U \subset \widetilde{U}$, tem-se o afirmado.

Segue, da Proposição anterior, que $\left.\hat{\mathcal{F}}\right|_{U}$ é uma folheação (regular). Logo, como $v \in \mathrm{T}_{p} N \cap$ $\mathrm{T}_{p} L_{p}=\mathrm{T}_{p}\left(U \cap \hat{L}_{p}\right)$, existe $X \in \mathfrak{X}(U)$ campo vetorial tangente as folhas de $\left.\hat{\mathcal{F}}\right|_{U}$, tal que $\left.X\right|_{p}=v$.

Mas, como $\hat{\mathcal{F}}<\left.\mathcal{F}\right|_{W}$, tem-se que $\hat{L}_{q} \cap U \subset L_{q} \cap U$, para todo $q \in U$, de modo que $X$ é tangente as subvariedades da partição $\left.\mathcal{F}\right|_{U}$.

Assim, extendendo-se $X$ por uma função auxiliar em torno de $p$ e suportada em $U$, obtém-se $X \in \mathfrak{X}(N)$, tal que $\left.X\right|_{p}=v$ e $X$ é tangente as subvariedades da partição $\left.\mathcal{F}\right|_{N}$. 
Observação) ("Pullback" de folheações) Procedendo-se de modo análogo ao da prova da proposição anterior, é possível mostrar que, dada $f: N \longrightarrow M$ transversal as folhas de $\mathcal{F}$, a partição $f^{*} \mathcal{F}$ dada pelas componentes conexas das subvariedades $f^{-1}(L)$, com $L \in \mathcal{F}$, é uma folheação singular.

Resumindo, é possível considerar o "pullback" de folheações singulares por meio de aplicações transversais, o que engloba o conceito de "pullback" por meio de difeomorfismos locais, apresentado na Seção 1.1.1.

\subsubsection{Campos verticais e folheados}

Seja $(M, \mathcal{F})$ uma variedade folheada. Um campo diferenciável $V \in \mathfrak{X}(M)$ será dito vertical ou tangente à folheação $\mathcal{F}$ se e somente se $\left.V\right|_{p} \in \mathrm{T}_{p} L_{p}$, para todo $p \in M$. O conjunto dos campos verticais será denotado por $\mathfrak{X}(\mathcal{F})$, de modo que $\mathfrak{X}(\mathcal{F}) \subset \mathfrak{X}(M)$ é um $C^{\infty}(M)$-submódulo de campos. Mais adiante, será mostrado que $\mathfrak{X}(\mathcal{F}) \subset \mathfrak{X}(M)$ também é uma subálgebra de Lie.

Um campo $X \in \mathfrak{X}(M)$ será dito folheado se e somente se $[X, V] \in \mathfrak{X}(\mathcal{F})$, para todo $V \in \mathfrak{X}(\mathcal{F})$, onde $[\cdot, \cdot]$ é o colchete de Lie dos campos diferenciáveis de $M$. O conjunto dos campos folheados será denotado por $\mathfrak{X}_{\mathcal{F}}(M)$.

Segue, da linearidade do colchete de campos, que $\mathfrak{X}_{\mathcal{F}}(M) \subset \mathfrak{X}(M)$ é um subespaço vetorial. Ainda, pela identidade de Jacobi, tem-se que $[[X, Y], V]=[X,[Y, V]]-[Y,[X, V]]$, para todo $X, Y \in \mathfrak{X}_{\mathcal{F}}(M)$ e $V \in \mathfrak{X}(\mathcal{F})$ e, portanto, $[X, Y] \in \mathfrak{X}_{\mathcal{F}}(M)$, para todo $X, Y \in \mathfrak{X}_{\mathcal{F}}(M)$, de modo que $\mathfrak{X}_{\mathcal{F}}(M) \subset \mathfrak{X}(M)$ é uma subálgebra de Lie.

Entretanto, $\mathfrak{X}_{\mathcal{F}}(M) \subset \mathfrak{X}(M)$ não é necessariamente um $C^{\infty}(M)$-submódulo, pois se $f \in$ $C^{\infty}(M), X \in \mathfrak{X}_{\mathcal{F}}(M)$ e $V \in \mathfrak{X}(\mathcal{F})$, então $[f X, V]=-V(f) X+f[X, V]$ e $V(f) X$ não é necessariamente um campo tangente a folheação.

Lema) Seja $X \in \mathfrak{X}(M)$, com $\phi: D \subset \mathbb{R} \times M \longrightarrow M$ o fluxo de $X$. Se o fluxo de $X$ no instante $t$ é folheado, para todo $t \in \mathbb{R}$, então $X$ é folheado.

Demonstração. Seja $V \in \mathfrak{X}(\mathcal{F})$. Então d $\left.\phi_{-t}\right|_{\phi_{t}(p)}\left(\left.V\right|_{\phi_{t}(p)}\right) \in \mathrm{T}_{p} L_{p}$, para todo $(t, p) \in D$, uma vez que o fluxo de $X$ é folheado.

Assim, como

$$
\left.[X, V]\right|_{p}=\lim _{t \rightarrow 0} \frac{\left.\mathrm{d} \phi_{-t}\right|_{\phi_{t}(p)}\left(\left.V\right|_{\phi_{t}(p)}\right)-\left.V\right|_{p}}{t},
$$

segue que $\left.[X, V]\right|_{p} \in \mathrm{T}_{p} L_{p}$, para todo $p \in M$, ou seja, $[X, V] \in \mathfrak{X}(\mathcal{F})$.

Corolário) $\mathfrak{X}(\mathcal{F}) \subset \mathfrak{X}_{\mathcal{F}}(M)$ é ideal. Em particular, $\mathfrak{X}(\mathcal{F}) \subset \mathfrak{X}(M)$ uma subálgebra de Lie.

Demonstração. Dado $X \in \mathfrak{X}(\mathcal{F})$ segue que as curvas integrais de $X$ estão contidas nas folhas de $\mathcal{F}$ e, portanto, o fluxo de $X$ em um instante $t$ é folheado, para todo $t \in \mathbb{R}$ (i.e. se $\phi: D \subset \mathbb{R} \times M \longrightarrow M$ é o fluxo de $X$, então $\phi_{t}\left(D_{t} \cap L\right) \subset L$, para todo $L \in \mathcal{F}$ e $\left.t \in \mathbb{R}\right)$. Logo, pelo lema anterior, tem-se que $X \in \mathfrak{X}_{\mathcal{F}}(M)$. 
Assim $\mathfrak{X}(\mathcal{F}) \subset \mathfrak{X}_{\mathcal{F}}(M)$ e, como $\mathfrak{X}(\mathcal{F})$ é um subespaço vetorial e $[X, V] \in \mathfrak{X}(\mathcal{F})$, para todo $X \in \mathfrak{X}_{\mathcal{F}}(M)$ e $V \in \mathfrak{X}(\mathcal{F})$, conclui-se que $\mathfrak{X}(\mathcal{F})$ é um ideal $\mathfrak{X}_{\mathcal{F}}(M)$.

\section{2. Órbitas de campos e o teorema de Stefan-Sussmann}

Seja $M$ uma variedade diferenciável, cuja topologia será denotada por $\tau_{M}$. Considere, para cada $U, V \in \tau_{M}$, Diff $(U, V)$ o conjunto dos difeomorfismos entre $U$ e $V$ e $\mathfrak{X}(U)$ o conjunto dos campos diferenciáveis de $M$ definidos em $U$. Ainda, considere

$$
\operatorname{Diffloc.}_{\text {lof }}(M):=\bigcup_{U, V \in \tau_{M}} \operatorname{Diff}(U, V), \quad \mathfrak{X}_{\text {loc. }}(M):=\bigcup_{U \in \tau_{M}} \mathfrak{X}(U),
$$

os conjuntos de difeomorfismos locais e campos vetoriais locais, respectivamente.

Dado um subconjunto $\mathfrak{D} \subset \mathfrak{X}_{\text {loc. }}(M)$, o conjunto dos fluxos dos campos de $\mathfrak{D}$ gera, por meio de composições, um pseudogrupo de difeomorfismo $\Gamma(\mathfrak{D})$, o qual será descrito com detalhes a seguir.

Dado $n \in \mathbb{Z}_{+}^{*}$ e $X=\left(X_{1}, \cdots, X_{n}\right) \in \mathfrak{X}_{\text {loc. }}(M)^{n}$, para cada $t \in \mathbb{R}^{n}$, os conjuntos

$$
\begin{aligned}
V^{k} & := \begin{cases}\phi_{t_{1}}^{X_{1}}\left(D_{t_{1}}^{X_{1}}\right), & \text { se } k=1, \\
\phi_{t_{k}}^{X_{k}}\left(V^{(k-1)} \cap D_{t_{k}}^{X_{k}}\right), & \text { se } k \in\{2, \cdots, n\},\end{cases} \\
U^{k}: & = \begin{cases}D_{t_{1}}^{X_{1}}, & \text { se } k=1, \\
\left(\phi_{t_{(k-1)}}^{X_{(k-1)}} \circ \cdots \circ \phi_{t_{1}}^{X_{1}}\right)^{-1}\left(V^{(k-1)} \cap D_{t_{k}}^{X_{k}}\right), & \text { se } k \in\{2, \cdots, n\},\end{cases}
\end{aligned}
$$

são abertos, onde $D_{t_{i}}^{X_{i}} \subset M$ é o domínio do fluxo de $X_{i}$ no instante $t_{i}$, para cada $i \in\{1, \cdots, n\}$ e, portanto, $D_{t}^{X}:=U^{n}$ é um aberto de $M$. Considere $I^{X}:=\left\{t \in \mathbb{R}^{n}: D_{t}^{X} \neq \emptyset\right\}$ de modo que, para cada $t \in I^{X}$, a aplicação $\phi_{t}^{X}: D_{t}^{X} \longrightarrow \phi_{t}^{X}\left(D_{t}^{X}\right)$ dada por $\phi_{t}^{X}:=\phi_{t_{n}}^{X_{n}} \circ \cdots \circ \phi_{t_{1}}^{X_{1}}$ esteja bem definida e seja um difeomorfismo local, onde $\phi_{t_{i}}^{X_{i}}$ é o fluxo de $X_{i}$ no instante $t_{i}$, para cada $i \in\{1, \cdots, n\}$.

A título de ilustração, quando $n=1$, considerando-se $\phi^{X}: D^{X} \subset \mathbb{R} \times \operatorname{Dom}(X) \longrightarrow \operatorname{Dom}(X)$ o fluxo do campo $X$ e $\pi_{\mathbb{R}}: \mathbb{R} \times M \longrightarrow M$ a projeção canônica, tem-se que $I^{X}=\pi_{\mathbb{R}}\left(D^{X}\right)$ e, para cada $t \in I^{X}$, tem-se que $D_{t}^{X}=\left\{p \in \operatorname{Dom}(X):(t, p) \in D^{X}\right\}$ e $\phi_{t}^{X}(p)=\phi^{X}(t, p)$.

Seguindo a notação estabelecida, o pseudogrupo gerado por $\mathfrak{D}$ será, precisamente, dado por

$$
\begin{aligned}
\Gamma(\mathfrak{D}) & =\left\{\phi_{t}^{X} \in \operatorname{Diff}_{\text {loc. }}(M): X \in \mathfrak{D}^{n}, t \in I^{X} \subset \mathbb{R}^{n}, n \in \mathbb{Z}_{+}^{*}\right\} \\
& =\left\{\phi_{t_{n}}^{X_{n}} \circ \cdots \circ \phi_{t_{1}}^{X_{1}} \in \operatorname{Diffloc.~}_{\text {loc }}(M): n \in \mathbb{Z}_{+}^{*}, X_{i} \in \mathfrak{D}, t_{i} \in I^{X_{i}} \subset \mathbb{R}, \forall i \in\{1, \cdots, n\}\right\} .
\end{aligned}
$$

e a órbita desse pseudogrupo, passando por $p \in M$, é dada por

$$
\mathfrak{D}(p)=\left\{\phi_{t}^{X}(p) \in M: X \in \mathfrak{D}^{n}, t \in I^{X} \subset \mathbb{R}^{n}, n \in \mathbb{Z}_{+}^{*}: p \in D_{t}^{X}\right\}
$$


e será dita a órbita de $\mathfrak{D}$ ou a $\underline{\mathfrak{D} \text {-órbita, }}$, passando por $p$. Intuitivamente, $\mathfrak{D}(p)$ constitui-se de todos os pontos de $M$ que podem ser conectados a $p$ por meio da concatenação de curvas integrais de campos de $\mathfrak{D}$.

Uma distribuição singular (suave) $\Delta$ em $M$ será uma aplicação que a cada $p \in M$ associa um subespaço vetorial $\left.\Delta\right|_{p} \subset \mathrm{T}_{p} M$, para o qual existam $n \in \mathbb{Z}_{+}^{*}$ e $X_{1}, \cdots, X_{n} \in \mathfrak{X}_{\text {loc. }}(M)$, tais que

- $p \in \cap_{k=1}^{n} \operatorname{Dom}\left(X_{k}\right)$ e $\operatorname{span}\left(\left\{\left.X_{1}\right|_{p}, \cdots,\left.X_{n}\right|_{p}\right\}\right)=\left.\Delta\right|_{p}$

- $\left.\left.X_{k}\right|_{q} \in \Delta\right|_{q}$, para todo $k \in\{1, \cdots, n\}$ e $q \in \operatorname{Dom}\left(X_{k}\right)$.

Quando $p \longmapsto \operatorname{dim}\left(\left.\Delta\right|_{p}\right)$ for constante, a distribuição singular $\Delta$ é uma distribuição.

Dado uma distribuição singular $\Delta$, uma subvariedade integral de $\Delta$ será uma subvariedade imersa $N \subset M$, tal que $\mathrm{T}_{q} N=\left.\Delta\right|_{q}$, para todo $q \in N$. Ainda, $N$ será dita maximal se e somente se $N$ for conexa e não existir $\widetilde{N} \subset M$ uma subvariedade integral conexa de $\Delta$, tal que $N \varsubsetneqq \widetilde{N}$.

Dado um pseudogrupo de difeomorfismos $\Gamma \subset \operatorname{Diff}_{\text {loc. }}(M)$, uma distribuição singular $\Delta$ será dita $\Gamma$-invariante se e somente se $\left.\mathrm{d} f\right|_{p}\left(\left.\Delta\right|_{p}\right)=\left.\Delta\right|_{f(p)}$, para todo $p \in \operatorname{Dom}(f)$ e $f \in \Gamma$. Ainda, dado $\mathfrak{D} \subset \mathfrak{X}_{\text {loc. }}(M)$ um subconjunto, $\Delta$ será dita $\mathfrak{D}$-invariante se e somente se for $\Gamma(\mathfrak{D})$ invariante ou, equivalentemente, se e somente se $\left.\mathrm{d} \phi_{t}^{X} \overline{\mid}_{p}\left(\left.\Delta\right|_{p}\right) \subset \Delta\right|_{\phi_{t}^{X}(p)}$, para todo $t \in I^{X} \subset \mathbb{R}$, $p \in D_{t}^{X}$ e $X \in \mathfrak{D}$.

\section{Exemplos.}

a) Dado $\mathcal{F}$ uma folheação singular, a aplicação $p \longmapsto \mathrm{T}_{p} L_{p}$ é uma distribuição singular, dado que $\mathrm{T}_{p} L_{p}=\left\{\left.X\right|_{p} \in \mathrm{T}_{p} M: X \in \mathfrak{X}(\mathcal{F})\right\}$, para todo $p \in M$. Tal distribuição será denotada por $\mathrm{TF}$.

b) Dado $\mathfrak{D} \subset \mathfrak{X}_{\text {loc. }}(M)$ um subconjunto, tal que $\cup_{X \in \mathfrak{D}} \operatorname{Dom}(X)=M$, a associação $p \longmapsto$ $\operatorname{span}\left(\left\{\left.X\right|_{p} \in \mathrm{T}_{p} M: p \in \operatorname{Dom}(X)\right.\right.$ e $\left.\left.X \in \mathfrak{D}\right\}\right)$ é uma distribuição singular a qual será denotada por $\operatorname{span}(\mathfrak{D})$.

Teorema 1.12) (Stefan-Sussmann [32], [31]) Seja $M$ uma variedade diferenciável e $\mathfrak{D} \subset$ $\mathfrak{X}_{\text {loc. }}(M)$ um subconjunto, tal que $\cup_{X \in \mathfrak{D}} \operatorname{Dom}(X)=M$. Considere $\Delta_{\mathfrak{D}}$ a menor distribuição singular $\mathfrak{D}$-invariante, tal que $\operatorname{span}(\mathfrak{D}) \subset \Delta_{\mathfrak{D}}$. Então as $\mathfrak{D}$-órbitas são subvariedades integrais maximais de $\Delta_{\mathfrak{D}}$.

Uma consequência imediata do Teorema de Sussmann é uma condição para integrabilidade da distribuição $\operatorname{span}(\mathfrak{D})$.

Corolário 1.13) Seja $\mathfrak{D} \subset \mathfrak{X}_{\text {loc. }}(M)$, tal que $\cup_{X \in \mathfrak{D}} \operatorname{Dom}(X)=M$ e $\operatorname{span}(\mathfrak{D})$ é $\mathfrak{D}$-invariante. Então as $\mathfrak{D}$-órbitas são subvariedades integrais maximais de $\operatorname{span}(\mathfrak{D})$. 
Além disso, se $\mathfrak{D} \subset \mathfrak{X}(M)$ é um subespaço vetorial, então

$$
\mathrm{T}_{p} \mathfrak{D}(p)=\left\{\left.X\right|_{p} \in \mathrm{T}_{p} M: X \in \mathfrak{D}\right\}
$$

O Teorema de Sussmann pode ser colocado em termos de folheações singulares. Dado que as $\mathfrak{D}$-órbitas são subvariedades integrais maximais da distribuição singular $\Delta_{\mathfrak{D}}$, segue que as $\mathfrak{D}$-órbitas formam uma folheação singular $\mathcal{F}^{\mathfrak{D}}$, tal que $\mathrm{T} \mathcal{F}^{\mathfrak{D}}=\Delta_{\mathfrak{D}}$.

É possível, então, estabelecer-se uma associação $\mathfrak{D} \longmapsto \mathcal{F}^{\mathfrak{D}}$ entre os subconjuntos de campos vetoriais locais, cujos domínios cubram $M$, e as folheações singulares de $M$.

Será mostrado, no corolário a seguir, que esta é uma associação sobrejetora (item (a) do corolário a seguir) que preserva a inclusão (item (c) do corolário a seguir). Ainda, ficará claro, por meio desta sobrejetividade, que toda folheação singular é dada por órbitas de um pseudogrupo, a saber, o pseudogrupo gerado pelo módulo dos campos tangentes a folheação.

\section{Corolário)}

a) Se $\mathcal{F}$ é uma folheação singular, então $\mathcal{F}=\mathcal{F}^{\mathfrak{X}(\mathcal{F})}$.

b) Se $\mathcal{F}$ é uma folheação singular, então dados $L \in \mathcal{F}$ e $p, q \in L$, existem $n \in \mathbb{Z}_{+}^{*}, X \in$ $\mathfrak{X}(\mathcal{F})^{n}, t \in I^{X} \subset \mathbb{R}^{n}$, tal que $p \in D_{t}^{X}$ e $q=\phi_{t}^{X}(p)$.

c) Se $\mathfrak{D} \subset \widetilde{\mathfrak{D}} \subset \mathfrak{X}(M)$, então $\mathcal{F}^{\mathfrak{D}}<\mathcal{F}^{\tilde{\mathfrak{D}}}$. Em particular, se $\mathcal{F}$ e $\widetilde{\mathcal{F}}$ são folheações singulares, tais que $\mathrm{T} \mathcal{F} \subset \mathrm{T} \widetilde{\mathcal{F}}$, então $\mathcal{F}<\widetilde{\mathcal{F}}$.

\section{Demonstração.}

a) Dado que, para cada $X \in \mathfrak{X}(\mathcal{F}), \phi_{t}^{X}$ fixa as folhas de $\mathcal{F}$ (em particular é folheado), segue que $\mathfrak{X}(\mathcal{F})(p) \subset L_{p}$, para todo $p \in M$. Ainda, em decorrência disto, tem-se que $\operatorname{span}(\mathfrak{X}(\mathcal{F}))$ é $\mathfrak{X}(\mathcal{F})$-invariante e, pelo Corolário 1.13 , tem-se que as $\mathfrak{X}(\mathcal{F})$-órbitas são subvariedade integrais maximais de $\operatorname{span}(\mathfrak{X}(\mathcal{F}))$.

Mas, pela definição de folheações singulares, tem-se que $\mathrm{T}_{p} L_{p}=\left.\operatorname{span}(\mathfrak{X}(\mathcal{F}))\right|_{p}$, para todo $p \in M$, de modo que as folhas de $\mathcal{F}$ são subvariedades integrais de $\operatorname{span}(\mathfrak{X}(\mathcal{F}))$.

Assim, pela maximalidade das $\mathfrak{X}(\mathcal{F})$-órbitas, segue que $\mathfrak{X}(\mathcal{F})(p)=L_{p}$, para todo $p \in M$.

b) A afirmação segue como consequência direta da definição de órbitas de subconjuntos de campos vetoriais locais e do item anterior.

c) Segue, imediatamente, da definição de órbitas de um subconjunto de campos vetoriais locais, que

$$
\begin{aligned}
\mathfrak{D}(p) & =\left\{\phi_{t}^{X}(p) \in M: X \in \mathfrak{D}^{n}, t \in I^{X}, n \in \mathbb{Z}_{+}^{*}\right\} \\
& \subset\left\{\phi_{t}^{X}(p) \in M: X \in \widetilde{\mathfrak{D}}^{n}, t \in I^{X}, n \in \mathbb{Z}_{+}^{*}\right\} \\
& =\widetilde{\mathfrak{D}}(p),
\end{aligned}
$$


para todo $p \in M$, ou seja, $\mathcal{F}^{\mathfrak{D}}<\mathcal{F}^{\tilde{\mathfrak{D}}}$.

Ainda, se $\mathcal{F}$ e $\widetilde{\mathcal{F}}$ são folheações singulares, tais que $\mathrm{T} \mathcal{F} \subset \mathrm{T} \widetilde{\mathcal{F}}$, então $\mathfrak{X}(\mathcal{F}) \subset \mathfrak{X}(\widetilde{\mathcal{F}})$ e, pelo item (a), tem-se que

$$
\mathcal{F}=\mathcal{F}^{\mathfrak{X}(\mathcal{F})}<\mathcal{F}^{\mathfrak{X}(\widetilde{\mathcal{F}})}=\widetilde{\mathcal{F}} .
$$

A seção será encerrada mostrando-se que os exemplos apresentados na seção 1.1.1 podem ser facilmente obtidos pela aplicação do Teorema de Stefan-Sussmann a certos submódulos de campos.

\section{Exemplos.}

a) (Folheação homogênea) Dada uma ação de um grupo de Lie $G$ sobre uma variedade $M$, a folheação $\mathcal{F}^{G}$ dada pelas órbitas desta ação é a folheação obtida pela aplicação do Teorema de Stefan-Sussmann ao submódulo dos campos infinitesimais $\mathfrak{X}^{G}(M)$.

b) (Folheação simples) Dada uma submersão $\pi: \bar{M} \longrightarrow M$, a folheação $\mathcal{F}^{\pi}$ dada pelas fibras de $\pi$ é a folheação obtida pela aplicação do Teorema de Stefan-Sussmann ao submódulo dos campos tangentes a distribuição (regular) $\operatorname{Ker}(\mathrm{d} \pi)$.

c) (Folheação de holonomia) Dada uma distribuição horizontal H em um fibrado vetorial $E \stackrel{\pi}{\longrightarrow} M$, a folheação de holonomia $\mathcal{F}^{\mathrm{H}}$ (ver definição na seção 1.1.1) é a folheação obtida pela aplicação do Teorema de Stefan-Sussmann ao submódulo $\mathfrak{X}^{\mathrm{H}}(E)$ dos campos obtidos por levantamento H-horizontal dos campos de $M$. 


\section{Capítulo 2.}

\section{Folheações Riemannianas Singulares (FRS)}

O presente capítulo expõe de modo rudimentar a teoria das folheações Riemannianas singulares (FRS).

O capítulo inicia-se com a Seção 2.1, onde o conceito de FRS será definido a partir da idéia de sistemas transnormais (transnormalidade) e alguns exemplos de FRS serão apresentados (todos relacionados com os exemplos de folheações singulares apresentados na Seção 1.1). Ainda nesta seção, serão expostos os conceitos básicos relacionados a FRS (e.g. seções normais locais, folheação infinitesimal e estratificação) e alguns resultados a cerca dos mesmos.

O capítulo segue com a Seção 2.2, onde será exposto o conceito de linearização em FRS. As idéias presentes nesta seção serão amplamente utilizadas e estarão fortemente presentes no Capítulo 4, onde se encontram os principais resultados deste trabalho. Serão explorados, nesta seção, o lema de homotetita, a linearização de campos, as folheações linearizadas, as extensões homotéticas e alguns resultados relacionando tudo isso.

O capítulo continuará com a Seção 2.3, onde será exposto o conceito de folheação infinitesimal que, grosso modo, é uma FRS, de um espaço Euclideano, invariante pela multiplicação por escalares, para a qual $\{0\}$ é uma folha. A linearização deste tipo de folheação, como será visto, possue uma caracterização interessante, a qual será de grande utilidade no Capítulo 4.

O capítulo será encerrado com a Seção 2.4, onde serão apresentados, brevemente, os conceitos de folheação de tipo órbita e folheação de fecho local.

Enfim, cabe ressaltar que parte significativa deste capítulo baseia-se, em sua concepção, nas seguintes referências [4], [1], [8], [7], [23] e [30].

\subsection{Folheações Riemannianas singulares}

Sejam $(M, g)$ uma variedade Riemanniana. Uma folheação singular $\mathcal{F}$ de $M$ será dita $\underline{\text { Ri- }}$ emanniana se e somente se dado $\gamma$ uma geodésica de $M$, tal que $\gamma^{\prime}\left(t_{0}\right) \in \nu_{\gamma\left(t_{0}\right)} L_{\gamma\left(t_{0}\right)}$, para algum $t_{0} \in \operatorname{Dom}(\gamma)$, tem-se que $\gamma^{\prime}(t) \in \nu_{\gamma(t)} L_{\gamma(t)}$, para todo $t \in \operatorname{Dom}(\gamma)$. Comumente, a tripla $(M, g, \mathcal{F})$ será dita uma variedade Riemanniana folheada e $\mathcal{F}$ será dita uma folheação Riemanniana singular (FRS).

As geodésicas que são ortogonais as todas as folhas de $\mathcal{F}$ serão ditas geodésica $\mathcal{F}$-horizontais ou, simplesmente, horizontais quando ficar claro a folheação a respeito da qual o termo se refere. A condição descrita na definição, de que geodésicas ortogonais a uma folha são sempre horizontais, poderia se aplicar a uma partição qualquer de $M$, não restringindo-se apenas ao caso de folheações singulares. Usualmente no texto, tal condição será referida como condição de 


\section{transnormalidade.}
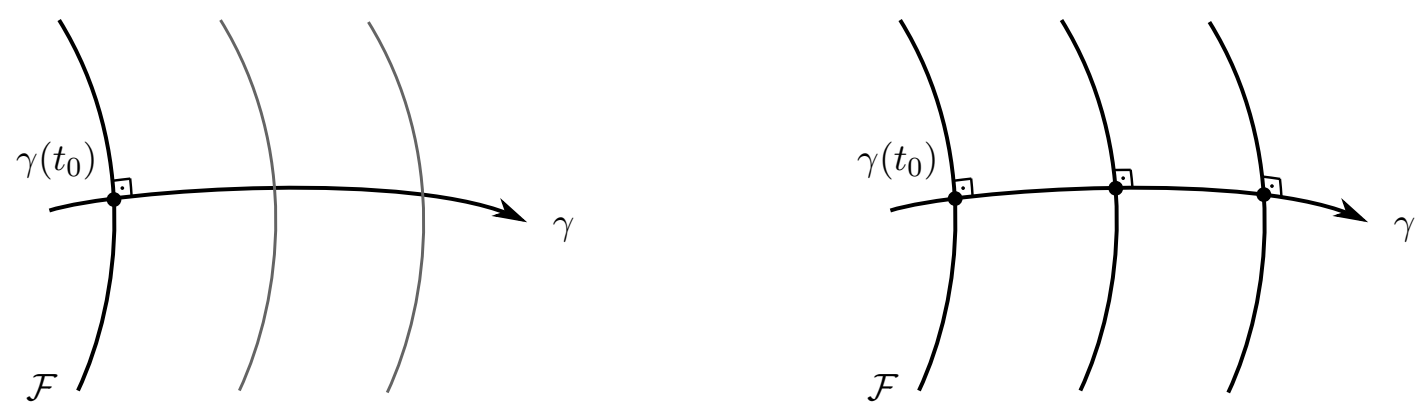

Figura 2.1 : Condição de transnormalidade (a figura à esquerda implica a figura à direita).

\subsubsection{Construções básicas e exemplos de FRS}

A presente seção apresentará exemplos de FRS. Mais precisamente, será mostrado que alguns dos exemplos de folheações singulares apresentados na Seção 1.1.1 são exemplos de FRS, considerando-se, em cada caso, métricas específicas e apropriadas.

Assim, será mostrado que as órbitas de ações ditas isométricas formam uma FRS; será mostrado que a folheação de holonomia em um fibrado vetorial munido com a métrica de Sasaki, cuja definição e as propriedades serão exploradas ao longo da seção, também é uma FRS.

\section{Exemplos triviais - FRS de dimensão máxima e mínima}

Seja $(M, g)$ uma variedade Riemanniana. Então a folheação de $M$ dada pelos seus pontos é uma FRS, pois neste caso todas as geodésicas de $M$ são geodésicas horizontais. Já a folheação de $M$ dada pelo próprio $M$ (o espaço todo) é uma FRS, pois neste caso as geodésicas horizontais de $\{M\}$ são os pontos.

\section{Produto cartesiano de FRS}

Sejam $\left(M, g_{M}, \mathcal{F}_{M}\right)$ e $\left(N, g_{N}, \mathcal{F}_{N}\right)$ variedades Riemannianas folheadas, e considere $\mathcal{F}_{M} \times \mathcal{F}_{N}$ produto das folheações $\mathcal{F}_{M}$ e $\mathcal{F}_{N}$ (ver definição do produto cartesiano de folheações na Seção 1.1.1). Então $\mathcal{F}_{M} \times \mathcal{F}_{N}$ é uma FRS de $\left(M \times N, g_{M} \times g_{N}\right)$, pois dado $\gamma=\left(\gamma_{M}, \gamma_{N}\right)$ uma geodésica de $M \times N$, tal que $\gamma^{\prime}\left(t_{0}\right) \in \nu_{\gamma\left(t_{0}\right)} L_{\gamma\left(t_{0}\right)}^{M \times N}$, para algum $t_{0} \in \operatorname{Dom}(\gamma)$, como

$$
\nu_{(p, q)} L_{(p, q)}^{M \times N}=\nu_{(p, q)}\left(L_{p}^{M} \times L_{q}^{N}\right) \approx \nu_{p} L_{p}^{M} \oplus \nu_{q} L_{q}^{N}
$$

para todo $(p, q) \in M \times N$, tem-se que $\gamma_{M}^{\prime}\left(t_{0}\right) \in \nu_{\gamma_{M}\left(t_{0}\right)} L_{\gamma_{M}\left(t_{0}\right)}^{M}$ e $\gamma_{N}^{\prime}\left(t_{0}\right) \in \nu_{\gamma_{N}\left(t_{0}\right)} L_{\gamma_{N}\left(t_{0}\right)}^{N}$ e, portanto, $\gamma^{\prime}(t) \in \nu_{\gamma(t)} L_{\gamma(t)}^{M \times N}$, para todo $t \in \operatorname{Dom}(\gamma)$. 


\section{Órbitas de ações isométricas (FRS homogêneas)}

Sejam $(M, g)$ uma variedade Riemanniana e $G$ um grupo de Lie, com álgebra de Lie $\mathfrak{g}$. Uma ação suave $\mu: G \times M \longrightarrow M$ é dita isométrica se e somente se $\mu^{g} \in \operatorname{Iso}(M, g)$, onde $\mu^{g}:=\mu(g, \cdot)$, para todo $g \in G$.

Relembre, do exemplo de folheações homogêneas, na Seção 1.1.1, que dado $X \in \mathfrak{X}(M)$, o campo infinitesimal $X^{*} \in \mathfrak{X}(M)$ dado por

$$
\left.X^{*}\right|_{p}:=\left.\frac{\mathrm{d}}{\mathrm{d} t} \mu(\exp (t X), p)\right|_{t=0}=\left.\mathrm{d} \mu_{p}\right|_{e}(X)
$$

era suave e o plano tangente a órbita $G(p)$, no ponto $p$, era gerado por estes campos.

Lema) Seja $\mu$ uma ação isométrica. Então, para cada $X \in \mathfrak{X}(M)$,

a) $\quad X^{*}$ é um campo de Killing.

b) Dado $\gamma$ uma geodésica de $M,\left\langle X^{*} \circ \gamma, \gamma^{\prime}\right\rangle$ é constante.

\section{Demonstração.}

a) Seja $X \in \mathfrak{X}(M)$ e considere $\phi$ o fluxo de $X^{*}$.

Segue, da definição de campo infinitesimal, que $t \longmapsto \mu^{\exp (t X)}(p)$ é a curva integral de $X^{*}$ passando por $p$ no instante $t=0$, pois

$$
\begin{aligned}
\frac{\mathrm{d}}{\mathrm{d} t} \mu^{\exp (t X)}(p) & =\left.\frac{\mathrm{d}}{\mathrm{d} s} \mu^{\exp ((s+t) X)}(p)\right|_{s=0} \\
& =\left.\frac{\mathrm{d}}{\mathrm{d} s} \mu(\exp (s X) \exp (t X), p)\right|_{s=0} \\
& =\left.\frac{\mathrm{d}}{\mathrm{d} s} \mu(\exp (s X), \mu(\exp (t X), p))\right|_{s=0} \\
& =\left.X^{*}\right|_{\mu^{\exp (t X)}(p)}
\end{aligned}
$$

Assim $\phi_{t}=\mu^{\exp (t X)}$, para todo $t \in \mathbb{R}$ e, portanto, o fluxo de $X^{*}$ é dado por isometrias, ou seja, $X^{*}$ é um campo de Killing.

b) Seja $\gamma$ uma geodésica em $M$.

Uma vez que $X^{*}$ é um campo de Killing, segue que

$$
2\left\langle\frac{\mathrm{D}}{\mathrm{d} t} X^{*} \circ \gamma, \gamma^{\prime}\right\rangle=\left\langle\nabla_{\gamma^{\prime}} X^{*}, \gamma^{\prime}\right\rangle+\left\langle\gamma^{\prime}, \nabla_{\gamma^{\prime}} X^{*}\right\rangle=0
$$

e, pela compatibilidade da métrica com a conexão Riemanniana, tem-se que

$$
\frac{\mathrm{d}}{\mathrm{d} t}\left\langle X^{*} \circ \gamma, \gamma^{\prime}\right\rangle=\left\langle\frac{\mathrm{D}}{\mathrm{d} t} X^{*} \circ \gamma, \gamma^{\prime}\right\rangle+\left\langle X^{*} \circ \gamma, \frac{\mathrm{D} \gamma^{\prime}}{\mathrm{d} t}\right\rangle=0
$$

ou seja, $\left\langle X^{*} \circ \gamma, \gamma^{\prime}\right\rangle$ é constante. 
Proposição) Seja $\mu$ uma ação isométrica. Então a folheação (singular) $\mathcal{F}^{G}$, dada pelas órbitas de $\mu$, é uma FRS.

Demonstração. Já foi demonstrado, na Proposição 1.5 , que $\mathcal{F}^{G}$ é uma folheação. Será demonstrado, então, que tal folheação é Riemanniana.

Seja $\gamma$ uma geodésica em $M$, tal que $\gamma^{\prime}\left(t_{0}\right) \in \nu_{\gamma\left(t_{0}\right)} G\left(\gamma\left(t_{0}\right)\right)$, para algum $t_{0} \in \operatorname{Dom}(\gamma)$.

Dado $t \in \operatorname{Dom}(\gamma)$ e $v \in \mathrm{T}_{\gamma(t)} G(\gamma(t))$ considere $X \in \mathfrak{g}$, tal que $\left.X^{*}\right|_{\gamma(t)}=v$.

Uma vez que $\left\langle\left. X^{*}\right|_{\gamma\left(t_{0}\right)}, \gamma^{\prime}\left(t_{0}\right)\right\rangle=0$, pois $\left.X^{*}\right|_{\gamma\left(t_{0}\right)} \in \mathrm{T}_{\gamma\left(t_{0}\right)} G\left(\gamma\left(t_{0}\right)\right)$ e $\gamma^{\prime}\left(t_{0}\right) \in \nu_{\gamma\left(t_{0}\right)} G\left(\gamma\left(t_{0}\right)\right)$, pelo lema anterior, segue que $\left\langle X^{*} \circ \gamma, \gamma^{\prime}\right\rangle=0$.

Assim $\left\langle v, \gamma^{\prime}(t)\right\rangle=0$, para todo $v \in \mathrm{T}_{\gamma(t)} G(\gamma(t))$ e $t \in \operatorname{Dom}(\gamma)$ e, portanto, $\gamma^{\prime}(t) \in$ $\nu_{\gamma(t)} G(\gamma(t))$, para todo $t \in \operatorname{Dom}(\gamma)$.

\section{Folheação de holonomia}

Sejam $\left(M, g_{M}\right)$ uma variedade Riemanniana conexa, $E$ um fibrado vetorial Euclidiano sobre $M$, com projeção $\pi$ (i.e. um fibrado vetorial com um produto interno $\langle\cdot, \cdot\rangle \in \Gamma\left(E^{*} \otimes E^{*}\right)$ nas fibras de $E$ ). Uma distribuição horizontal $\mathrm{H} \subset \mathrm{T} E$ será dita métrica se e somente se a conexão linear $\nabla: \mathfrak{X}(M) \times \Gamma(E) \longrightarrow \Gamma(E)$ induzida por $\mathrm{H}$ é compatível com o produto interno de $E$ (i.e. $X\left(\left\langle S, S^{\prime}\right\rangle\right)=\left\langle\nabla_{X} S, S^{\prime}\right\rangle+\left\langle S, \nabla_{X} S^{\prime}\right\rangle$, para todo $X \in \mathfrak{X}(M)$ e $S, S^{\prime} \in \Gamma(E)$ ).

Dada $\mathrm{H} \subset \mathrm{T} E$ uma distribuição horizontal métrica, para cada $\alpha \in C_{\mathrm{par} .}^{\infty}([0,1], M)$, será considerado $\mathrm{P}_{\alpha}: E_{\alpha(0)} \longrightarrow E_{\alpha(1)}$ o operador de transporte paralelo ao longo de $\alpha$. A saber, $\mathrm{P}_{\alpha}$ é uma isometria, dado a compatibilidade de $\nabla$ com o produto interno de $E$. Ainda, será denotado por $\pi_{\mathrm{V}}: \mathrm{T} E \longrightarrow E$ a projeção vertical induzida por $\mathrm{H}$, ou seja, a projeção relativa a decomposição $\mathrm{T} E=\operatorname{Ker}(\mathrm{d} \pi) \oplus \mathrm{H}$, onde $\operatorname{Ker}(\mathrm{d} \pi)$ é identificado fibra à fibra com $E$, no contradomínio de $\pi_{\mathrm{v}}$.

Neste contexto, a aplicação $g^{E}: E \longrightarrow \mathrm{T} E^{*} \otimes \mathrm{T} E^{*}$ dada por $g^{E}:=\pi^{*} g_{M} \oplus \pi_{\mathrm{v}}^{*}\langle\cdot, \cdot\rangle$, ou seja, dada por

$$
\left.g^{E}\right|_{x}(V, W):=\left.g_{M}\right|_{\pi(x)}\left(\left.\mathrm{d} \pi\right|_{x}(V),\left.\mathrm{d} \pi\right|_{x}(W)\right)+\left\langle\left.\pi_{\mathrm{v}}\right|_{x}(V),\left.\pi_{\mathrm{v}}\right|_{x}(W)\right\rangle_{\pi(x)}
$$

é uma métrica em $E$ denominada a métrica de Sasaki relativa à distribuição horizontal métrica $\mathrm{H}$.

A seguir serão expressas as relações entre os campos horizontais e os campos verticais (i.e. tangentes a $\operatorname{Ker}(\mathrm{d} \pi)$ ) dentro da métrica de Sasaki, mostrando que essas relações são suficientes para caracterizar tal métrica.

Lema) Seja $\mathrm{H} \subset \mathrm{T} E$ uma distribuição horizontal métrica. Então

a) $\left.g^{E}\right|_{x}(V, W)=\langle V, W\rangle_{\pi(x)}$, para todo $V, W \in \operatorname{Ker}\left(\left.\mathrm{d} \pi\right|_{x}\right)$.

b) $\left.g^{E}\right|_{x}(X, V)=0$, para todo $X \in \mathrm{H}_{x}$ e $V \in \operatorname{Ker}\left(\left.\mathrm{d} \pi\right|_{x}\right)$.

c) $\left.g^{E}\right|_{x}(X, Y)=\left.\pi^{*} g_{M}\right|_{x}(X, Y)$, para todo $X, Y \in \mathrm{H}_{x}$. 
d) Se uma métrica $g$, em $E$, satisfaz os itens anteriores, então $g=g^{E}$.

e) $\pi:\left(E, g^{E}\right) \longrightarrow\left(M, g_{M}\right)$ é uma submersão Riemanniana.

Demonstração. Os itens (a), (b) e (c) decorrem diretamente da definição da métrica de Sasaki.

O item (d) decorre da decomposição $\mathrm{T} E=\operatorname{Ker}(\mathrm{d} \pi) \oplus \mathrm{H}$ e da bilinearidade de $g$ e $g^{E}$.

e) Afirma-se que $\operatorname{Ker}\left(\left.\mathrm{d} \pi\right|_{x}\right)^{\perp}=\mathrm{H}_{x}$. De fato, se $X \in\left(\left.\operatorname{Ker} \mathrm{d} \pi\right|_{x}\right)^{\perp}$, então

$$
\begin{aligned}
0 & =\left.g^{E}\right|_{x}(X, V) \\
& =\left.g_{M}\right|_{\pi(x)}\left(\left.\mathrm{d} \pi\right|_{x}(X),\left.\mathrm{d} \pi\right|_{x}(V)\right)+\left\langle\left.\pi_{\mathrm{V}}\right|_{x}(X),\left.\pi_{\mathrm{V}}\right|_{x}(V)\right\rangle_{\pi(x)} \\
& =\left\langle\left.\pi_{\mathrm{v}}\right|_{x}(X), V\right\rangle_{\pi(x)},
\end{aligned}
$$

para todo $V \in \operatorname{Ker}\left(\left.\mathrm{d} \pi\right|_{x}\right)$ e, como $\left.\pi_{\mathrm{v}}\right|_{x}(X) \in=\operatorname{Ker}\left(\left.\mathrm{d} \pi\right|_{x}\right)$, segue que $\left.\pi_{\mathrm{v}}\right|_{x}(X)=0$, ou seja, $X \in \operatorname{Ker}\left(\left.\pi_{\mathrm{v}}\right|_{x}\right)=\mathrm{H}_{x}$. Logo $\operatorname{Ker}\left(\left.\mathrm{d} \pi\right|_{x}\right)^{\perp} \subset \mathrm{H}_{x}$ e, $\operatorname{como} \operatorname{dim}\left(\operatorname{Ker}\left(\left.\mathrm{d} \pi\right|_{x}\right)^{\perp}\right)=$ $\operatorname{dim}\left(\mathrm{H}_{x}\right)$, decorre que $\operatorname{Ker}\left(\left.\mathrm{d} \pi\right|_{x}\right)^{\perp}=\mathrm{H}_{x}$.

Assim, para todo $X, Y \in \operatorname{Ker}\left(\left.\mathrm{d} \pi\right|_{x}\right)^{\perp}$, tem-se que $\left.g^{E}\right|_{x}(X, Y)=\left.\pi^{*} g_{M}\right|_{x}(X, Y)$, ou seja, $\pi$ é uma submersão Riemanniana.

Cabe observar que, na demonstração do item (e), fica claro que a métrica de Sasaki torna $\mathrm{H}$ a distribuição horizontal da submersão Riemanniana $\pi$, o que de certo modo mostra a "naturalidade" desta métrica. Assim, a partir deste ponto, não será feita distinção entre H e a distribuição horizontal da submersão Riemanniana $\pi$.

A seguir, na Proposição 2.2, serão exibidas algumas propriedades métricas das fibras de $E$, quando o fibrado estiver munido da métrica de Sasaki. Entretanto, para a demonstrar tais propriedades, faz-se necessário o uso do Lema 2.1, que apresentará condições necessárias e suficientes para que as fibras de uma submersão Riemanniana qualquer sejam totalmente geodésicas (em particular, tal lema se aplicará a projeção $\pi$ ).

Lema 2.1) Seja $\pi: \widetilde{M} \longrightarrow M$ uma submersão Riemanniana. Então as fibras de $E$ serão totalmente geodésicas se e somente se $\mathrm{P}_{\alpha}^{t}: \pi^{-1}(\alpha(0)) \longrightarrow \pi^{-1}(\alpha(t))$ é uma isometria, onde $\mathrm{P}_{\alpha}^{t}$ é o transporte paralelo ao longo de $\alpha$ com relação a distribuição horizontal $\operatorname{Ker}(\mathrm{d} \pi)^{\perp}$, para todo $\alpha \in C^{\infty}([0,1] ; M)$.

Demonstração. Considere $\tilde{g}$ a métrica de $E$ e $\widetilde{\nabla}$ a conexão Riemanniana de $(\widetilde{M}, \tilde{g})$. Denote $\mathrm{H}:=\operatorname{Ker}(\mathrm{d} \pi)^{\perp}$ e, para cada $p \in M$, denote $\widetilde{M}_{p}=\pi^{-1}(p)$ e observe que $\mathrm{H}_{x}=\left(\mathrm{T}_{x} \widetilde{M}_{\pi(x)}\right)^{\perp}=$ $\nu_{x} \widetilde{M}_{\pi(x)}$.

Sejam

- $p \in M, x \in E_{p}, V \in \mathrm{T}_{x} E_{p}$ e $\gamma_{p}$ uma curva em $E_{p}$, tal que $\gamma_{p}(0)=x$ e $\gamma_{p}^{\prime}(0)=V$; 
- $X \in \mathrm{H}_{x}$ e $\alpha \in C^{\infty}([0,1] ; M)$, tal que $X=\left.\frac{\mathrm{d}}{\mathrm{d} t} \mathrm{P}_{\alpha}^{t}\right|_{t=0}$.

Defina $\varphi: \operatorname{Dom}\left(\gamma_{p}\right) \times[0,1] \subset \mathbb{R}^{2} \longrightarrow E$, por $\varphi(s, t):=\mathrm{P}_{\alpha}^{t}\left(\gamma_{p}(s)\right)$. Então

$$
\left.\partial_{s} \varphi\right|_{\varphi(s, t)}=\left.\mathrm{dP}_{\alpha}^{t}\right|_{\gamma_{p}(s)}\left(\gamma_{p}^{\prime}(s)\right) \in \mathrm{T}_{\varphi(s, t)} E_{\alpha(t)},\left.\quad \partial_{t} \varphi\right|_{\varphi(s, t)}=\frac{\mathrm{d}}{\mathrm{d} t} \mathrm{P}_{\alpha}^{t}\left(\gamma_{p}(s)\right) \in \mathrm{H}_{\varphi(s, t)},
$$

para todo $(s, t) \in \operatorname{Dom}\left(\gamma_{p}\right) \times[0,1]$.

Segue que $g\left(\partial_{t} \varphi, \partial_{s} \varphi\right)=0$ e, pela compatibilidade de $\nabla$ com a métrica $g$, tem-se que

$$
\begin{aligned}
g\left(\frac{\mathrm{D}}{\mathrm{d} s} \partial_{t} \varphi, \partial_{s} \varphi\right) & =-g\left(\partial_{t} \varphi, \frac{\mathrm{D}}{\mathrm{d} s} \partial_{s} \varphi\right) \\
& =-g\left(\partial_{t} \varphi, \nabla_{\partial_{s} \varphi} \partial_{s} \varphi\right) \\
& =-g\left(\partial_{t} \varphi,\left(\nabla_{\partial_{s} \varphi} \partial_{s} \varphi\right)^{\perp}\right) \\
& =-g\left(\partial_{t} \varphi, \mathrm{II}^{\alpha(t)}\left(\partial_{s} \varphi, \partial_{s} \varphi\right)\right)
\end{aligned}
$$

onde $\mathrm{II}^{\alpha(t)} \in \Gamma\left(\mathrm{T} E_{\alpha(t)}^{*} \otimes \mathrm{T} E_{\alpha(t)}^{*} \otimes \nu E_{\alpha(t)}\right)$ é a segunda forma fundamental de $E_{\alpha(t)}$.

Logo, pela simetria d $\nabla$, tem-se que

$$
\partial_{t}\left\|\partial_{s} \varphi\right\|^{2}=2 g\left(\frac{\mathrm{D}}{\mathrm{d} t} \partial_{s} \varphi, \partial_{s} \varphi\right)=2 g\left(\frac{\mathrm{D}}{\mathrm{d} s} \partial_{t} \varphi, \partial_{s} \varphi\right)=-2 g\left(\partial_{t} \varphi, \mathrm{II}^{\alpha(t)}\left(\partial_{s} \varphi, \partial_{s} \varphi\right)\right) .
$$

Assim, se $s=0$, segue que

$$
\frac{\mathrm{d}}{\mathrm{d} t}\left\|\left.\mathrm{dP}_{\alpha}^{t}\right|_{x}(V)\right\|^{2}=-2 g\left(\widetilde{\alpha}_{x}^{\prime}(t), \mathrm{II}^{\alpha(t)}\left(\left.\mathrm{dP}_{\alpha}^{t}\right|_{x}(V),\left.\mathrm{dP}_{\alpha}^{t}\right|_{x}(V)\right)\right)
$$

Se $\mathrm{P}_{\alpha}^{t}$ é uma isometria, para todo $\alpha \in C^{\infty}([0,1] ; M)$ e $t \in \operatorname{Dom}\left(\widetilde{\alpha}_{x}\right)$, por 2.1 , tem-se que

$$
g\left(\frac{\mathrm{d}}{\mathrm{d} t} \mathrm{P}_{\alpha}^{t}(x), \mathrm{II}^{\alpha(t)}\left(\left.\mathrm{dP}_{\alpha}^{t}\right|_{x}(V),\left.\mathrm{dP}_{\alpha}^{t}\right|_{x}(V)\right)\right)=0
$$

e, portanto, se $t=0$, segue que $g\left(X, \mathrm{II}^{p}(V, V)\right)=0$. Logo II ${ }^{p} \equiv 0$, ou seja, a fibra $E_{p}$ é totalmente geodésica.

Então, como a escolha de $p \in M$ foi arbitrária, segue que as fibras de $\pi$ são totalmente geodésicas.

Se as fibras de $\pi$ são totalmente geodésicas, segue que $\mathrm{II}^{\alpha(t)} \equiv 0$, para todo $t \in \operatorname{Dom}\left(\widetilde{\alpha}_{x}\right)$ e, por 2.1, tem-se que $\left\|\left.\mathrm{dP}_{\alpha}^{t}\right|_{x}(V)\right\|$ é constante. Logo

$$
\left\|\left.\mathrm{dP}_{\alpha}^{t}\right|_{x}(V)\right\|=\left\|\left.\mathrm{dP}_{\alpha}^{0}\right|_{x}(V)\right\|=\|V\|
$$

ou seja, $\mathrm{P}_{\alpha}^{t}$ é uma isometria.

Então, como a escolha de $\alpha$ dependeu de escolhas arbitrárias, a saber, da escolha de $p \in M$ e $X \in \mathrm{H}_{x}$, segue que $\mathrm{P}_{\alpha}^{t}$ é uma isometria, para toda $\alpha \in C^{\infty}([0,1] ; M)$.

Proposição 2.2) Seja $\mathrm{H} \subset \mathrm{T} E$ uma distribuição horizontal métrica. Então, para cada $p \in M$, 
a) $\langle\cdot, \cdot\rangle_{p} \equiv\left(i_{p}\right)^{*} g E$, identificando-se $E_{p}$ com $\mathrm{T}_{x} E_{p}$, para todo $x \in E_{p}$, onde $i_{p}: E_{p} \longrightarrow E$ é a inclusão. Em particular, $E_{p}$ é uma subvariedade plana;

b) $E_{p}$ é uma subvariedade totalmente geodésica de $E$.

\section{Demonstração.}

a) Uma vez que $\pi \circ i_{p}$ é constante e igual a $p$, tem-se que $\left.\left.\mathrm{d} \pi\right|_{i_{p}(x)} \circ \mathrm{d} i_{p}\right|_{x}=0$, ou seja, $\left.\mathrm{d} i_{p}\right|_{x}\left(E_{p}\right) \subset \operatorname{Ker}\left(\left.\mathrm{d} \pi\right|_{i_{p}(x)}\right)$, para todo $x \in E_{p}$.

Assim

$$
\begin{aligned}
\left.\left(i_{p}\right)^{*} g^{E}\right|_{x}(V, W)= & \left.g^{E}\right|_{i_{p}(x)}\left(\left.\mathrm{d} i_{p}\right|_{x}(V),\left.\mathrm{d} i_{p}\right|_{x}(W)\right) \\
= & \left.g_{M}\right|_{\pi\left(i_{p}(x)\right)}\left(\left.\mathrm{d} \pi\right|_{i_{p}(x)}\left(\left.\mathrm{d} i_{p}\right|_{x}(V)\right),\left.\mathrm{d} \pi\right|_{i_{p}(x)}\left(\left.\mathrm{d} i_{p}\right|_{x}(W)\right)\right)+ \\
& +\left\langle\left.\pi_{\mathrm{V}}\right|_{i_{p}(x)}\left(\left.\mathrm{d} i_{p}\right|_{x}(V)\right),\left.\pi_{\mathrm{V}}\right|_{i_{p}(x)}\left(\left.\mathrm{d} i_{p}\right|_{x}(W)\right)\right\rangle_{\pi(x)} \\
= & \langle V, W\rangle_{p},
\end{aligned}
$$

para todo $x \in E$ e $V, W \in \mathrm{T}_{x} E_{p} \approx E_{p}$, ou seja, $\langle\cdot, \cdot\rangle_{p} \equiv\left(i_{p}\right)^{*} g^{E}$.

b) Dado $\alpha \in C^{\infty}([0,1] ; M)$, tem-se que $\mathrm{P}_{\alpha}^{t}$ é uma isometria, para todo $t \in \operatorname{Dom}(\alpha)$. De fato, como $\nabla$ é compatível com $\langle\cdot, \cdot\rangle$, tem-se que $\mathrm{P}_{\alpha}^{t}$ é uma isometria linear, ou seja, $\langle V, W\rangle_{\alpha(0)}=\left\langle\mathrm{P}_{\alpha}^{t}(V), \mathrm{P}_{\alpha}^{t}(W)\right\rangle_{\alpha(t)}$, para todo $V, W \in E_{\alpha(0)}$. Logo, pelo item anterior, que $\mathrm{P}_{\alpha}^{t}$ é uma isometria, ou seja,

$$
\left.\left(i_{\alpha(0)}\right)^{*} g^{E}\right|_{x}(V, W)=\left.\left(i_{\alpha(t)}\right)^{*} g^{E}\right|_{\mathrm{P}_{\alpha}^{t}(x)}\left(\left.\mathrm{dP}_{\alpha}^{t}\right|_{x}(V),\left.\mathrm{dP}_{\alpha}^{t}\right|_{x}(W)\right)
$$

para todo $x \in E_{\alpha(0)}$ e $V, W \in \mathrm{T}_{x} E_{\alpha(0)} \approx E_{\alpha(0)}$.

Assim, como $\pi:(E, g E) \longrightarrow\left(M, g_{M}\right)$ é uma submersão Riemanniana, segue pelo lema anterior que as fibras de $E$ são totalmente geodésicas.

Dada $\mathrm{H} \subset \mathrm{T} E$ uma distribuição horizontal métrica, considere $\mathcal{F}^{\mathrm{H}}$ a folheação de holonomia da distribuição H (ver Seção 1.1.1), cuja folha passando por $x$, a saber, era dada por

$$
L_{x}:=\left\{\mathrm{P}_{\alpha}(x) \in E: \alpha \in C_{\text {par. }}^{\infty}([0,1], M): \alpha(0)=x\right\}
$$

Lema 2.3) Seja $H \subset T E$ uma distribuição horizontal métrica. Então

a) $\nu_{x} L_{x} \subset \operatorname{Ker}\left(\left.\mathrm{d} \pi\right|_{x}\right) \approx E_{\pi(x)}$, para todo $x \in E$,

b) $\nu_{0_{p}} L_{0_{p}}=\operatorname{Ker}\left(\left.\mathrm{d} \pi\right|_{0_{p}}\right) \approx E_{p}$, para todo $p \in M$.

\section{Demonstração.}

a) Segue, do item (c) da Lema 1.7, que $\nu_{x} L_{x}=\left(\mathrm{T}_{x} L_{x}\right)^{\perp} \subset\left(\mathrm{H}_{x} E\right)^{\perp}=\mathrm{V}_{x} E$. 
b) Segue, do item anterior, que $\nu_{0_{p}} L_{0_{p}} \subset \operatorname{Ker}\left(\left.\mathrm{d} \pi\right|_{0_{p}}\right)$. Mas, pelo item (d) do Lema 1.7, tem-se que

$$
\operatorname{dim}\left(\operatorname{Ker}\left(\left.\mathrm{d} \pi\right|_{0_{p}}\right)\right)=\operatorname{dim}(E)-\operatorname{dim}(M)=\operatorname{dim}(E)-\operatorname{dim}\left(\mathrm{T}_{0_{p}} L_{0_{p}}\right)=\operatorname{dim}\left(\nu_{0_{p}} L_{0_{p}}\right) .
$$

$\operatorname{Assim} \nu_{0_{p}} L_{0_{p}}=\operatorname{Ker}\left(\left.\mathrm{d} \pi\right|_{0_{p}}\right)$.

Proposição 2.4) Seja $\mathrm{H} \subset \mathrm{T} E$ uma distribuição horizontal métrica. Então $\mathcal{F}^{\mathrm{H}}$ é uma FRS de $\left(E, g^{E}\right)$, onde $g^{E}$ é a métrica de Sasaki de $E$.

Demonstração. Seja $\gamma$ uma geodésica de $E$, tal que $\gamma^{\prime}\left(t_{0}\right) \in \nu_{\gamma\left(t_{0}\right)} L_{\gamma\left(t_{0}\right)}$, para algum $t_{0} \in$ $\operatorname{Dom}(\gamma)$ e denote $\gamma\left(t_{0}\right)=x$ e $\pi(x)=p$. Segue, pelo item (a) do lema anterior, que $\gamma^{\prime}\left(t_{0}\right) \in E_{p}$.

Considere $r: \mathbb{R} \longrightarrow E_{p}$ a reta dada por $r(t):=x+\left(t-t_{0}\right) \gamma^{\prime}\left(t_{0}\right)$. Uma vez que $E_{p}$ é plana (ver item (a) da Proposição 2.2), tem-se que $r$ é uma geodésica de $E_{p}$. Ainda, como $E_{p} \subset E$ é uma subvariedade totalmente geodésica, pelo item (b) da Proposição 2.2, tem-se que $r$ é uma geodésica de $E$, com $r\left(t_{0}\right)=x$ e $r^{\prime}\left(t_{0}\right)=\gamma^{\prime}\left(t_{0}\right)$.

Então, pela unicidade das geodésicas passando por $x$ com velocidade $\gamma^{\prime}\left(t_{0}\right)$, tem-se que $\gamma$ é um segmento de reta em $E_{p}$, ou seja, $\gamma$ é uma geodésica em $E_{p}$.

Mas $\gamma^{\prime}\left(t_{0}\right) \in \nu_{x} L_{x}$ e $\gamma^{\prime}\left(t_{0}\right) \in E_{p}$ e, portanto,

$$
\gamma^{\prime}\left(t_{0}\right) \in \nu_{x}\left(L_{x} \cap E_{p}\right)=\nu_{\gamma\left(t_{0}\right)} \operatorname{Hol}_{p}\left(\gamma\left(t_{0}\right)\right)
$$

Logo, como $\left\{\operatorname{Hol}_{p}(y)\right\}_{y \in E_{p}}$ é uma FRS em $E_{p}$, segue que $\gamma^{\prime}(t) \in \nu_{\gamma(t)} \operatorname{Hol}_{p}(\gamma(t))=\nu_{\gamma(t)}\left(L_{\gamma(t)} \cap\right.$ $\left.E_{p}\right)$, para todo $t \in \operatorname{Dom}(\gamma)$.

Assim $\gamma^{\prime}(t) \in \nu_{\gamma(t)} L_{\gamma(t)}$, para todo $t \in \operatorname{Dom}(\gamma)$.

\subsubsection{Seções normais locais e vizinhanças tubulares normais (VTN)}

Sejam $(M, g)$ uma variedade Riemanniana e $\mathcal{F}$ uma FRS de $M$. A estrutura Riemanniana da folheação $\mathcal{F}$ permitirá considerar, seções transversais locais com propriedades geométricas adicionais, além da transversalidade (propriedade topológica) apresentada na seção 1.1.2.

Lema) (Seção normal local) Seja $p \in M$. Então, existe $U_{p} \subset \operatorname{Dom}\left(\exp _{p}\right) \cap \nu_{p} L_{p}$ aberto, tal que $\left.\exp _{p}\right|_{U_{p}}$ é um difeomorfismo (sobre a sua imagem) e a subvariedade $S_{p}:=\exp _{p}\left(U_{p}\right)$ é uma seção transversal local de $\mathcal{F}$ passando por $p, \operatorname{com} \mathrm{T}_{p} S_{p}=\nu_{p} L_{p}$.

Demonstração. Dado que $\left.\exp _{p}\right|_{U_{p}}$ é um difeomorfismo, é imediato ver que $S_{p}:=\exp _{p}\left(U_{p}\right)$ é uma subvariedade mergulhada, com $\left.\mathrm{T}_{q} S_{p} \approx \operatorname{dexp}_{p}\right|_{\exp _{p}^{-1}(q)}\left(\nu_{p} L_{p}\right)$, para todo $q \in S_{p}$ e, em paricular, $\mathrm{T}_{p} S_{p}=\nu_{p} L_{p}$, de modo que $\mathrm{T}_{p} S_{p} \oplus \mathrm{T}_{p} L_{p}=\mathrm{T}_{p} M$ é uma decomposição ortogonal.

Assim, diminuido-se $S_{p}$ (equivalentemente diminuindo-se $U_{p}$ ), tem-se que $S_{p}$ é uma seção transversal local de $\mathcal{F}$ passando por $p$. 
Uma seção transversal local como descrita no lema anterior será dita um "slice" normal ou uma seção normal local de $\mathcal{F}$ passando por $p$. Ainda, para cada $\varepsilon>0$, tal que $\left.\exp _{p}\right|_{\nu_{p}^{\varepsilon} L_{p}}$ é um difeomorfismo, onde $\nu_{p}^{\varepsilon} L_{p}:=\left\{x \in \nu_{p} L_{p}:\|x\|_{p}<\varepsilon\right\}$, a seção normal local $S_{p}^{\varepsilon}:=\exp _{p}\left(\nu_{p}^{\varepsilon} L_{p}\right)$ será dita um "slice" normal de raio $\varepsilon$ ou uma seção normal de raio $\varepsilon$.

Seja $B \subset M$ uma subvariedade mergulhada. Considere $\nu B$ o fibrado normal de $B$ e $\mathcal{W} \subset \nu B$ um aberto em torno da seção nula de $\nu B$, tal que

- $\mathcal{W} \subset \operatorname{Dom}(\exp )$

- $\left.\exp \right|_{\mathcal{W}}$ é um difeomorfismo sobre a sua imagem,

- $d(\exp (\eta), B)=\|\eta\|$, para todo $\eta \in \mathcal{W}$, ou seja, a distância entre $\exp (\eta)$ e $B$ é realizada pela geodésica radial $t \longmapsto \exp (t \eta)$.

Então o conjunto $W=\exp (\mathcal{W})$ será dito uma vizinhança tubular normal, em torno de $B$. Ainda, se $\mathcal{W}=\nu^{\varepsilon} B=\{\eta \in \nu B:\|\eta\|<\varepsilon\}$ a vizinhança tubular normal $W$ será dita uma vizinhança tubular normal de raio $\varepsilon$ ou, simplificadamente, uma $\varepsilon$-vizinhança tubular.

É um fato bem conhecido (ver a Proposição 26 da página 200 de [27] ou o Corolário 5.5.3 de [28], por exemplo) que se $B \subset M$ for fechada ou, equivalentemente, propriamente mergulhada, então sempre existe uma vizinhança tubular normal em torno de $B$. Ainda, se $B$ for compacta, então sempre existe uma $\varepsilon$-vizinhança tubular em torno de $B$.

Cabe ressaltar que uma vizinhança tubular normal $W=\exp (\mathcal{W})$ é sempre um aberto em torno de $B$, dado que é imagem de um aberto por um difeomorfismo. Além disso, $W$ também é uma fibração sobre $B$, com projeção $\rho: W \longrightarrow B$ caracterizada por $\rho \circ \exp =\pi$ e cuja fibra sobre $b \in B$ é dada por $W_{b}=\exp _{b}\left(\mathcal{W}_{b}\right)$. A título de ilustração, quando $B$ é uma folha de $\mathcal{F}$ as fibras dessa fibração são dadas por seções normais locais.

Observação) (Relação com o conceito topológico de vizinhança tubular) Similarmente ao caso das VTD's, as vizinhanças tubulares normais, ao contrário do que sugere o nome, não são vizinhanças tubulares. Elas são, na verdade, vizinhanças tubulares parciais (ver observação da Seção 1.1.3).

A seguir serão listados dois resultados, pertinentes a este texto, a cerca da existência de $\varepsilon$-vizinhanças tubulares, com propriedades especiais.

O primeiro caracteriza as FRS como sendo as folheações singulares para as quais sempre existem $\varepsilon$-vizinhanças tubulares, em torno de abertos das folhas, dentro das quais as folhas são equidistantes (ver a Proposição 2.3 em [30] ou a Proposição 2.4.3 em [29], para a demonstração, por exemplo).

O segundo é um resultado conhecida da área de FRS, que está bem demonstrado em [30] (Proposição 2.31) ou [21] (Proposição 16), por exemplo.

Proposição 2.5) (Equidistância local de FRS) Sejam $M$ uma variedade Riemanniana e $\mathcal{F}$ uma folheação singular de $M$. Então $\mathcal{F}$ é Riemanniana se e somente se, para cada $p \in M$, existe $P \subset L_{p}$ uma vizinhança aberta de $p$ e $W$ uma $\varepsilon$-vizinhança tubular em torno de $P$, tal 
que $\left.\mathcal{F}\right|_{W}$ é uma folheação singular equidistante (i.e. dado $\left.L^{W} \in \mathcal{F}\right|_{W}, d(q, P)$ é constante, para todo $\left.q \in L^{W}\right)$.

Proposição 2.6) Sejam $M$ uma variedade Riemanniana, $\mathcal{F}$ uma FRS de $M$ e $L \in \mathcal{F}$ fechada. Então existe uma $\varepsilon$-vizinhança tubular em torno de $L$.

\subsubsection{Dimensão das folhas e estratificação}

Sejam $(M, g)$ uma variedade Riemanniana e $\mathcal{F}$ uma FRS de $M$. Dado $k \in \mathbb{N}$, cada compo-

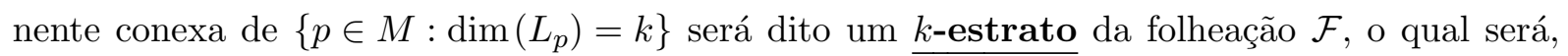
genericamente, denotado por $\Sigma_{k}$.

Quando $k=\operatorname{mín} .\left\{\operatorname{dim}\left(L_{p}\right) \in \mathbb{Z}_{+}^{*}: p \in M\right\}$ o estrato $\Sigma_{k}$ será dito um estrato mínimo de $\underline{\mathcal{F}}$ e será denotado por $\Sigma_{\text {mín. }}$ Já quando $k=$ máx. $\left\{\operatorname{dim}\left(L_{p}\right): p \in M\right\}$ o estrato $\Sigma_{k}$ será dito um estrato regular de $\mathcal{F}$ e será denotado por $\Sigma_{\text {reg. }}$.

A partição $\left\{\Sigma_{k}\right\}_{k \in \mathbb{N}}$ de $M$ pelos seus estratos é de fato uma estratificação, no sentido apresentado em [13] e [3], como pode ser verificado na proprosição a seguir, cuja prova pode ser encontrada em [30] ou em [23], por exemplo.

\section{Proposição 2.7)}

a) $\quad \Sigma_{k} \subset M$ é uma subvariedade mergulhada conexa;

b) $\overline{\Sigma_{k}} \subset \cup_{j \leqslant k} \Sigma_{j}$, para todo $k \in \mathbb{N}$;

É imediato, da proposição anterior, que os estratos são subvariedades (mergulhadas) saturadas e, portanto, $\left(\Sigma_{k}, \mathcal{F}_{\Sigma_{k}}\right)$ é uma subvariedade folheada (regular). Além desta propriedade, nas proposições a seguir serão apresentadas algumas propriedades topológicas e métricas dos estratos de uma folheação.

\section{Proposição 2.8) (Propriedade métrica dos estratos) Seja}

$$
\Gamma_{k}:=\left\{\gamma \in C^{\infty}\left(\Sigma_{k}\right): \gamma \text { é um } g \text {-seg. de geod. horizontal e uma } g_{\Sigma_{k}} \text {-seg. de geod. }\right\}
$$

para cada $k \in \mathbb{N}$. Então $\Sigma_{k}=\cup_{\gamma \in \Gamma_{k}} \operatorname{Im} \gamma$, para todo $k \in \mathbb{N}$ e, em particular, $\left(\Sigma_{k}, g_{\Sigma_{k}}, \mathcal{F}_{\Sigma_{k}}\right)$ é uma folheação Riemanniana regular.

\section{Proposição 2.9) (Propriedades topológicas dos estratos)}

a) $\operatorname{codim}\left(\Sigma_{k}\right) \geq 2$, para todo $k<\operatorname{dim} \mathcal{F}$.

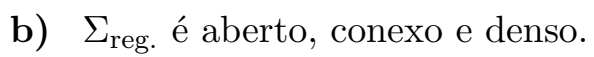




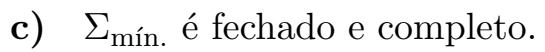

Proposição 2.10) Seja $L \in \mathcal{F}$ uma folha. Então $\bar{L} \subset M$ é uma subvariedade fechada, saturada e contida em um extrato.

\subsection{Homotetia, linearização de campos e folheação linearizada}

Sejam $(M, g)$ uma variedade Riemanniana, $\mathcal{F}$ uma FRS de $M, B \subset M$ uma subvariedade fechada, saturada e contida em um estrato (neste caso, $B$ é a união de folhas de mesma dimensão, e.g. o fecho de uma folha ou o estrato mínimo) e $W=\exp (\mathcal{W})$ uma VTN em torno de $B$, com projeção $\rho: W \longrightarrow B$.

\section{Homotetias}

Dado $\lambda \in \mathbb{R}$, será considerado $\mathrm{m}_{\lambda}: \nu B \longrightarrow \nu B$ o morfismo de fibrados vetoriais dado por $\mathrm{m}_{\lambda}(\eta):=\lambda \eta$. Quando $\lambda \in[0,1]$, tem-se que $\mathrm{m}_{\lambda}(\mathcal{W}) \subset \mathcal{W}$ e, portanto, $\left.\mathrm{m}_{\lambda}\right|_{\mathcal{W}}$ é um isomorfismo de fibrados (não necessariamente vetoriais) sobre a sua imagem, se $\lambda \neq 0$. Neste caso, para cada $\lambda \in[0,1]$, a aplicação $h_{\lambda}: W \longrightarrow W$ definida por

$$
h_{\lambda}:=\exp \circ \mathrm{m}_{\lambda} \circ\left(\left.\exp \right|_{\mathcal{W}}\right)^{-1}
$$

será dita uma $\lambda$-homotetia da folheação de $\mathcal{F}$ na $\operatorname{VTN} W$, de modo que $h_{\lambda}$ é um difeomorfismo sobre a sua imagem, se $\lambda \neq 0$. É imediato verificar que uma 0-homotetia é apenas a projeção $\rho$, ou seja, $h_{0}=\rho$ e uma 1-homotetia é apenas a identidade em $W$, ou seja, $h_{1}=\operatorname{Id}_{W}$.

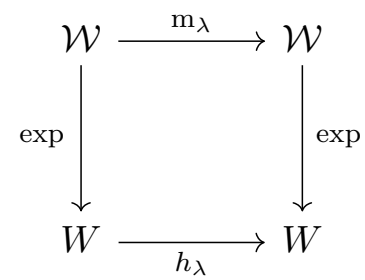

Cabe ressaltar que, dado $\eta \in \mathcal{W}$, a curva $t \longmapsto h_{t}(\exp (\eta))$ é um segmento da geodésica (radial) que passa por $\pi(\eta)$ com velocidade inicial $\eta$, ou seja, $\left.\frac{\mathrm{d}}{\mathrm{d} t} h_{t}(\exp (\eta))\right|_{t=0}=\eta$.

A seguir, serão apresentadas algumas propriedades a cerca da convergência das $\lambda$-homotetias quando $\lambda$ tende a zero. Isto será feito analisando-se a expressão das $\lambda$-homotetias em vizinhanças coordenadas de $W$.

Lema) (Convergência de homotetias) A sequência de homotetias $\left(h_{\lambda}\right)_{\lambda \in(0,1]}$ converge para $\rho$ na topologia $C^{\infty}$-compacto-aberta em $C^{\infty}(W, W)$, quando $\lambda$ tende a 0 . 
Demonstração. Sejam $\varepsilon>0,(\bar{\Phi}, U),(\bar{\Psi}, V)$ cartas trivializadoras de $W$ e $\bar{K} \subset W_{U}$ um compacto, tal que $\rho(\bar{K}) \subset V$.

Dado que exp $\left.\right|_{\mathcal{W}}$ é um isomorfismo de fibrados, $\left.\Phi \circ \exp \right|_{\mathcal{W}}$ e $\left.\Psi \circ \exp \right|_{\mathcal{W}}$ são cartas trivializadora de $\mathcal{W}$, que podem ser extendidas à cartas trivializadoras $\Phi: \nu B_{U} \longrightarrow \tilde{U} \times \mathbb{R}^{k} \subset \mathbb{R}^{n} \times \mathbb{R}^{k}$ e $\Psi: \nu B_{V} \longrightarrow \tilde{V} \times \mathbb{R}^{k} \subset \mathbb{R}^{n} \times \mathbb{R}^{k}$ (e.g. por homoteitas de $\nu B$ ).

Segue, para cada $\lambda \in(0,1]$, que

$$
\bar{\Psi} \circ h_{\lambda} \circ \bar{\Phi}^{-1}=\Psi \circ \mathrm{m}_{\lambda} \circ \Phi^{-1}, \quad \bar{\Psi} \circ \rho \circ \bar{\Phi}^{-1}=\Psi \circ \pi \circ \Phi^{-1},
$$

em $\bar{\Phi}\left(W_{U}\right)$. Mas

$$
\begin{aligned}
& \mathrm{D}^{r}\left(\Psi \circ \mathrm{m}_{\lambda} \circ \Phi^{-1}\right)(\tilde{x}, u)=\left(\mathrm{D}^{r}\left(\Psi_{n} \circ \Phi^{-1}\right)(\tilde{x}, u), \lambda \mathrm{D}^{r}\left(\Psi_{k} \circ \Phi^{-1}\right)(\tilde{x}, u)\right), \\
& \mathrm{D}^{r}\left(\Psi \circ \pi \circ \Phi^{-1}\right)(\tilde{x}, u)=\left(\mathrm{D}^{r}\left(\Psi_{n} \circ \Phi^{-1}\right)(\tilde{x}, u), 0\right),
\end{aligned}
$$

para todo $r \in \mathbb{Z}_{+}^{*}$ e $(\tilde{x}, u) \in \Phi\left(\nu B_{U}\right)$. Logo

$$
\left\|\mathrm{D}^{r}\left(\bar{\Psi} \circ h_{\lambda} \circ \bar{\Phi}^{-1}\right)(\tilde{x}, u)-\mathrm{D}^{r}\left(\bar{\Psi} \circ \rho \circ \bar{\Phi}^{-1}\right)(\tilde{x}, u)\right\|=\lambda\left\|\mathrm{D}^{r}\left(\Psi_{k} \circ \Phi^{-1}\right)(\tilde{x}, u)\right\|
$$

para todo $r \in \mathbb{Z}_{+}^{*}$ e $\bar{\Phi}\left(W_{U}\right)$.

Então, para cada $r \in \mathbb{Z}_{+}^{*}$, dado que $\bar{\Phi}(\bar{K})$ é compacto e $\mathrm{D}^{i}\left(\Psi_{k} \circ \Phi^{-1}\right)$ é contínua, para todo $i \in\{1, \cdots, r\}$, faz sentindo tomar $c_{r}=$ máx. $\left\{\left\|D^{i}\left(\Psi_{k} \circ \Phi^{-1}\right)(\tilde{x}, u)\right\|: i \in\{0, \cdots, r\},(\tilde{x}, u) \in\right.$ $\bar{\Phi}(\bar{K})\}$.

Segue, para cada $r \in \mathbb{Z}_{+}^{*}$, que

$$
\left\|\mathrm{D}^{i}\left(\bar{\Psi} \circ h_{\lambda} \circ \bar{\Phi}^{-1}\right)(\tilde{x}, u)-\mathrm{D}^{i}\left(\bar{\Psi} \circ \rho \circ \bar{\Phi}^{-1}\right)(\tilde{x}, u)\right\| \leqslant \lambda c_{r}<\varepsilon
$$

para todo $i \in\{0, \cdots, r\}, \lambda<\varepsilon c_{r}^{-1}$ e $(\tilde{x}, u) \in \bar{\Phi}(\bar{K})$.

Assim, $\left(h_{\lambda}\right)_{\lambda \in(0,1]}$ converge para $\rho$ quando $\lambda$ tende a 0 , na topologia $C^{r}$ - compacto-aberta, para todo $r \in \mathbb{Z}_{+}^{*}$, ou seja, converge na topologia $C^{\infty}$ - compacto-aberta em $C^{\infty}(W, W)$.

Observação) É possível adaptar a prova do lema anterior para demonstrar que $\lim _{\lambda \rightarrow \lambda_{0}} h_{\lambda}=h_{\lambda_{0}}$ na topologia $C^{\infty}$-compacto-aberta, para todo $\lambda_{0} \in[0,1]$, de modo que $\lambda \longmapsto h_{\lambda}$ é uma curva contínua em $C^{\infty}(W, W)$ com a topologia $C^{\infty}$-compacto-aberta.

Um dos resultados centrais na teoria de FRS é o Lema de homotetia (para demonstração do lema, ver [23] e [30], por exemplo). Em linhas gerais, este lema afirma que toda homotetia é folheada, fato que permitirá a criação do conceito de linearização em FRS, bem como permitirá transportar e extender os conceitos ligados a folheação para um ambiente mais "palatável", a saber, o fibrado normal a $B$.

Lema 2.11) (Lema de homotetia) Dado $\lambda \in[0,1]$ a homotetia $h_{\lambda}$ é folheada. Ainda, $h_{\lambda}$ é um difeomorfismo folheado (sobre a sua imagem), para todo $\lambda \in(0,1]$. 


\section{Linearização de campos}

Considere $\mathfrak{X}(W, B)$ o conjunto dos campos de $\mathfrak{X}(W)$, tais que $\left.X\right|_{B} \in \mathfrak{X}(B)$. A saber $\mathfrak{X}(W, B) \subset$ $\mathfrak{X}(W)$ é uma subálgebra de Lie e um $C^{\infty}(W)$-submódulo.

Ainda, para cada $\lambda \in(0,1]$ e $X \in \mathfrak{X}(W, B)$, será considerado $X_{\lambda} \in \mathfrak{X}(W)$ dado por

$$
X_{\lambda}:=\left(h_{\lambda}\right)^{*} X=\mathrm{d} h_{\lambda}^{-1} \circ X \circ h_{\lambda} .
$$

A partir dos campos de $\mathfrak{X}(W, B)$ será definida, a seguir, a noção de linearização de campos que a princípio independe do Lema de homotetia. Contudo, tal lema irá garantir boas propriedades aos campos linearizados, como será visto mais a frente nas Proposições 2.14 e 2.15 .

Proposição 2.12) (Campo linearizado) Seja $X \in \mathfrak{X}(W, B)$. Então $\left(X_{\lambda}\right)_{\lambda \in(0,1]}$ converge em $\mathfrak{X}(W)$ na topologia $C^{\infty}$-compacto-aberta, quando $\lambda$ tende 0 .

Demonstração. Inicialmente, será contruída uma carta de $W$, com boas propriedades, para em seguida, ao obter a expressão dos campos $X_{\lambda}$ nesta carta, concluir a convergência dos mesmos.

Seja $\tilde{\varphi}: \tilde{U} \subset B \longrightarrow \mathbb{R}^{n}$ uma carta de $B$, com $\tilde{\varphi}(\tilde{U})=U$, e $\tilde{\Phi}: \nu B_{\tilde{U}} \longrightarrow \tilde{U} \times \mathbb{R}^{k}$ uma trivialização de $\nu B$. Observe que $(\tilde{\varphi} \times \mathrm{Id}) \circ \tilde{\Phi}$ é uma carta de $\nu B$.

Segue que $\Phi: W_{\tilde{U}} \longrightarrow \mathbb{R}^{n} \times \mathbb{R}^{k}$ dada por $\Phi:=\left.((\tilde{\varphi} \times \mathrm{Id}) \circ \tilde{\Phi})\right|_{\mathcal{W}} \circ(\exp \mid \mathcal{W})^{-1}$ é uma carta de $W_{U}$, tal que

$$
\left(\Phi \circ h_{\lambda} \circ \Phi^{-1}\right)(u, x)=(u, \lambda x)
$$

para todo $\lambda \in[0,1]$ e $(u, x) \in \Phi\left(W_{U}\right)$.

Logo

$$
\left(\Phi \circ h_{\lambda} \circ \Phi^{-1}\right)_{*} e_{i}=e_{i}, \quad\left(\Phi \circ h_{\lambda} \circ \Phi^{-1}\right)_{*} e_{n+j}=\lambda e_{n+j},
$$

para todo $i \in\{1, \cdots, n\}$ e $j \in\{1, \cdots, k\}$, onde $\left\{e_{1}, \cdots, e_{n+k}\right\}$ é a base canônica de $\mathbb{R}^{n+k}$ e, considerando-se

$$
\Phi_{*} X=\sum_{i=1}^{n} b_{i} e_{i}+\sum_{j=1}^{k} c_{j} e_{n+j}
$$

tem-se que

$$
\left.\Phi_{*} X_{\lambda}\right|_{(u, x)}=\left.\left(\Phi \circ h_{\lambda^{-1}} \circ \Phi^{-1}\right)_{*} \Phi_{*} X\right|_{(u, x)}=\sum_{i=1}^{n} b_{i}(u, \lambda x) e_{i}+\sum_{j=1}^{k} c_{j}(u, \lambda x) \frac{1}{\lambda} e_{n+j}
$$

Assim $\left(X_{\lambda}\right)_{\lambda \in(0,1]}$ converge em $\mathfrak{X}(W)$ na topologia $C^{\infty}$ - compacto-aberta. 
O campo vetorial $X^{\ell} \in \mathfrak{X}(W)$ dado por

$$
X^{\ell}:=\lim _{\lambda \rightarrow 0} X_{\lambda}
$$

será dito a linearização do campo vetorial $X$ na VTN $W$ e o conjunto dos campos linearizados será denotado por $\mathfrak{X}^{\ell}(W)$, ou seja,

$$
\mathfrak{X}^{\ell}(W):=\left\{X^{\ell} \in \mathfrak{X}(W): X \in \mathfrak{X}(W, B)\right\}
$$

A razão do uso do termo "linearizado", empregado para nomear os campos $X^{\ell}$, ficará mais clara no item (a) da Proposição 2.17.

A seguir será apresentada uma sequência extenuante de propriedades dos campos linearizados (Lema 2.13, Proposição 2.14 e Proposição 2.15) e de seus fluxos, que serão importantes para a compreensão futura do texto.

Lema 2.13) (Propriedades algébricas dos campos linearizados) Considere $C_{\rho}^{\infty}(W)$ o anel das funções $\rho$-básicas, ou seja, $C_{\rho}^{\infty}(W):=\left\{f \in C^{\infty}(W): f \circ \rho\right.$ é constante $\}$. Então

a) $(f X)^{\ell}=(f \circ \rho) X^{\ell}$, para todo $f \in C^{\infty}(W)$ e $X \in \mathfrak{X}(W, B)$. Em particular, $\mathfrak{X}^{\ell}(W) \subset$ $\mathfrak{X}(W)$ é um $C_{\rho}^{\infty}(W)$ - submódulo.

b) $[X, Y]^{\ell}=\left[X^{\ell}, Y^{\ell}\right]$, para todo $X, Y \in \mathfrak{X}(W, B)$. Em particular, $\mathfrak{X}^{\ell}(W) \subset \mathfrak{X}(W)$ é uma subálgebra de Lie.

c) A aplicação $\theta: \mathfrak{X}(W, B) \longrightarrow \mathfrak{X}^{\ell}(W)$ dada por $\theta(X):=X^{\ell}$ é um morfismo sobrejetor de álgebras de Lie e de $C_{\rho}^{\infty}(W)$-bmódulos.

Demonstração. É imediato da definição de campo linearizado que $(X+Y)^{\ell}=X^{\ell}+Y^{\ell}$, para todo $X, Y \in \mathfrak{X}(W, B)$.

a) Dados $f \in C^{\infty}(W)$ e $X \in \mathfrak{X}(W, B)$, tem-se que

$$
\left.(f X)^{\ell}\right|_{p}=\left.\lim _{\lambda \rightarrow 0} f\left(h_{\lambda}(p)\right) \mathrm{d} h_{\lambda}^{-1}\right|_{h_{\lambda}(p)}\left(\left.X\right|_{h_{\lambda}(p)}\right)=\left.f(\rho(p)) X^{\ell}\right|_{p}
$$

para todo $p \in W$.

Assim $(f X)^{\ell}=(f \circ \rho) X^{\ell}$, para todo $f \in C^{\infty}(W)$ e $X \in \mathfrak{X}(W, B)$.

b) $\operatorname{Dados} X, Y \in \mathfrak{X}(W, B)$, tem-se que $[X, Y] \in \mathfrak{X}(W, B)$ e

$$
\left[X_{\lambda}, Y_{\lambda}\right]=\left[\left(h_{\lambda}\right)^{*} X,\left(h_{\lambda}\right)^{*} Y\right]=\left(h_{\lambda}\right)^{*}[X, Y]=[X, Y]_{\lambda}
$$

para todo $\lambda \in(0,1]$.

Assim, como o colchete de Lie é contínuo na topologia $C^{\infty}$ - compacto-aberta, conclui-se que

$$
[X, Y]^{\ell}=\lim _{\lambda \rightarrow 0}[X, Y]_{\lambda}=\left[\lim _{\lambda \rightarrow 0} X_{\lambda}, \lim _{\lambda \rightarrow 0} Y_{\lambda}\right]=\left[X^{\ell}, Y^{\ell}\right]
$$


c) Segue diretamente dos itens anteriores.

Proposição 2.14) (Propriedades dos campos linearizados) Seja $X \in \mathfrak{X}(W, B)$. Então

a) $\left.X^{\ell}\right|_{B}=\left.X\right|_{B}$.

b) $X^{\ell}$ é $h_{\lambda}$-invariante, para todo $\lambda \in(0,1]$.

c) $\quad X^{\ell}$ é $\rho$-invariante ( $\rho$-projetável).

d) $\left(X^{\ell}\right)^{\ell}=X^{\ell}$.

e) Se $X \in \mathfrak{X}\left(\mathcal{F}_{W}\right)$, então $X^{\ell} \in \mathfrak{X}\left(\mathcal{F}_{W}\right)$.

f) Se $X$ é $\mathcal{F}_{W}$-folheado, então $X^{\ell}$ é $\mathcal{F}_{W}$-folheado.

\section{Demonstração.}

a) Seja $b \in B$. Dados $\lambda \in[0,1]$ e $u \in \mathrm{T}_{b} B$ e considerando-se $\gamma$ uma curva em $B$, tal que $\gamma(0)=b$ e $\gamma^{\prime}(0)=u$, tem-se que

$$
\left.\mathrm{d} h_{\lambda}\right|_{b}(u)=\left.\frac{\mathrm{d}}{\mathrm{d} t} h_{\lambda}(\gamma(t))\right|_{t=0}=\left.\frac{\mathrm{d}}{\mathrm{d} t} \exp \left(\mathrm{m}_{\lambda}\left(\left.\exp \right|_{\mathcal{W}} ^{-1}(\gamma(t))\right)\right)\right|_{t=0}=\gamma^{\prime}(0)=u
$$

Logo $\left.\mathrm{d}_{\lambda}^{-1}\right|_{h_{\lambda}(b)}(u)=u$, para todo $\lambda \in[0,1]$ e $u \in \mathrm{T}_{b} B$.

Assim

$$
\left.X^{\ell}\right|_{b}=\left.\lim _{\lambda \rightarrow 0} \mathrm{~d} h_{\lambda}^{-1}\right|_{h_{\lambda}(b)}\left(\left.X\right|_{h_{\lambda}(b)}\right)=\left.X\right|_{\lim _{\lambda \rightarrow 0} h_{\lambda}(b)}=\left.X\right|_{b}
$$

b) Segue que

$$
\begin{aligned}
\left.\mathrm{d} h_{\mu}\right|_{p}\left(\left.X^{\ell}\right|_{p}\right) & =\left.\mathrm{d} h_{\mu}\right|_{p}\left(\left.\lim _{\lambda \rightarrow 0} \mathrm{~d} h_{\lambda}^{-1}\right|_{h_{\lambda}(p)}\left(\left.X\right|_{h_{\lambda}(p)}\right)\right) \\
& =\lim _{\lambda \rightarrow 0}\left(\left.\mathrm{~d} h_{\mu \lambda^{-1}}\right|_{h_{\lambda}(p)}\left(\left.X\right|_{h_{\lambda}(p)}\right)\right) \\
& =\lim _{\mu \lambda^{-1} \rightarrow 0}\left(\left.\mathrm{~d} h_{\left(\lambda \mu^{-1}\right)^{-1}}\right|_{h_{\lambda \mu^{-1}}\left(h_{\mu}(p)\right)}\left(\left.X\right|_{h_{\lambda \mu^{-1}}\left(h_{\mu}(p)\right)}\right)\right) \\
& =\left.X^{\ell}\right|_{h_{\mu}(p)},
\end{aligned}
$$

para todo $\mu \in(0,1]$.

c) Uma vez que $X^{\ell}$ é invariante por homotetias e a diferencial de funções é uma operação contínua na topologia $C^{\infty}$ - compacto-aberta, segue que

$$
\left.\mathrm{d} \rho\right|_{q}\left(\left.X^{\ell}\right|_{q}\right)=\left.\lim _{\lambda \rightarrow 0} \mathrm{~d} h_{\lambda}\right|_{q}\left(\left.X^{\ell}\right|_{q}\right)=\left.\lim _{\lambda \rightarrow 0} X^{\ell}\right|_{h_{\lambda}(q)}=\left.X^{\ell}\right|_{\lambda \rightarrow 0} h_{\lambda(q)}=\left.X^{\ell}\right|_{\rho(q)},
$$

para todo $q \in W$. 
d) Segue, da invariância por homotetias do campo $X^{\ell}$, que

$$
\left.\left(X^{\ell}\right)^{\ell}\right|_{p}=\left.\lim _{\lambda \rightarrow 0} \mathrm{~d} h_{\lambda}^{-1}\right|_{h_{\lambda}(p)}\left(\left.X^{\ell}\right|_{h_{\lambda}(p)}\right)=\left.\lim _{\lambda \rightarrow 0} X^{\ell}\right|_{p}=\left.X^{\ell}\right|_{p}
$$

para todo $p \in W$.

e) Uma vez que $X \in \mathfrak{X}\left(\mathcal{F}_{W}\right)$ e $h_{\lambda}$ é folheado, segue que

$$
\left.X^{\ell}\right|_{p}=\left.\lim _{\lambda \rightarrow 0} \mathrm{~d} h_{\lambda}^{-1}\right|_{h_{\lambda}(p)}\left(\left.X\right|_{h_{\lambda}(p)}\right) \in \mathrm{T}_{p} L_{p}
$$

para todo $p \in W$.

$\operatorname{Assim} X^{\ell} \in \mathfrak{X}\left(\mathcal{F}_{W}\right)$.

f) Dado $V \in \mathfrak{X}\left(\mathcal{F}_{W}\right)$, pelo item anterior, tem-se que $V^{\ell} \in \mathfrak{X}\left(\mathcal{F}_{W}\right)$.

Uma vez que $X$ é $\mathcal{F}_{W}$-folheado, segue que $\left[X, V^{\ell}\right] \in \mathfrak{X}\left(\mathcal{F}_{W}\right)$ e, portanto,

$$
\left[X^{\ell}, V\right]=\left[X^{\ell},\left(V^{\ell}\right)^{\ell}\right]=\left[X, V^{\ell}\right]^{\ell} \in \mathfrak{X}\left(\mathcal{F}_{W}\right) .
$$

Assim $\left[X^{\ell}, V\right] \in \mathfrak{X}\left(\mathcal{F}_{W}\right)$, para todo $V \in \mathfrak{X}\left(\mathcal{F}_{W}\right)$, ou seja, $X^{\ell} \in \mathfrak{X}\left(\mathcal{F}_{W}\right)$.

Segue, das propriedades exibidas, que o conjunto das linearizações dos campos tangentes à folheação

$$
\mathfrak{X}\left(\mathcal{F}_{W}\right)^{\ell}:=\left\{X^{\ell} \in \mathfrak{X}(W): X \in \mathfrak{X}\left(\mathcal{F}_{W}\right)\right\}
$$

é uma subálgebra de Lie de $\mathfrak{X}^{\ell}\left(\mathcal{F}_{W}\right):=\mathfrak{X}\left(\mathcal{F}_{W}\right) \cap \mathfrak{X}^{\ell}(W)$. Assim como $\mathfrak{X}^{\ell}(W)$, os conjuntos $\mathfrak{X}^{\ell}\left(\mathcal{F}_{W}\right)$ e $\mathfrak{X}\left(\mathcal{F}_{W}\right)^{\ell}$ não são $C^{\infty}(W)$-submódulos de $\mathfrak{X}(W)$, mas são $C_{\rho}^{\infty}(W)$-submódulos de $\mathfrak{X}^{\ell}(W)$.

Proposição 2.15) (Propriedades do fluxo de campos linearizados) Seja $X \in \mathfrak{X}(W, B)$ e considere, para cada $t \in \mathbb{R}, \phi_{t}: D_{t} \subset W \longrightarrow W$ o fluxo de $X^{\ell}$ no instante $t$. Então

a) $D_{t} \cap W_{b}=W_{b}$ e $\phi_{t}\left(W_{b}\right) \subset W_{\phi_{t}(b)}$, para todo $b \in \rho\left(D_{t}\right)$. Em particular, $D_{t}=W_{\rho\left(D_{t}\right)}$,

b) $\phi_{t} \circ \exp =\exp \circ \mathrm{d} \phi_{t}$, em $\mathcal{W}$,

para todo $t \in \mathbb{R}$, tal que $D_{t} \neq \emptyset$.

Ainda, se $X \in \mathfrak{X}(\mathcal{F})$, então

c) $\left.\phi_{t}\right|_{W_{b}}$ é uma isometria, para todo $b \in \rho\left(D_{t}\right)$,

d) $\mathrm{d} \phi_{t} \circ Y^{\ell}=\left(\left(\phi_{t}\right)_{*} Y\right)^{\ell} \circ \phi_{t}$, em $D_{t}$, para todo $Y \in \mathfrak{X}\left(\mathcal{F}_{W}\right)$,

para todo $t \in \mathbb{R}$, tal que $D_{t} \neq \emptyset$.

Demonstração. Dado $p \in W$ será considerado $D_{p}=\{q \in W:(t, q) \in D\}$. 
a) Seja $b \in \rho\left(D_{t}\right)$, de modo que $W_{b} \cap D_{t} \neq \emptyset$, e considere $p \in W_{b} \cap D_{t}$.

Segue, da $\rho$-invariância de $X^{\ell}$, que $D_{p} \subset D_{\rho(p)}=D_{b}$ e, como $t \in D_{q}$, tem-se que $b \in W_{b} \cap D_{t}$, ou seja, $W_{b} \cap D_{t} \subset W_{b}$ é um aberto em torno de $b$.

Logo, dado $\tilde{p} \in W_{b}$, existe $\lambda \in(0,1]$ e $\tilde{q} \in W_{b} \cap D_{t}$, tal que $\tilde{p}=h_{\lambda}(\tilde{q})$. Segue, da $h_{\lambda^{-}}$ invariância de $X^{\ell}$, que $D_{\tilde{q}} \subset D_{h_{\lambda}(\tilde{q})}=D_{\tilde{p}}$ e, como $t \in D_{\tilde{q}}$, tem-se que $\tilde{p} \in W_{b} \cap D_{t}$.

Assim $W_{b} \subset W_{b} \cap D_{t}$, ou seja, $W_{b}=W_{b} \cap D_{t}$.

Ainda, como $X^{\ell}$ é $\pi$-invariante, dado $b \in \pi\left(D_{t}\right)$, segue que $\pi\left(\phi_{t}(p)\right)=\phi_{t}(\pi(p))=\phi_{t}(b)$, para todo $p \in W_{b}$.

Assim $\phi_{t}\left(W_{b}\right) \subset W_{\phi_{t}(b)}$, para todo $b \in \pi\left(D_{t}\right)$.

b) Uma vez que $X^{\ell}$ é $\pi$-invariante e invariante por homotetias, tem-se que $\phi_{t} \circ h_{s}=h_{s} \circ \phi_{t}$, para todo $s \in[0,1]$ e $t \in \mathbb{R}$, tal que $D_{t} \neq \emptyset$ e, portanto,

$$
\begin{aligned}
\left.\mathrm{d} \phi_{t}\right|_{b}(\eta) & =\left.\mathrm{d} \phi_{t}\right|_{h_{0}(\exp (\eta))}\left(\left.\frac{\mathrm{d}}{\mathrm{d} s} h_{s}(\exp (\eta))\right|_{s=0}\right) \\
& =\left.\frac{\mathrm{d}}{\mathrm{d} s}\left(\phi_{t} \circ h_{s}\right)(\exp (\eta))\right|_{s=0} \\
& =\left.\frac{\mathrm{d}}{\mathrm{d} s}\left(h_{s} \circ \phi_{t}\right)(\exp (\eta))\right|_{s=0} \\
& =\exp ^{-1}\left(\phi_{t}(\exp (\eta))\right),
\end{aligned}
$$

para todo $\eta \in \nu^{\varepsilon} B$, com $\pi_{\nu B}(\eta)=b$ e $\exp (\eta)=p$.

Assim $\phi_{t} \circ \exp =\exp \circ \mathrm{d} \phi_{t}, \operatorname{em} \mathcal{W}$.

c) Segue do item anterior e do fato de que a folheação é localmente equidistante (ver Proposição 2.5) que $\left.\phi_{t}\right|_{W_{b}}$ é uma isometria.

d) Observe, primeiramente, que $D_{t}=W_{\rho\left(D_{t}\right)}$ (ver item (a)), ou seja, $D_{t}$ é uma $\varepsilon$-VTD em torno do aberto $\rho\left(D_{t}\right) \subset B$ e, portanto, é possível considerar a linearização de $\left(\phi_{t}\right)_{*} Y$ em torno de $\rho\left(D_{t}\right)$ na vizinhança $D_{t}$.

Segue, da definição de linearização de campos, que

$$
\begin{aligned}
\left.\mathrm{d} \phi_{t}\right|_{x}\left(\left.Y^{\ell}\right|_{x}\right) & =\left.\mathrm{d} \phi_{t}\right|_{x}\left(\left.\lim _{\lambda \rightarrow 0} \mathrm{~d} h_{\lambda}^{-1}\right|_{h_{\lambda}(x)}\left(\left.Y\right|_{h_{\lambda}(x)}\right)\right) \\
& =\left.\left.\lim _{\lambda \rightarrow 0} \mathrm{~d}\left(\phi_{t} \circ h_{\lambda}^{-1}\right)\right|_{h_{\lambda}(x)} Y\right|_{h_{\lambda}(x)} \\
& =\left.\left.\lim _{\lambda \rightarrow 0} \mathrm{~d}\left(h_{\lambda}^{-1} \circ \phi_{t}\right)\right|_{h_{\lambda}(x)} Y\right|_{h_{\lambda}(x)} \\
& =\left.\lim _{\lambda \rightarrow 0} \mathrm{~d} h_{\lambda}^{-1}\right|_{\phi_{t}\left(h_{\lambda}(x)\right)}\left(\left.\mathrm{d} \phi_{t}\right|_{h_{\lambda}(x)}\left(\left.Y\right|_{h_{\lambda}(x)}\right)\right),
\end{aligned}
$$

para todo $x \in D_{t}$. 
Mas

$$
\left.\left(\phi_{t}\right)_{*} Y\right|_{h_{\lambda}\left(\phi_{t}(x)\right)}=\left.\mathrm{d} \phi_{t}\right|_{\phi_{t}^{-1}\left(h_{\lambda}\left(\phi_{t}(x)\right)\right.}=\left.\mathrm{d} \phi_{t}\right|_{h_{\lambda}(x)}\left(\left.Y\right|_{h_{\lambda}(x)}\right),
$$

para todo $x \in D_{t}$.

Assim

$$
\left.\mathrm{d} \phi_{t}\right|_{x}\left(\left.Y^{\ell}\right|_{x}\right)=\left.\lim _{\lambda \rightarrow 0} \mathrm{~d} h_{\lambda}^{-1}\right|_{h_{\lambda}\left(\phi_{t}(x)\right)}\left(\mathrm{d} \phi_{t} \mid\left(\left.Y\right|_{h_{\lambda}(x)}\right)\right)
$$

para todo $x \in D_{t}$, ou seja, $\mathrm{d} \phi_{t} \circ Y^{\ell}=\left(\left(\phi_{t}\right)_{*} Y\right)^{\ell} \circ \phi_{t}\left(\right.$ em $\left.D_{t}\right)$.

\section{Linearização de distribuições}

Dado $H \subset \mathrm{T} W$ uma distribuição (singular) suave, tal que $\left.H\right|_{B} \subset \mathrm{T} B$, considere $\mathfrak{X}(H)$ o conjunto dos campos vetoriais de $W$ tangentes a $H$ de modo que $\mathfrak{X}(H) \subset \mathfrak{X}(W, B)$ o que permite considerar a linearização dos campos de $\mathfrak{X}(H)$.

Assim, é possível definir a distribuição (singular) suave $H^{\ell} \subset \mathrm{T} W$ por $H^{\ell}:=\operatorname{span}\left\{X^{\ell} \in\right.$ $\left.\mathfrak{X}(W)^{\ell}: X \in \mathfrak{X}(H)\right\}$, ou seja,

$$
\left.H^{\ell}\right|_{p}=\operatorname{span}\left\{\left.X^{\ell}\right|_{p} \in \mathrm{T}_{p} W: X \in \mathfrak{X}(H)\right\},
$$

para todo $p \in W$, a qual será dita a linerização da distribuição $H$ na VTN W.

Lema) Se $H$ é regular, então $H^{\ell}$ é regular, $\operatorname{com} \operatorname{posto}\left(H^{\ell}\right)=\operatorname{posto}(H)$.

Demonstração. Considere posto $(H)=k$ e seja $p \in W$.

Suponha que $\operatorname{dim}\left(\left.H^{\ell}\right|_{p}\right)>k$. Neste caso, existem campos $X_{1}, \cdots, X_{k+1} \in \mathfrak{X}(H)$, tais que $\left\{\left.X_{1}^{\ell}\right|_{p}, \cdots,\left.X_{k+1}^{\ell}\right|_{p}\right\}$ é linearmente independente.

Mas $\left\{X_{1}, \cdots, X_{k+1}\right\}$ é linearmente dependente em $W$ e, portanto, $X_{k+1}=\sum_{i=1}^{k} f_{i} X_{k}$, com $f_{i}: W \longrightarrow \mathbb{R}$ suave.

Logo $X_{k+1}^{\ell}=\sum_{i=1}^{k}\left(f_{i} \circ \rho\right) X_{k}^{\ell}$ e, em particular, $\left.X_{k+1}^{\ell}\right|_{p}=\left.\sum_{i=1}^{k} f_{i}(\rho(p)) X_{k}^{\ell}\right|_{p}$ (absurdo !).

Assim, dado que $\left.H^{\ell}\right|_{B}=\left.H\right|_{B}$, conclui-se que

$$
k=\operatorname{posto}(H)=\operatorname{dim}\left(\left.H^{\ell}\right|_{\rho(p)}\right) \leqslant \operatorname{dim}\left(\left.H^{\ell}\right|_{p}\right) \leqslant k,
$$

ou seja, $\operatorname{dim}\left(\left.H^{\ell}\right|_{p}\right)=\operatorname{posto}(H)$.

Devido a linearização de distribuições ser definida por meio do conceito de linearização de campos, as propriedades dos campos linearizados expressas na Proposição 2.14, valem de modo similar à linearização de distribuições, como será apresentado na lema seguir.

Lema) (Propriedades da linearizacao de distribuições) Seja $H \subset \mathrm{T} W$ uma distribuição 
(singular) suave, tal que $\left.H\right|_{B} \subset \mathrm{T} B$. Então

a) $H^{\ell}$ é $h_{\lambda}$-invariante, para todo $\lambda \in(0,1]$.

b) $H^{\ell}$ é $\rho$-invariante (projetável) e, em particular, $\left.H^{\ell}\right|_{B}=\left.H\right|_{B}$.

c) $\left(H^{\ell}\right)^{\ell}=H^{\ell}$,

d) Se $H$ é tangente a folheação $\mathcal{F}_{W}$, então $H^{\ell}$ é tangente a folheação $\mathcal{F}_{W}$.

\section{Folheação linearizada}

Uma consequência imediata do item (d) da Proposição 2.15 é o fato de que a distribuição $\operatorname{span}\left(\mathfrak{X}\left(\mathcal{F}_{W}\right)^{\ell}\right)$ é $\mathfrak{X}\left(\mathcal{F}_{W}\right)^{\ell}$ - invariante (conceito definido na Seção 1.2), de modo que aplicando-se o Teorema de Sussmann à subálgebra de Lie $\mathfrak{X}\left(\mathcal{F}_{W}\right)^{\ell} \subset \mathfrak{X}(W)$ (mais precisamente o Corolário 1.13, decorrente de tal teorema), obtém-se uma folheção singular de $W$, cuja folha pasando por $p \in W$ é dada por

$$
L_{p}^{\ell}:=\left\{\phi_{t}^{X^{\ell}}(p) \in W: n \in \mathbb{Z}_{+}^{*}, X \in \mathfrak{X}\left(\mathcal{F}_{W}\right)^{n}, t \in \mathbb{R}^{n}: p \in D_{t}^{X^{\ell}}\right\},
$$

onde $\phi_{t}^{X^{\ell}}: D_{t}^{X^{\ell}} \subset \mathbb{R} \times W \longrightarrow W$ é a composta dos fluxos de $X_{i}^{\ell}$ nos instantes $t_{i}$, ou seja, $\phi_{t}^{X^{\ell}}=\phi_{t_{n}}^{X_{n}^{\ell}} \circ \cdots \circ \phi_{t_{1}}^{X_{1}^{\ell}} ;$ e o plano tangente a esta folha é dado por

$$
\mathrm{T}_{p} L_{p}^{\ell}=\left\{\left.X^{\ell}\right|_{p} \in \mathrm{T}_{p} W: X \in \mathfrak{X}\left(\mathcal{F}_{W}\right)\right\}
$$

A folheação singular, assim obtida, será dita a folheação linearizada ou a linearização de $\mathcal{F}$ na VTN $W$ e será denotada por $\mathcal{F}^{\ell}$. Cabe ressaltar que, como consequência imediata da definição de $\mathcal{F}^{\ell}$, valem para esta folheação propriedades análogas as propriedades dos campos linearizados, apresantadas na Proposição 2.14.

\section{Lema) (Propriedades da folheação linearizada)}

a) $B \subset M$ é uma subvariedade $\mathcal{F}^{\ell}$-saturada e contida em um $\mathcal{F}^{\ell}$-estrato, de modo que $\left(\mathcal{F}^{\ell}\right)_{B}=\mathcal{F}_{B}$

b) $h_{\lambda}$ é $\mathcal{F}^{\ell}$ - folheada, para todo $\lambda \in(0,1]$.

c) $\rho:\left(W, \mathcal{F}^{\ell}\right) \longrightarrow\left(B, \mathcal{F}_{B}^{\ell}\right)$ é folheada.

d) $\left(\mathcal{F}^{\ell}\right)^{\ell}=\mathcal{F}^{\ell}$.

e) $\mathcal{F}^{\ell}<\mathcal{F}_{W}$.

Observação) A princípio, o item (d) do lema anterior parece não fazer sentido, dado que o conceito de linearização de folheações foi apresentado somente para o caso de FRS.

Entretanto, o argumento que constrói a folheação linearizada pode ser aplicado a qualquer folheação singular (não necessariamente Riemanniana) de uma VTN, que seja invariante por homotetias. Precisamente, este é o caso da folheação linearizada $\mathcal{F}^{\ell}$, vide item (b) do lema anterior. 


\section{Extensões homotéticas ao fibrado normal}

Esta seção se encerrará com a apresentação de uma consequência importante do Lema de homotetia. De modo geral, objetos invariantes por homotetia em uma VTN $W$ podem ser identificados com objetos invariantes por homotetias no aberto $\mathcal{W}$ do fibrado normal a $B$. Neste ambiente, tais objetos podem ser extendidos por meio da homotetia "natural" do fibrado normal, a saber, $\mathrm{m}_{\lambda}: \nu B \longrightarrow \nu B$ dada por $\mathrm{m}_{\lambda}(\eta)=\lambda \eta$. Entre tais objetos, devido ao Lema de homotetia, encontram-se as folheações $\mathcal{F}_{W}$ e $\mathcal{F}^{\ell}$, que serão identificadas com folheações no aberto $\mathcal{W}$ e extendida à folheações no fibrado normal a $B$. Assim o estudo da dinâmica da folheação em uma VTN é similar ao estudo de uma folheação em um fibrado vetorial, com propriedades apropriadas.

Lema) (Extensão homotética de campos) Seja $X \in \mathfrak{X}(W)$ invariante por $\lambda$-homotetias, para todo $\lambda \in(0,1]$. Então, existe um único campo $X^{\nu} \in \mathfrak{X}(\nu B)$ invariante por homotetias, tal que $\left.X^{\nu}\right|_{\mathcal{W}}=\left(\left.\exp \right|_{\mathcal{W}}\right)^{*} X$

Demonstração. Uma vez que $\left.h_{\lambda} \circ \exp \right|_{\mathcal{W}}=\exp \circ \mathrm{m}_{\lambda} \mid \mathcal{W}$, segue que $\left(\left.\exp \right|_{\mathcal{W}}\right)^{*} X$ é um campo suave invariante por homotetias em $\mathcal{W}$.

Defina $X^{\nu} \in \mathfrak{X}(\nu B)$ por

$$
\left.X^{\nu}\right|_{\eta}:=\left.\mathrm{dm}_{\lambda}\right|_{\eta} ^{-1}\left(\left.X\right|_{\lambda \eta}\right)
$$

com $\lambda \in \mathbb{R}$, tal que $\lambda \eta \in \mathcal{W}$. Observe que $X^{\nu}$ está bem definido, pois se $\mu \in \mathbb{R}$ é tal que $\mu \eta \in \mathcal{W}$, supondo-se sem perda de generalidade que $\mu<\lambda$, tem-se que

$$
\begin{aligned}
\left.\operatorname{dm}_{\lambda}\right|_{\eta} ^{-1}\left(\left.X\right|_{\lambda \eta}\right) & =\left.\operatorname{dm}_{\mu}\right|_{\eta} ^{-1}\left(\left.\operatorname{dm}_{\mu \lambda^{-1}}\right|_{\lambda \eta} ^{-1}\left(\left.X\right|_{\lambda \eta}\right)\right) \\
& =\left.\operatorname{dm}_{\mu}\right|_{\eta} ^{-1}\left(\left.X\right|_{\mathrm{m}_{\mu \lambda-1}(\lambda \eta)}\right) \\
& =\left.\operatorname{dm}_{\mu}\right|_{\eta} ^{-1}\left(\left.X\right|_{\mu \eta}\right) .
\end{aligned}
$$

A invariância por homotetias de $X^{\nu}$ segue diretamente da definição e a unicidade de $X^{\nu}$ segue da invariância por homotetias.

Lema) (Extensão homotética de folheações) Seja $\mathcal{F}$ uma folheação de $W$ invariante por $\lambda$-homotetias, para todo $\lambda \in(0,1]$. Então, existe uma única folheação $\mathcal{F}^{\nu}$ de $\nu B$, tal que $\left.\mathcal{F}^{\nu}\right|_{\mathcal{W}}=(\exp \mid \mathcal{W})^{*} \mathcal{F}_{W}$

Demonstração. Uma vez que $\left.h_{\lambda} \circ \exp \right|_{\mathcal{W}}=\left.\exp \circ \mathrm{m}_{\lambda}\right|_{\mathcal{W}}$, pelo Lema de homotetia, segue que $(\exp \mid \mathcal{W})^{*} \mathcal{F}_{W}$ é uma folheação invariante por $\lambda$-homotetias em $\mathcal{W}$, para todo $\lambda \in(0,1]$.

Defina, para cada $\eta \in \nu B$,

$$
L_{\eta}^{\nu}=\mathrm{m}_{\lambda}^{-1}\left(L_{\lambda \eta}\right)
$$

com $\lambda \in \mathbb{R}$, tal que $\lambda \eta \in \mathcal{W}$, onde $L_{\lambda \eta}$ é a folha de $(\exp \mid \mathcal{W})^{*} \mathcal{F}$ passando por $\lambda \eta$. A invariância por homotetias da folheação $(\exp \mid \mathcal{W})^{*} \mathcal{F}_{W}$, garante que $L_{\eta}^{\nu}$ está bem definida, pois se $\mu \in \mathbb{R}$ é 
tal que $\mu \eta \in \mathcal{W}$, supondo-se em perda de generalidade que $\mu<\lambda$, tem-se que

$$
\mathrm{m}_{\lambda}^{-1}\left(L_{\lambda \eta}\right)=\mathrm{m}_{\mu}^{-1}\left(\mathrm{~m}_{\mu \lambda^{-1}}\left(L_{\lambda \eta}\right)\right)=\mathrm{m}_{\mu}^{-1}\left(L_{\mu \eta}\right)
$$

Dado que $\mathrm{m}_{\lambda}$ é uma isomorfismo, para todo $\lambda \in \mathbb{R}^{*}$, a partição $\mathcal{F}^{\nu}:=\left\{L_{\eta}^{\nu}\right\}_{\eta \in \nu B}$ é dada por subvariedades imersas de $\nu B$. Ainda, para cada $X \in \mathfrak{X}\left(\left(\left.\exp \right|_{\mathcal{W}}\right)^{*} \mathcal{F}_{W}\right)$, segue do lema anterior, que $X^{\nu} \in \mathfrak{X}\left(\mathcal{F}^{\nu}\right)$.

Assim $\mathcal{F}^{\nu}$ é uma folheação singular de $\nu B$ invariante por homotetias, tal que $\left.\mathcal{F}^{\nu}\right|_{\mathcal{W}}=$ $(\exp \mid \mathcal{W})^{*} \mathcal{F}_{W}$

\subsection{Folheação infinitesimal}

Seja $V$ um espaço vetorial. Uma folheação $\mathcal{F}$ de $V$ será dita uma folheação infinitesimal se e somente se $\{0\}$ é uma folha e $\mathcal{F}$ é invariante pela multiplicação por escalar (invariante por homotetias).

Quando $V$ está munido com um produto interno $\langle\cdot, \cdot\rangle$ é imediato do Lema de homotetia (Lema 2.11) que uma FRS de $V$ é infinitesimal se e somente se $\{0\} \in \mathcal{F}$, de modo que a condição de invariância homotética é automaticamente satisfeita devido a estrutura métrica da folheação. Ao longo desta seção somente será abordado o caso no qual $V$ está munido com um produto interno e as folheações de $V$ são Riemannianas. Um exemplo interessante de folheação infinitesimal não Riemanniana é dada pela folheação do plano $\mathbb{R}^{2}$ por hiperbolóides, onde a "origem" e as "semi-retas coordenadas" são folhas.

Lema 2.16) Seja $\mathcal{F}$ uma FRS infinitesimal de $V$. Então $\mathcal{F}$ é uma subfolheação da folheação de $V$ dada por esferas centradas na origem.

Idéia da demonstração. Dado que $\mathcal{F}$ é Riemanniana segue, pela Proposição 2.5 , que $\mathcal{F}$ é localmente equidistante, de modo que ao menos uma folha de $\mathcal{F}$ está contida em uma esfera de $V$, uma vez que $\{0\} \in \mathcal{F}$.

Assim, pela invariância por homotetia, segue que todas as folhas de $\mathcal{F}$ estão contidas em esferas de $V$, ou seja, $\mathcal{F}$ é uma subfolheação da folheação de $V$ dada por esferas centradas na origem.

Dado que, para uma FRS infinitesimal $\mathcal{F}$, a folha $\{0\}$ é fechada, é possível linearizar campos e a própria folheação $\mathcal{F}$ em torno de $\{0\}$. Neste caso, o submódulo dos campos linearizados tangentes à folheação possui uma caracterização interessante e de suma importância para a continuidade do texto, a qual será apresentada nas Proposições 2.17 e 2.18.

Proposição 2.17) (Linearização de campos em FRS infinitesimais) Seja $\mathcal{F}$ uma FRS infinitesmial de $V$ e considere $\mathfrak{X}_{\text {Kil. }}(V) \subset \mathfrak{X}(V)$ a subálgebra de Lie dos campos de Killing de $V$. Então 
a) $\left.\quad X^{\ell}\right|_{v}=\left.\nabla_{v} X\right|_{0}$, para todo $v \in V$ e $X \in \mathfrak{X}(V,\{0\})=\left\{X \in \mathfrak{X}(V):\left.X\right|_{0}=0\right\}$. Em particular, $X^{\ell}$ é dada por uma aplicação linear.

b) $\mathfrak{X}_{\text {Kil. }}(V) \cap \mathfrak{X}(\mathcal{F})=\mathfrak{X}(\mathcal{F})^{\ell}$. Em particular, $X^{\ell}$ é um campo de Killing, para todo $X \in$ $\mathfrak{X}(\mathcal{F})$.

\section{Demonstração.}

a) Seja $X \in \mathfrak{X}(V,\{0\})$. Uma vez que $h_{\lambda}^{-1}$ é linear, tem-se que

$$
\left.X^{\ell}\right|_{v}=\left.\lim _{\lambda \rightarrow 0} \mathrm{~d} h_{\lambda}^{-1}\right|_{h_{\lambda}(v)}\left(\left.X\right|_{h_{\lambda}(v)}\right)=\lim _{\lambda \rightarrow 0} h_{\lambda}^{-1}\left(\left.X\right|_{h_{\lambda}(v)}\right)=\lim _{\lambda \rightarrow 0} \frac{\left.X\right|_{\lambda v}-\left.X\right|_{0}}{\lambda}=\left.\nabla_{v} X\right|_{0},
$$

para todo $v \in V$.

b) Seja $X \in \mathfrak{X}_{\text {Kil. }}(V) \cap \mathfrak{X}(\mathcal{F})$. Então, como $X$ é um campo de Killing, tal que $\left.X\right|_{0}=0$, tem-se que $X$ é um campo linear dado por uma matriz anti-simétrica, de modo que $\left.\nabla_{v} X\right|_{0}=\left.X\right|_{v}$.

Logo, pelo item anterior, tem-se que $\left.X^{\ell}\right|_{v}=\left.\nabla_{v} X\right|_{0}=\left.X\right|_{v}$, para todo $v \in V$, ou seja, $X=X^{\ell} \in \mathfrak{X}(\mathcal{F})^{\ell}$.

Assim $\mathfrak{X}_{\text {Kil. }}(V) \cap \mathfrak{X}(\mathcal{F}) \subset \mathfrak{X}(\mathcal{F})^{\ell}$.

Seja $X^{\ell} \in \mathfrak{X}(\mathcal{F})^{\ell}$ e considere $T \in \operatorname{End}(V)$ dada por $T(v):=\left.\nabla_{v} X\right|_{0}$.

Então $T$ é anti-simétrica. De fato, como $X^{\ell} \in \mathfrak{X}(\mathcal{F})$ e $\mathcal{F}$ é uma subfolheação da folheação de $V$ dada por esferas centradas em 0 , tem-se que $\left\langle X^{\ell}, v\right\rangle=0$, para todo $v \in V$ e, pelo item anterior, segue que $\langle T(v), v\rangle=\left\langle\left.\nabla_{v} X\right|_{0}, v\right\rangle=0$, para todo $v \in V$.

Logo, pelo item anterior, $\left.X^{\ell}\right|_{v}=T(v)$, para todo $v \in V$, com $T$ anti-simétrica, ou seja, $X^{\ell}$ é um campo de Killing.

$\operatorname{Assim} \mathfrak{X}_{\text {Kil. }}(V) \cap \mathfrak{X}(\mathcal{F}) \supset \mathfrak{X}(\mathcal{F})^{\ell}$.

Além da caracterização do submódulo $\mathfrak{X}(\mathcal{F})^{\ell}$ dos campos linearizados tangentes à folheação, a linearização de FRS infinitesimais possue propriedades importantes. Será mostrado, na Proposição 2.19 , que $\mathcal{F}^{\ell}$ é homogênea (relembre a definição de folheação homogênea nos exemplos da Seção 2.1.1, se necessário).

Proposição 2.18) Seja $\mathcal{F}$ uma FRS infinitesmial de $V$ e considere Iso $(V)$ o grupo de isometrias de $(V,\langle\cdot, \cdot\rangle)$,

$$
\operatorname{Iso}(\mathcal{F})=\{T \in \operatorname{Iso}(V): T(L) \subset L, \forall L \in \mathcal{F}\}
$$

e $\mathrm{K}^{0}$ a componente conexa de $\operatorname{Iso}(\mathcal{F})$ em torno da identidade. Então $\mathrm{K}^{0} \subset \mathrm{Iso}(V)$ é um subgrupo de Lie conexo, com álgebra de Lie dada por Lie $\left(\mathrm{K}^{0}\right)=\mathfrak{X}_{\mathrm{Kil}}(V) \cap \mathfrak{X}(\mathcal{F})$.

Ainda

$$
\mathrm{K}^{0}=\left\{\phi_{t}^{X^{\ell}} \in \operatorname{Iso}(V): n \in \mathbb{Z}_{+}^{*}, X \in \mathfrak{X}(\mathcal{F})^{n}, t \in \mathbb{R}^{n}\right\}
$$


onde $\phi_{t}^{X^{\ell}}=\phi_{t_{n}}^{X_{n}^{\ell}} \circ \cdots \circ \phi_{t_{1}}^{X_{1}^{\ell}}$ é a composta dos fluxos de $X^{\ell}=\left(X_{1}^{\ell}, \cdots, X_{n}^{\ell}\right)$ nos instantes $t=\left(t_{1}, \cdots, t_{n}\right)$.

Demonstração. Uma vez que $\mathfrak{X}_{\text {Kil. }}(V)$ e $\mathfrak{X}(\mathcal{F})$ são subálgebras de Lie de $\mathfrak{X}(V)$, segue que $\mathfrak{X}_{\text {Kil. }}(V) \cap \mathfrak{X}(\mathcal{F})$ é uma subálgebra de Lie de $\mathfrak{X}_{\text {Kil. }}(V)$ e, portanto, $\mathrm{K}^{0} \subset \mathrm{Iso}(V)$ é um único subgrupo de Lie conexo, tal que Lie $\left(\mathrm{K}^{0}\right)=\mathfrak{X}_{\text {Kil. }}(V) \cap \mathfrak{X}(\mathcal{F})$.

Ainda, como $\mathfrak{X}(\mathcal{F})^{\ell} \subset \mathfrak{X}_{\text {Kil. }}(V)$ é uma subálgebra de Lie, o subgrupo

$$
H=\left\{\phi_{t_{n}}^{n} \circ \cdots \circ \phi_{t_{1}}^{1} \in \operatorname{Iso}(V): n \in \mathbb{Z}_{+}^{*}, X_{i} \in \mathfrak{X}(\mathcal{F}), t_{i} \in \mathbb{R}, \forall i \in\{1, \cdots, n\}\right\}
$$

é o único subgrupo de Lie conexo, tal que $\operatorname{Lie}(H)=\mathfrak{X}(\mathcal{F})^{\ell}$.

Assim, como $\mathfrak{X}_{\text {Kil. }}(V) \cap \mathfrak{X}(\mathcal{F})=\mathfrak{X}(\mathcal{F})^{\ell}$, segue que

$$
\mathrm{K}^{0}=H=\left\{\phi_{t}^{X^{\ell}} \in \operatorname{Iso}(V): n \in \mathbb{Z}_{+}^{*}, X \in \mathfrak{X}(\mathcal{F})^{n}, t \in \mathbb{R}^{n}\right\}
$$

Observação) Relembre (ver Teorema 8.1.6 de [28], por exemplo) que, em uma variedade Riemanianna completa $(M, g)$, o grupo de isometrias Iso $(M)$ é um grupo de Lie, com álgebra de Lie dada pelo conjunto dos campos de Killing de $M$. Neste caso a aplicação exponencial é dada por $\exp (X):=\phi(1)$, para todo campo de Killing $X$ com fluxo $\phi$. Em particular, dado um espaço vetorial $V$ munido com um produto interno $\langle\cdot, \cdot\rangle$, o grupo Iso $(V)$ das isometrias (lineares) de $V$ é um grupo de Lie, com álgebra de Lie dado pelos campos de Killing de $V$.

Ainda, relembre (ver Teorema 1.10.3 de [13], por exemplo) que dados um grupo de lie G, com álgebra de Lie $\mathfrak{g}, \mathrm{e} \mathfrak{h} \subset \mathfrak{g}$ um subálgebra de Lie, existe um único subgrupo de Lie conexo $H \subset G$, tal que a álgebra de Lie de $H$ é hy, a saber, $H$ é gerado (como grupo) por exp $(H)$, onde exp é a aplicação exponencial de $G$.

Corolário 2.19) (Linearização de FRS infinitesimais) Seja $\mathcal{F}$ uma FRS infinitesmial de $V$ e considere $\mathcal{F}^{\ell}$ a linerização de $\mathcal{F}$ em torno de $\{0\}$. Então $\mathcal{F}^{\ell}$ é homogênea e dada por órbitas de $\mathrm{K}^{0}$.

Demonstração. Considere $\mathfrak{X}^{\mathrm{K}^{0}}(V):=\left\{X^{*} \in \mathfrak{X}(V): X \in\right.$ Lie $\left.\left(\mathrm{K}^{0}\right)\right\}$ o submódulo dos campos infinitesimais da ação isométrica de $\mathrm{K}^{0}$ sobre $V$ (ver Seção 1.1.1, se for necessário relembrar o conceito) e $\mathcal{F}^{\mathrm{K}^{0}}$ a folheação de $V$ dada pelas órbitas de $\mathrm{K}^{0}$.

Relembre que $\mathfrak{X}^{\mathrm{K}^{0}}(V)$ é isomorfo a álgebra de Lie de $\mathrm{K}^{0}$ e a folheação gerada pela aplicação do Teorema de Sussmann ao submódulo $\mathfrak{X}^{\mathrm{K}^{0}}(V)$ é exatamente a folheação $\mathcal{F}^{\mathrm{K}^{0}}$.

Assim, pela Proposição 2.17 e pela proposição anterior, segue que

$$
\mathfrak{X}^{\mathrm{K}^{0}}(V)=\mathfrak{X}_{\mathrm{Kil}}(V) \cap \mathfrak{X}(\mathcal{F})=\mathfrak{X}(\mathcal{F})^{\ell}
$$

e, portanto, $\mathcal{F}^{\mathrm{K}^{0}}=\mathcal{F}^{\ell}$.

Dada $(M, g)$ uma variedade Riemanniana e $\mathcal{F}$ uma FRS de $M$, para cada $p \in M$, é possível associar uma FRS infinitesimal de $\left(\nu_{p} L_{p},\left.g_{p}\right|_{\nu_{p} L_{p}}\right)$. 
Dado que uma seção tranversal normal (local) $S_{p}$ é sempre transversal as folhas de $\mathcal{F}$, segue que a partição $\mathcal{F}_{S_{p}}$ dada pelas componentes conexas da intersecção de $S_{p}$ com as folhas de $\mathcal{F}$ é uma folheação singular. Devido à invariância por homotetias, é possível extender por homotetias a folheação $\left(\exp _{p}\right)^{*} \mathcal{F}_{S_{p}}$ a uma folheação $\mathcal{F}_{p}$ de $\nu_{p} L_{p}$, de modo que $\left(\nu_{p} L_{p}, \mathcal{F}_{p},\left.g_{p}\right|_{\nu_{p} L_{p}}\right)$ é uma FRS infinitesimal que será dita a folheação infinitesimal de $\mathcal{F}$ em $p$.

Proposição 2.20) (Componente fundamental da folheação infinitesimal) Seja $B \subset M$ uma subvariedade fechada, saturada e contida em um estrato. Então

$$
\left(\nu_{b} L_{b}, \mathcal{F}_{b},\left.g_{b}\right|_{\nu_{b}} L_{b}\right) \approx\left(\nu_{b} L_{b} \cap \mathrm{T}_{b} B,\{\text { pts. }\}\right) \times\left(\nu_{b} B,\left.\mathcal{F}_{b}\right|_{\nu_{b} B}\right)
$$

para todo $b \in B$.

Uma prova da Proposição anterior pode ser encontrada em [7], por exemplo. Motivando-se por esta proposição, quando $B \subset M$ for uma subvariedade fechada, saturada e contida em um estrato, para cada $b \in B$, por um abuso de notação, a folheação $\left.\mathcal{F}_{b}\right|_{\nu_{b} B}$ será dita a folheação infinitesimal de $\mathcal{F}$ em $b$ e será denotada por $\mathcal{F}_{b}$.

A seguir será ilustrado, em um exemplo, a idéia por trás desta decomposição da folheação infinitesimal.

Exemplo) Seja $\mathcal{F}$ a folheação de $\mathbb{R}^{3}$ por círculos contidos nos planos paralelos ao "plano YZ" (denotado por $Y 0 Z$ ) e centrados em torno do "eixo X" (denotado por $0 X$ ), de modo que os pontos do eixo $X$ são as folhas singulares de $\mathcal{F}$. Considere $B$ o estrato mínimo de $\mathcal{F}$, ou seja, o próprio "eixo X".

Então $\mathcal{F}_{b}=\mathcal{F}$, para todo $b \in B$, uma vez que o próprio $\mathbb{R}^{3}$ é uma seção normal de $\mathcal{F}$ passando por $b$. Ainda, como $\nu_{b} B$ é o plano paralelo ao "plano YZ" passando por $b$, tem-se que $\left.\mathcal{F}_{b}\right|_{\nu_{b} B}$ é a folheação deste plano por círculos.

$\operatorname{Assim}\left(\mathbb{R}^{3}, \mathcal{F}_{b}\right) \approx(0 X,\{$ pts. $\}) \times\left(Y 0 Z,\left.\mathcal{F}_{b}\right|_{\nu_{b} B}\right)$.

Lema 2.21) Sejam $B \subset M$ uma subvariedade fechada, saturada e contida em um estrato e $W=\exp (\mathcal{W})$ uma VTN em torno de $B$. Então, para cada $b \in B$,

a) dado $X^{b} \in \mathfrak{X}\left(\nu_{b} B,\{0\}\right)$, existe $X \in \mathfrak{X}(W, B)$, tal que $\left.X^{\ell}\right|_{W_{b}}=\left.\left(X^{b}\right)^{\ell}\right|_{\mathcal{W}_{b}}$ e $\left.X^{\ell}\right|_{W_{\tilde{b}}} \in$ $\mathfrak{X}^{\ell}\left(W_{\tilde{b}}\right)$, para todo $\tilde{b} \in B$;

b) dado $X^{b} \in \mathfrak{X}\left(\mathcal{F}_{b}\right)$, existe $X \in \mathfrak{X}\left(\mathcal{F}_{W}\right)$, tal que $\left.X^{\ell}\right|_{W_{b}}=\left.\left(X^{b}\right)^{\ell}\right|_{\mathcal{W}_{b}}$ e $\left.X^{\ell}\right|_{W_{\tilde{b}}} \in \mathfrak{X}^{\ell}\left(\mathcal{F}_{\tilde{b}}\right)$, para todo $\tilde{b} \in B$;

c) dado $k_{b} \in \mathrm{K}_{b}^{0}$, existem $n \in \mathbb{Z}_{+}^{*}, X \in \mathfrak{X}\left(\mathcal{F}_{W}\right)^{n}$ e $t \in \mathbb{R}^{n}$, tal que $k_{b}=\left.\phi_{t}^{X^{\ell}}\right|_{W_{b}}$, onde $X^{\ell}$ é a extensão a $\nu B$ da linearização da $n$-upla de campos $X$.

\section{Demonstração.}


a) Considere $X \in \mathfrak{X}(W, B)$, tal que $\left.X\right|_{B} \equiv 0$ e $\left.X\right|_{W_{b}}=X^{b}$ ( $X$ pode ser construído em uma vizinhança trivializadora e extendido por uma "bump-function" de $B$ em torno de $b$ ).

Dado que $X^{\ell}$ é $\rho$-projetável, tem-se que $\left.\mathrm{d} \rho\right|_{p}\left(\left.X^{\ell}\right|_{p}\right)=\left.X\right|_{\rho(p)}=0$, ou seja, $\left.X^{\ell}\right|_{W_{\tilde{b}}} \in$ $\mathfrak{X}\left(W_{\tilde{b}}\right)$, para todo $\tilde{b} \in B$. Ainda, como $X^{\ell}$ é invariante por homotetias, $\left.X^{\ell}\right|_{W_{\tilde{b}}}$ é invariante por homotetias (em $W_{\tilde{b}}$ ), ou seja, $\left.X^{\ell}\right|_{W_{\tilde{b}}}$ é linear, para todo $\tilde{b} \in B$.

Por fim,

$$
\left.\left(X^{b}\right)^{\ell}\right|_{p}=\lim _{\lambda \rightarrow 0} \mathrm{~d} h_{\lambda}^{-1}\left(\left.X^{b}\right|_{h_{\lambda}(p)}\right)=\lim _{\lambda \rightarrow 0} \mathrm{~d} h_{\lambda}^{-1}\left(\left.X\right|_{h_{\lambda}(p)}\right)=\left.X^{\ell}\right|_{p}=\left.\left(\left.X^{\ell}\right|_{W_{b}}\right)\right|_{p}
$$

b) Considere $X \in \mathfrak{X}\left(\mathcal{F}_{W}\right)$, tal que $\left.X\right|_{B} \equiv 0$ e $\left.X\right|_{W_{b}}=X^{b}$ (X pode ser construído extendendo-se $X^{b}$ por meio do fluxo de um campo linearizado em uma vizinhança de $W$ e extendido por uma "bump-function" de $B$ em torno de $b$ ).

Procedendo-se de maneira análoga ao feito no item anterior, obtém-se que $\left.X^{\ell}\right|_{W_{b}}=$ $\left.\left(X^{b}\right)^{\ell}\right|_{\mathcal{W}_{b}}$ e $\left.X^{\ell}\right|_{W_{\tilde{b}}} \in \mathfrak{X}^{\ell}\left(\mathcal{F}_{\tilde{b}}\right)$, para todo $\tilde{b} \in B$.

c) Segue como consequência da Proposição 2.18 e do item anterior.

Uma caracterização importante da linearização de FRS, que evidência ainda mais o seu caráter intrínseco, será apresentada a seguir. Em linhas gerais, pode-se dizer que $\mathcal{F}^{\ell}$ é a "maior" subfolheação transversalmente homogênea de $\mathcal{F}$ em $W$.

Proposição 2.22) Sejam $B \subset M$ uma subvariedade fechada, saturada e contida em um estrato e $W=\exp (\mathcal{W})$ uma VTN em torno de $B$. Então, para cada $b \in B$,

a) $\left(\mathcal{F}^{\ell}\right)_{b}=\left(\mathcal{F}_{b}\right)^{\ell}$, onde $\left(\mathcal{F}_{b}\right)^{\ell}$ é a linearização de $\mathcal{F}_{b}$ com relação a $\{0\}$;

b) $\left(\mathcal{F}^{\ell}\right)_{b}$ é homogênea e dada por órbitas de $K_{b}^{0}$.

\section{Demonstração.}

a) Ver [23].

b) Uma vez que, para cada $b \in B, \mathcal{F}_{b}$ é uma FRS (em $\nu_{b} B$ ), tal que $\{0\} \in \mathcal{F}_{b}$, segue, da Proposição 2.19, que $\left(\mathcal{F}_{b}\right)^{\ell}$ é homogênea e dada por órbitas do grupo $\mathrm{K}_{b}^{0}$.

Assim, pelo item anterior, $\left(\mathcal{F}^{\ell}\right)_{b}$ é homogênea e dada por órbitas de $\mathrm{K}_{b}^{0}$.

\subsection{Folheações de tipo órbita e folheação de fecho local}

Dado $(M, g)$ uma variedade Riemanniana. Uma FRS $\mathcal{F}$ de $M$ será dita transversalmente $\underline{\text { (ou infinitesimalmente) homogênea se e somente se } \mathcal{F}_{p} \text { é uma FRS homogênea (i.e. dada }}$ 
por órbitas de uma ação isométrica), para todo $p \in M$, de modo que o item (b) da Proposição 2.22 no fim da última seção mostra que $\mathcal{F}^{\ell}$ é transversalmente homogênea.

Um tipo mais restrito de FRS apresentado por Molino, em [24], é a folheação de tipo órbita. Uma FRS $\mathcal{F}$ será dita de tipo órbita se e somente se $\mathcal{F}_{p}$ é uma FRS homogênea e fechada, para todo $p \in M$. Note que, neste caso, $\mathcal{F}^{\ell}$ não é necessariamente uma folheação de tipo órbita, dado que as folheações infinitesimais de $\mathcal{F}^{\ell}$ podem não ser fechadas.

Motivando-se por este fato, dado $W$ uma VTN em torno de uma subvariedade fechada, saturada e contida em um estrato, defina para cada $p \in W$,

$$
L_{p}^{\text {lc }}:=\left\{\phi_{t}^{X^{\ell}}(k \cdot p) \in M: n \in \mathbb{Z}_{+}^{*}, X \in \mathfrak{X}\left(\mathcal{F}_{W}\right)^{n}, t \in \mathbb{R}^{n}, k \in \bar{K}_{\pi(p)}^{0}\right\}
$$

Então $\mathcal{F}^{\text {lc }}:=\left\{L_{p}^{\text {lc }}\right\}_{p \in W}$ é uma folheação singular que será denominada a folheação de fecho local de $\mathcal{F}$ em $W$.

São válidos para as folheações infinitesimais de $\mathcal{F}^{\text {lc }}$ resultados análogos aos da folheação linearizada $\mathcal{F}^{\ell}$, por exemplo, a Proposição 2.22 , de modo que $\mathcal{F}^{\text {lc }}$ é uma FRS de tipo órbita, tal que $\mathcal{F}^{\ell}<\mathcal{F}^{\text {lc }}$ e $\overline{\mathcal{F}^{\ell}}=\overline{\mathcal{F}^{\text {lc }}}$.

O capítulo se encerra, então, apresentando um teorema a cerca das folheações de fecho local, que mostra a sua importância na descrição da dinâmica do fecho de $\mathcal{F}$ em uma VTN $W$.

Teorema 2.23) ([7]) Sejam $(M, g)$ uma variedade Riemanniana e $\mathcal{F}$ uma FRS de $M$. Então, dada $L \in \mathcal{F}$, existe $W$ uma $\varepsilon$-vizinhança tubular em torno de $\bar{L}$ e $g^{\ell}$ uma métrica em $W$, tal que
a) $\left(W, g^{\ell}, \mathcal{F}^{\text {lc }}\right)$ é uma FRS de tipo órbita.
b) $\left.\mathcal{F}^{\mathrm{lc}}\right|_{\bar{L}}=\left.\mathcal{F}\right|_{\bar{L}}$.
c) O fecho de $\mathcal{F}^{\text {lc }}$ está contido no fecho de $\mathcal{F}$. 


\section{Capítulo 3.}

\section{Grupóides de Lie}

O Capítulo 3 fará uma breve e rudimentar exposição do conceito de grupóides de Lie e de seus morfismos (Seção 3.1). Serão, ainda, apresentandos alguns exemplos (Seção 3.2) e construções (Seção 3.3) com intuito de estabelecer notação, linguagem e certa compreensão necessária para a leitura dos Capítulos 4 e 5. A exposição de cada um dos exemplos e de cada uma das construções, claramente, estará dividida em duas partes, a saber, a parte algébrica e a parte topológica e diferenciável.

O leitor pode ignorar a prova dos lemas presentes neste capítulo, se assim o quiser, sem se preocupar com prejuízos futuros à compreensão do texto.

\subsection{Grupóides de Lie e morfismo de grupóides de Lie}

Um grupóide é uma categoria pequena, onde todos os morfismo são invertíveis. Equivalentemente, de modo mais concreto e menos sucinto, um grupóide pode ser visto como um par de conjuntos $\left(\mathcal{G}_{0}, \mathcal{G}_{1}\right)$, munidos de aplicações

- ("source" e "target") $s, t: \mathcal{G}_{1} \longrightarrow \mathcal{G}_{0}$,

- (multiplicação) $\mathrm{m}: \mathcal{G}_{1}{ }_{s} \times{ }_{t} \mathcal{G}_{1} \longrightarrow \mathcal{G}_{1}, \operatorname{com} \mathcal{G}_{1 s} \times{ }_{t} \mathcal{G}_{1}:=\left\{(v, u) \in \mathcal{G}_{1} \times \mathcal{G}_{1}: s(v)=t(u)\right\}$,

- (seção unitária) $\mathbb{1}: \mathcal{G}_{0} \longrightarrow \mathcal{G}_{1}$,

- (inversão) $i: \mathcal{G}_{1} \longrightarrow \mathcal{G}_{1}$,

satisfazendo as seguintes propriedades

- (associatividade da multiplicação) $s(\mathrm{~m}(v, u))=s(u), t(\mathrm{~m}(v, u))=t(v) \mathrm{e}$

$$
\mathrm{m}(w, \mathrm{~m}(v, u))=\mathrm{m}(\mathrm{m}(w, v), u)
$$

para todo $u, v, w \in \mathcal{G}_{1}$, tais que $s(v)=t(u)$ e $s(w)=t(v)$;

- (elemento neutro da multiplicação) $s \circ \mathbb{1}=t \circ \mathbb{1}=\mathrm{Id}$ (em particular, $s$ e $t$ são sobrejetoras e $\mathbb{1}$ é injetora) e

$$
\mathrm{m}(u, \mathbb{1}(s(u)))=\mathrm{m}(\mathbb{1}(t(u)), u)=u,
$$

para todo $u \in \mathcal{G}_{1}$; 
- (elemento neutro da inversão) $s \circ i=t, t \circ i=s \mathrm{e}$

$$
\mathrm{m}(i(u), u)=\mathbb{1}(s(u)), \quad \mathrm{m}(u, i(u))=\mathbb{1}(t(u)),
$$

para todo $u \in \mathcal{G}_{1}$.

Dados $x \in \mathcal{G}_{0}$ e $u, v \in \mathcal{G}_{1}$, tais que $s(v)=t(u)$, comumente, por fins de simplicidade, $\mathbb{1}(x)$, $\mathrm{m}(v, u)$ e $i(u)$ serão denotados por $1_{x}, v \cdot u$ e $u^{-1}$, respectivamente.

Vale ressaltar que a partir das propriedades descritas na definição de um grupóide, é possível provar (ver [18], por exemplo) a unicidade dos elementos neutro e inverso da multiplicação, ou seja, dados $u, v \in \mathcal{G}_{1}$, tais que $t(u)=s(v)$,

- (unicidade do elemento neutro da multiplicação) se $v \cdot u=v$ (resp. $v \cdot u=u$ ), então $u=1_{s(v)}\left(\right.$ resp. $\left.v=1_{t(u)}\right)$;

- (unicidade do elemento inverso da multiplicação) se $v \cdot u=1_{t(v)}\left(\right.$ resp. $\left.v \cdot u=1_{s(u)}\right)$, então $u=v^{-1}$ (resp. $v=u^{-1}$ ). Ainda, em particular, $\left(u^{-1}\right)^{-1}=u$, para todo $u \in \mathcal{G}_{1}$.

O conjunto $\mathcal{G}_{0}$ será dito o conjunto de objetos do grupóide $\mathcal{G}$, o conjunto $\mathcal{G}_{1}$ será dito o conjunto de flechas do grupóide $\mathcal{G}$ e $s, t, \mathbb{1}, \mathrm{m}$ e $i$ serão ditas as aplicações estruturais ou operações do grupóide $\mathcal{G}$. Usualmente, um grupóide $\mathcal{G}$ será denotado por $\mathcal{G}_{1} \rightrightarrows \mathcal{G}_{0}$.

Dado $x \in \mathcal{G}_{0}$ o conjunto

$$
\mathcal{G}_{x}:=s^{-1}(\{x\}) \cap t^{-1}(\{x\})=\left\{u \in \mathcal{G}_{1}: s(u)=t(u)=x\right\}
$$

possui uma estrutura de grupo dada pela restrição de m à $\mathcal{G}_{x} \times \mathcal{G}_{x}$. O grupo $\mathcal{G}_{x}$ será dito o $\underline{\text { grupo de isotropia do grupóide } \mathcal{G}}$ em $x$. Ainda, o conjunto

$$
\mathcal{G}(x):=t\left(s^{-1}(\{x\})\right)=\left\{t(u) \in \mathcal{G}_{0}: s(u)=x\right\}
$$

será denominado a órbita do grupóide $\mathcal{G}$ passando por $x$.

Um grupóide $\mathcal{G}$ será dito um grupóide de Lie se, e somente, se $\mathcal{G}_{0}$ for uma variedade, $\mathcal{G}_{1}$ for uma variedade (não necessariamente Hausdorff e segundo contável), $s$ ou $t$ forem submersões e $\mathbb{1}, \mathrm{m}$ e $i$ forem suaves. Vale ressaltar que

- $i$ é um difeomorfismo, dado que $i \circ i=\mathrm{Id}$;

- a condição de $s$ (resp. $t$ ) ser uma submersão implica, imediatamente, que $t$ (resp. $s$ ) é também uma submersão, dado que $s \circ i=t$ (resp. $t \circ i=s$ ) e $i$ é um difeomorfismo;

- $\mathbb{1}$ é uma seção global das submersões $s$ e $t$ e, em particular, é uma imersão.

Ainda, é crucial ressaltar que a exigência de $s$ ou $t$ serem submersões implica que $\mathcal{G}_{1}{ }_{s}{ }_{t} \mathcal{G}_{1}$ é uma subvariedade de $\mathcal{G}_{1} \times \mathcal{G}_{1}$ (ver Corolário 2.19 em [16], por exemplo), de modo que a exigência da suavidade da multiplicação $m$ está bem posta. 
Quando $\mathcal{G}$ é um grupóide de Lie, os grupos de isotropia de $\mathcal{G}$ são grupos de Lie (ver [11], por exemplo) e as órbitas de $\mathcal{G}$ são subvariedades imersas (sem autointersecções) de $\mathcal{G}_{0}$, de modo que a partição de $\mathcal{G}_{0}$ dada pelas componentes conexas das órbitas de $\mathcal{G}$ formam uma folheação singular (ver [12], por exemplo).

Sejam $\mathcal{G}$ e $\widetilde{\mathcal{G}}$ grupóides. Um morfismo de grupóides entre $\mathcal{G}$ e $\widetilde{\mathcal{G}}$ é um par $\Phi=\left(\Phi_{1}, \Phi_{0}\right)$ de aplicações $\Phi_{0}: \mathcal{G}_{0} \longrightarrow \widetilde{\mathcal{G}_{0}}$ e $\Phi_{1}: \mathcal{G}_{1} \longrightarrow \widetilde{\mathcal{G}_{1}}$ invariantes pelas operações do grupóide, ou seja, tais que

- $\tilde{s} \circ \Phi_{1}=\Phi_{0} \circ s$ e $\tilde{t} \circ \Phi_{1}=\Phi_{0} \circ t$,

- $\Phi_{1} \circ \mathbb{1}=\tilde{\mathbb{1}} \circ \Phi_{0}$,

- $\Phi_{1} \circ \mathrm{m}=\left.\widetilde{\mathrm{m}} \circ\left(\Phi_{1} \times \Phi_{1}\right)\right|_{\mathcal{G}_{1 s} \times \mathcal{G}_{1}}$.

Observe que, como $\Phi$ é invariante pelo "source"e pelo "target" (primeira condição), tem-se que $\Phi_{1} \times \Phi_{1}\left(\mathcal{G}_{1} \times_{t} \mathcal{G}_{1}\right) \subset \widetilde{\mathcal{G}}_{\tilde{s}} \times \widetilde{\mathcal{G}}_{\tilde{t}}$, de modo que a terceira condição está bem colocada. Ainda, observe que as condições anteriores implicam que

- $\Phi_{1} \circ i=\tilde{i} \circ \Phi_{1}$.

Um morfismo de grupóides $\Phi$ (entre grupóides $\mathcal{G}$ e $\widetilde{\mathcal{G}}$ ), comumente, será denotado por $\Phi$ : $\mathcal{G} \longrightarrow \widetilde{\mathcal{G}}$.

A seguir será mostrado que a injetividade (resp. sobrejetividade) a nível de flechas implica a injetividade (resp. a sobrejetividade) a nível de objetos. Além disso, serão apresentadas condições para que a afirmação reversa seja verdadeira, ou seja, para que injetividade a nível de objetos implique a injetividade a nível de flechas.

Lema) Seja $\Phi: \mathcal{G} \longrightarrow \widetilde{\mathcal{G}}$ um morfismo de grupóides e considere

$$
\operatorname{Ker}(\Phi)=\left\{u \in \mathcal{G}_{1}: \Phi_{1}(u)=1_{\Phi_{0}(s(u))}\right\}
$$

Então

a) Se $\Phi_{1}$ é injetora (resp. sobrejetora), então $\Phi_{0}$ é injetora (resp. sobrejetora).

b) $\Phi_{0}$ é injetora e $\operatorname{Ker}(\Phi)=\operatorname{Im}(\mathbb{1})$ se, e somente se, $\Phi_{1}$ é injetora.

\section{Demonstração.}

a) Suponha que $\Phi_{1}$ seja injetora.

Dados $x, y \in \mathcal{G}_{0}$, tal que $\Phi_{0}(x)=\Phi_{0}(y)$, segue que $\Phi_{1}\left(1_{x}\right)=1_{\Phi_{0}(x)}=1_{\Phi_{0}(y)}=\Phi_{1}\left(1_{y}\right)$. Logo, pela injetividade de $\Phi_{1}$ e de $\mathbb{1}$, tem-se que $x=y$.

Assim $\Phi_{0}$ é injetora.

Suponha que $\Phi_{1}$ é sobrejetora.

Dado $\tilde{x} \in \widetilde{\mathcal{G}}_{0}$, segue que existe $u \in \mathcal{G}_{1}$, tal que $\Phi_{1}(u)=1_{\tilde{x}}$. Logo $\Phi_{0}(s(u))=\tilde{s}\left(\Phi_{1}(u)\right)=\tilde{x}$. 
Assim $\Phi_{0}$ é sobrejetora.

b) Suponha que $\Phi_{0}$ é injetora e $\operatorname{Ker}(\Phi)=\operatorname{Im}(\mathbb{1})$.

Dados $u, v \in \mathcal{G}_{1}$, tais que $\Phi_{1}(u)=\Phi_{1}(v)$, segue que $\Phi_{0}(t(u))=\tilde{t}\left(\Phi_{1}(u)\right)=\tilde{t}\left(\Phi_{1}(v)\right)=$ $\Phi_{0}(t(v))$ e, como $\Phi_{0}$ é injetora, tem-se que $t(u)=t(v)$. Logo

$$
\Phi_{1}\left(v^{-1} \cdot u\right)=\Phi_{1}(v)^{-1} \cdot \Phi_{1}(u)=1_{\tilde{s}\left(\Phi_{1}(u)\right)}=1_{\Phi_{0}(s(u))}=1_{\Phi_{0}\left(s\left(v^{-1} \cdot u\right)\right)},
$$

ou seja, $v^{-1} \cdot u \in \operatorname{Ker}(\Phi)$ e, portanto, $v^{-1} \cdot u=1_{s(u)}$ e, pela unicidade do elemento inverso, conclui-se que $u=v$.

Assim $\Phi_{1}$ é injetora.

Suponha que $\Phi_{1}$ seja injetora.

Então, pelo intem anterior, $\Phi_{0}$ é injetora. É imediato que $\operatorname{Ker}(\Phi) \subset \operatorname{Im}(\mathbb{1})$, devido ao fato de que $\Phi_{1} \circ \mathbb{1}=\tilde{\mathbb{1}} \circ \Phi_{0}$. Ainda $\operatorname{Ker}(\Phi) \supset \operatorname{Im}(\mathbb{1})$, pois dado $u \in \operatorname{Ker}(\Phi)$, segue que $\Phi_{1}(u)=1_{\Phi_{0}(s(u))}=\Phi_{1}\left(1_{s(u)}\right)$ o que implica, pela injetividade de $\Phi_{1}$, que $u=1_{s(u)}$.

$\operatorname{Assim} \operatorname{Ker}(\Phi)=\operatorname{Im}(\mathbb{1})$.

Um morfismo de grupóides $\Phi$, em vista do item (a) da proposição anterior, será dito injetor (resp. sobrejetor) se, e somente se, $\Phi_{1}$ for injetora (resp. sobrejetora). Naturalmente, $\Phi$ será dita bijetora se for injetora e sobrejetora e, neste caso, o par $\Phi_{1}^{-1}: \widetilde{\mathcal{G}}_{1} \longrightarrow \mathcal{G}_{1}$ e $\Phi_{0}^{-1}: \widetilde{\mathcal{G}}_{0} \longrightarrow \mathcal{G}_{0}$ é um morfismo de grupóides que será denotado por $\Phi^{-1}$ e dito o inverso de $\Phi$. Ainda, neste caso, $\Phi$ será dito um isomorfismo de grupóides entre $\mathcal{G}$ e $\widetilde{\mathcal{G}}$.

$\operatorname{Dados} \mathcal{G}$ e $\widetilde{\mathcal{G}}$, um morfismo (resp. isomorfismo) de grupóides $\Phi: \mathcal{G} \longrightarrow \widetilde{\mathcal{G}}$ será dito um morfismo (resp. isomorfismo) de grupóides de Lie se, e somente se, $\Phi_{0}$ e $\Phi_{1}$ forem suaves (resp. difeomorfismos).

\subsection{Exemplos de grupóides de Lie}

\section{Exemplos triviais}

A seguir serão apresentados alguns exemplo simples de grupóides de Lie. Estes exemplos não terão relevância direta ao longo do texto, mas irão estabelecer uma notação útil para o decorrer do texto e poderão familiarizar o leitor com o conceito de grupóides de Lie.

a) (Variedades) Seja $M$ uma variedade diferenciável. Defina

- ("source" e "target") $s, t: M \longrightarrow M$, por $s=t:=\mathrm{Id}$,

- (multiplicação) $\mathrm{m}: M_{s} \times_{t} M \longrightarrow M$, por $\mathrm{m}(x, x):=x$,

- (seção identidade) $\mathbb{1}: M \longrightarrow M$, por $\mathbb{1}:=\mathrm{Id}$, 
- (inversão) $i: M \longrightarrow M$, por $i:=\mathrm{Id}$.

Então $M \rightrightarrows M$, com as aplicações estruturais definidas, é um grupóide de Lie.

b) (Grupo de Lie) Seja $G$ um grupo de Lie. Considere $e$ o elemento neutro de $G$.

- ("source" e "target") $s, t: G \longrightarrow\{e\}$, por $s=t:=e$,

- (multiplicação) $\mathrm{m}: G_{s} \times_{t} G \longrightarrow G$, por $\mathrm{m}(x, y):=x y$,

- (seção identidade) $\mathbb{1}:\{e\} \longrightarrow G$, por $\mathbb{1}:=e$,

- (inversão) $i: G \longrightarrow G$, por $i(x):=x^{-1}$.

Então $G \rightrightarrows\{e\}$, com as aplicações estruturais definidas, é um grupóide de Lie.

c) (Grupóide do par) Seja $M$ uma variedade diferenciável. Considere $\operatorname{pr}_{1}, \operatorname{pr}_{2}: M \times$ $M \longrightarrow M$ as projeções canônicas na primeira e na segunda componente do produto $M \times M$, respectivamente. Defina

- ("source" e "target") $s, t: M \times M \longrightarrow M$, por $s:=\operatorname{pr}_{1}$ e $t:=\operatorname{pr}_{2}$,

- (multiplicação) $\mathrm{m}:(M \times M)_{s} \times_{t}(M \times M) \longrightarrow M \times M$, por $\mathrm{m}\left(\left(x_{1}, x_{2}\right),\left(y_{1}, y_{2}\right)\right):=\left(x_{1}, y_{2}\right)$,

- (seção identidade) $\mathbb{1}: M \longrightarrow M \times M$, por $\mathbb{1}(x):=(x, x)$,

- (inversão) $i: M \times M \longrightarrow M \times M$, por $i\left(x_{1}, x_{2}\right):=\left(x_{2}, x_{1}\right)$.

Então $(M \times M) \rightrightarrows M$, com as aplicações estruturais definidas, é um grupóide de Lie denominado o grupóide do par de M.

d) (Fibrados vetoriais) Sejam $M$ uma variedade diferenciável e $E$ um fibrado vetorial sobre $M$, com projeção $\pi$. Defina

- ("source" e "target") $s, t: E \longrightarrow M$, por $s=t:=\pi$,

- (multiplicação) $\mathrm{m}: E_{s} \times_{t} E \longrightarrow M$, por $\mathrm{m}(x, y):=x+y$,

- (seção identidade) $\mathbb{1}: M \longrightarrow E$, por $\mathbb{1}(p):=0_{p}$,

- (inversão) $i: E \longrightarrow E$, por $i(x):=-x$.

Então $E \rightrightarrows M$, com as aplicações estruturais definidas, é um grupóide de Lie.

e) (Grupóide de ação) Sejam $M$ uma variedade diferenciável e $G$ um grupo de Lie agindo à esquerda (resp. à direita) sobre $M$, por meio de uma ação $\mu$. Defina

- ("source" e "target") $s, t: G \times M \longrightarrow M$, por $s:=\operatorname{pr}_{M}$ e $t:=\mu$, onde $\operatorname{pr}_{M}$ é a projeção canônica na componente $M$,

- (multiplicação) $\mathrm{m}:(G \times M)_{s} \times_{t}(G \times M) \longrightarrow G \times M$, por $\mathrm{m}((g, x),(h, y)):=$ $(g h, y)$,

- (seção identidade) $\mathbb{1}: M \longrightarrow G \times M$, por $\mathbb{1}(x):=(e, x)$, 
- (inversão) $i: G \times M \longrightarrow G \times M$, por $i(g, x):=\left(g^{-1}, \mu(g, x)\right)$.

Então $G \ltimes M:=G \times M \rightrightarrows M$ (resp. $M \rtimes G:=M \times G \rightrightarrows M$ ), com as aplicações estruturais definidas, é um grupóide de Lie denominado o grupóide de ação da ação à esquerda (resp. à direita) $\mu$.

\section{Grupóide linear geral}

Sejam $M$ uma variedade diferenciável e $E$ um fibrado vetorial sobre $M$, com projeção $\pi$.

Dados $p, q \in M$, considere $\mathbb{G} \mathbb{L}\left(E_{p}, E_{q}\right)$ o conjunto dos isomorfismos entre $E_{p}$ e $E_{q}$. Os elementos de $\mathbb{G L}\left(E_{p}, E_{q}\right)$ serão, genericamente, denotados por $T_{p q}$ a fim de evidenciar o domínio e o contradomínio da aplicação $T_{p q}$.

Defina

- ("source" e "target") $s, t: \cup_{p, q \in M} \mathbb{G} \mathbb{L}\left(E_{p}, E_{q}\right) \longrightarrow M$ por $s\left(T_{p q}\right):=p$ e $t\left(T_{p q}\right):=q$,

- (seção identidade) $\mathbb{1}: M \longrightarrow \cup_{p, q \in M} \mathbb{G} \mathbb{L}\left(E_{p}, E_{q}\right)$ por $\mathbb{1}(p):=\operatorname{Id}_{E_{p}}$,

- (multiplicação) m : $\cup_{p, q \in M} \mathbb{G L}\left(E_{p}, E_{q}\right)_{s} \times_{t} \cup_{p, q \in M} \mathbb{G L}\left(E_{p}, E_{q}\right) \longrightarrow \cup_{p, q \in M} \mathbb{G L}\left(E_{p}, E_{q}\right)$ por $\mathrm{m}\left(S_{\tilde{p} \tilde{q}}, T_{p q}\right):=S_{\tilde{p} \tilde{q}} \circ T_{p q}$

- (inversão) $i: \cup_{p, q \in M} \mathbb{G L}\left(E_{p}, E_{q}\right) \longrightarrow \cup_{p, q \in M} \mathbb{G} \mathbb{L}\left(E_{p}, E_{q}\right)$ por $i\left(T_{p q}\right):=T_{p q}^{-1}$.

Então $\mathbb{G L}(E):=\cup_{p, q \in M} \mathbb{G L}\left(E_{p}, E_{q}\right) \rightrightarrows M$, com as aplicações estruturais definidas, é um grupóide de Lie denominado o grupóide linear geral do fibrado vetorial $E$.

Observe que, para cada $p \in M$,

- o grupo de isotropia de $\mathbb{G L}(E)$ em $p$ é o grupo linear geral de $E_{p}$, ou seja, $\mathbb{G L}(E)_{p}=$ $\mathbb{G} \mathbb{L}\left(E_{p}\right) ;$

- a órbita de $\mathbb{G} L(E)$ passando por $p$ é toda a variedade $M$.

Dado um produto interno nas fibras de $E$, analogamente, define-se o grupóide $\mathbb{O}(E)$.

\section{Grupóide de holonomia de conexões parciais}

Sejam $M$ uma variedade diferenciável, $\mathrm{D} \subset \mathrm{T} M$ um subfibrado e $\mathrm{H}$ uma distribuição horizontal ao longo de $D$, ou seja, um subfibrado $\mathrm{H} \subset \mathrm{T} E$, tal que

- $\mathrm{H} \cap \operatorname{Ker}(\mathrm{d} \pi)=\{0\}$ e $\mathrm{d} \pi(\mathrm{H})=\mathrm{D}$ (equivalentemente $\left.\mathrm{d} \pi\right|_{\mathrm{H}}: \mathrm{H} \longrightarrow D$ é um isomorfismo);

- $\mathrm{H}$ é $\mathrm{m}_{\lambda}$-invariante, para todo $\lambda \in \mathbb{R}^{*}$, onde $\mathrm{m}_{\lambda}: E \longrightarrow E$ é o isomorfismo dado pela multiplicação pelo escalar $\lambda$.

Considerando-se $C_{\text {par. }}^{\infty}([0,1], D)$ o conjunto das curvas suaves por partes definidas no intervalo $[0,1]$ e tangentes a $D$, para cada $\alpha \in C_{\text {par. }}^{\infty}([0,1], D)$ e $x \in E$, existe uma única curva $\tilde{\alpha}_{x}:[0,1] \subset$ 
$\mathbb{R} \longrightarrow E$ tangente a $\mathrm{H}$, tal que $\tilde{\alpha}_{x}(0)=x$ e $\pi \circ \tilde{\alpha}_{x}=\alpha$, a saber, ela é dada como solução da seguinte EDO

$$
\left\{\begin{array}{l}
\left(\tilde{\alpha}, \tilde{\alpha}^{\prime}\right)=\left(\left.\mathrm{d} \pi\right|_{\mathrm{H}}\right)^{-1}\left(\alpha, \alpha^{\prime}\right), \\
\tilde{\alpha}(0)=x
\end{array}\right.
$$

O operador $\mathrm{P}_{\alpha}: E_{\alpha(0)} \longrightarrow E_{\alpha(1)}$ dado por $\mathrm{P}_{\alpha}(x):=\tilde{\alpha}_{x}(1)$ é um isomorfismo que será dito o operador de transporte paralelo ao longo de $\alpha$.

Cabe ressaltar que $\mathrm{H}$ associa-se biunivocamente a uma conexão parcial $\nabla^{\mathrm{H}}$, ou seja, uma aplicação $\nabla^{\mathrm{H}}: \Gamma(E) \times \mathfrak{X}(D) \longrightarrow \Gamma(E)$, tal que

- (linearidade) $\nabla_{X}^{\mathrm{H}}(\lambda S+T)=\lambda \nabla_{X}^{\mathrm{H}} S+\nabla_{X}^{\mathrm{H}} T$,

- $\left(\right.$ regra de Leibniz) $\nabla_{X}^{\mathrm{H}}(f S)=X(f) S+f \nabla_{X}^{\mathrm{H}} S$,

para todo $\lambda \in \mathbb{R}, S, T \in \Gamma(E), X \in \mathfrak{X}(D)$ e $f \in C^{\infty}(M)$.

Considere $\mathcal{P}^{\mathrm{H}}:=\left\{\mathrm{P}_{\alpha} \in \mathbb{G} \mathbb{L}(E)_{1}: \alpha \in C_{\text {par. }}^{\infty}([0,1], D)\right\}$ e defina

- ("source" e "target") $s, t: \mathcal{P}^{\mathrm{H}} \longrightarrow M$ por $s\left(\mathrm{P}_{\alpha}\right):=\alpha(0)$ e $t\left(\mathrm{P}_{\alpha}\right):=\alpha(1)$,

- (seção identidade) $\mathbb{1}: M \longrightarrow \mathcal{P}^{\mathrm{H}}$ por $\mathbb{1}(p):=\operatorname{Id}_{E_{p}}$,

- (multiplicação) $\mathrm{m}: \mathcal{P}^{\mathrm{H}}{ }_{s} \times{ }_{t} \mathcal{P}^{\mathrm{H}} \longrightarrow \mathcal{P}^{\mathrm{H}}$ por $\mathrm{m}\left(\mathrm{P}_{\beta}, \mathrm{P}_{\alpha}\right):=\mathrm{P}_{\beta * \alpha}$,

- (inversão) $i: \mathcal{P}^{\mathrm{H}} \longrightarrow \mathcal{P}^{\mathrm{H}}$ por $i\left(\mathrm{P}_{\alpha}\right):=\mathrm{P}_{\alpha^{-1}}$.

Então $\mathrm{Hol}^{\mathrm{H}}:=\mathcal{P}^{\mathrm{H}} \rightrightarrows M$, com as aplicações estruturais definidas, é um grupóide denominado o grupóide de holonomia da distribuição horizontal $\mathrm{H}$.

Quando $D$ é integrável, ou seja, quando $\mathrm{D}=\mathrm{T} \mathcal{F}_{M}$, com $\mathcal{F}_{M}$ uma folheação (regular) de $M, C_{\text {par. }}^{\infty}([0,1], D)$ será o conjunto das curvas suaves por partes em $M$ inteiramente contidas em folhas de $\mathcal{F}_{M}$ e será denotado por $C_{\text {par. }}^{\infty}\left([0,1], \mathcal{F}_{M}\right)$, de modo que

$$
C_{\text {par. }}^{\infty}\left([0,1], \mathcal{F}_{M}\right):=\left\{\alpha \in C_{\text {par. }}^{\infty}([0,1], M): \operatorname{Im}(\alpha) \subset L_{\alpha(0)}\right\}
$$

Neste caso, vale ressaltar que, para cada $L \in \mathcal{F}_{M},\left.\mathrm{H}\right|_{L}$ é uma distribuição horizontal em $\left.E\right|_{L}$ e, para cada $p \in M$,

- o grupo de isotropia de $\mathrm{Hol}^{\mathrm{H}}$ em $p$ é o grupo de holonomia em $p$, com relação a distribuição horizontal $\left.\mathrm{H}\right|_{L_{p}}$, ou seja, $\left(\mathrm{Hol}^{\mathrm{H}}\right)_{p}=\left.\operatorname{Hol}_{p}^{\mathrm{H}}\right|_{L_{p}}$,

- a órbita de $\mathrm{Hol}^{\mathrm{H}}$ passando por $p$ é a folha de $\mathcal{F}$ passando por $p$, ou seja, $\mathrm{Hol}^{\mathrm{H}}(p)=L_{p}$.

\section{Monodromia de folheações regulares}

Sejam $M$ uma variedade diferenciável e $\mathcal{F}$ uma folheação singular de $M$.

Dados $\alpha_{0}, \alpha_{1} \in C_{\text {par. }}^{\infty}([0,1], \mathcal{F})$, será dito que $\alpha_{0}$ é $\mathcal{F}$-homotópica a $\alpha_{1}$ se, e somente se, existe uma homotopia $H:[0,1] \times[0,1] \longrightarrow M$ entre $\alpha_{0}$ e $\alpha_{1}$, tal que $\alpha_{s} \in C_{\text {par. }}^{\infty}([0,1], \mathcal{F})$, onde $\alpha_{s}:=H(\cdot, s)$, para todo $s \in[0,1]$. 
Observe que esta condição é equivalente a exigir que a homotopia $H$ seja folheada, considerando-se $[0,1] \times[0,1]$ folheado pela folheação produto, com o primeiro termo folheado pelo espaço todo e o segundo termo folheado por pontos.

Quando $\alpha_{s}(0)$ e $\alpha_{s}(1)$ forem constantes, será dito que $\alpha_{0}$ e $\alpha_{1}$ são $\mathcal{F}$-homotópicas com pontos iniciais e finais fixos. Neste caso $H$ será uma homotopia em $L_{\alpha(0)}$.

A $\mathcal{F}$-homotopia (resp. $\mathcal{F}$-homotopia com pontos iniciais e finais fixos) definem uma relação de equivalência em $M$, ou seja, $\alpha$ será equivalente a $\beta$ se, e somente se, $\alpha$ for $\mathcal{F}$-homotópica a $\beta$ (resp. $\alpha$ for $\mathcal{F}$-homotópica a $\beta$ com pontos iniciais e finais fixos). Dado $\alpha \in C_{\text {par. }}^{\infty}([0,1], \mathcal{F})$ a classe de equivalência de $\alpha$, com respeito a ambas as relações, será denotada por $[\alpha]$. Ainda, o espaço quociente, com respeito a relação dada por $\mathcal{F}$-homotopias que fixam os pontos iniciais e finais das curvas, será denotado por $\Pi_{1}(\mathcal{F})$.

A partir deste ponto do texto, sempre que duas curvas forem ditas $\mathcal{F}$-homotópicas, será considerada uma $\mathcal{F}$-homotopia que fixa os pontos inciais e finais das curvas.

Defina

- ("source" e "target") $s: \Pi_{1}(\mathcal{F}) \longrightarrow M$ por $s([\alpha]):=\alpha(0)$ e $t([\alpha]):=\alpha(1)$,

- (seção identidade) $\mathbb{1}: M \longrightarrow \Pi_{1}(\mathcal{F})$ por $\mathbb{1}(p):=\left[c_{p}\right]$, onde $c_{p}$ é a curva constante em $p$,

- (multiplicação) $\mathrm{m}: \Pi_{1}(\mathcal{F})_{s} \times_{t} \Pi_{1}(\mathcal{F}) \longrightarrow \Pi_{1}(\mathcal{F})$ por $\mathrm{m}([\beta],[\alpha]):=[\beta * \alpha]$,

- (inversão) $i: \Pi_{1}(\mathcal{F}) \longrightarrow \Pi_{1}(\mathcal{F})$ por $i([\alpha]):=\left[\alpha^{-1}\right]$, onde $\alpha^{-1}$ é a curva reversa de $\alpha$, a saber, dada por $\alpha^{-1}(t):=\alpha(1-t)$.

Então $\operatorname{Mon}(\mathcal{F}):=\Pi_{1}(\mathcal{F}) \rightrightarrows M$, com as aplicações estruturais definidas, é um grupóide denominado o grupóide de monodromia da folheação $\mathcal{F}$.

Observe que, para cada $p \in M$,

- o grupo de isotropia de $\operatorname{Mon}(\mathcal{F})$ em $p$ é o grupo fundamental da folha $L_{p}$, ou seja, $\operatorname{Mon}(\mathcal{F})_{p}=\pi_{1}\left(L_{p}, p\right)$;

- a órbita de $\operatorname{Mon}(\mathcal{F})$ passando por $p$ é a folha de $\mathcal{F}$ passando por $p$, ou seja, $\operatorname{Mon}(\mathcal{F})(p)=$ $L_{p}$

Quando $\mathcal{F}$ é regular $\operatorname{Mon}(\mathcal{F})$ é um grupóide de Lie. A prova deste fato passa pela construção de cartas para o conjunto $\Pi_{1}(\mathcal{F})$, a qual será brevemente apresentada a seguir e pode ser encontrada com maiores detalhes em [22] e [15], por exemplo.

Dada $[\alpha] \in \Pi_{1}(\mathcal{F})$, considere $\varphi_{0}: U_{0} \subset M \longrightarrow \mathbb{R}^{n} \times \mathbb{R}^{k}$ e $\varphi_{1}: U_{1} \subset M \longrightarrow \mathbb{R}^{n} \times \mathbb{R}^{k}$ cartas folheadas em torno de $\alpha(0)=\varphi_{0}^{-1}(0,0)$ e $\alpha(1)=\varphi_{1}^{-1}(0,0)$, respectivamente, tais que

- $\varphi_{0}\left(U_{0}\right)=\widetilde{U}_{0} \times \widetilde{V}_{0}$ e $\varphi_{1}\left(U_{1}\right)=\widetilde{U}_{1} \times \widetilde{V}_{1}$

- $\widetilde{U}_{0}$ e $\widetilde{U}_{1}$ são convexos e $\widetilde{V}_{0}$ e $\widetilde{V}_{1}$ são conexos;

- a aplicação de holonomia $\varphi_{\alpha}: S_{\alpha(0)} \longrightarrow S_{\alpha(1)}$ é um difeomorfismo, onde $S_{\alpha(0)}=$ $\varphi_{0}^{-1}\left(\{0\} \times \widetilde{V}_{0}\right)$ e $S_{\alpha(1)}=\varphi_{1}^{-1}\left(\{0\} \times \widetilde{V}_{1}\right)$ são slices de $\mathcal{F}$ passando por $\alpha(0)$ e $\alpha(1)$, respectivamente. 
Ainda,

- dado $\left(\tilde{x}_{i}, \tilde{y}_{i}\right) \in \widetilde{U}_{i} \times \widetilde{V}_{i}$, considere $r_{\left(\tilde{x}_{i}, \tilde{y}_{i}\right)} \in C^{\infty}([0,1], \mathcal{F})$ a "reta" dada $r_{\left(\tilde{x}_{i}, \tilde{y}_{i}\right)}(t):=$ $\varphi_{i}^{-1}\left(t \tilde{x}_{i}, \tilde{y}_{i}\right)$, para cada $i \in\{0,1\}$;

- dado $\tilde{y}_{0} \in \widetilde{V}_{0}$, considere $\alpha_{\tilde{y}_{0}} \in C^{\infty}([0,1], \mathcal{F})$ a curva dada por $\alpha_{\tilde{y}_{0}}(t):=\varphi_{\alpha}^{t}\left(\varphi_{0}^{-1}\left(0, \tilde{y}_{0}\right)\right)$.

Então $\Phi: \widetilde{U}_{0} \times \widetilde{V}_{0} \times \widetilde{U}_{1} \subset \mathbb{R}^{n} \times \mathbb{R}^{k} \times \mathbb{R}^{n} \longrightarrow \Pi_{1}(\mathcal{F})$ dada por

$$
\Phi\left(\tilde{x}_{0}, \tilde{x}_{1}, \tilde{y}_{0}\right)=\left[r_{\left(\tilde{x}_{1}, \tilde{\varphi}_{\alpha}\left(\tilde{y}_{0}\right)\right)} * \alpha_{\tilde{y}_{0}} *\left(r_{\left(\tilde{x}_{0}, \tilde{y}_{0}\right)}\right)^{-1}\right]
$$

é uma parametrização de $\Pi_{1}(\mathcal{F})$, em torno de $[\alpha]=\Phi(0,0,0)$, cuja inversa é dada por

$$
\Phi^{-1}([\beta]):=\left(\varphi_{0}(\beta(0)), \operatorname{pr}_{n}\left(\varphi_{1}(\beta(1))\right)\right),
$$

onde $\operatorname{pr}_{n}: \mathbb{R}^{n} \times \mathbb{R}^{k} \longrightarrow \mathbb{R}^{n}$ é a projeção canônica.

\subsection{Construção de grupóides}

A presente seção apresentará duas construções de grupóides de Lie (pertinentes à compreensão deste texto), a saber, o grupóide de ação construído a partir de ações de grupóides de Lie sobre variedades, cujo paralelo com o caso de grupos de Lie se faz por meio de ações suaves, e o grupóide quociente construído a partir de ações livres e próprias de grupos de Lie sobre grupóides de Lie.

\subsubsection{Ação de grupóides e grupóide de ação}

Seja $\mathcal{G}_{1} \rightrightarrows \mathcal{G}_{0}$ um grupóide, $M$ um conjunto e $f: M \longrightarrow \mathcal{G}_{0}$ uma função. Uma ação de $\mathcal{G}$ sobre $M$ ao longo de $f$ (que será dito o mapa momento da ação $\mu$ ) é uma aplicação $\mu: \mathcal{G}_{1_{s}} \times{ }_{f} M \longrightarrow M$, tal que

- $f(\mu(u, p))=t(u)$, para todo $(u, p) \in \mathcal{G}_{1_{s}} \times_{f} M$,

- $\mu\left(1_{f(p)}, p\right)=p$, para todo $p \in M$,

- $\mu(v, \mu(u, p))=\mu(v \cdot u, p)$, para todo $(u, p) \in \mathcal{G}_{1_{s} \times}{ }_{f} M$ e $v \in \mathcal{G}_{1}$, tais que $s(v)=t(u)$.

Dada uma ação $\mu$ de $\mathcal{G}$ sobre $M$, para cada $p \in M$, será considerada $\mu^{p}: s^{-1}(\{f(p)\}) \longrightarrow M$ a aplicação dada por $\mu^{p}(u):=\mu(u, p)$ e, para cada $u \in \mathcal{G}_{1}$, será consdireada $\mu_{u}: f^{-1}(\{s(u)\}) \longrightarrow$ $f^{-1}(\{t(u)\})$ a aplicação dada por $\mu_{u}(p):=\mu(u, p)$.

Dada uma ação $\mu: \mathcal{G}_{1_{s}} \times{ }_{f} M \longrightarrow M$, defina

- ("source" e "target") $s^{\ltimes}, t^{\ltimes}: \mathcal{G}_{1_{s}} \times_{f} M \longrightarrow M$, por

$$
s^{\ltimes}:=\operatorname{pr}_{M}, \quad t^{\ltimes}:=\mu,
$$


onde $\operatorname{pr}_{M}: \mathcal{G}_{1_{s}} \times{ }_{f} M \longrightarrow M$ é a projeção canônica;

- (multiplicação) $\mathrm{m}^{\ltimes}:\left(\mathcal{G}_{1} \times_{f} M\right)_{s^{\ltimes}} \times_{t^{\ltimes}}\left(\mathcal{G}_{1_{s}} \times{ }_{f} M\right) \longrightarrow \mathcal{G}_{1_{s}} \times_{f} M$, por

$$
\mathrm{m}^{\ltimes}((v, q),(u, p)):=(v \cdot u, p) ;
$$

- (seção identidade) $\mathbb{1}^{\ltimes}: M \longrightarrow \mathcal{G}_{1_{s}} \times_{f} M$, por

$$
\mathbb{1}^{\ltimes}(p):=\left(1_{f(p)}, p\right) ;
$$

- (inversão) $i^{\ltimes}: \mathcal{G}_{1} \times_{f} M \longrightarrow \mathcal{G}_{1_{s}} \times_{f} M$, por

$$
i^{\ltimes}(u, p):=\left(u^{-1}, \mu(u, p)\right) .
$$

Seguindo a simplificação de notação adotada no início deste capítulo os elementos $\mathrm{m}^{\ltimes}((v, q),(u, p)), \mathbb{1}^{\ltimes}(p)$ e $i^{\ltimes}(u, p)$ serão, simplesmente, denotados por $(v, q) \cdot(u, p), 1_{p}$ e $(u, p)^{-1}$.

Lema) $\mathcal{G} \ltimes M:=\mathcal{G}_{1_{s}} \times_{f} M \rightrightarrows M$, com as operações descritas, é um grupóide.

Demonstração. Observe, primeiramente, que

- $\mathrm{m}^{\ltimes}$ está bem definida, pois dados $(u, p),(v, q) \in \mathcal{G}_{1_{s} \times}{ }_{f} M$, tais que $q=s^{\ltimes}(v, q)=$ $t^{\ltimes}(u, p)=\mu(u, p)$, tem-se que $s(v)=f(q)=f(\mu(u, p))=t(u)$, ou seja, $(v, u) \in \mathcal{G}_{1 s} \times{ }_{t} \mathcal{G}_{1}$ e $s(v \cdot u)=s(u)=f(p)$, ou seja, $(v \cdot u, p) \in \mathcal{G}_{1_{s}} \times{ }_{f} M$;

- $\mathbb{1}^{\ltimes}$ está bem definida, pois dado $p \in M$, tem-se que $s\left(1_{f(p)}\right)=f(p)$, ou seja, $\left(1_{f(p)}, p\right) \in$ $\mathcal{G}_{1 s} \times{ }_{f} M$

- $i^{\ltimes}$ está bem definido, pois dado $(u, p) \in \mathcal{G}_{1_{s}} \times{ }_{f} M$, tem-se que $s\left(u^{-1}\right)=t(u)=$ $f(\mu(u, p))$, ou seja, $\left(u^{-1}, \mu(u, p)\right) \in \mathcal{G}_{1_{s}}{ }_{f} M$.

A seguir será mostrado que as operações descritas satisfazem a propriedades algébrica necessárias para que $\mathcal{G} \ltimes M$ seja um grupóide.

- (Associatividade da multiplicação) Sejam $(u, p),(v, q),(w, r) \in \mathcal{G}_{1}{ }_{s} \times{ }_{f} M$, tais que $s^{\ltimes}(w, r)=t^{\ltimes}(v, q)$ e $s^{\ltimes}(v, q)=t^{\ltimes}(u, p)$, ou seja, $r=\mu(v, q)$ e $q=\mu(u, p)$. Então

$$
\begin{aligned}
& s^{\ltimes}((v, q) \cdot(u, p))=s^{\ltimes}(v \cdot u, p)=p=s^{\ltimes}(u, p), \\
& t^{\ltimes}((v, q) \cdot(u, p))=t^{\ltimes}(v \cdot u, p)=\mu(v \cdot u, p)=\mu(v, \mu(u, p))=\mu(v, q)=t^{\ltimes}(v, q) .
\end{aligned}
$$

Ainda

$$
((w, r) \cdot(v, q)) \cdot(u, p)=((w \cdot v) \cdot u, p)=(w \cdot(v \cdot u), p)=(w, r) \cdot((v, q) \cdot(u, p)) .
$$


- (Elemento neutro da multiplicação) Seja $p \in M$. Então

$$
s^{\ltimes}\left(1_{p}\right)=s^{\ltimes}\left(1_{f(p)}, p\right)=p, \quad t^{\ltimes}\left(1_{p}\right)=t^{\ltimes}\left(1_{f(p)}, p\right)=\mu\left(1_{f(p)}, p\right)=p .
$$

Ainda, dado $(u, p) \in \mathcal{G}_{1_{s}} \times_{f} M$, tem-se que

$$
\begin{aligned}
& (u, p) \cdot 1_{s^{\ltimes}(u, p)}=(u, p) \cdot 1_{p}=(u, p) \cdot\left(1_{f(p)}, p\right)=\left(u \cdot 1_{s(u)}, p\right)=(u, p), \\
& 1_{t^{\ltimes}(u, p)} \cdot(u, p)=1_{\mu(u, p)} \cdot(u, p)=\left(1_{f(\mu(u, p))}, \mu(u, p)\right) \cdot(u, p)=\left(1_{t(u)} \cdot u, p\right)=(u, p) .
\end{aligned}
$$

- (Elemento neutro da inversão) Seja $(u, p) \in \mathcal{G}_{1_{s}} \times_{f} M$. Então

$$
\begin{aligned}
& s^{\ltimes}\left((u, p)^{-1}\right)=\operatorname{pr}_{M}\left(u^{-1}, \mu(u, p)\right)=\mu(u, p)=t^{\ltimes}(u, p), \\
& t^{\ltimes}\left((u, p)^{-1}\right)=\mu\left(u^{-1}, \mu(u, p)\right)=\mu\left(1_{s(u)}, p\right)=\mu\left(1_{f(p)}, p\right)=p=s^{\ltimes}(u, p) .
\end{aligned}
$$

Ainda

$$
\begin{aligned}
& (u, p) \cdot(u, p)^{-1}=\left(u \cdot u^{-1}, \mu(u, p)\right)=\left(1_{f(\mu(u, p))}, \mu(u, p)\right)=1_{\mu(u, p)}=1_{t^{\ltimes}(u, p)}, \\
& (u, p)^{-1} \cdot(u, p)=\left(u^{-1} \cdot u, p\right)=\left(1_{s(u)}, p\right)=\left(1_{f(p)}, p\right)=1_{p}=1_{s^{\ltimes}}(u, p) .
\end{aligned}
$$

Proposição) Se $\mathcal{G}$ é um grupóide de Lie e $f$ e $\mu$ são suaves, então $\mathcal{G} \ltimes M$ é um grupóide de Lie e $\mu$ é uma submersão.

Demonstração. Observe, primeiramente, que a exigência da suavidade das aplicações estruturais está bem posta, dado que $s$ é uma submersão sobrejetora e, portanto, $\mathcal{G}_{1}{ }_{s} \times{ }_{f} M \subset$ $\mathcal{G}_{1} \times M$ é uma subvariedade mergulhada.

Ainda, as projeções canônicas $\operatorname{pr}_{\mathcal{G}_{1}}: \mathcal{G}_{1_{s}} \times{ }_{f} M \longrightarrow \mathcal{G}_{1}$ e $\operatorname{pr}_{M}: \mathcal{G}_{1{ }_{s}} \times_{f} M \longrightarrow M$ são suaves e, portanto, é imediato ver que $\mathrm{m}^{\ltimes}, \mathbb{1}^{\ltimes}$ e $i^{\ltimes}$ são suaves e $s^{\ltimes}$ é uma submersão sobrejetora e, consequentemente, $\mu:=t^{\ltimes}$ é uma submersão sobrejetora.

O grupóide $\mathcal{G} \ltimes M$ será dito o grupóide de ação de $\mathcal{G}$ por $M$, relativo a ação $\mu$ ao longo do mapa momento $f$.

Vale ressaltar, que dado $p \in M$,

- o grupo de isotropia de $\mathcal{G} \ltimes M$, em $p$, é o grupo de isotropia, em $p$, da ação de $\mathcal{G}_{f(p)}$ sobre $f^{-1}(\{f(p)\})$;

- as órbitas do grupóide de ação são dadas por

$$
(\mathcal{G} \ltimes M)(p)=t^{\ltimes}\left(\left(s^{\ltimes}\right)^{-1}(\{p\})\right)=\left\{\mu(u, p): u \in \mathcal{G}_{1} \text { e } s(u)=f(p)\right\}=\operatorname{Im}\left(\mu^{p}\right),
$$

ou seja, as órbitas do grupóide $\mathcal{G} \ltimes M$ são as "órbitas" da ação de $\mathcal{G}$ sobre $M$.

Quando $M=E$ é um fibrado vetorial sobre $\mathcal{G}_{0}, f=\pi$ é a projeção desse fibrado e $\mu_{u}$ : $E_{s(u)} \longrightarrow E_{t(u)}$ é um isomorfismo linear, a ação $\mu$ dará uma origem a uma representação de $\mathcal{G}$ 
$\underline{\text { em } E}$, ou seja, um morfismo de grupóides $\rho^{\mu}: \mathcal{G} \longrightarrow \mathbb{G} L(E)$, a saber, dado por $\rho_{1}^{\mu}(u):=\mu_{u} \mathrm{e}$ $\overline{\rho_{0}^{\mu}=}$ Id. Neste caso o grupóide $\mathcal{G} \ltimes E$ será dito o grupóide de transformação da representação $\rho^{\mu}$.

Cabe observar que, em contrapartida, uma representação $\rho: \mathcal{G} \longrightarrow \mathbb{G L}(E)$ sempre dará origem a uma ação $\mu^{\rho}: \mathcal{G}_{1 s_{s}}{ }_{\pi} E \longrightarrow E$, tal que $\mu_{u}^{\rho}: E_{s(u)} \longrightarrow E_{t(u)}$ é um isomorfismo linear, para todo $u \in \mathcal{G}_{1}$, de modo que ações "lineares" de grupóides em fibrados vetoriais e representações estão biunivocamente relacionadas.

Exemplo) (Representação de holonomia) Seja $M$ uma variedade diferenciável, $E$ um fibrado vetorial sobre $M$, com projeção $\pi$ e $\mathrm{H} \subset \mathrm{T} E$ uma distribuição horizontal ao longo de uma distribuição suave $D \subset \mathrm{T} M$.

Considere $\mathrm{Hol}^{\mathrm{H}}=\mathcal{P}^{\mathrm{H}} \rightrightarrows M$ o grupóide de holonomia de $\mathrm{H}$, onde $\mathcal{P}^{\mathrm{H}}$ é o conjunto dos operadores de transporte paralelo (com relação a $\mathrm{H}$ ) ao longo de curvas em $M$ suaves por partes e tangentes a $D$ (ver definição precisa na Seção 3.2).

Defina $\mu: \mathcal{P}^{\mathrm{H}}{ }_{s} \times{ }_{\pi} E \longrightarrow E$ por $\mu\left(\mathrm{P}_{\alpha}, x\right):=\mathrm{P}_{\alpha}(x)$. Então $\mu$ dá origem a uma representação $\rho: \mathrm{Hol}^{\mathrm{H}} \longrightarrow \mathbb{G} \mathbb{L}(E)$. De fato

- $\pi\left(\mu\left(\mathrm{P}_{\alpha}, x\right)\right)=\alpha(1)=t(\alpha)$, para todo $\left(\mathrm{P}_{\alpha}, x\right) \in \mathcal{P}^{\mathrm{H}}{ }_{s} \times{ }_{\pi} E$,

- $\mu\left(\operatorname{Id}_{E_{\pi(x)}}, x\right)=x$, para todo $x \in E$,

- $\mu\left(\mathrm{P}_{\beta}, \mu\left(\mathrm{P}_{\alpha}, x\right)\right)=\mu\left(\mathrm{P}_{\beta} \circ \mathrm{P}_{\alpha}, x\right)$, para todo $\left(\mathrm{P}_{\alpha}, x\right) \in \mathcal{P}^{\mathrm{H}}{ }_{s} \times_{\pi} E$ e $\mathrm{P}_{\beta} \in \mathcal{P}^{\mathrm{H}}$, tais que $s\left(\mathrm{P}_{\beta}\right)=t\left(\mathrm{P}_{\alpha}\right)$,

- $\mu_{\mathrm{P}_{\alpha}}=\mathrm{P}_{\alpha}$ é um isomorfismo, para todo $\mathrm{P}_{\alpha} \in \mathcal{P}^{\mathrm{H}}$.

A representação $\mu$ será dita a representação de holonomia da distribuição horizontal $H$.

Quando $D$ é integrável a uma folheação (regular) $\mathcal{F}$, vale ressaltar que, para cada $x \in E$,

- o grupo de isotropia, em $x$, do grupóide $\mathrm{Hol}^{\mathrm{H}} \ltimes E$ é o grupo de isotropia, em $x$, da ação de $\mathrm{Hol}_{\pi(x)}^{\mathrm{H}}$ sobre $E_{\pi(x)}$;

- a órbita, passando por $x$, do grupóide $\mathrm{Hol}^{\mathrm{H}} \ltimes E$ é a folha de holonomia da folheção de holonomia $\mathcal{F}^{\mathrm{H}}$ em $E$ (ver definição no exemplo de folheação de holonomia na seção 3.2 ).

\subsubsection{Ação de grupos sobre grupóides}

Sejam $\mathcal{G}$ um grupóide e $G$ um grupo. Uma ação à direita de $G$ sobre $\mathcal{G}$ é um par de ações à direita de $G$ sobre $\mathcal{G}_{0}$ e $\mathcal{G}_{1}$, tais que as operações $s, t, \mathbb{1}, \mathrm{m}$ e $i$ sejam $G$-equivariantes, ou seja, para todo,

- $s(u g)=s(u) g$ e $t(u g)=t(u) g$, para todo $g \in G$ e $u \in \mathcal{G}_{1}$, 
- $1_{x g}=1_{x} g$, para todo $g \in G$ e $x \in \mathcal{G}_{0}$,

- $(v g) \cdot(u g)=(v \cdot u) g$, para todo $g \in G$ e $u, v \in \mathcal{G}_{1}$, tal que $s(v)=t(u)$,

- $(u g)^{-1}=u^{-1} g$, para todo $g \in G$ e $u \in \mathcal{G}_{1}$.

Uma idéia natural é tentar construir um grupóide cujos objetos sejam as órbitas da ação de $G$ sobre $\mathcal{G}_{0}$ e as flechas sejam as órbitas da ação de $G$ sobre $\mathcal{G}_{1}$. De fato, tal idéia é factível, mas para a construção deste grupóide fazem-se necessárias algumas considerações.

Defina, primeiramente,

- ("source" e "target") $\bar{s}, \bar{t}: \mathcal{G}_{1} / G \longrightarrow \mathcal{G}_{0} / G$, por

$$
\bar{s}(G(u)):=G(s(u)), \quad \bar{t}(G(u)):=G(t(u)) ;
$$

- (seção identidade) $\overline{\mathbb{1}}: \mathcal{G}_{0} / G \longrightarrow \mathcal{G}_{1} / G$, por

$$
\overline{\mathbb{1}}(G(x)):=G\left(1_{x}\right)
$$

- (inversão) $\bar{i}: \mathcal{G}_{1} / G \longrightarrow \mathcal{G}_{1} / G$, por

$$
\bar{i}(G(u)):=G\left(u^{-1}\right) .
$$

Observe que $\bar{s}, \bar{t}, \overline{\mathbb{1}}$ e $\bar{i}$ estão bem definidas devido a $G$-equivariância de $s, t, \mathbb{1}$ e $i$, respectivamente. Seguindo a simplificação de notação adotada no início do capítulo, os elementos $\overline{\mathbb{1}}(G(u))$ e $\bar{i}(G(u))$ serão, simplesmente, denotados por $1_{G(u)}$ e $G(u)^{-1}$.

Note que, para que a ação de $G$ sobre $\mathcal{G}$ gere um grupóide nos espaços de órbitas, ainda se faz necessário definir uma operação de multiplicação entre as órbitas da ação de $G$ sobre $\mathcal{G}_{1}$.

Uma primeira idéia, que talvez seja natural, para se definir uma tal multiplicação $\overline{\mathrm{m}}$, seria considerar que $\overline{\mathrm{m}}(G(v), G(u))=G(v \cdot u)$. Mas isto somente seria possível se $s(v)=t(u)$, para todo $u, v \in \mathcal{G}_{1}$, tal que $G(v)=G(u)$, o que não é necessariamente verdadeiro. Mas esta idéia pode ser corrigida, com o uso do Lema 3.2, quando a ação de $G$ sobre $\mathcal{G}$ for livre (conceito definido logo por meio do lema a seguir).

Lema 3.1) A ação de $G$ sobre $\mathcal{G}_{0}$ é livre se, e somente se, a ação de $G$ sobre $\mathcal{G}_{1}$ é livre.

Demonstração. Suponha que a ação de $G$ sobre $\mathcal{G}_{0}$ é livre. Então dados $u \in \mathcal{G}_{1}$ e $g \in G$, tais que $u g=u$, tem-se que $s(u) g=s(u g)=s(u)$ e, portanto, $g=e$. Assim a ação de $G$ sobre $\mathcal{G}_{1}$ é livre.

Suponha que a ação de $G$ sobre $\mathcal{G}_{1}$ é livre. Então dados $x \in \mathcal{G}_{0}$ e $g \in G$, tai que $x g=x$, tem-se que $1_{x} g=1_{x g}=1_{x}$ e, portanto, $g=e$. Assim a ação de $G$ sobre $\mathcal{G}_{0}$ é livre.

Uma ação suave (à direita) de um grupo $G$ sobre um grupóide $\mathcal{G}$, em decorrência do lema anterior, será dita livre se, e somente se, a ação de $G$ sobre $\mathcal{G}_{1}$ ou sobre $\mathcal{G}_{0}$ for livre. 
Lema 3.2) Se a ação de $G$ sobre $\mathcal{G}$ for livre, então dados $u, v \in \mathcal{G}_{1}$, tais que $\bar{s}(G(v))=\bar{t}(G(u))$, existe um único $\theta_{v, u} \in G$, tal que $s(v) \theta_{v, u}=t(u)$.

Demonstração. Uma vez que $G(s(v))=\bar{s}(G(v))=\bar{t}(G(u))=G(t(u))$, existe $\theta_{v, u} \in G$, tal que $s(v) \theta_{v, u}=t(u)$. Suponha que exista $\tilde{\theta}_{v, u} \in G$, tal que $s(v) \tilde{\theta}_{v, u}=t(u)$. Neste caso, uma vez que a ação de $G$ sobre $\mathcal{G}_{0}$ é livre, tem-se que $\tilde{\theta}_{v, u} \theta_{v, u}^{-1} \in G_{s(v)}=\{e\}$ e, portanto, $\theta_{v, u}=\tilde{\theta}_{v, u}$.

Defina, finalmente,

- (multiplicação) $\overline{\mathrm{m}}:\left(\mathcal{G}_{1} / G\right)_{\bar{s}} \times_{\bar{t}}\left(\mathcal{G}_{1} / G\right) \longrightarrow\left(\mathcal{G}_{1} / G\right)$, por

$$
\overline{\mathrm{m}}(G(v), G(u))=G\left(v \theta_{v, u} \cdot u\right)
$$

Seguindo a simplificação de notação adotada no início do capítulo, o elemento $\overline{\mathrm{m}}(G(v), G(u))$ será, simplesmente, denotado por $G(v) \cdot G(u)$.

Antes de mostrar que $\overline{\mathrm{m}}$ está bem definida e que $\mathcal{G}_{1} / G \rightrightarrows \mathcal{G}_{0} / G$ é um grupóide é necessária a exposição de algumas propriedades algébricas dos elementos $\theta_{v, u}$. Com este propósito, por questões de conveniência, defina $U:=\left\{(u, v) \in \mathcal{G}_{1} \times \mathcal{G}_{1}: \bar{s}(G(v))=\bar{t}(G(u))\right\}$ e $\theta: U \subset$ $\mathcal{G}_{1} \times \mathcal{G}_{1} \longrightarrow G$ por $\theta(v, u):=\theta_{v, u}$.

\section{Lema) (Propriedades algébricas de $\theta$ )}

a) $\theta(v, u g)=\theta(v, u) g \quad$ e $\theta(v g, u)=g^{-1} \theta(v, u)$, para todo $(v, u) \in U$ e $g \in G$,

b) $\theta\left(u, 1_{s(u)}\right)=e=\theta\left(1_{t(u)}, u\right)$, para todo $u \in \mathcal{G}_{1}$,

c) $\theta(w \theta(w, v) \cdot v, u)=\theta(v, u)$ e $\theta(w, v \theta(v, u) \cdot u)=\theta(w, v) \theta(v, u)$, para todo $(w, v),(v, u) \in$ $U$

d) $\theta\left(u, u^{-1}\right)=e=\theta\left(u^{-1}, u\right)$, para todo $u \in \mathcal{G}_{1}$.

\section{Demonstração.}

a) $\operatorname{Sejam}(v, u) \in U$ e $g \in G$. Então

$$
t(u)=t(u g) g^{-1}=s(v) \theta(v, u g) g^{-1}, \quad t(u)=s(v g) \theta(v g, u)=s(v) g \theta(v g, u) .
$$

Assim, pela unicidade de $\theta_{v, u}$, segue que $\theta(v, u)=\theta(v, u g) g^{-1}$ e $\theta(v, u)=g \theta(v g, u)$, ou seja,

$$
\theta(v, u g)=\theta(v, u) g, \quad \theta(v g, u)=g^{-1} \theta(v, u) .
$$

b) Seja $u \in \mathcal{G}_{1}$. Então

$$
s(u)=t\left(1_{s(u)}\right)=s(u) \theta\left(u, 1_{s(u)}\right), \quad t(u)=s\left(1_{t(u)}\right) \theta\left(1_{t(u)}, u\right)=t(u) \theta\left(1_{t(u)}, u\right) .
$$


Assim, como a ação de $G$ é livre, segue que

$$
\theta\left(u, 1_{s(u)}\right)=e=\theta\left(1_{t(u)}, u\right)
$$

c) $\operatorname{Sejam}(w, v),(v, u) \in U$. Então

$$
\begin{aligned}
t(v) \theta(v, u) & =t(v \theta(v, u))=t(v \theta(v, u) \cdot u)=s(w) \theta(w, v \theta(v, u) \cdot u) \\
t(u) & =s(w \theta(w, v) \cdot v) \theta(w \theta(w, v) \cdot v, u)=s(v) \theta(w \theta(w, v) \cdot v, u) .
\end{aligned}
$$

Assim, pela unicidade de $\theta(w, v)$ e $\theta(v, u)$, respectivamente, segue que

$$
\theta(w, v) \theta(v, u)=\theta(w, v \theta(v, u) \cdot u), \quad \theta(v, u)=\theta(w \theta(w, v) \cdot v, u) .
$$

d) Seja $u \in \mathcal{G}_{1}$. Então

$$
s(u)=t\left(u^{-1}\right)=s(u) \theta\left(u, u^{-1}\right), \quad t(u)=s\left(u^{-1}\right) \theta\left(u^{-1}, u\right)=t(u) \theta\left(u^{-1}, u\right) .
$$

Assim, como a ação de $G$ é livre, segue que

$$
\theta\left(u, u^{-1}\right)=e=\theta\left(u^{-1}, u\right)
$$

Lema) Se a ação de $G$ sobre $\mathcal{G}$ for livre, então $\mathcal{G} / G:=\mathcal{G}_{1} / G \rightrightarrows \mathcal{G}_{0} / G$, com as operações definidas, é um grupóide.

Demonstração. Observe, primeiramente, que a operação de multiplicação está bem definida, pois se $(G(v), G(u))=(G(\tilde{v}), G(\tilde{u})) \in \overline{\mathcal{G}}_{1_{\bar{s}}} \times_{\bar{t}} \overline{\mathcal{G}}_{1}$, então $u g=\tilde{u}$ e $v h=\tilde{v}$ e, portanto, $\theta_{v, u} g=h \theta_{\tilde{v}, \tilde{v}}$ o que, pela $G$-equivariância de $\mathrm{m}$, implica que $\mathrm{m}\left(v \theta_{v, u}, u\right) g=\mathrm{m}\left(v \theta_{v, u} g, u g\right)=$ $\mathrm{m}\left(\tilde{v} \theta_{\tilde{v}, \tilde{u}}, \tilde{u}\right)$.

A seguir será mostrado que as operações descritas satisfazem a propriedades algébrica necessárias para que $\mathcal{G} / G$ seja um grupóide.

- (Associatividade da multiplicação) Sejam $u, v, w \in \mathcal{G}_{1}$, tal que $(v, u),(w, v) \in \overline{\mathcal{G}}_{1 \bar{s}_{\bar{s}}} \overline{\overline{\mathcal{G}}}_{1}$. Então

$$
\begin{aligned}
& \bar{s}(G(v) \cdot G(u))=\bar{s}\left(G\left(v \theta_{v, u} \cdot u\right)\right)=G\left(s\left(v \theta_{v, u} \cdot u\right)\right)=G(s(u))=\bar{s}(G(u)), \\
& \bar{t}(G(v) \cdot G(u))=\bar{t}\left(G\left(v \theta_{v, u} \cdot u\right)\right)=G\left(t\left(v \theta_{v, u} \cdot u\right)\right)=G\left(t(v) \theta_{v, u}\right)=\bar{t}(G(v)) .
\end{aligned}
$$

Ainda

$$
\begin{aligned}
G(w) \cdot(G(v) \cdot G(u)) & =G(w) \cdot G\left(v \theta_{v, u} \cdot u\right) \\
& =G\left(w \theta\left(w, v \theta_{v, u} \cdot u\right) \cdot\left(v \theta_{v, u} \cdot u\right)\right) \\
& =G\left(\left(w \theta\left(w, v \theta_{v, u} \cdot u\right) \cdot v \theta_{v, u}\right) \cdot u\right) .
\end{aligned}
$$


Logo, pelo item (c) do lema anterior, tem-se que

$$
G(w) \cdot(G(v) \cdot G(u))=G\left(\left(w \theta_{w, v} \theta_{v, u} \cdot v \theta_{v, u}\right) \cdot u\right)=G\left(\left(w \theta_{w, v} \cdot v\right) \theta_{v, u} \cdot u\right)
$$

Assim, pelo item (c) do lema anterior, segue que

$$
\begin{aligned}
G(w) \cdot(G(v) \cdot G(u)) & =G\left(\left(w \theta_{w, v} \cdot v\right) \theta\left(w \theta_{w, v} \cdot v, u\right) \cdot u\right) \\
& =G\left(w \theta_{w, v} \cdot v\right) \cdot G(u) \\
& =(G(w) \cdot G(v)) \cdot G(u) .
\end{aligned}
$$

- (Elemento neutro da multiplicação) Sejam $x \in \mathcal{G}_{0}$ e $u \in \mathcal{G}_{1}$. Então

$$
\bar{s}\left(1_{G(x)}\right)=G\left(s\left(1_{x}\right)\right)=G(x)=G\left(t\left(1_{x}\right)\right)=\bar{t}\left(1_{G(x)}\right) .
$$

Ainda

$$
\begin{aligned}
& G(u) \cdot 1_{\bar{s}(G(u))}=G(u) \cdot G\left(1_{s(u)}\right)=G\left(u \theta\left(u, 1_{s(u)}\right) \cdot 1_{s(u)}\right)=G\left(u \cdot 1_{s(u)}\right)=G(u), \\
& 1_{\bar{t}(G(u))} \cdot G(u)=G\left(1_{t(u)}\right) \cdot G(u)=G\left(1_{t(u)} \theta\left(1_{t(u)}, u\right) \cdot u\right)=G\left(1_{t(u)} \cdot u\right)=G(u) .
\end{aligned}
$$

- (Elemento neutro da inversão) Seja $u \in \mathcal{G}_{1}$. Então

$$
\begin{aligned}
& \bar{s}\left(G(u)^{-1}\right)=G\left(s\left(u^{-1}\right)\right)=G(t(u))=\bar{t}(G(u)), \\
& \bar{t}\left(G(u)^{-1}\right)=G\left(t\left(u^{-1}\right)\right)=G(s(u))=\bar{s}(G(u)) .
\end{aligned}
$$

Ainda

$$
\begin{aligned}
& G(u)^{-1} \cdot G(u)=G\left(u^{-1} \theta_{u^{-1}, u} \cdot u\right)=G\left(u^{-1} \cdot u\right)=1_{\bar{s}(G(u))}, \\
& G(u) \cdot G(u)^{-1}=G\left(u \theta_{u, u^{-1}} \cdot u^{-1}\right)=G\left(u \cdot u^{-1}\right)=1_{\bar{t}(G(u))} .
\end{aligned}
$$

O grupóide $\mathcal{G} / G$ será dito o grupóide quociente de $\mathcal{G}$ pela ação (à direita) de $G$.

Lema) Seja $\mathcal{G}$ é um grupóide de Lie e $G$ é um grupo de Lie agindo suavemente (à direita) sobre $\mathcal{G}$. Então a ação de $G$ sobre $\mathcal{G}_{0}$ é própria se, e somente se, a ação de $G$ sobre $\mathcal{G}_{1}$ é própria.

\section{Demonstração.}

Suponha que a ação de $G$ sobre $\mathcal{G}_{0}$ é própria.

Sejam $\left(g_{n}\right)_{n \in \mathbb{N}} \subset G$ e $\left(u_{n}\right)_{n \in \mathbb{N}} \subset \mathcal{G}_{1}$ convergente, tais que $\left(u_{n} g_{n}\right)_{n \in \mathbb{N}}$ converge, com $u_{n} \rightarrow u$ e $u_{n} g_{n} \rightarrow v$. Então, uma vez que $s$ é suave (em particular, contínua), tem-se que $s\left(u_{n}\right) \rightarrow s(u)$ e $s\left(u_{n}\right) g_{n}=s\left(u_{n} g_{n}\right) \rightarrow s(v)$. Assim, pela Proposição 1.2, segue que $\left(g_{n}\right)_{n \in \mathbb{N}}$ tem subsequência convergente e, portanto, a ação de $G$ sobre $\mathcal{G}_{1}$ é própria.

Suponha que a ação de $G$ sobre $\mathcal{G}_{1}$ é própria. 
Sejam $\left(g_{n}\right)_{n \in \mathbb{N}} \subset G$ e $\left(x_{n}\right)_{n \in \mathbb{N}} \subset \mathcal{G}_{0}$ convergente, tal que $\left(x_{n} g_{n}\right)_{n \in \mathbb{N}}$ converge, com $x_{n} \rightarrow$ $x$ e $x_{n} g_{n} \rightarrow y$. Então, uma vez que $\mathbb{1}$ é suave (em particular, contínua), tem-se que $1_{x_{n}} \rightarrow$ $1_{x}$ e $1_{x_{n}} g_{n}=1_{x_{n} g_{n}} \rightarrow 1_{y}$. Assim, pela Proposição 1.2 , segue que $\left(g_{n}\right)_{n \in \mathbb{N}}$ tem subsequência convergente e, portanto, a ação de $G$ sobre $\mathcal{G}_{0}$ é própria.

Uma ação suave (à direita) de um grupo de Lie $G$ sobre um grupóide de Lie $\mathcal{G}$, em decorrência da proposição anterior, será dita própria se e somente se a ação de $G$ sobre $\mathcal{G}_{1}$ ou sobre $\mathcal{G}_{0}$ for própria.

Proposição 3.3) Se $G$ é um grupo de Lie, $\mathcal{G}$ é um grupóide de Lie e a ação (à direita) de $G$ sobre $\mathcal{G}$ é suave, livre e própria, então $\mathcal{G} / G$ é um grupóide de Lie.

Demonstração. Observe, primeiramente, que as propriedades da ação implicam que $\mathcal{G}_{1} / G$ e $\mathcal{G}_{0} / G$ são variedades e as aplicações quociente $\pi_{1}: \mathcal{G}_{1} \longrightarrow \mathcal{G}_{1} / G$ e $\pi_{0}: \mathcal{G}_{0} \longrightarrow \mathcal{G}_{0} / G$ são submersões sobrejetoras.

A suavidade de $\bar{s}, \bar{t}, \overline{\mathbb{1}}$ e $\bar{i}$ segue do fato de que $\bar{s} \circ \pi_{1}=\pi_{0} \circ s, \bar{t} \circ \pi_{1}=\pi_{0} \circ t, \bar{i} \circ \pi_{1}=\pi_{0} \circ i \mathrm{e}$ $\overline{\mathbb{1}} \circ \pi_{0}=\pi_{1} \circ \mathbb{1}$ (ver os diagramas a seguir). Ainda, dado que $s$ e $t$ são submersões sobrejetoras, segue que $\bar{s}$ e $\bar{t}$ são submersões sobrejetoras.
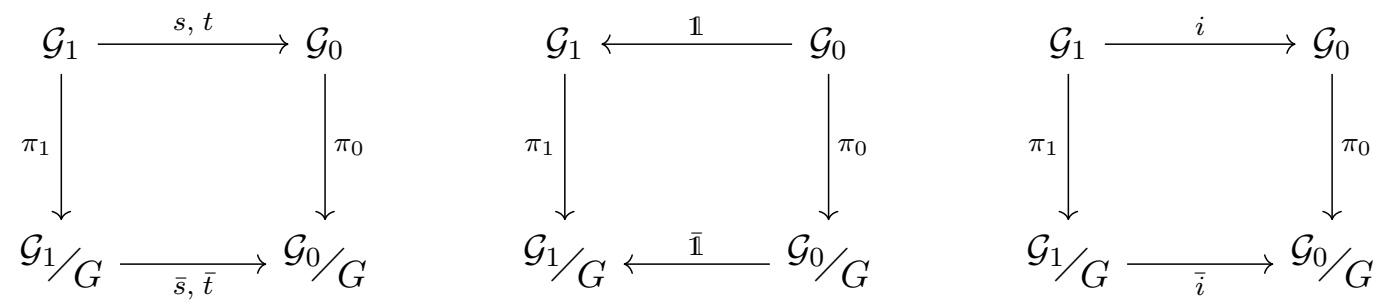

Enfim, como $\pi_{1} \times \pi_{1}: \mathcal{G}_{1 s} \times{ }_{t} \mathcal{G}_{1} \longrightarrow \mathcal{G}_{1} / G_{\bar{s}} \times{ }_{\bar{t}} \mathcal{G}_{1} / G$, dada por $\pi_{1{ }_{s} \times{ }_{t}} \pi_{1}(v, u)=(G(v), G(u))$ é uma submersão e $\overline{\mathrm{m}} \circ\left(\pi_{1}{ }_{s}{ }_{t} \pi_{1}\right)=\pi_{1} \circ \mathrm{m}$ (ver o diagrama a seguir), segue que $\overline{\mathrm{m}}$ é suave.

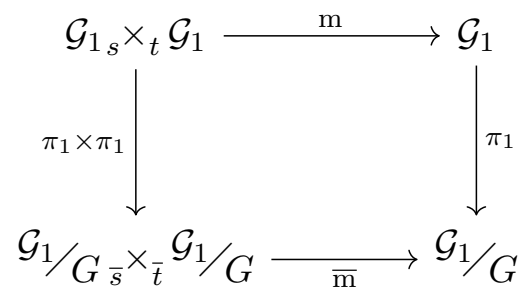

A seção será encerrada mostrando-se que um morfismo de grupóides $G$-invariante induz um morfismo de grupóides quocientes. 


\section{Capítulo 4.}

\section{Descrição Semi-local de uma FRS}

O Capítulo 4 será inteiramente dedicado à apresentação e demonstração dos principais resultados deste texto, os Teoremas A, B e C apresentados a seguir (mais a frente no texto, os Teoremas 4.12, 4.19 e 4.24) dentro de uma breve exposição do conteúdo de cada seção deste capítulo.

A Seção 4.1 apresentará a construção, em uma variedade Riemanniana com uma FRS $\mathcal{F}$, de distribuições (regulares) semi-locais (i.e. definidas em uma vizinhança tubular distinguida em torno de uma subvariedade $B$ fechada, saturada e contida em um estrato) intrínsecas a folheação $\mathcal{F}$. Uma delas, denotada por $\mathcal{K}$, será dada pelo plano tangente as fibras da vizinhança tubular distinguida, enquanto a outra, denotada por $\mathcal{N}$, será dada pela complementação ortogonal destes planos tangentes, considerando-se nas fibras a métrica plana induzida do espaço normal a $B$, por meio da aplicação exponencial.

Além disso, também será construída, a partir de uma extensão $\mathcal{T} \subset \mathrm{T} \mathcal{F}$ de $\mathrm{T} \mathcal{F}_{B}$, uma distribuição horizontal $\mathcal{T}^{\ell}$ ao longo de $\mathrm{TF}_{B}$, que dará origem a uma conexão parcial invariante por homotetias, permitindo derivar objetos ao longo das folhas, ao menos semi-localmente.

A Seção 4.2 prosseguirá com os passos da demonstração do Teorema A, o qual permitirá a conclusão de que a folheação de holonomia, obtida por meio da conexão construída na Seção 4.1, é dada por órbitas de um grupóide de Lie, quando a folheação induzida em $B$ for densa, o que ocorre, por exemplo, quando $B$ é o fecho de uma folha ou quando $B$ é o estrato mínimo da folheação.

Teorema A. Sejam $M$ uma variedade, $\mathcal{F}_{M}$ uma folheação (regular) densa de $M, E$ um fibrado vetorial Euclideano sobre $M$ e $\nabla$ uma conexão parcial linear métrica ao longo de $\mathrm{T} \mathcal{F}_{M}$, cujo grupóide de holonomia será denotado por Hol.

Então $\{\operatorname{Hol}(x)\}_{x \in E}$ é uma folheação singular, dada por órbitas de um grupóide de Lie.

A Seção 4.3 será iniciada com uma breve exposição de uma importante propriedade da distribuição $\mathcal{N}$, que permitirá o controle da dimensão dos subgrupos de isometrias das fibras da vizinhança tubular distinguida, que fixam a folheação infinitesimal, fato crucial à prova do Teorema B. Por fim, a seção será encerrada com o "passo à passo" da demonstração do Teorema B. Cabe ressaltar que parte significativa desta demonstração é similar e, em alguns momentos, até mesmo análoga a demonstração do Teorema A, de modo que a ordem e a disposição dos lemas que compõe a demonstração do Teorema B é, na medida do possível, a mesma utilizada na demonstração do Teorema A. 
Teorema B. Sejam $M$ uma variedade Riemanniana, $\mathcal{F}$ uma FRS e $B \subset M$ uma subvariedade fechada, saturada e contida em um estrato.

Então, existe $W$ uma vizinhança tubular saturada, em torno de $B$, tal que $\mathcal{F}^{\ell}$ e $\mathcal{F}^{\text {lc }}$ são dadas por órbitas de um grupóide de Lie.

A Seção 4.4 será iniciada com a apresentação da folheação de holonomia num contexto mais geral (o qual expõe-se brevemente a seguir), para em seguida exibir o enunciado preciso e uma prova do Teorema $\mathrm{C}$ que, essencialmente, mostra que a aplicação exponencial, definida em um aberto em torno da seção nula do fibrado normal de $B$, leva esta generalização da folheação de holonomia (resp. sua linearização e seu fecho local) na folheação $\mathcal{F}$ (resp. na folheação linearizada $\mathcal{F}^{\ell}$ e na folheação de fecho local $\mathcal{F}^{\text {lc }}$ ) restrita a uma vizinhança tubular saturada ou, mais sucintamente, mostra que a aplicação exponencial é uma difeomorfismo folheado.

\section{Modelo semi-local) (Folheação de holonomia generalizada) Sejam}

- $B$ uma variedade Riemanniana, com $\mathcal{F}_{B}$ uma folheação Riemanniana (regular) de $B$,

- $E$ um fibrado vetorial Euclideano sobre $B$, com projeção $\pi$,

- $\widetilde{\mathrm{H}} \subset \mathrm{T} E$ uma distribuição horizontal e $\mathrm{H} \subset \widetilde{\mathrm{H}}$ uma distribuição horizontal ao longo de $\mathrm{T} \mathcal{F}_{B}$, tal que a conexão parcial linear induzida por $\mathrm{H}$ seja métrica e cujo grupóide de holonomia será denotado por Hol;

- $\mathcal{F}^{E}=\left\{L_{x}^{E}\right\}_{x \in E}$ uma folheação Hol-invariante de $E$, tal que $E_{b}$ é saturada e $\mathcal{F}_{b}:=\mathcal{F}_{E_{b}}$ é uma FRS infinitesimal, para todo $b \in B$.

Considere, para cada $b \in B, \mathrm{~K}_{b}^{0} \subset \mathrm{Iso}\left(E_{b}\right)$ o maior subgrupo de Lie conexo que fixa $\mathcal{F}_{b}$.

Então

- $\mathcal{F}\left(\mathrm{H}, \mathcal{F}^{E}\right)=\left\{\operatorname{Hol}\left(L_{x}^{E}\right)\right\}_{x \in E}$

- $\mathcal{F}^{\ell}\left(\mathrm{H}, \mathcal{F}^{E}\right)=\left\{\operatorname{Hol}\left(\mathrm{K}_{\pi(x)}^{0}(x)\right)\right\}_{x \in E}$;

- $\mathcal{F}^{\mathrm{lc}}\left(\mathrm{H}, \mathcal{F}^{E}\right)=\left\{\operatorname{Hol}\left(\overline{\mathrm{K}}_{\pi(x)}^{0}(x)\right)\right\}_{x \in E} ;$

são folheações singulares de $E$ (ver figura 4.1 a seguir).

Teorema C. Sejam $M$ uma variedade Riemanniana, $\mathcal{F}$ uma FRS e $B \subset M$ uma subvariedade fechada, saturada e contida em um estrato.

Então, existem $W$ uma $\varepsilon$-VTD, em torno de $B$, e $\mathcal{T} \subset \mathrm{T} \mathcal{F}_{W}$ uma extensão de $\mathrm{T} \mathcal{F}_{B}$, tal que $\mathcal{F}_{W}, \mathcal{F}^{\ell}$ e $\mathcal{F}^{\text {lc }}$ são isomorfas a $\mathcal{F}\left(\mathcal{T}^{\ell}, \mathcal{F}^{\nu B}\right), \mathcal{F}^{\ell}\left(\mathcal{T}^{\ell}, \mathcal{F}^{\nu B}\right)$ e $\mathcal{F}^{\text {lc }}\left(\mathcal{T}^{\ell}, \mathcal{F}^{\nu B}\right)$, respectivamente, onde $\mathcal{F}^{\nu B}$ é a folheação de $\nu B$ pelas folhas infinitesimais de $\mathcal{F}$. 

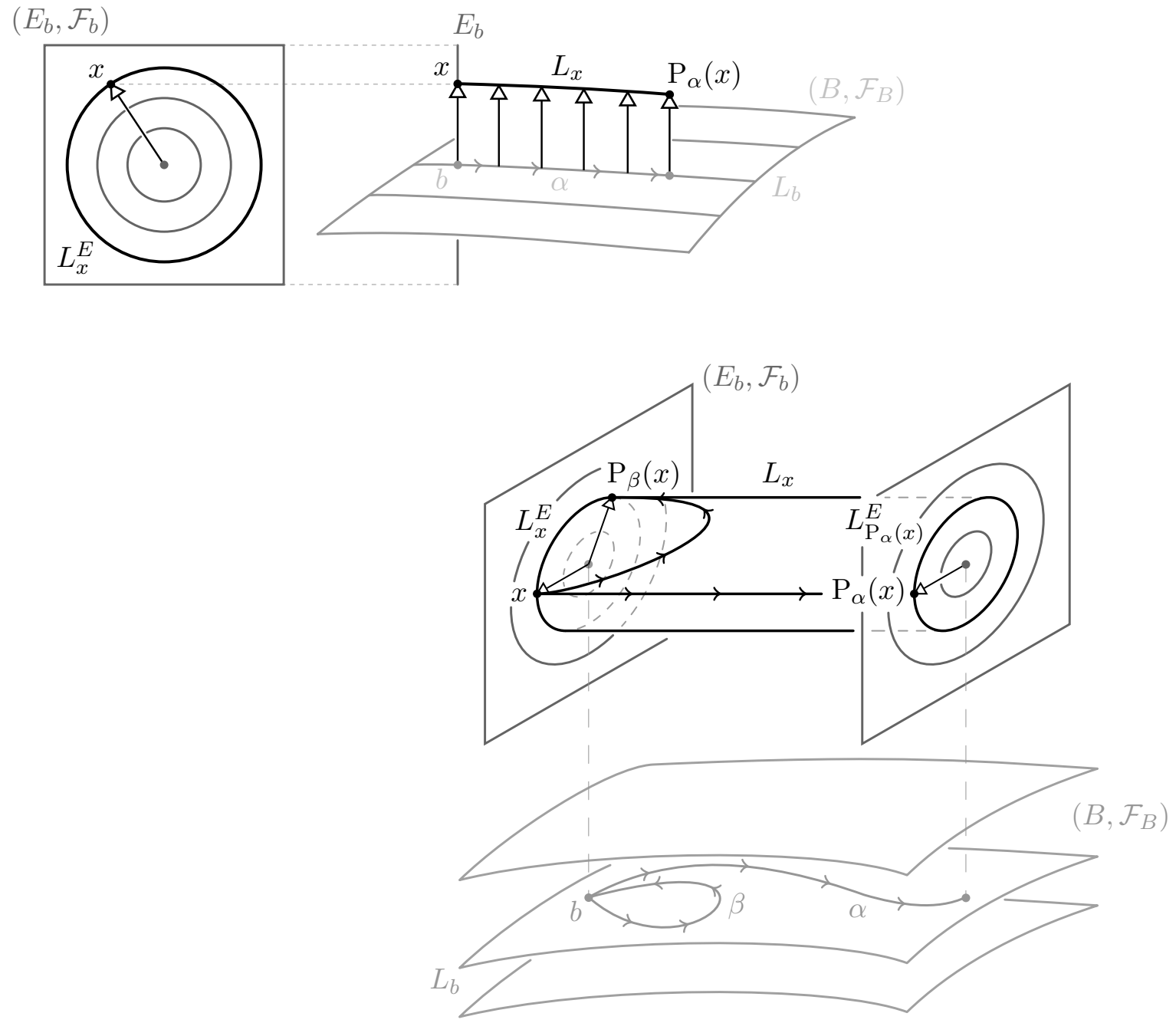

Figura 4.1 : Dois esquemas possíveis para ilustrar a partição $\mathcal{F}\left(\mathrm{H}, \mathcal{F}^{E}\right)$, onde $L_{x}=\operatorname{Hol}\left(L_{x}^{E}\right)$.

\subsection{Distribuições semi-locais de uma FRS}

Sejam $(M, g)$ uma variedade Riemanniana, $\mathcal{F}$ uma FRS e $B \subset M$ subvariedade fechada, saturada e contida em um estrato, de modo que $B$ é uma união de folhas de mesma dimensão e a partição $\mathcal{F}_{B}$ é uma folheação (regular) de $B$.

Denote por $\nu B$ o fibrado normal a $B$ e por $\pi$ a sua projeção na base $B$. Ainda, para cada $b \in B$, denote por $\nu_{b} L_{b}$ e $\nu_{b} B$ os espaços normal a folha e normal a $B$, passando por $b$, respectivamente.

Dado $\varepsilon>0$, será denotado por $\nu^{\varepsilon} B \subset \nu B$ o subfibrado de bolas abertas de raio $\varepsilon$ em $\nu B$. Ainda, para cada $b \in B$, será denotado por $S_{b}^{\varepsilon}$ o "slice" normal de raio $\varepsilon$ passando por $b$, por $\nu_{b}^{\varepsilon} L_{b}$ a bola aberta de raio $\varepsilon$ em $\nu_{b} L_{b}$ e por $\nu_{b}^{\varepsilon} B$ a bola aberta de raio $\varepsilon$ em $\nu_{b} B$. Será considerado, 
em $S_{b}^{\varepsilon}$, a métrica plana $g_{b}$ induzida pela aplicação exponencial, ou seja,

$$
g_{b}:=\left.\left(\left.\exp _{b}\right|_{\nu_{b}^{\varepsilon} L_{b}}\right)_{*}\left(\left.g\right|_{b}\right)\right|_{\nu_{b}^{\varepsilon} L_{b}}
$$

onde $\left.g\right|_{b}: \mathrm{T}_{b} M \times \mathrm{T}_{b} M \longrightarrow \mathbb{R}$ é a métrica Riemannina de $M$ sobre $b$.

Ao longo desta seção, dada $W=\exp \left(\nu^{\varepsilon} B\right)$ uma $\varepsilon$-VTD, em torno de $B$, com projeção na base denotada por $\rho: W \longrightarrow B$, será denotado por $W_{b}$ a fibra de $W$ sobre $b \in B$, ou seja, $W_{b}=\exp _{b}\left(\nu_{b}^{\varepsilon} B\right)$. Observe que $\pi=\rho \circ \exp , \operatorname{em} \nu^{\varepsilon} B$.

Dada $W$ uma $\varepsilon$-VTD, em torno de $B$, defina a distribuição (regular)

$$
\mathcal{K}:=\operatorname{Ker}(\mathrm{d} \rho)
$$

Observe que $\mathcal{K}$ é invariante por homotetias, pois dado que $\rho \circ h_{\lambda}=\rho$, onde $h_{\lambda}$ é uma $\lambda$-homotetia, para todo $\lambda \in(0,1]$, tem-se que $\left.\left.\mathrm{d} \rho\right|_{h_{\lambda}(p)} \circ \mathrm{d} h_{\lambda}\right|_{p}=\left.\mathrm{d} \rho\right|_{p}$ e, portanto, $\left.\left.\mathrm{d} h_{\lambda}\right|_{p}\left(\left.\mathcal{K}\right|_{p}\right) \subset \mathcal{K}\right|_{h_{\lambda}(p)}$, para todo $p \in W$.

Uma vez que $W_{b} \subset S_{b}^{\varepsilon}$, para todo $b \in B$, tem-se que $\left.\mathcal{K}\right|_{p}=\mathrm{T}_{p} W_{\rho(p)}$ é um subespaço vetorial de $\mathrm{T}_{p} S_{\rho(p)}^{\varepsilon}$, para todo $p \in W$. Defina a distribuição (regular) $\mathcal{N} \subset \mathrm{T} W$ por

$$
\left.\mathcal{N}\right|_{p}:=\left(\left.\mathcal{K}\right|_{p}\right)^{\perp g_{\rho(p)}},
$$

ou seja, $\left.\mathcal{N}\right|_{p}$ é o complemento $g_{\rho(p)}$-ortogonal de $\left.\mathcal{K}\right|_{p}$ em $\mathrm{T}_{p} S_{\rho(p)}^{\varepsilon}$, para todo $p \in W$ (ver figura 4.3, mais adiante). Segue, diretamente desta definição, que $\mathcal{N}$ é uma distribuição (regular) suave, tal que $\left.\mathcal{N}\right|_{B} \subset \mathrm{T} B$, dado que $\left.\mathcal{N}\right|_{b}=\nu_{b} L_{b} \cap \mathrm{T}_{b} B$, para todo $b \in B$. Devido a isto, é possível considerar a linearização da distribuição $\mathcal{N}$, em torno de $B$ (conceito definido na seção 2.2).

A seguir, na Proposição 4.4, será mostrado que sempre é possível considerar $W$ uma $\varepsilon$-VTD em torno de $B$, na qual exista uma distribuição horizontal invariante por homotetias e tangente a $\mathcal{F}_{W}$, de modo que nesta vizinhança será sempre possível derivar na direção das folhas de $\mathcal{F}$.

A existência desta distribuição horizontal passa pela construção de uma $\varepsilon$-VTD $W$ e de uma distribuição (regular) $\mathcal{T} \subset \mathrm{T} W$ tangente a folheação $\mathcal{F}_{W}$ que extenda $\mathrm{T} \mathcal{F}_{B}$ (i.e. $\left.\mathcal{T}\right|_{B}=\mathrm{T} \mathcal{F}_{B}$ ) de modo que $\mathcal{T} \cap \mathcal{K}=\{0\}$.

A construção de $\mathcal{T}$, exposta na Proposição 4.3, será feita por meio dos Lemas 4.1 e 4.2, baseados no Lema 2.16 e nas Proposições 2.17 e 3.1 presentes em [1].

Lema 4.1) Existem $W$ uma $\varepsilon$-VTD, em torno de $B$, e $g_{0}$ uma métrica em $W$, tais que $\nu_{p}^{g_{0}} L_{p} \subset$ $\mathrm{T}_{p} S_{\rho(p)}^{\varepsilon}$, para todo $p \in W$.

Idéia da demonstração. Dado $b \in B$, considere $\varepsilon_{b}>0, \Omega_{b} \subset B$ um aberto relativamente compacto (em torno de $b$ ), $W_{b}:=\operatorname{Tub}_{\varepsilon_{b}}\left(\Omega_{b}\right), S_{b} \subset M$ um "slice" (passando por $b$ ) e $\pi_{b}: W_{b} \longrightarrow$ $S_{b} \cap W_{b}$ uma submersão sobrejetora, tal que

- $\left\{\pi_{b}^{-1}(p)\right\}_{p \in S_{b}}$ é uma subfolheação (regular) de $\mathcal{F}_{W_{b}}$,

- $\mathrm{T}_{q} W_{b}=\mathrm{T}_{q} S_{\rho(q)} \oplus \mathrm{T}_{q} \pi_{b}^{-1}\left(\pi_{b}(q)\right)$, para todo $q \in W_{b}$, 


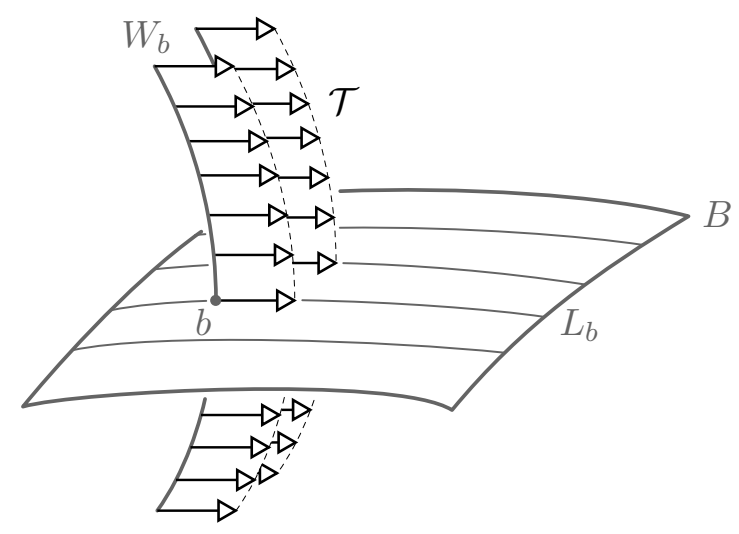

Figura 4.2: Esquema ilustrativo de como deve ser construída a distribuição (regular) $\mathcal{T}$ que extende $\mathrm{T} \mathcal{F}_{B}$

- $\left.\rho\right|_{\pi_{b}^{-1}(p)}: \pi_{b}^{-1}(p) \longrightarrow B$ é uma imersão, para todo $p \in S_{b}$.

Defina a métrica $g^{W_{b}}$, em $W_{b}$, por

$$
\left.g^{W_{b}}\right|_{p}:=\left.\left.\left.\left(\left.\exp _{b}\right|_{\exp _{b}^{-1}\left(S_{b}\right)}\right)_{*} g\right|_{b}\right|_{p} \oplus\left(\left.\rho\right|_{\pi^{-1}(p)}\right)^{*} g_{B}\right|_{p} .
$$

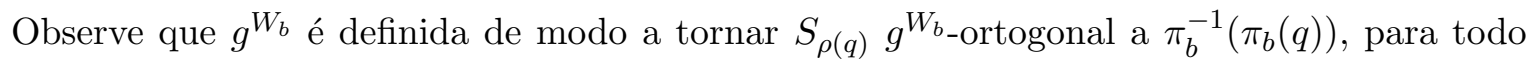
$q \in W_{b}$, e isto vem a implicar que

$$
\nu_{q}^{g^{W_{b}}} L_{q} \subset \nu_{q}^{g^{W_{b}}} \pi_{b}^{-1}\left(\pi_{b}(q)\right)=\mathrm{T}_{q} S_{\rho(q)}
$$

para todo $q \in W_{b}$, dado que $\left\{\pi_{b}^{-1}(p)\right\}_{p \in S_{b}}$ é uma subfolheação de $\mathcal{F}_{W_{b}}$.

Tome $\varepsilon>0,\left\{\varepsilon_{i}\right\}_{i \in I}$ e $\left\{\Omega_{i}\right\}_{i \in I}$, como descritos, tal que $\left\{W_{i}\right\}_{i \in I}$ seja uma cobertura aberta de $W:=\operatorname{Tub}_{\varepsilon}(B)$, onde $W_{i}:=\operatorname{Tub}_{\varepsilon_{i}}\left(\Omega_{i}\right)$, para cada $i \in I$ Considere $\left\{\rho_{i}\right\}_{i \in I}$ uma partição suave da unidade, subordinada à cobertura $\left\{W_{i}\right\}_{i \in I}$ e defina

$$
g^{0}:=\sum_{i \in I} \rho_{i} g^{W_{i}}
$$

Lema 4.2) Existem $W$ uma $\varepsilon$-VTD, em torno de $B$, e $\tilde{g}$ uma métrica em $W$, tal que $\left.\tilde{g}\right|_{B}=\left.g\right|_{B}$ e $\nu_{p}^{\tilde{g}} L_{p} \subset \mathrm{T}_{p} S_{\rho(p)}^{\varepsilon}$, para todo $p \in W$.

Idéia da demonstração. Seja $W$ e $g^{0}$ a $\varepsilon$-VTD e a métrica, obtidas no lema anterior. Considere

- $\mathrm{H} \subset \mathrm{T} W$ a distribuição dada por $\left.\mathrm{H}\right|_{p}:=\mathrm{T}_{p} S_{\rho(p)}$,

- $i:\left(\mathrm{H}^{\perp g^{0}}\right)^{\perp g} \longrightarrow \mathrm{T} W$ a inclusão de $\left(\mathrm{H}^{\perp g^{0}}\right)^{\perp g}$ em $\mathrm{T} W$.

Observe que, como $\left(\left.\mathrm{d} \rho\right|_{p}\right)$ restrita a $\left.\mathrm{H}\right|_{p} ^{\perp g_{p}^{0}}$ é injetora, para todo $p \in W$, tem-se que $\left.(\mathrm{d} \rho)^{*} g\right|_{B}$ é um produto interno em $\mathrm{H}^{\perp g^{0}}$. 
Defina, em $W$, a métrica $\tilde{g}$ por

$$
\tilde{g}:=\left.(\mathrm{d} \rho)^{*} g\right|_{B} \oplus i^{*} g
$$

Então $\left.\tilde{g}\right|_{B}=\left.g\right|_{B}$. De fato, para cada $b \in B$, como $\left.\nu_{b}^{g^{0}} L_{b} \subset \mathrm{H}\right|_{b}$, tem-se que $\left.\mathrm{H}\right|_{b} ^{\perp g_{b}^{0}}=$ $\nu_{b}^{g^{0}} S_{b} \subset \mathrm{T}_{b} L_{b}$. Mas $\operatorname{dim}\left(\left.\mathrm{H}\right|_{b} ^{\perp g_{b}^{0}}\right)=\operatorname{codim}\left(\left.\mathrm{H}\right|_{b}\right)=\operatorname{dim}\left(L_{b}\right)$ e, portanto, $\left.\mathrm{H}\right|_{b} ^{\perp g_{b}^{0}}=\mathrm{T}_{b} L_{b} . \operatorname{Logo}$, como d $\left.\rho\right|_{b}(u)=u$, para todo $u \in \mathrm{T}_{b} L_{b}$, conclui-se que $\left.\tilde{g}\right|_{B}=\left.g\right|_{B}$.

Vale ressaltar que os Lemas 4.1 e 4.2 são uma parte reduzida do conteúdo do Lema 2.16 e das Proposições 2.17 e 3.1 presentes em [1], onde afirma-se muito mais sobre as métricas $g^{0}$ e $\tilde{g}$. Este texto se restringirá as propriedades descritas, pois são as únicas necessárias à construção de $\mathcal{T}$ apresentada na proposição a seguir.

Proposição 4.3) Existem $W$ uma $\varepsilon$-VTD, em torno de $B$, e $\mathcal{T} \subset \mathrm{T} \mathcal{F}_{W}$ uma distribuição (regular) que extende $\mathrm{TF}_{B}$, tais que $\mathcal{T} \cap \mathcal{K}=\{0\}$.

Demonstração. Considere $W$ a $\varepsilon$-VTD, em torno de $B$, e $\tilde{g}$ a métrica em $W$, obtidas no lema anterior. Defina a distribuição (regular) $\mathcal{T} \subset \mathrm{T} W$ por

$$
\left.\mathcal{T}\right|_{p}:=\nu_{p}^{\tilde{g}} S_{\rho(p)}^{\varepsilon}
$$

Segue, das propriedades da métrica $\tilde{g}$ obtidas no lema anterior, que $\mathcal{T} \subset \mathrm{T} \mathcal{F}_{W}$. Dado que $\left.\mathcal{K}\right|_{p}=\mathrm{T}_{p} W_{\rho(p)} \subset \mathrm{T}_{p} S_{\rho(p)}^{\varepsilon}$, imediatamente, tem-se que $\left.\left.\mathcal{T}\right|_{p} \cap \mathcal{K}\right|_{p}=\left\{0_{p}\right\}$, para todo $p \in W$.

Por fim, como $\left.\mathcal{T}\right|_{b}=\nu_{b}^{\tilde{g}} S_{b}^{\varepsilon}=\nu_{b}^{g} S_{b}^{\varepsilon}=\mathrm{T}_{b} L_{b}$, para todo $b \in B$, segue que $\left.\mathcal{T}\right|_{B}=\mathrm{T} \mathcal{F}_{B}$.

Proposição 4.4) Seja $W$ uma $\varepsilon$-VTD, em torno de $B$, e $\mathcal{T} \subset \mathrm{T} \mathcal{F}_{W}$ uma distribuição (regular) que extende $\mathrm{T} \mathcal{F}_{B}$, tais que $\mathcal{T} \cap \mathcal{K}=\{0\}$. Então $\mathcal{T}^{\ell} \subset \mathrm{T} \mathcal{F}_{W}$ é uma distribuição horizontal, ao longo de $\mathrm{T} \mathcal{F}_{B}$, invariante por homotetias.

Demonstração. Observe, primeiramente, que é possível linearizar a distribuição (regular) $\mathcal{T}$, uma vez que $\left.\mathcal{T}\right|_{B}=\mathrm{TF}_{B} \subset \mathrm{T} B$.

Dado que $\mathcal{T}^{\ell}$ é $\rho$-invariante e $\left.\mathcal{T}^{\ell}\right|_{B}=\left.\mathcal{T}\right|_{B}$, segue que

$$
\left.\mathrm{d} \rho\right|_{p}\left(\left.\mathcal{T}^{\ell}\right|_{p}\right)=\left.\mathcal{T}^{\ell}\right|_{\rho(p)}=\left.\mathcal{T}\right|_{\rho(p)}=\mathrm{T}_{\rho(p)} L_{\rho(p)}
$$

ou seja, $\mathrm{d} \rho\left(\mathcal{T}^{\ell}\right)=\mathrm{T} \mathcal{F}_{B}$.

Além de determinar uma distribuição horizontal ao longo de $\mathrm{T} \mathcal{F}_{B}$, a escolha de uma distribuição (regular) $\mathcal{T}$, como descrita na proposição anterior, também determina uma distribuição horizontal $\mathrm{H}^{\mathcal{T}} \subset \mathrm{T} W$ invariante por homotetias, a saber, dada por $\mathrm{H}^{\mathcal{T}}:=\mathcal{N}^{\ell} \oplus \mathcal{T}^{\ell}$ (ver figura 4.3), como será mostrado na proposição seguinte. Mais adiante no texto (Teorema 4.24) ficará claro que estas distribuições horizontais fornecerão uma descrição semi-local da folheação linearizada $\mathcal{F}^{\ell}$ e da folheação de fecho local $\mathcal{F}^{\text {lc }}$. 


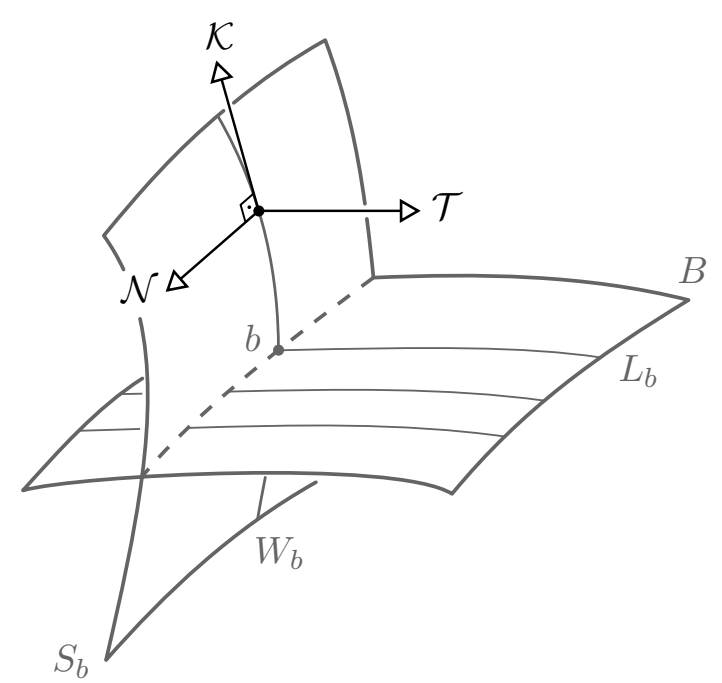

Figura 4.3 : Esquema ilustrativo da disposição e da interação entre as distribuições (regulares) $\mathcal{T}, \mathcal{K}$ e $\mathcal{N}$.

Proposição 4.5) Sejam $W$ uma $\varepsilon$-VTD, em torno de $B$, e $\mathcal{T} \subset \mathrm{T} \mathcal{F}_{W}$ uma distribuição (regular) que extende $\mathrm{T} \mathcal{F}_{B}$, tais que $\mathcal{T} \cap \mathcal{K}=\{0\}$. Então $\mathcal{N}^{\ell} \oplus \mathcal{T}^{\ell}$ é uma distribuição horizontal invariante por homotetias em $W$.

Demonstração. Seja $p \in W, \operatorname{com} \rho(p)=b$, e $\left.\left.v \in\left(\mathcal{K} \oplus \mathcal{N}^{\ell}\right)\right|_{p} \cap \mathcal{T}^{\ell}\right|_{p}$. Existem $\left.v_{\mathcal{K}} \in \mathcal{K}\right|_{p}$ e $\left.v_{\mathcal{N}} \in \mathcal{N}^{\ell}\right|_{p}$, tais que $v=v_{\mathcal{K}}+v_{\mathcal{N}}$, e existem $X_{\mathcal{T}} \in \mathfrak{X}(\mathcal{T})$ e $X_{\mathcal{N}} \in \mathfrak{X}(\mathcal{N})$, tais que $\left.X_{\mathcal{T}}^{\ell}\right|_{p}=v$ e $\left.X_{\mathcal{N}}^{\ell}\right|_{p}=v_{\mathcal{N}}$.

Dado que os campos linearizados são projetáveis, ou seja, $\rho$-invariantes (ver Proposição 2.14), tem-se que

$$
0=\left.\mathrm{d} \rho\right|_{p}\left(v_{\mathcal{K}}\right)=\left.\mathrm{d} \rho\right|_{p}\left(\left.X_{\mathcal{T}}^{\ell}\right|_{p}-\left.X_{\mathcal{N}}^{\ell}\right|_{p}\right)=\left.X_{\mathcal{T}}^{\ell}\right|_{b}-\left.X_{\mathcal{N}}^{\ell}\right|_{b}
$$

e, portanto,

$$
\left.X_{\mathcal{N}}^{\ell}\right|_{b}=\left.\left.X_{\mathcal{T}}^{\ell}\right|_{b} \in \mathcal{T}^{\ell}\right|_{b}=\mathrm{T}_{b} L_{b},\left.\left.\quad X_{\mathcal{N}}^{\ell}\right|_{b} \in \mathcal{N}^{\ell}\right|_{b}=\nu_{b} L_{b},
$$

ou seja,

$$
\left.\mathrm{d} \rho\right|_{p}\left(v_{\mathcal{N}}\right)=\left.\mathrm{d} \rho\right|_{p}\left(\left.X_{\mathcal{N}}^{\ell}\right|_{p}\right)=\left.X_{\mathcal{N}}^{\ell}\right|_{b} \in \mathrm{T}_{b} L_{b} \cap \nu_{b} L_{b}=\{0\}
$$

Logo, como $\left.\operatorname{Ker}\left(\left.\mathrm{d} \rho\right|_{p}\right) \cap \mathcal{N}^{\ell}\right|_{p}=\{0\}$, segue que $v_{\mathcal{N}}=0$ e, consequentemente, $v=v_{\mathcal{K}}$. Entretanto

$\left.\left.\mathcal{T}^{\ell}\right|_{p} \cap \mathcal{K}\right|_{p}=\{0\}$, pois $\mathcal{T}^{\ell}$ é uma distribuição horizontal ao longo de $\mathrm{T} \mathcal{F}_{B}$. Conclui-se, então, que $v=0$.

$\left.\left.\operatorname{Assim}\left(\mathcal{K} \oplus \mathcal{N}^{\ell}\right)\right|_{p} \cap \mathcal{T}^{\ell}\right|_{p}=\{0\}$ e, como

$$
\operatorname{dim}(W)=\operatorname{dim}\left(S_{b}^{\varepsilon}\right)+\operatorname{dim}\left(L_{b}\right)=\operatorname{posto}\left(\mathcal{K} \oplus \mathcal{N}^{\ell}\right)+\operatorname{posto}\left(\mathcal{T}^{\ell}\right)
$$


tem-se que $\mathrm{T}_{p} W=\left.\left.\left(\mathcal{K} \oplus \mathcal{N}^{\ell}\right)\right|_{p} \oplus \mathcal{T}^{\ell}\right|_{p}$

A seção será encerrada com a observação de que a invariância por homotetias das distribuições $\mathcal{K}, \mathcal{N}^{\ell}$ e $\mathcal{T}^{\ell}$ permitirá transportá-las ao subfibrado aberto $\nu^{\varepsilon} B \subset \nu B$, por meio da aplicação exponencial, e extendê-las, por meio da homotetia natural de $\nu B$, à todo fibrado normal a $B$, de modo a obter distribuições (regulares) $\mathcal{K}_{\nu B}, \mathcal{N}_{\nu B}^{\ell}$ e $\mathcal{T}_{\nu B}^{\ell}$ em $\nu B$.

As propriedades e as relações entre estas distribuições, apresentadas neste capítulo, também são transportadas ao fibrado $\nu B$, de modo que

- $\mathcal{K}_{\nu B}=\operatorname{Ker}(\mathrm{d} \pi)$ é a distribuição vertical em $\nu B$, uma vez que $\pi=\rho \circ \exp$ em $\nu^{\varepsilon} B$;

- $\mathcal{K}_{\nu B}$ e $\mathcal{N}_{\nu B}^{\ell}$ são $g$-ortogonais, uma vez que a métrica $g_{b}$, para cada $b \in B$, utilizada para definir $\mathcal{N}$ é induzida pela aplicação exponencial.

- $\mathcal{T}_{\nu B}^{\ell} \subset \mathrm{T}(\nu B)$ é uma distribuição horizontal, ao longo de $\mathrm{T} \mathcal{F}_{B}$, bem como $\mathrm{H}_{\nu B}^{\mathcal{T}}:=$ $\mathcal{N}_{\nu B}^{\ell} \oplus \mathcal{T}_{\nu B}^{\ell}$ é uma distribuição horizontal.

Visando a simplicidade de notação, nas seções seguintes a esta, as distribuições $\mathcal{K}_{\nu B}, \mathcal{N}_{\nu B}^{\ell} \mathrm{e}$ $\mathcal{T}_{\nu B}^{\ell}$ serão, simplesmente, denotadas por $\mathcal{K}, \mathcal{N}^{\ell}$ e $\mathcal{T}^{\ell}$, respectivamente, por um abuso de notação e sem prejuízo à compreensão.

Enfim, vale ressaltar que tanto $\mathcal{K}$ quanto $\mathcal{N}^{\ell}$ são distribuições intrínsecas da folheação, na vizinhança $W$, enquanto $\mathcal{T}^{\ell}$ é uma distribuição extrínseca, no sentido de que ela advém de uma escolha, dentre muitas possíveis, de uma distribuição $\mathcal{T}$ tangente a folheação que extende $\mathrm{T} \mathcal{F}_{B}$ e tal que $\mathcal{T} \cap \mathcal{K}=\{0\}$.

\subsection{Subfolheações de holonomia semi-locais (Teorema A)}

Sejam $(M, g)$ uma variedade Riemanniana, $\mathcal{F}$ uma FRS e $B \subset M$ subvariedade fechada, saturada e contida em um estrato, de modo que $\mathcal{F}_{B}$ é uma folheação (regular) de $B$.

Considere $W=\exp \left(\nu^{\varepsilon} B\right)$ uma $\varepsilon$-VTD, com projeção $\rho: W \longrightarrow B$, e $\mathcal{T} \subset \mathrm{TF}_{W}$ uma distribuição (regular) que extenda $T \mathcal{F}_{B}$ e tal que $\mathcal{T} \cap \mathcal{K}=\{0\}$. Foi visto na seção anterior que $\mathcal{T}$ determinava uma distribuição horizontal $\mathcal{T}^{\ell} \subset \mathrm{T}(\nu B)$ ao longo de $\mathrm{T} \mathcal{F}_{B}$ que é tangente a $\mathcal{F}$.

Seguindo o exemplo de folheação de holonomia apresentado na seção 1.1.1, a distribuição $\mathcal{T}^{\ell}$ determina uma folheação singular $\mathcal{F}^{\mathcal{T}}$ em $\nu B$. A saber, a folha de $\mathcal{T}^{\ell}$-holonomia passando por $x \in \nu B$ é dada por

$$
L_{x}^{\mathcal{T}}=\left\{\mathrm{P}_{\alpha}(x) \in \nu B: \alpha \in C_{\text {par. }}^{\infty}\left([0,1], \mathcal{F}_{B}\right) \text { e } \alpha(0)=\pi(x)\right\}
$$

onde, para cada $\alpha \in C_{\text {par. }}^{\infty}\left([0,1], \mathcal{F}_{B}\right), \mathrm{P}_{\alpha}$ é o operador de transporte $\mathcal{T}^{\ell}$-paralelo ao longo de $\alpha$. Dado que $\mathcal{T}^{\ell}$ é uma linearização e também é tangente a $\mathcal{F}$, tem-se que

$$
\mathcal{F}^{\mathcal{T}} \leqslant \mathcal{F}^{\ell} \leqslant \mathcal{F}_{W}
$$


A presente seção será dedicada a mostrar que, quando $\mathcal{F}_{B}$ for densa (e.g. quando $B$ é o estrato mínimo ou o fecho de uma folha), $\mathcal{F}^{\mathcal{T}}$ será dada por órbitas de um grupóide de Lie. Isto seguirá como consequência do Teorema 4.12 apresentado na seção 4.2.2 (Teorema A apresentado no início deste capítulo).

\subsubsection{Construções preliminares à prova do Teorema $\mathrm{A}$}

Será apresentado, na seção 4.2.2, o enunciado preciso e a prova do Teorema A (descrito no início deste capítulo).

Antes de apresentá-los, serão enunciados nesta seção alguns lemas e proposições necessários à prova de tal teorema. O leitor que se sentir confortável poderá, simplesmente, ignorar a prova dos mesmos e, apenas, ler e compreender o enunciado de tais lemas e proposições. Isto não trará prejuízos futuros à compreensão de como os mesmo serão utilizados na prova do Teorema A, ou seja, nenhuma informação contida na prova de tais lemas e proposições, será diretamente utilizada na prova deste teorema.

A compreensão dos lemas e das proposições que precedem o Teorema A, no entanto, exige que se estabeleça alguma notação preliminar, o que será feito a seguir.

Sejam $M$ uma variedade, $\mathcal{F}_{M}$ uma folheação (regular) de $M, E$ um fibrado vetorial Euclideano sobre $M$ com projeção $\pi$ e $\mathrm{H} \subset \mathrm{T} E$ uma distribuição horizontal ao longo de $\mathrm{TF}_{M}$, tal que a conexão parcial linear induzida $\nabla: \mathfrak{X}\left(\mathcal{F}_{M}\right) \times \Gamma(E) \longrightarrow \Gamma(E)$ seja métrica e cujo grupóide de holonomia será denominado por Hol.

Considere $C_{\text {par. }}^{\infty}\left([0,1], \mathcal{F}_{M}\right)$ o conjunto das curvas suaves por partes contidas em folhas de $\mathcal{F}_{M}$ e, para cada $\alpha \in C_{\text {par. }}^{\infty}\left([0,1], \mathcal{F}_{M}\right)$, considere $\mathrm{P}_{\alpha}: E_{\alpha(0)} \longrightarrow E_{\alpha(1)}$ o operador de transporte paralelo. Observe que $\mathrm{P}_{\alpha}$ é uma isometria (linear), dado que $\nabla$ é métrica.

Considere $\mathbb{O}(E)$ o $\mathbb{O}(n)$-fibrado principal de referênciais ortogonais do fibrado $E$, ou seja, $\mathbb{O}(E)=\sqcup_{p \in M}$ Iso $\left(\mathbb{R}^{n}, E_{p}\right)$, onde Iso $\left(\mathbb{R}^{n}, E_{p}\right)$ é o conjunto da isometrias (lineares) de $\mathbb{R}^{n}$ em $E_{p}$, para cada $p \in M$. Sem o risco de confusão e com abuso de notação, será denotado por $\pi$ a projeção do fibrado $\mathbb{O}(E)$ na base $M$. Ainda, considere $\mathbb{O H} \subset \mathrm{T} \mathbb{O}(E)$ a distribuição horizontal ao longo de $\mathcal{T F}_{M}$ induzida por $\mathrm{H} \subset \mathrm{T} E$ (ver [33], por exemplo, para a construção de $\mathbb{O H}$ ). A saber, para cada $\xi \in \mathbb{O}(E)$,

$$
\left.\mathbb{O H}\right|_{\xi}=\operatorname{span}\left\{\left.\frac{\mathrm{d}}{\mathrm{d} t} \mathrm{P}_{\alpha}^{t} \circ \xi\right|_{t=0} \in \mathrm{T}_{\xi} \mathbb{O}(E): \alpha \in C_{\text {par. }}^{\infty}\left([0,1] ; \mathcal{F}_{M}\right)\right\} .
$$

Ainda, para cada $X \in \mathfrak{X}\left(\mathcal{F}_{M}\right)$, considere $\bar{X}$ o levantamento $\mathbb{O}$ H-horizontal de $X$, a saber, o único campo tangente a $\mathbb{O H}$, tal que $\mathrm{d} \pi(\bar{X})=X \circ \pi$. Denote por $\Phi^{\bar{X}}: D^{\bar{X}} \subset \mathbb{R} \times \mathbb{O}(E) \longrightarrow \mathbb{O}(E)$ o fluxo de $\bar{X}$.

Aplicando-se o Teorema de Sussmann (Teorema 1.12) ao subconjunto de campos vetoriais $\left\{\bar{X} \in \mathfrak{X}(\mathbb{O H}): X \in \mathfrak{X}\left(\mathcal{F}_{M}\right)\right\} \subset \mathfrak{X}(E)$, obtém-se uma folheação singular que será denotada por 
$\mathcal{F}^{\mathbb{O H}}$ e cuja folha passando por $\xi \in \mathbb{O}(E)$ é dada por

$$
L_{\xi}^{\mathbb{O H}}:=\left\{\Phi_{t}^{\bar{X}}(\xi) \in \mathbb{O}(E): n \in \mathbb{Z}_{+}^{*}, X \in \mathfrak{X}\left(\mathcal{F}_{M}\right)^{n}, t \in \mathbb{R}^{n}: D_{t}^{\bar{X}} \neq \emptyset\right\}
$$

e satisfaz a seguinte propriedade

$$
\left.\mathbb{O} \mathrm{H}\right|_{\xi}=\operatorname{span}\left\{\left.\bar{X}\right|_{\xi} \in \mathrm{T}_{\xi} \mathbb{O}(E): X \in \mathfrak{X}\left(\mathcal{F}_{M}\right)\right\} \subset \mathrm{T}_{\xi} L_{\xi}^{\mathbb{O H}} .
$$

Vale observar que, com esta folheação em $\mathbb{O}(E)$, para cada $u \in \mathbb{R}^{n}$, a aplicação $\pi_{u}: \mathbb{O}(E) \longrightarrow E$ dada por $\pi_{u}(\xi):=\xi(u)$ é uma submersão folheada, considerando-se $E$ folheada pela folheação de holonomia $\mathcal{F}^{\mathrm{H}}$, cuja folha passando por $x \in E$ é dada por

$$
L_{x}^{\mathrm{H}}=\left\{\mathrm{P}_{\alpha}(x) \in E: \alpha \in C_{\text {par. }}^{\infty}\left([0,1], \mathcal{F}_{M}\right): \alpha(0)=\pi(x)\right\}
$$

(ver seção 1.1.1 para maiores detalhes a cerca da folheação de holonomia). Em vista desta observação, a folheação $\mathcal{F}^{\mathbb{O H}}$ será dita o levantamento ortogonal da folheação $\mathcal{F}^{\mathbf{H}}$.

A seguir será provado na Proposição 4.8 que, quando $\mathcal{F}_{M}$ é densa, a folheação $\mathcal{F}^{\mathbb{O H}}$ é regular. Isto será feito por meio do Lema 4.6, que decompõe o plano tangente as $\mathcal{F}^{\mathbb{O H}}$-folhas em duas direções, a saber, a direção da distribuição $\mathbb{O H}$ e a direção tangente as órbitas de holonomia de $\mathrm{H}$, e também por meio do Lema 4.7, que mostra como a densidade de $\mathcal{F}_{M}$ controla a dimensão dos grupos de holonomia de $\mathrm{H}$.

\section{Lema 4.6)}

a) $\quad L_{\xi}^{\mathbb{O H}}=\left\{\mathrm{P}_{\alpha} \circ \xi \in \mathbb{O}(E): \alpha \in C_{\text {par. }}^{\infty}\left([0,1], \mathcal{F}_{M}\right): \alpha(0)=\pi(\xi)\right\}$, para todo $\xi \in \mathbb{O}(E)$,

b) $\pi$ é folheada,

c) $\mathrm{T}_{\xi} L_{\xi}^{\mathbb{O H}}=\left.\mathbb{O H}\right|_{\xi} \oplus \mathrm{T}_{\xi} \operatorname{Hol}_{\pi(\xi)}(\xi)$, para todo $\xi \in \mathbb{O}(E)$.

\section{Demonstração.}

a) Segue diretamente do fato de que, para cada $X \in \mathfrak{X}\left(\mathcal{F}_{M}\right), \Phi_{t}^{\bar{X}}(\xi)=\mathrm{P}_{\alpha_{\pi(\xi)}}^{t} \circ \xi$, onde $\alpha_{\pi(\xi)}$ é a curva integral de $X$ passando por $\pi(\xi)$, para todo $(t, \xi) \in D^{\bar{X}}$.

b) Segue diretamente do item anterior que $\pi$ é folheada, pois $\pi\left(\mathrm{P}_{\alpha} \circ \xi\right)=\alpha(1)$, para todo $\alpha \in C_{\text {par. }}^{\infty}\left([0,1], \mathcal{F}_{M}\right)$ e $\xi \in \mathbb{O}(E)$, tal que $\alpha(0)=\pi(\xi)$.

c) Observe que, para cada $\xi \in \mathbb{O}(E) \operatorname{com} \pi(\xi)=p$, tem-se que

- $\mathbb{O}\left(E_{p}\right) \cap L_{\xi}^{\mathbb{O H}}=\operatorname{Hol}_{p}(\xi)$,

- $\left.\mathbb{O H}\right|_{\xi} \cap \mathrm{T}_{\xi} \mathbb{O}\left(E_{p}\right)=\{0\}$, dado que $\mathbb{O H}$ é uma distribuição horizontal em $\mathbb{O}(E)$, de modo que resta demonstrar que $\mathrm{T}_{\xi} L_{\xi}^{\mathbb{O H}}=\left.\mathbb{O H}\right|_{\xi}+\mathrm{T}_{\xi}\left(\mathbb{O}\left(E_{p}\right) \cap L_{\xi}^{\mathbb{O H}}\right)$. 
Seja $v \in \mathrm{T}_{\xi} L_{\xi}^{\mathbb{O H}}$. Tome $\tilde{\alpha} \in C^{\infty}\left([0,1], L_{\xi}^{\mathbb{O H}}\right)$, tal que $\tilde{\alpha}(0)=\xi$ e $\tilde{\alpha}^{\prime}(0)=v$ e defina $\alpha \in C^{\infty}([0,1], M)$ por $\alpha:=\pi \circ \tilde{\alpha}$. Dado que $\pi$ é folheada e $\operatorname{Im}(\tilde{\alpha}) \subset L_{\xi}^{\mathbb{O H}}$, tem-se que $\alpha \in C_{\text {par. }}^{\infty}\left([0,1], \mathcal{F}_{M}\right)$.

Segue que

$$
\left.\mathrm{d} \pi\right|_{\xi}\left(\tilde{\alpha}^{\prime}(0)-\left.\frac{\mathrm{d}}{\mathrm{d} t} \mathrm{P}_{\alpha}^{t} \circ \xi\right|_{t=0}\right)=\left.\frac{\mathrm{d}}{\mathrm{d} t} \pi(\tilde{\alpha}(t))\right|_{t=0}-\left.\frac{\mathrm{d}}{\mathrm{d} t} \pi\left(\mathrm{P}_{\alpha}^{t} \circ \xi\right)\right|_{t=0}=0
$$

ou seja,

$$
\tilde{\alpha}^{\prime}(0)-\left.\frac{\mathrm{d}}{\mathrm{d} t} \mathrm{P}_{\alpha}^{t} \circ \xi\right|_{t=0} \in \operatorname{Ker}\left(\left.\mathrm{d} \pi\right|_{\xi}\right)=\mathrm{T}_{\xi} \mathbb{O}\left(E_{b}\right) .
$$

Logo, como $\left.\left.\frac{\mathrm{d}}{\mathrm{d} t} \mathrm{P}_{\alpha}^{t} \circ \xi\right|_{t=0} \in \mathbb{O} \mathrm{H}\right|_{\xi} \subset \mathrm{T}_{\xi} L_{\xi}^{\mathbb{O H}}$, conclui-se que

$$
\tilde{\alpha}^{\prime}(0)-\left.\frac{\mathrm{d}}{\mathrm{d} t} \mathrm{P}_{\alpha}^{t} \circ \xi\right|_{t=0} \in \mathrm{T}_{\xi}\left(\mathbb{O}\left(E_{b}\right) \cap L_{\xi}^{\mathbb{O H}}\right)
$$

Assim, dado que

$$
v=\left.\frac{\mathrm{d}}{\mathrm{d} t} \mathrm{P}_{\alpha}^{t} \circ \xi\right|_{t=0}+\left(\tilde{\alpha}^{\prime}(0)-\left.\frac{\mathrm{d}}{\mathrm{d} t} \mathrm{P}_{\alpha}^{t} \circ \xi\right|_{t=0}\right)
$$

segue que $\left.v \in \mathbb{O H}\right|_{\xi}+\mathrm{T}_{\xi}\left(\mathbb{O}\left(E_{b}\right) \cap L_{\xi}^{\mathbb{O H}}\right)$.

\section{Lema 4.7)}

a) A ação de $\operatorname{Hol}_{p}$ sobre $\mathbb{O}\left(E_{p}\right)$ dada por $\mathrm{P}_{\alpha} \cdot \xi:=\mathrm{P}_{\alpha} \circ \xi$ é livre, para todo $p \in M$. Em particular, $\operatorname{dim}\left(\operatorname{Hol}_{p}\right)=\operatorname{dim}\left(\operatorname{Hol}_{p}(\xi)\right)$, para todo $\xi \in \mathbb{O}(E), \operatorname{com} \pi(\xi)=p$.

b) Se $\mathcal{F}_{M}$ é densa, então $p \longmapsto \operatorname{dim}\left(\mathrm{Hol}_{p}\right)$ é constante em $M$.

\section{Demonstração.}

a) Uma vez que uma isometria de $E_{p}$ que fixa todos os pontos é, necessariamente, a identidade, segue que

$$
\bigcap_{x \in E_{p}}\left(\operatorname{Hol}_{p}\right)_{x} \subset \bigcap_{x \in E_{p}} \operatorname{Iso}\left(E_{p}\right)_{x}=\{\operatorname{Id}\}
$$

e, portanto, a ação de $\operatorname{Hol}_{p}$ sobre $E_{p}$ é efetiva.

Logo, a ação de $\operatorname{Hol}_{p}$ sobre $\mathbb{O}\left(E_{p}\right)$ é livre. De fato, dado $\xi \in \mathbb{O}\left(E_{p}\right)$ e $\mathrm{P}_{\alpha} \in\left(\operatorname{Hol}_{p}\right)_{\xi}$, tem-se que $\mathrm{P}_{\alpha} \circ \xi=\xi$ e, portanto,

$$
\mathrm{P}_{\alpha} \in \bigcap_{u \in \mathbb{R}^{n}}\left(\operatorname{Hol}_{p}\right)_{\xi(u)}=\bigcap_{x \in E_{p}}\left(\operatorname{Hol}_{p}\right)_{x}=\{\mathrm{Id}\}
$$

$\operatorname{Assim} \operatorname{Hol}_{p}=\operatorname{Hol}_{p} /\left(\operatorname{Hol}_{p}\right)_{\xi} \approx \operatorname{Hol}_{p}(\xi)$ e, portanto, $\operatorname{dim}\left(\operatorname{Hol}_{p}\right)=\operatorname{dim}\left(\operatorname{Hol}_{p}(\xi)\right)$ 
b) Seja $p \in M$ e tome $\xi_{0} \in \mathbb{O}(E)$, com $\pi\left(\xi_{0}\right)=p$. Dado que $\mathcal{F}^{\mathbb{O H}}$ é uma folheação singular, $\xi \longmapsto \operatorname{dim}\left(L_{\xi}^{\mathbb{O H}}\right)$ é semicontínua inferiormente e, portanto, existe $\mathcal{U} \subset \mathbb{O}\left(E_{p}\right)$ vizinhança aberta de $\xi_{0}$, tal que $\operatorname{dim}\left(L_{\zeta}^{\mathbb{O H}}\right) \geqslant \operatorname{dim}\left(L_{\xi_{0}}^{\mathbb{O H}}\right)$, para todo $\zeta \in \mathcal{U}$. Defina $U_{p}:=\pi(\mathcal{U})$ uma vizinhança aberta de $p$.

Uma vez que $\mathbb{O}\left(E_{\pi(\xi)}\right) \cap L_{\xi}^{\mathbb{O H}}=\operatorname{Hol}_{\pi(\xi)}(\xi)$, pelo item (c) do Lema 4.6 e pelo intem anterior, segue que $\operatorname{dim}\left(L_{\xi}^{\mathbb{O H}}\right)=\operatorname{dim}\left(\left.\mathbb{O H}\right|_{\xi}\right)+\operatorname{dim}\left(\operatorname{Hol}_{\pi(\xi)}\right)$, para todo $\xi \in \mathbb{O}(E)$ e, como H é uma distribuição regular, conclui-se que $\operatorname{dim}\left(\operatorname{Hol}_{\pi(\zeta)}\right) \geqslant \operatorname{dim}\left(\operatorname{Hol}_{\pi\left(\xi_{0}\right)}\right)$, para todo $\zeta \in \mathcal{U}$, ou seja,

$$
\operatorname{dim}\left(\operatorname{Hol}_{q}\right) \geqslant \operatorname{dim}\left(\operatorname{Hol}_{p}\right)
$$

para todo $q \in U_{p}$.

Analogamente, para cada $q \in U_{p}$, existe $U_{q} \subset U_{p}$ vizinhança aberta de $q$, tal que

$$
\operatorname{dim}\left(\operatorname{Hol}_{\tilde{q}}\right) \geqslant \operatorname{dim}\left(\operatorname{Hol}_{q}\right)
$$

para todo $\tilde{q} \in U_{q}$. Dado que $\mathcal{F}_{M}$ é densa, tem-se que $L_{p} \cap U_{q} \neq \emptyset$, para todo $q \in U_{p}$. Então, tomando-se $q_{0} \in L_{p} \cap U_{q}$, segue que

$$
\operatorname{dim}\left(\operatorname{Hol}_{p}\right)=\operatorname{dim}\left(\operatorname{Hol}_{q_{0}}\right) \geqslant \operatorname{dim}\left(\operatorname{Hol}_{q}\right) \geqslant \operatorname{dim}\left(\operatorname{Hol}_{p}\right)
$$

ou seja, $\operatorname{dim}\left(\operatorname{Hol}_{q}\right)=\operatorname{dim}\left(\operatorname{Hol}_{p}\right)$.

Assim, $p \longmapsto \operatorname{dim}\left(\mathrm{Hol}_{p}\right)$ é constante em cada elemento da cobertura aberta $\left\{U_{p}\right\}_{p \in M}$ de $M$ e, portanto, $p \longmapsto \operatorname{dim}\left(\operatorname{Hol}_{p}\right)$ é constante em $M$.

Proposição 4.8) Se $\mathcal{F}_{M}$ é densa, então $\mathcal{F}^{\mathbb{O H}}$ é regular.

Demonstração. Segue, pelo item (a) do Lema 4.7 e pelo item (c) do Lema 4.6, que

$$
\operatorname{dim}\left(L_{\xi}^{\mathbb{O H}}\right)=\operatorname{dim}\left(\left.\mathbb{O H}\right|_{\xi}\right)+\operatorname{dim}\left(\operatorname{Hol}_{\pi(\xi)}\right)
$$

para todo $\xi \in \mathbb{O}(E)$. Mas, pelo item (b) do Lema 4.7, tem-se que $\xi \longmapsto \operatorname{dim}\left(\operatorname{Hol}_{\pi(\xi)}\right)$ é constante.

$\operatorname{Assim} \xi \longmapsto \operatorname{dim}\left(L_{\xi}^{\mathbb{O H}}\right)$ é constante e, portanto, $\mathcal{F}^{\mathbb{O H}}$ é regular.

Sabendo-se que existem condições para que $\mathcal{F}^{\mathbb{O H}}$ seja regular, devido a proposição anterior, será assumida esta hipótese para o prosseguimento desta seção. A regularidade de $\mathcal{F}^{\mathbb{O H}}$ permite considerar $\operatorname{Mon}\left(\mathcal{F}^{\mathbb{O H}}\right):=\Pi_{1}\left(\mathcal{F}^{\mathbb{O H}}\right) \rightrightarrows \mathbb{O}(E)$ o grupóide de monodromia da folheação $\mathcal{F}^{\mathbb{O H}}$, cujo o conjunto das flechas $\Pi_{1}\left(\mathcal{F}^{\mathbb{O H}}\right)$ é dado pelas classes de curvas $\mathcal{F}^{\mathbb{O H}}$-homotópicas (ver seção 3.2 para definição precisa deste grupóide).

Considere $\mu_{0}: \mathbb{O}(E) \times \mathbb{O}(n) \longrightarrow \mathbb{O}(E)$ a ação (à direita) canônica de $\mathbb{O}(n)$ sobre $\mathbb{O}(E)$, a saber, dada por $\mu_{0}(\xi, T)=\xi \circ T$ e, para cada $T \in \mathbb{O}(n)$, considere $\mu_{0}^{T}: \mathbb{O}(E) \longrightarrow \mathbb{O}(E)$ o isomorfismo de fibrados dado por $\mu_{0}^{T}(\xi)=\mu_{0}(\xi, T)=\xi \circ T$. Observe que $\mu_{0}^{T}$ é $\mathcal{F}^{\mathbb{O H}}$-folheado, pois $\mu_{0}^{T}\left(\mathrm{P}_{\alpha} \circ \xi\right)=\mathrm{P}_{\alpha} \circ \mu_{0}^{T}(\xi)$, para todo $\xi \in \mathbb{O}(E)$. A fim de não sobrecarregar a notação, 
quando não houver necessidade de explicitar a ação $\mu_{0}$, será denotado $\xi \cdot T:=\mu_{0}^{T}(\xi)$ e, para cada $\tilde{\alpha} \in C^{\infty}([0,1], \mathbb{O}(E))$, será denotado $\tilde{\alpha} \cdot T:=\mu_{0}^{T} \circ \tilde{\alpha}$.

Defina $\mu_{1}: \Pi_{1}\left(\mathcal{F}^{\mathbb{O H}}\right) \times \mathbb{O}(n) \longrightarrow \Pi_{1}\left(\mathcal{F}^{\mathbb{O H}}\right)$ por

$$
\mu_{1}([\tilde{\alpha}], T):=[\tilde{\alpha} \cdot T]
$$

Lema 4.9) $\quad \mu_{1}$ é uma ação (à direita) suave.

Demonstração. Observe, primeiramente, que $\mu_{1}$ está bem definida. De fato

- $\tilde{\alpha} \cdot T \in C^{\infty}\left([0,1], \mathcal{F}^{\mathbb{O H}}\right)$, pois $\operatorname{Im}(\tilde{\alpha}) \subset L_{\tilde{\alpha}(0)}^{\mathbb{O H}}$ e $\mu_{0}$ é $\mathcal{F}^{\mathbb{O H}}$-folheada de modo que

$$
\operatorname{Im}(\tilde{\alpha} \cdot T)=\mu_{0}^{T}(\operatorname{Im}(\tilde{\alpha})) \subset \mu_{0}^{T}\left(L_{\tilde{\alpha}(0)}^{\mathbb{O H}}\right) \subset L_{\mu_{0} T(\tilde{\alpha}(0))}^{\mathbb{O H}},
$$

- se $[\tilde{\alpha}]=[\tilde{\beta}]$, então $[\tilde{\alpha} \cdot T]=[\tilde{\beta} \cdot T]$, pois neste caso $\tilde{\alpha} \sim \tilde{\beta}$ e, dado que $\mu_{0}^{T}$ é suave $(\mathrm{em}$ particular, contínua) e $\mathcal{F}^{\mathbb{O H}}$-folheada, tem-se que $\tilde{\alpha} \cdot T \sim \tilde{\beta} \cdot T$.

Dado que $\mu_{1}$ está bem definida, verifica-se que $\mu_{1}$ é uma ação, pois dado que $\mu_{0}$ é uma ação, tem-se que $\mu_{1}([\tilde{\alpha}], \mathrm{Id})=[\tilde{\alpha} \cdot \mathrm{Id}]=[\tilde{\alpha}] \mathrm{e}$

$$
\mu_{1}\left(\mu_{1}([\tilde{\alpha}], T), S\right)=[(\tilde{\alpha} \cdot T) \cdot S]=[\tilde{\alpha} \cdot(T \circ S)]=\mu_{1}([\tilde{\alpha}], T \circ S)
$$

para todo $[\tilde{\alpha}] \in \Pi_{1}\left(\mathcal{F}^{\mathbb{O H}}\right)$ e $T, S \in \mathbb{O}(n)$.

Enfim, para verificar a suavidade de $\mu_{1}$ em um ponto $\left[\tilde{\alpha}_{0}\right] \times T_{0} \in \Pi_{1}\left(\mathcal{F}^{\mathbb{O H}}\right) \times \mathbb{O}(n)$, será computado a expressão de $\mu_{1}$ em cartas de em torno de $\left[\tilde{\alpha}_{0}\right] \times T_{0}$.

Considere

- $A \subset \mathbb{O}(n)$ uma vizinhança aberta em torno de $T_{0}$,

- $\varphi_{i}: U_{i} \subset \mathbb{O}(E) \longrightarrow \widetilde{U}_{i} \times \widetilde{S}_{i} \subset \mathbb{R}^{n} \times \mathbb{R}^{k}$ cartas folheadas em torno de $\tilde{\alpha}(i)=\varphi_{i}^{-1}(0,0)$, com $i \in\{0,1\}$ (como descritas no exemplo de grupóide de monodromia apresentado na Seção 3.2),

- $\psi_{i}: V_{i} \subset \mathbb{O}(E) \longrightarrow \widetilde{V}_{i} \times \widehat{S}_{i} \subset \mathbb{R}^{n} \times \mathbb{R}^{k}$ cartas em torno de $\tilde{\alpha}(i) \cdot T=\psi_{i}^{-1}(0,0)$, tais que $U_{i} \cdot A \subset V_{i}$, com $i \in\{0,1\}$ (como descritas no exemplo de grupóide de monodromia apresentado na Seção 3.2)

- $\tilde{\varphi}_{\alpha}: \widetilde{S}_{0} \longrightarrow \widetilde{S}_{1}$ a aplicação dada por $\tilde{\varphi}_{\alpha}:=\left.\left.\varphi_{1}\right|_{\widetilde{S}_{1}} ^{-1} \circ \varphi_{\alpha} \circ \varphi\right|_{\widetilde{S}_{0}}$, onde $\varphi_{\alpha}$ é a aplicação de holonomia ao longo de $\alpha$ e identifica-se $\{0\} \times \widetilde{S}_{0} \approx \widetilde{S}_{0}$ e $\{0\} \times \widetilde{S}_{1} \approx \widetilde{S}_{1}$.

Denote por $\Phi: \widetilde{U}_{0} \times \widetilde{S}_{0} \times \widetilde{U}_{1} \subset \mathbb{R}^{n} \times \mathbb{R}^{k} \times \mathbb{R}^{n} \longrightarrow \Pi_{1}\left(\mathcal{F}^{\mathbb{O H}}\right)$ e $\Psi: \widetilde{V}_{0} \times \widehat{S}_{0} \times \widetilde{V}_{1} \subset \mathbb{R}^{n} \times \mathbb{R}^{k} \times \mathbb{R}^{n} \longrightarrow$ $\Pi_{1}\left(\mathcal{F}^{\mathbb{O H}}\right)$ as cartas, em torno de $[\tilde{\alpha}]$ e $[\tilde{\alpha}] \cdot T$, associadas a $\varphi_{0}, \varphi_{1}$ e $\psi_{0}, \psi_{1}$, respectivamente.

Então

$$
\begin{aligned}
& \Psi\left(\mu_{1}\left(\Phi\left(\tilde{x}_{0}, \tilde{y}_{0}, \tilde{x}_{1}\right), \widehat{T}\right)\right)= \\
& \quad=\left(\left(\psi_{0} \circ \mu_{0}\right)\left(\varphi_{0}^{-1}\left(\tilde{x}_{0}, \tilde{y}_{0}\right), \widehat{T}\right),\left(\operatorname{pr}_{n} \circ \psi_{1} \circ \mu_{0}\right)\left(\varphi_{1}^{-1}\left(\tilde{x}_{1}, \varphi_{\tilde{\alpha}}\left(\tilde{y}_{0}\right)\right), \widehat{T}\right)\right)
\end{aligned}
$$


para todo $\left(\tilde{x}_{0}, \tilde{y}_{0}, \tilde{x}_{1}\right) \in \widetilde{U}_{0} \times \widetilde{S}_{0} \times \widetilde{U}_{1}$ e $\widehat{T} \in A$, onde $\varphi_{\tilde{\alpha}}$ é a aplicação de holonomia ao longo de $\tilde{\alpha}$ e $\operatorname{pr}_{n}: \mathbb{R}^{n} \times \mathbb{R}^{k} \longrightarrow \mathbb{R}^{n}$ é a projeção canônica.

Assim $\mu_{1}$ é suave.

Proposição 4.10) O par de ações $\left(\mu_{0}, \mu_{1}\right)$ é uma ação (à direita) suave, livre e própria sobre $\operatorname{Mon}\left(\mathcal{F}^{\mathbb{O H}}\right)$ e $\operatorname{Mon}\left(\mathcal{F}^{\mathbb{O H}}\right) / \mathbb{O}(n)$ é um grupóide de Lie.

Demonstração. Dado que $\mu_{0}$ e $\mu_{1}$ são ações suaves, para mostrar que o par $\left(\mu_{0}, \mu_{1}\right)$ define uma ação suave $\mu$, basta provar que as operações do grupóide $\operatorname{Mon}\left(\mathcal{F}^{\mathbb{O H}}\right)$ são $\mathbb{O}(n)$-equivariantes, com respeito a $\mu_{0}$ e $\mu_{1}$.

- Dados $[\tilde{\alpha}] \in \Pi_{1}\left(\mathcal{F}^{\mathbb{O H}}\right)$ e $T \in \mathbb{O}(n)$, tem-se que

$s\left(\mu_{1}([\tilde{\alpha}], T)\right)=\mu_{0}^{T}(\tilde{\alpha}(0))=\mu_{0}(s([\tilde{\alpha}]), T), \quad t\left(\mu_{1}([\tilde{\alpha}], T)\right)=\mu_{0}^{T}(\tilde{\alpha}(1))=\mu_{0}(t([\tilde{\alpha}]), T)$.

- Dados $\xi \in \mathbb{O}(E)$ e $T \in \mathbb{O}(n)$, tem-se que

$$
1_{\mu_{0}(\xi, T)}=\left[c_{\mu_{0}(\xi, T)}\right]=\left[c_{\xi} \cdot T\right]=\mu_{1}\left(1_{\xi}, T\right),
$$

onde $c_{\xi}$ e $c_{\mu_{0}(\xi, T)}$ são as curvas constantes em $\xi$ e $\mu_{0}(\xi, T)$, respectivamente.

- Dados $[\tilde{\alpha}] \in \Pi_{1}\left(\mathcal{F}^{\mathbb{O H}}\right)$ e $T \in \mathbb{O}(n)$, tem-se que

$$
\mu_{1}([\tilde{\alpha}], T)^{-1}=\left[\left(\mu_{0}^{T} \circ \tilde{\alpha}\right)^{-1}\right]=\left[\mu_{0}^{T} \circ \tilde{\alpha}^{-1}\right]=\mu_{1}\left([\tilde{\alpha}]^{-1}, T\right) .
$$

- $\operatorname{Dados} T \in \mathbb{O}(n)$ e $[\tilde{\alpha}],[\tilde{\beta}] \in \Pi_{1}\left(\mathcal{F}^{\mathbb{O H}}\right)$, tais que $s([\tilde{\alpha}])=\tilde{\alpha}(0)=\tilde{\beta}(1)=t([\tilde{\beta}])$, tem-se que

$$
\mu_{1}([\tilde{\alpha}], T) \cdot \mu_{1}([\tilde{\beta}], T)=\left[\left(\mu_{0}^{T} \circ \tilde{\alpha}\right) *\left(\mu_{0}^{T} \circ \tilde{\beta}\right)\right]=\left[\mu_{0}^{T} \circ(\tilde{\alpha} * \tilde{\beta})\right]=\mu_{1}([\tilde{\alpha}] \cdot[\tilde{\beta}], T) .
$$

Ainda, $\mu$ é livre e própria. De fato, devido ao Lema 3.1, é imediato que $\mu$ é livre, dado que $\mu_{0}$ é livre. Ainda, como $\mathbb{O}(n)$ é compacto, tem-se que $\mu_{0}$ e $\mu_{1}$ são próprias, ou seja, $\mu$ é própria.

Enfim, da Proposição 3.3, conclui-se que $\operatorname{Mon}\left(\mathcal{F}^{\mathbb{O H}}\right) / \mathbb{O}(n)$ é um grupóide de Lie.

Defina $\mu: \Pi_{1}\left(\mathcal{F}^{\mathbb{O H}}\right) / \mathbb{O}(n)_{s} \times_{\pi} E \longrightarrow E$ por

$$
\mu([\tilde{\alpha}] \cdot \mathbb{O}(n), x):=\left(\tilde{\alpha}(1) \circ \tilde{\alpha}(0)^{-1}\right)(x),
$$

onde $\tilde{\alpha}(0)^{-1}: E_{\alpha(0)} \longrightarrow \mathbb{R}^{n}$ é a inversa da isometria $\tilde{\alpha}(0)$.

Intuitivamente, a aplicação $\mu$ consiste em tomar as coordenadas de $x$ na base $\tilde{\alpha}(0)$ e construir o vetor dado por essas mesmas coordenadas na base $\tilde{\alpha}(1)$.

Proposição 4.11) $\mu$ gera uma representação de $\Pi_{1}\left(\mathcal{F}^{\mathbb{O H}}\right) / \mathbb{O}(n)$ em $E$. 
Demonstração. Observe, primeiramente, que $\mu$ está bem definida, pois se $[\tilde{\alpha}] \cdot \mathbb{O}(n)=$ $[\tilde{\beta}] \cdot \mathbb{O}(n)$, então existe $T \in \mathbb{O}(n)$, tal que $\tilde{\alpha} \sim \mu_{0}^{T} \circ \tilde{\beta}$ e, portanto, $\tilde{\alpha}(1) \circ \tilde{\alpha}(0)^{-1}=(\tilde{\beta}(1) \circ T) \circ$ $\left(T^{-1} \circ \tilde{\beta}(0)^{-1}\right)=\tilde{\beta}(1) \circ \tilde{\beta}(0)^{-1}$.

Dado que $\mu$ está bem definida, verifica-se que $\mu$ é uma ação ao longo de $\pi$. De fato

- se $([\tilde{\alpha}] \cdot \mathbb{O}(n), x) \in \Pi_{1}\left(\mathcal{F}^{\mathbb{O H}}\right) / \mathbb{O}(n)_{s} \times_{\pi} E$, então

$$
\pi(\mu([\tilde{\alpha}] \cdot \mathbb{O}(n), x))=\pi\left(\left(\tilde{\alpha}(1) \circ \tilde{\alpha}(0)^{-1}\right)(x)\right)=\pi(\tilde{\alpha}(1))=t([\tilde{\alpha}] \cdot \mathbb{O}(n)) ;
$$

- se $x \in E$, então

$$
\mu\left(1_{\pi(x)}, x\right)=\mu\left(1_{\xi} \cdot \mathbb{O}(n), x\right)=\mu\left(\left[c_{\xi}\right] \cdot \mathbb{O}(n), x\right)=x,
$$

onde $\xi \in \mathbb{O}(E)$ é tal que $\pi(\xi)=\pi(x)$ e $c_{\xi}$ é a curva constante em $\xi$

- se $([\tilde{\alpha}] \cdot \mathbb{O}(n), x) \in \Pi_{1}\left(\mathcal{F}^{\mathbb{O H}}\right) / \mathbb{O}(n)_{s} \times_{\pi} E$ e $[\tilde{\beta}] \cdot \mathbb{O}(n) \in \Pi_{1}\left(\mathcal{F}^{\mathbb{O H}}\right) / \mathbb{O}(n)$, tal que $s([\tilde{\beta}]$. $\mathbb{O}(n))=\tilde{\beta}(0)=\tilde{\alpha}(1)=t([\tilde{\alpha}] \cdot \mathbb{O}(n))$, então

$$
\begin{aligned}
\mu([\tilde{\alpha}] \cdot \mathbb{O}(n), \mu([\tilde{\beta}] \cdot \mathbb{O}(n), x)) & =\left(\tilde{\alpha}(1) \circ \tilde{\alpha}(0)^{-1}\right)\left(\left(\tilde{\beta}(1) \circ \tilde{\beta}(0)^{-1}\right)(x)\right) \\
& =\left(\left(\tilde{\alpha}(1) \cdot\left(\tilde{\alpha}(0)^{-1} \circ \tilde{\beta}(1)\right)\right) \circ \tilde{\beta}(0)^{-1}\right)(x) \\
& =\mu\left(\left[\tilde{\alpha} \cdot\left(\tilde{\alpha}(0)^{-1} \circ \tilde{\beta}(1)\right) * \tilde{\beta}\right] \cdot \mathbb{O}(n), x\right) \\
& =\mu\left(\mathrm{m}\left([\tilde{\alpha}] \cdot\left(\tilde{\alpha}(0)^{-1} \circ \tilde{\beta}(1)\right),[\tilde{\beta}]\right) \cdot \mathbb{O}(n), x\right) .
\end{aligned}
$$

Mas

$$
\mathrm{m}([\tilde{\alpha}] \cdot \mathbb{O}(n),[\tilde{\beta}] \cdot \mathbb{O}(n))=\mathrm{m}([\tilde{\alpha}] \cdot \theta([\tilde{\alpha}],[\tilde{\beta}]),[\tilde{\beta}]) \cdot \mathbb{O}(n)
$$

onde $\theta([\tilde{\alpha}],[\tilde{\beta}])$ é o único elemento de $\mathbb{O}(n)$, tal que $s([\tilde{\alpha}]) \cdot \theta([\tilde{\alpha}],[\tilde{\beta}])=t([\tilde{\beta}])$. Entretanto, $s([\tilde{\alpha}]) \cdot\left(\tilde{\alpha}(0)^{-1} \circ \tilde{\beta}(1)\right)=t([\tilde{\beta}])$, ou seja,

$$
\mathrm{m}([\tilde{\alpha}] \cdot \mathbb{O}(n),[\tilde{\beta}] \cdot \mathbb{O}(n))=\mathrm{m}\left([\tilde{\alpha}] \cdot\left(\tilde{\alpha}(0)^{-1} \circ \tilde{\beta}(1)\right),[\tilde{\beta}]\right) \cdot \mathbb{O}(n) .
$$

Logo

$$
\mu([\tilde{\beta}] \cdot \mathbb{O}(n), \mu([\tilde{\alpha}] \cdot \mathbb{O}(n), \xi))=\mu(\mathrm{m}([\tilde{\alpha}] \cdot \mathbb{O}(n),[\tilde{\beta}] \cdot \mathbb{O}(n)), \xi) .
$$

Ainda, $\mu$ é suave. De fato, dado que $\mathrm{q} \times \mathrm{Id}: \Pi_{1}\left(\mathcal{F}^{\mathbb{O H}}\right)_{s} \times{ }_{\pi} E \longrightarrow \Pi_{1}\left(\mathcal{F}^{\mathbb{O H}}\right) / \mathbb{O}(n)_{s} \times{ }_{\pi} E$ é uma submersão, onde q é a aplicação quociente, basta verificar que $\tilde{\mu}: \Pi_{1}\left(\mathcal{F}^{\mathbb{O H}}\right)_{s} \times{ }_{\pi} E \longrightarrow$ $E$ dada por $\tilde{\mu}([\alpha], x):=\mu([\alpha] \cdot \mathbb{O}(n), x)$ é suave. Considere, então, as aplicações suaves $F$ : $\mathbb{O}(E)_{\pi} \times{ }_{\pi} E \longrightarrow \mathbb{R}^{n}$ e $G: \mathbb{O}(E) \times \mathbb{R}^{n} \longrightarrow E$ dadas por $F(\xi, x):=\xi^{-1}(x)$ e $G(\xi, u)=\xi(u)$. Então

$$
\tilde{\mu}([\alpha], x):=G(t([\alpha]), F(s([\alpha]), x)),
$$

para todo $([\alpha], x) \in \Pi_{1}\left(\mathcal{F}^{\mathbb{O H}}\right)_{s} \times{ }_{\pi} E$ e, portanto, $\tilde{\mu}$ é suave por ser composição de aplicações 
suaves.

Enfim, para cada $[\tilde{\alpha}] \cdot \mathbb{O}(n) \in \Pi_{1}\left(\mathcal{F}^{\mathbb{O H}}\right) / \mathbb{O}(n)$, é imediato ver que $\mu^{[\tilde{\alpha}] \cdot \mathbb{O}(n)}: E_{\alpha(0)} \longrightarrow E_{\alpha(1)}$ é linear, pois $\mu^{[\tilde{\alpha}] \cdot \mathbb{O}(n)}=\tilde{\alpha}(1) \circ \tilde{\alpha}(0)^{-1}$ é composta de aplicações lineares.

Assim, $\mu$ gera uma representação $\rho: \Pi_{1}\left(\mathcal{F}^{\mathbb{O H}}\right) / \mathbb{O}(n) \longrightarrow \mathbb{G L}(E)$, a saber, dada por $\rho([\tilde{\alpha}]$. $\mathbb{O}(n))=\tilde{\alpha}(1) \circ \tilde{\alpha}(0)^{-1}$.

A seção será encerrada com a observação de que, sendo $\mu$ uma representação, é possível considerar o grupóide de transformação $\left(\Pi_{1}\left(\mathcal{F}^{\mathbb{O H}}\right) / \mathbb{O}(n)\right) \ltimes E$ da representação $\mu$ (ver seção 3.3.1 para a definição precisa deste grupóide) e este é, precisamente, o grupóide cujas órbitas são as folhas da folheação de holonomia $\mathcal{F}^{\mathrm{H}}$, como será visto na seção a seguir.

\subsubsection{Teorema A}

Será apresentado, nesta breve seção, o enunciado preciso e a prova do Teorema A descrito na introdução e no início deste capítulo.

Teorema 4.12) Sejam $M$ uma variedade, $\mathcal{F}_{M}$ uma folheação (regular) densa de $M, E$ um fibrado vetorial Euclidiano sobre $M$ e $\mathrm{H}$ uma distribuição horizontal, ao longo de $\mathrm{TF}_{M}$, compatível com a métrica das fibras de $E$, com grupóide de holonomia denotado por Hol.

Então

$$
\operatorname{Hol}(x)=\left(\Pi_{1}\left(\mathcal{F}^{\mathbb{O H}}\right) / \mathbb{O}(n) \ltimes E\right)(x),
$$

para todo $x \in E$, ou seja, $\mathcal{F}^{\mathrm{H}}$ é dada pelas órbitas do grupóide de Lie $\left(\Pi_{1}\left(\mathcal{F}^{\mathbb{O H}}\right) / \mathbb{O}(n)\right) \ltimes E$.

\section{Demonstração.}

- Seja $y \in\left(\Pi_{1}\left(\mathcal{F}^{\mathbb{O H}}\right) / \mathbb{O}(n) \ltimes E\right)(x)$. Então, existe $\tilde{\alpha} \in C^{\infty}\left([0,1], \mathcal{F}^{\mathbb{O H}}\right)$, tal que

$$
y=\left(\tilde{\alpha}(1) \circ \tilde{\alpha}(0)^{-1}\right)(x) .
$$

Mas $\tilde{\alpha}(1) \in L_{\tilde{\alpha}(0)}^{\mathbb{O H}}$ e, portanto, existe $\alpha \in C_{\text {par. }}^{\infty}\left([0,1], \mathcal{F}_{M}\right)$, tal que $\tilde{\alpha}(1)=\mathrm{P}_{\alpha} \circ \tilde{\alpha}(0)$.

Logo

$$
y=\left(\mathrm{P}_{\alpha} \circ \tilde{\alpha}(0) \circ \tilde{\alpha}(0)^{-1}\right)(x)=\mathrm{P}_{\alpha}(x) \in L_{x}^{\mathrm{H}} .
$$

$\operatorname{Assim}\left(\Pi_{1}\left(\mathcal{F}^{\mathbb{O H}}\right) / \mathbb{O}(n) \ltimes E\right)(x) \subset L_{x}^{\mathrm{H}}$.

- Seja $y \in L_{x}^{\mathrm{H}}$. Então, existe $\alpha \in C_{\text {par. }}^{\infty}\left([0,1], \mathcal{F}_{M}\right)$, tal que

$$
y=\mathrm{P}_{\alpha}(x)
$$


Tome $\xi \in \mathbb{O}\left(E_{\pi(x)}\right)$. Defina $\tilde{\alpha} \in C_{\text {par. }}^{\infty}\left([0,1], \mathcal{F}^{\mathbb{O H}}\right)$ por $\tilde{\alpha}(t):=\mathrm{P}_{\alpha}^{t} \circ \xi$, de modo que $\tilde{\alpha}(0)=\xi$ e $\tilde{\alpha}(1)=\mathrm{P}_{\alpha} \circ \xi$.

Logo

$$
y=\left(\left(\mathrm{P}_{\alpha} \circ \xi\right) \circ \xi^{-1}\right)(x)=\left(\tilde{\alpha}(1) \circ \tilde{\alpha}(0)^{-1}\right)(x) \in\left(\frac{\Pi_{1}\left(\mathcal{F}^{\mathbb{O H}}\right)}{\mathbb{O}(n)} \ltimes E\right)(x) .
$$

$\operatorname{Assim} L_{x}^{\mathrm{H}} \subset\left(\Pi_{1}\left(\mathcal{F}^{\mathbb{O H}}\right) / \mathbb{O}(n) \ltimes E\right)(x)$.

\subsection{Folheação linearizada (Teorema B)}

A presente seção será dedicada a mostrar que a linearização de uma FRS é dada por órbitas de um grupóide de Lie, o que será feito por meio do Teorema 4.19 na seção 4.3.2 (Teorema B apresentado no início deste capítulo). A prova deste teorema seguirá de perto a prova do Teorema 4.12 (apresentada na seção anterior), onde foi necessário o controle da dimensão dos grupos de holonomia da distribuição horizontal $\mathcal{T}^{\ell}$. Já na prova do Teorema 4.19 será necessário o controle da dimensão dos grupos de isometria que fixam a folheação infinitesimal (definidos na seção 2.2) e, com esta finalidade, será relembrado, rapidamente, a definição da distribuição $\mathcal{N}$ (apresentada na Seção 4.1) e será mostrada uma propriedade importante desta distribuição que, entre outras coisas, possibilitará o controle da dimensão dos grupos de isometria citados.

Sejam $(M, g)$ uma variedade Riemanniana, $\mathcal{F}$ uma FRS e $B \subset M$ subvariedade fechada, saturada e contida em um estrato, de modo que $\mathcal{F}_{B}$ é uma folheação (regular) de $B$.

Dada $W=\exp \left(\nu^{\varepsilon} B\right)$ uma $\varepsilon$-VTD, com projeção na base $B$ denotada por $\rho$, será denotado por $W_{b}$ a fibra de $W$ sobre $b \in B$. Será considerada $\mathcal{N} \subset \mathrm{T} W$ a distribuição (regular) dada, em cada ponto $p \in W$, pelo complemento $g_{\rho(p)}$-ortogonal de $\operatorname{ker}\left(\left.\mathrm{d} \rho\right|_{p}\right)$ em $\mathrm{T}_{p} S_{\rho(p)}^{\varepsilon}$ (ver Figura 4.3).

A cerca da distribuição $\mathcal{N}$, tem-se a seguinte proposição.

Proposição 4.13) ([7]) Sejam $P$ uma placa de $\mathcal{F}$ em $B$ e $X \in \mathfrak{X}_{P}(B) \cap \mathfrak{X}^{b}\left(\mathcal{F}_{B}\right)$ (i.e. um campo de $B$ ao longo de $P$ que é $\mathcal{F}_{B}$-básico). Então, existe $\widetilde{X} \in \mathfrak{X}_{\text {loc. }}(W)$, tal que

- $P \subset \operatorname{Dom}(\tilde{X}) \cap B$ e $\left.\tilde{X}\right|_{P}=X$,

- $\widetilde{X}$ é $\mathcal{F}$-folheado e é tangente a $\mathcal{N}$.

Dado $b \in B$ relembre, através da Proposição 2.18, que $\mathrm{K}_{b}^{0}$ era a componente conexa maximal, em torno da identidade, do subgrupo de isometrias Iso $\left(\mathcal{F}_{b}\right)$ que fixa a folheação infinitesimal $\mathcal{F}_{b}$, ou seja,

$$
\operatorname{Iso}\left(\mathcal{F}_{b}\right):=\left\{T \in \operatorname{Iso}\left(\nu_{b} B\right): T(L) \subset L, \forall L \in \mathcal{F}_{b}\right\}
$$


Dado $n \in \mathbb{Z}_{+}^{*}$ e $X \in \mathfrak{X}\left(\mathcal{F}_{W}\right)^{n}$ relembre, pela seção 2.2, que era possível considerar a sua linearização $X^{\ell} \in \mathfrak{X}\left(\mathcal{F}_{W}\right)^{n}$, em torno de $B$. Neste caso, considerando-se $\phi: D \subset \mathbb{R} \times W \longrightarrow W$ o fluxo desta linearização e $D_{t}:=\{p \in W:(t, p) \in D\}$, para cada $b \in \rho\left(\mathrm{D}_{t}\right)$ e $t \in \mathbb{R}$, tal que $D_{t} \neq \emptyset$, tinha-se que $D_{t} \cap W_{b}=W_{b}$ e a aplicação $\left.\phi_{t}\right|_{W_{b}}: W_{b} \longrightarrow W_{\phi_{t}(b)}$ era uma isometria (ver Proposição 2.15). Ainda, na seção citada, foi mostrado que era possível estender a isometria $\left.\phi_{t}\right|_{W_{b}}$, por meio de homotetias, a todo o espaço $\nu_{b} B$, para se obter uma isometria $\phi_{(t, b)}: \nu_{b} B \longrightarrow \nu_{\phi_{t}(b)} B$.

Corolário 4.14) ([5]) Considere $\widetilde{X} \in \mathfrak{X}_{\text {loc. }}(W)$ como descrito na proposição anterior e seja $\tilde{\phi}: \widetilde{D} \subset \mathbb{R} \times \operatorname{Dom}(\widetilde{X}) \longrightarrow \operatorname{Dom}(\widetilde{X})$ o fluxo de $\widetilde{X}^{\ell}$. Então, para cada $(t, b) \in \widetilde{D} \cap(\mathbb{R} \times B)$, a aplicação $\theta_{P, X}^{(t, b)}: \mathrm{K}_{b}^{0} \longrightarrow \mathrm{K}_{\tilde{\phi}_{t}(b)}^{0}$, definida por

$$
\theta_{P, X}^{(t, b)}\left(k_{b}\right):=\tilde{\phi}_{(t, b)} \circ k_{b} \circ\left(\tilde{\phi}_{(t, b)}\right)^{-1}
$$

é um isomorfismo de Lie, onde $\tilde{\phi}_{(t, b)}$ é a extensão homotética (ao espaço $\nu_{b} B$ ) de $\left.\tilde{\phi}_{t}\right|_{W_{b}}$.

Demonstração. A fim de simplificar a notação, fixados $t$ e $b$, a aplicação $\theta_{P, X}^{(t, b)}$ será simplesmente denotada por $\theta$ e o ponto $\tilde{\phi}_{t}(b)$ será denotado por $\tilde{b}$, ao longo da demonstração.

Será demonstrado, primeiramente, que $\theta$ está bem definida, ou seja, que $\theta\left(k_{b}\right) \in \mathrm{K}_{\tilde{b}}^{0}$, para todo $k_{b} \in \mathrm{K}_{b}^{0}$.

Seja $X \in \mathfrak{X}\left(\mathcal{F}_{b}\right)$, com $\phi: D^{X} \subset \mathbb{R} \times W_{b} \longrightarrow W_{b}$ o fluxo de $X^{\ell}$. Observe, pela Proposição 2.18, que $\phi_{s} \in \mathrm{K}_{b}^{0}$, para todo $s \in \mathbb{R}$, tal que $D_{s}^{X} \neq \emptyset$.

Defina $Y:=\left(\tilde{\phi}_{(t, b)}\right)_{*} X$, com $\psi: D^{Y} \subset \mathbb{R} \times W_{\tilde{b}} \longrightarrow W_{\tilde{b}}$ o fluxo de $Y^{\ell}$. Então

- $Y \in \mathfrak{X}\left(\mathcal{F}_{\tilde{b}}\right)$, pois $\tilde{\phi}_{t}$ é folheada, dado que $\widetilde{X}^{\ell}$ é $\mathcal{F}$-folheado, ou seja, $\tilde{\phi}_{(t, b)}$ é folheada.

- $\psi_{s}=\tilde{\phi}_{(t, b)} \circ \phi_{s} \circ\left(\tilde{\phi}_{(t, b)}\right)^{-1}$, pois $\tilde{\phi}_{(t, b)}$ é invariante por homotetias e, portanto, $Y^{\ell}=$ $\left(\tilde{\phi}_{(t, b)}\right)_{*} X^{\ell}$, ou seja, $Y^{\ell}$ está $\tilde{\phi}_{(t, b)}$-relacionado com $X^{\ell}$.

Assim, pela Proposição 2.18, tem-se que $\theta\left(\phi_{s}\right)=\psi_{s} \in K_{\tilde{b}}^{0}$, para todo $s \in \mathbb{R}$, tal que $D_{s}^{X} \neq \emptyset$.

Conclui-se que, dado $k_{b} \in \mathrm{K}_{b}^{0}$, tem-se que $\left.\tilde{\phi}_{(t, b)}\right|_{W_{b}} \circ k_{b} \circ\left(\tilde{\phi}_{(t, b)}\right)^{-1} \in \mathrm{K}_{\tilde{b}}^{0}$. De fato, pela Proposição 2.18, existem $\left(s_{1}, \cdots, s_{n}\right) \in \mathbb{R}^{n}$ e $X_{1}, \cdots, X_{n} \in \mathfrak{X}\left(\mathcal{F}_{b}\right)$, tal que $k_{b}=\phi_{s_{n}}^{n} \circ \cdots \circ \phi_{s_{1}}^{1}$, onde $\phi^{i}$ é o fluxo de $X_{i}^{\ell}$, para todo $i \in\{1, \cdots, n\}$ e, portanto, $\theta\left(k_{b}\right)=\theta\left(\phi_{s_{n}}^{n}\right) \circ \cdots \circ \theta\left(\phi_{s_{1}}^{1}\right) \in \mathrm{K}_{\tilde{b}}^{0}$.

A seguir, conclui-se que $\theta$ é um isomorfismo de Lie.

É trivial ver que $\theta$ é um isomorfismo (de grupos), uma vez que $\theta$ é uma conjugação pela aplicação $\left.\tilde{\phi}_{t}\right|_{W_{b}}$. Ainda, $\bar{\theta}: \mathbb{G} \mathbb{L}\left(E_{b}\right) \longrightarrow \mathbb{G L}\left(E_{\tilde{b}}\right)$ dada por

$$
\bar{\theta}\left(T_{b}\right):=\tilde{\phi}_{(t, b)} \circ T_{b} \circ\left(\tilde{\phi}_{(t, b)}\right)^{-1}
$$

é uma aplicação linear (pois é uma conjugação por uma aplicação linear) e, portanto, suave. Logo, como $\mathrm{K}_{\tilde{b}}^{0} \subset \mathbb{O}\left(E_{\tilde{b}}\right)$ é uma subvariedade fracamente mergulhada e $\mathbb{O}\left(E_{\tilde{b}}\right) \subset \mathbb{G} \mathbb{L}\left(E_{\tilde{b}}\right)$ é mergulhada, segue que $\mathrm{K}_{\tilde{b}}^{0} \subset \mathbb{G} \mathbb{L}\left(E_{\tilde{b}}\right)$ é fracamente mergulhada e, portanto, $\theta$ é suave. Argumentando-se de modo análogo, tem-se que $\theta^{-1}$ também é suave. 
Assim $\theta$ é um ismorfismo de Lie.

Corolário 4.15) A dimensão dos grupos $\mathrm{K}_{b}^{0}$ é constante ao longo de $B$.

Demonstração. Dado $L \in \mathcal{F}$, considere $\mathcal{C}_{L}$ uma cobertura de $L$ por placas. Uma vez que $\mathfrak{X}_{P}(B) \cap \mathfrak{X}^{b}\left(\mathcal{F}_{B}\right) \neq \emptyset$, para cada $P \in \mathcal{C}_{L}$ e $L \in \mathcal{F}$, tem-se que

$$
\mathcal{C}:=\left\{\operatorname{Dom}(\tilde{X}) \cap B: X \in \mathfrak{X}_{P}(W) \cap \mathfrak{X}^{b}\left(\mathcal{F}_{B}\right), P \in \mathcal{C}_{L} \text { e } L \in \mathcal{F}\right\}
$$

é uma cobertura aberta de $B$, onde $\tilde{X} \in \mathfrak{X}_{\text {loc. }}$ (W) é a extensão de $X$ obtida no corolário anterior.

Assim, pelo corolário anterior, $b \longmapsto \operatorname{dim}\left(\mathrm{K}_{b}^{0}\right)$ é constante em cada elemento da cobertura $\mathcal{C}$ e, portanto, é constatante em $B$.

\subsubsection{Construções preliminares à prova do Teorema B}

Será apresentado, na seção 4.3.2, o enunciado preciso e a prova do Teorema B (descrito no início deste capítulo).

Antes de apresentá-los, serão enunciados nesta seção alguns lemas e proposições necessários à prova de tal teorema. O leitor que se sentir confortável poderá, simplesmente, ignorar a prova dos mesmos e, apenas, ler e compreender o enunciado de tais lemas e proposições. Isto não trará prejuízos futuros à compreensão de como os mesmo serão utilizados na prova do Teorema $\mathrm{B}$, ou seja, nenhuma informação contida na prova de tais lemas e proposições, será diretamente utilizada na prova deste teorema.

A compreensão dos lemas e das proposições que precedem o Teorema $\mathrm{B}$, exige que se estabeleça alguma notação preliminar, como será feito a seguir.

Considere $\mathbb{O}(\nu B)$ o $\mathbb{O}(n)$-fibrado principal de referênciais ortogonais do fibrado $\nu B$, ou seja, $\mathbb{O}\left(\nu_{b} B\right)=\sqcup_{b \in B}$ Iso $\left(\mathbb{R}^{n}, \nu_{b} B\right)$, onde Iso $\left(\mathbb{R}^{n}, \nu_{b} B\right)$ é o conjunto da isometrias (lineares) de $\mathbb{R}^{n}$ em $\nu_{b} B$. Sem o risco de confusão e com um abuso de notação, será denotado por $\pi$ a projeção do fibrado $\mathbb{O}(\nu B)$ na base $B$.

Dado $X \in \mathfrak{X}\left(\mathcal{F}_{W}\right)$, como visto na seção 2.2 , é possível considerar $X^{\ell} \in \mathfrak{X}\left(\mathcal{F}_{W}\right)$ a linearização de $X$, cujo fluxo será denotado por $\phi^{X^{\ell}}: D^{X^{\ell}} \subset \mathbb{R} \times W \longrightarrow W$. Defina o aberto $\widetilde{D}^{X^{\ell}} \subset \mathbb{R} \times \mathbb{O}(\nu B)$ por

$$
\widetilde{D}^{X^{\ell}}:=\left\{(t, \eta) \in \mathbb{R} \times \mathbb{O}(\nu B): D_{t}^{X^{\ell}} \neq \emptyset \text { e } \pi(\eta) \in \pi\left(D_{t}^{X^{\ell}}\right)\right\}
$$

e o fluxo local $\Phi^{X^{\ell}}: \widetilde{D}^{X^{\ell}} \subset \mathbb{R} \times \mathbb{O}(\nu B) \longrightarrow \mathbb{O}(\nu B)$ por

$$
\Phi^{X^{\ell}}(t, \eta):=\phi_{t}^{X^{\ell}} \circ \eta
$$

Aplicando-se o Teorema de Sussmann (Teorema 1.12) ao subconjunto dos campos vetoriais gerado pelo conjunto de fluxos locais $\left\{\Phi^{X^{\ell}}: X \in \mathfrak{X}\left(\mathcal{F}_{W}\right)\right\}$, obtém-se uma folheação singular, 
que será denotada por $\mathbb{O} \mathcal{F}^{\ell}$ e cuja folha passando por $\eta \in \mathbb{O}(\nu B)$ é dada por

$$
\mathbb{O} L_{\eta}^{\ell}:=\left\{\phi_{t}^{X^{\ell}} \circ \eta \in \mathbb{O}(\nu B): X \in \mathfrak{X}\left(\mathcal{F}_{W}\right)^{n}, t \in I^{X^{\ell}} \subset \mathbb{R}^{n}, n \in \mathbb{Z}_{+}^{*}\right\} .
$$

Vale observar que, com esta folheação em $\mathbb{O}(\nu B)$, a projeção $\pi: \mathbb{O}(\nu B) \longrightarrow B$ é folheada, dado que os campos linearizados são projetáveis, ou seja, $\pi$-invariantes (ver Proposição 2.14) e, para cada $u \in \mathbb{R}^{n}$, a aplicação $\pi_{u}: \mathbb{O}(\nu B) \longrightarrow \nu B$ dada por $\pi_{u}(\eta):=\eta(u)$ é uma submersão folheada, considerando-se $\nu B$ folheada pela extensão homotética da folheação linearizada $\mathcal{F}^{\ell}$, cuja folha passando por $p \in W$ é dada por

$$
L_{p}^{\ell}=\left\{\phi_{t}^{X^{\ell}}(p) \in W: X \in \mathfrak{X}\left(\mathcal{F}_{W}\right)^{n}, t \in I^{X^{\ell}} \subset \mathbb{R}^{n}, n \in \mathbb{Z}_{+}^{*}: p \in D_{t}^{X^{\ell}}\right\}
$$

(ver seção 2.2, para maiores detalhes a cerca da extensão homotética da folheação linearizada). Em vista desta observação, a folheação $\mathbb{O} \mathcal{F}^{\ell}$ será dita o levantamento ortogonal da folheação $\underline{\mathcal{F}^{\ell}}$.

Analogamente, é possível considerar $\mathbb{O}^{\text {lc }}$ o levantamento ortogonal de $\mathcal{F}^{\text {lc }}$.

A seguir será provado na Proposição 4.18 que a folheação $\mathbb{O} \mathcal{F}^{\ell}$ é regular. Isto será feito por meio do Lema 4.16 e do Lema 4.17, que mostra que a escolha de uma extensão $\mathcal{T} \subset \mathrm{T} \mathcal{F}_{W}$ de $\mathrm{T} \mathcal{F}_{B}$ decompõe o plano tangente as $\mathbb{O} \mathcal{F}^{\ell}$-folhas em duas direções, a saber, a direção da distribuição horizontal $\mathbb{O} \mathcal{T}^{\ell}$ induzida por $\mathcal{T}^{\ell}$ e a direção tangente as órbitas dos grupos $\mathrm{K}_{b}^{0}$.

\section{Lema 4.16)}

a) A ação de $\mathrm{K}_{b}^{0}$ sobre $\mathbb{O}\left(\nu_{b} B\right)$ dada por $k_{b} \cdot \eta:=k_{b} \circ \eta$ é livre, para todo $b \in B$. Em particular, $\operatorname{dim}\left(\mathrm{K}_{b}^{0}\right)=\operatorname{dim}\left(\mathrm{K}_{b}^{0}(\eta)\right)$, para todo $\eta \in \mathbb{O}(\nu B)$, com $\pi(\eta)=b$.

b) A componente conexa de $\mathbb{O}\left(\nu_{b} B\right) \cap \mathbb{O} L_{\eta}^{\ell}$ é igual a $\mathrm{K}_{b}^{0}(\eta)$, para todo $\eta \in \mathbb{O}(\nu B)$, com $\pi(\eta)=b$.

\section{Demonstração.}

a) Uma vez que uma isometria de $\nu_{b} B$ que fixa todos os pontos é, necessariamente, a identidade, segue que

$$
\bigcap_{x \in \nu_{b} B}\left(\mathrm{~K}_{b}^{0}\right)_{x} \subset \bigcap_{x \in \nu_{b} B} \operatorname{Iso}\left(\nu_{b} B\right)_{x}=\{\operatorname{Id}\}
$$

e, portanto, a ação de $\mathrm{K}_{b}^{0}$ sobre $\nu_{b} B$ é efetiva.

Logo, a ação de $\mathrm{K}_{b}^{0}$ sobre $\mathbb{O}\left(\nu_{b} B\right)$ é livre. De fato, dado $\eta \in \mathbb{O}\left(\nu_{b} B\right)$ e $k_{b} \in\left(\mathrm{K}_{b}^{0}\right)_{\eta}$, tem-se que $k_{b} \circ \eta=\eta$ e, portanto,

$$
k_{b} \in \bigcap_{u \in \mathbb{R}^{n}}\left(\mathrm{~K}_{b}^{0}\right)_{\eta(u)}=\bigcap_{x \in \nu_{b} B}\left(\mathrm{~K}_{b}^{0}\right)_{x}=\{\operatorname{Id}\} .
$$

$\operatorname{Assim} \mathrm{K}_{b}^{0}=\mathrm{K}_{b}^{0} /\left(\mathrm{K}_{b}^{0}\right)_{\eta} \approx \mathrm{K}_{b}^{0}(\eta)$ e, portanto, $\operatorname{dim}\left(\mathrm{K}_{b}^{0}\right)=\operatorname{dim}\left(\mathrm{K}_{b}^{0}(\eta)\right)$ 
b) Seja $\tilde{\eta} \in \mathbb{O}\left(\nu_{b} B\right) \cap \mathbb{O} L_{\eta}^{\ell}$. Então existem $n \in \mathbb{Z}_{+}^{*}, X \in \mathfrak{X}\left(\mathcal{F}_{W}\right)^{n}$ e $t \in \mathbb{R}^{n}$, tais que $\tilde{\eta}=\phi_{t}^{X^{\ell}} \circ \eta$

Uma vez que $\pi(\eta)=b=\pi(\tilde{\eta})$ e o fluxo de campos linearizados tangentes à folheação são isometrias (ver Proposição 2.15), segue que $\phi_{t}^{X^{\ell}} \in \operatorname{Iso}\left(\nu_{b} B\right)$. Ainda, como $X^{\ell} \in \mathfrak{X}\left(\mathcal{F}_{W}\right)^{n}$, tem-se que $\phi_{t}^{X^{\ell}}$ fixa a folheação $\mathcal{F}$ e, portanto, fixa a folheação infinitesimal $\mathcal{F}_{b}$, ou seja, $\phi_{t}^{X^{\ell}} \in \operatorname{Iso}\left(\mathcal{F}_{b}\right)$.

$\operatorname{Assim} \mathbb{O}\left(\nu_{b} B\right) \cap \mathbb{O} L_{\eta}^{\ell} \subset \operatorname{Iso}\left(\mathcal{F}_{b}\right)(\eta)$ e, portanto, a componente conexa de $\mathbb{O}\left(\nu_{b} B\right) \cap \mathbb{O} L_{\eta}^{\ell}$ está contida em $\mathrm{K}_{b}^{0}(\eta)$.

Seja $\tilde{\eta} \in \mathrm{K}_{b}^{0}(\eta)$. Então existem $k_{b} \in \mathrm{K}_{b}^{0}$, tal que $\tilde{\eta}=k_{b} \circ \eta$ e, pelo Lema 2.21, existem $n \in \mathbb{Z}_{+}^{*}, X \in \mathfrak{X}\left(\mathcal{F}_{W}\right)^{n}$ e $t \in \mathbb{R}^{n}$, tal que $k_{b}=\left.\phi_{t}^{X^{\ell}}\right|_{\nu_{b} B}$.

Logo $\tilde{\eta}=\phi_{t}^{X^{\ell}} \circ \eta \in \mathbb{O}\left(\nu_{b} B\right) \cap \mathbb{O} L_{\eta}^{\ell}$.

Assim $\mathbb{O}\left(\nu_{b} B\right) \cap \mathbb{O} L_{\eta}^{\ell} \supset \mathrm{K}_{b}^{0}(\eta)$ e, como $\mathrm{K}_{b}^{0}$ é a componente conexa de $\operatorname{Iso}\left(\mathcal{F}_{b}\right)$, segue que a componente conexa de $\mathbb{O}\left(\nu_{b} B\right) \cap \mathbb{O} L_{\eta}^{\ell}$ contém $\mathrm{K}_{b}^{0}(\eta)$.

Dada $\mathcal{T} \subset \mathrm{T} \mathcal{F}_{W}$ uma extensão de $\mathrm{TF}_{B}$, como construída na seção 4.1, foi visto que a linearização de $\mathcal{T}$ induzia uma distribuição horizontal, em $\nu B$, ao longo de $\mathrm{T} \mathcal{F}_{B}$, tal que a conexão associada a $\mathcal{T}^{\ell}$ era compatível com a métrica $\left.g\right|_{\nu B}$, de modo que, para cada $\alpha \in C_{\text {par. }}^{\infty}\left([0,1], \mathcal{F}_{B}\right)$, o operador de transporte paralelo $\mathrm{P}_{\alpha}^{t}: \nu_{\alpha(0)} B \longrightarrow \nu_{\alpha(t)} B$ era uma isometria (linear). Considere, neste caso, $\mathbb{O} \mathcal{T}^{\ell} \subset \mathrm{T} \mathbb{O}(\nu B)$ a distribuição horizontal, em $\mathbb{O}(\nu B)$, ao longo de $\mathrm{T} \mathcal{F}_{B}$, induzida por $\mathcal{T}^{\ell} \subset \mathrm{T}(\nu B)$ (ver [33], por exemplo, para a construção de $\mathbb{O} \mathcal{T}^{\ell}$ ). A saber, para cada $\eta \in \mathbb{O}(\nu B)$,

$$
\left.\mathbb{O} \mathcal{T}^{\ell}\right|_{\eta}=\operatorname{span}\left(\left\{\left.\frac{\mathrm{d}}{\mathrm{d} t} \mathrm{P}_{\alpha}^{t} \circ \eta\right|_{t=0} \in \mathrm{T}_{\eta} \mathbb{O}(\nu B): \alpha \in C_{\text {par. }}^{\infty}\left([0,1] ; \mathcal{F}_{B}\right)\right\}\right) .
$$

Lema 4.17) Seja $\mathcal{T} \subset \mathrm{T} \mathcal{F}_{W}$ uma extensão de $\mathrm{T} \mathcal{F}_{B}$, tal que $\mathcal{T} \cap \operatorname{Ker}(\mathrm{d} \rho)=\{0\}$. Então

a) $\mathbb{O} \mathcal{T}^{\ell} \subset \mathrm{T}\left(\mathbb{O} \mathcal{F}^{\ell}\right)$

b) $\mathrm{T}_{\eta} \mathbb{O} L_{\eta}^{\ell}=\left.\mathbb{O} \mathcal{T}^{\ell}\right|_{\eta} \oplus \mathrm{T}_{\eta} \mathrm{K}_{b}^{0}(\eta)$, para todo $\eta \in \mathbb{O}(\nu B)$, com $\pi(\eta)=b$.

Demonstração. Fixe $\eta \in \mathbb{O}(\nu B)$, com $\pi(\eta)=b$.

a) Seja $\left.\left.\frac{\mathrm{d}}{\mathrm{d} t} \mathrm{P}_{\alpha} \circ \eta\right|_{t=0} \in \mathbb{O} \mathcal{T}^{\ell}\right|_{\eta}$.

Uma vez que $\alpha \in C_{\text {par. }}^{\infty}\left([0,1], \mathcal{F}_{B}\right)$, tem-se que $\alpha=\alpha_{n} * \cdots * \alpha_{1}$, com $\alpha_{i} \in C^{\infty}\left([0,1], \mathcal{F}_{B}\right)$ regular, para cada $i \in\{1, \cdots, n\}$ e, consequentemente, $\left.\frac{\mathrm{d}}{\mathrm{d} t} \mathrm{P}_{\alpha}^{t} \circ \eta\right|_{t=0}=\left.\frac{\mathrm{d}}{\mathrm{d} t} \mathrm{P}_{\alpha_{1}}^{t} \circ \eta\right|_{t=0}$.

Existe $X_{1} \in \mathfrak{X}(B)$, tal que $\alpha_{1}$ é um segmento de curva integral de $X_{1}$. Considere $\bar{X}_{1}$ o levantamento $\mathcal{T}^{\ell}$-horizontal de $X_{1}$, de modo que $\mathrm{P}_{\alpha_{1}}^{t}=\left.\phi_{t}^{\bar{X}_{1}}\right|_{\nu_{b} B}$, para todo $t \in I^{\bar{X}_{1}} \subset \mathbb{R}$, onde $\phi^{\bar{X}_{1}}: D^{\bar{X}_{1}} \subset \mathbb{R} \times \nu B \longrightarrow \nu B$ é o fluxo de $\bar{X}_{1}$.

Logo $\mathrm{P}_{\alpha_{1}}^{t} \circ \eta=\phi_{t}^{\bar{X}_{1}} \circ \eta$ e, portanto, a curva $t \longmapsto \mathrm{P}_{\alpha_{1}}^{t} \circ \eta$ está contida na folha $\mathbb{O} L_{\eta}^{\ell}$. $\left.\operatorname{Assim} \frac{\mathrm{d}}{\mathrm{d} t} \mathrm{P}_{\alpha} \circ \eta\right|_{t=0} \in \mathrm{T}_{\eta} \mathbb{O} L_{\eta}^{\ell}$.

b) Observe que, para cada $\eta \in \mathbb{O}(\nu B) \operatorname{com} \pi(\eta)=b$, tem-se que 
- $\mathbb{O}\left(\nu_{b} B\right) \cap \mathbb{O} L_{\eta}^{\ell}=\mathrm{K}_{b}^{0}(\eta)$, pelo item (a) do Lema (b),

- $\left.\mathbb{O} \mathcal{T}^{\ell}\right|_{\eta} \cap \mathrm{T}_{\eta} \mathbb{O}\left(\nu_{b} B\right)=\{0\}$, dado que $\mathbb{O} \mathcal{T}^{\ell}$ é uma distribuição horizontal em $\mathbb{O}(E)$,

de modo que resta apenas demonstrar que $\mathrm{T}_{\eta} \mathbb{O} L_{\eta}^{\ell}=\left.\mathbb{O} \mathcal{T}^{\ell}\right|_{\eta}+\mathrm{T}_{\eta}\left(\mathbb{O}\left(\nu_{b} B\right) \cap \mathbb{O} L_{\eta}^{\ell}\right)$.

Seja $v \in \mathrm{T}_{\eta} \mathbb{O} L_{\eta}^{\ell}$. Tome $\tilde{\alpha} \in C^{\infty}\left([0,1], \mathbb{O} L_{\eta}^{\ell}\right)$, tal que $\tilde{\alpha}(0)=\eta$ e $\tilde{\alpha}^{\prime}(0)=v$ e defina $\alpha \in C^{\infty}([0,1], B)$ por $\alpha:=\pi \circ \tilde{\alpha}$. Dado que $\pi$ é folheada e $\operatorname{Im}(\tilde{\alpha}) \subset \mathbb{O} L_{\eta}^{\ell}$, tem-se que $\alpha \in C^{\infty}\left([0,1], \mathcal{F}_{B}\right)$.

Segue que

$$
\left.\mathrm{d} \pi\right|_{\eta}\left(\tilde{\alpha}^{\prime}(0)-\left.\frac{\mathrm{d}}{\mathrm{d} t} \mathrm{P}_{\alpha}^{t} \circ \eta\right|_{t=0}\right)=\left.\frac{\mathrm{d}}{\mathrm{d} t} \pi(\alpha(t))\right|_{t=0}-\left.\frac{\mathrm{d}}{\mathrm{d} t} \pi\left(\mathrm{P}_{\alpha}^{t} \circ \eta\right)\right|_{t=0}=0
$$

ou seja,

$$
\tilde{\alpha}^{\prime}(0)-\left.\frac{\mathrm{d}}{\mathrm{d} t} \mathrm{P}_{\alpha}^{t} \circ \eta\right|_{t=0} \in \operatorname{Ker}\left(\left.\mathrm{d} \pi\right|_{\eta}\right)=\mathrm{T}_{\eta} \mathbb{O}\left(\nu_{b} B\right) .
$$

Logo, como $\left.\frac{\mathrm{d}}{\mathrm{d} t} \mathrm{P}_{\alpha}^{t} \circ \eta\right|_{t=0} \in \mathbb{O} \mathcal{T}^{\ell} \subset \mathrm{T}_{\eta} \mathbb{O} L_{\eta}^{\ell}$, conclui-se que

$$
\tilde{\alpha}^{\prime}(0)-\left.\frac{\mathrm{d}}{\mathrm{d} t} \mathrm{P}_{\alpha}^{t} \circ \eta\right|_{t=0} \in \mathrm{T}_{\eta}\left(\mathbb{O}\left(\nu_{b} B\right) \cap \mathbb{O} L_{\eta}^{\ell}\right) .
$$

Assim, dado que

$$
v=\left.\frac{\mathrm{d}}{\mathrm{d} t} \mathrm{P}_{\alpha}^{t} \circ \eta\right|_{t=0}+\left(\tilde{\alpha}^{\prime}(0)-\left.\frac{\mathrm{d}}{\mathrm{d} t} \mathrm{P}_{\alpha}^{t} \circ \eta\right|_{t=0}\right),
$$

segue que $\left.v \in \mathbb{O} \mathcal{T}^{\ell}\right|_{\eta}+\mathrm{T}_{\eta}\left(\mathbb{O}\left(\nu_{b} B\right) \cap \mathbb{O} L_{\eta}^{\ell}\right)$.

Proposição 4.18) $\mathbb{O} \mathcal{F}^{\ell}$ é regular.

Demonstração. Considere $\mathcal{T} \subset \mathrm{T} \mathcal{F}_{W}$ uma extensão de $\mathrm{TF}_{B}$, tal que $\mathcal{T} \cap \operatorname{Ker}(\mathrm{d} \rho)=\{0\}$. Segue, dos Lemas 4.16 e 4.17, que

$$
\operatorname{dim}\left(\mathbb{O} L_{\eta}^{\ell}\right)=\operatorname{dim}\left(\left.\mathbb{O} \mathcal{T}^{\ell}\right|_{\eta}\right)+\operatorname{dim}\left(\mathbb{O}\left(\nu_{b} B\right) \cap \mathbb{O} L_{\eta}^{\ell}\right)=\operatorname{posto}\left(\mathbb{O} \mathcal{T}^{\ell}\right)+\operatorname{dim}\left(\mathrm{K}_{\pi(\eta)}^{0}\right)
$$

para todo $\eta \in \mathbb{O}(\nu B)$. Mas, pelo Corolário 4.15, tem-se que $\eta \longmapsto \operatorname{dim}\left(\mathrm{K}_{\pi(\eta)}^{0}\right)$ é constante.

$\operatorname{Assim} \eta \longmapsto \operatorname{dim}\left(\mathbb{O} L_{\eta}^{\ell}\right)$ é constante e, portanto, $\mathbb{O} \mathcal{F}^{\ell}$ é regular.

Vale ressaltar que a regularidade de $\mathbb{O} \mathcal{F}^{\ell}$ independeu da escolha da extensão $\mathcal{T}$, que apenas determinou uma decomposição do plano tangente as folhas de $\mathbb{O} \mathcal{F}^{\ell}$, dentre muitas possíveis.

Sabendo-se que $\mathbb{O} \mathcal{F}^{\ell}$ é regular, devido a proposição anterior, é possível considerar $\operatorname{Mon}\left(\mathbb{O} \mathcal{F}^{\ell}\right):=\Pi_{1}\left(\mathbb{O} \mathcal{F}^{\ell}\right) \rightrightarrows \mathbb{O}(\nu B)$ o grupóide de monodromia da folheação $\mathbb{O} \mathcal{F}^{\ell}$, cujo o conjunto 
das flechas $\Pi_{1}\left(\mathbb{O} \mathcal{F}^{\ell}\right)$ é dado pelas classes de curvas $\mathbb{O} \mathcal{F}^{\ell}$-homotópicas (ver seção 3.2 para definição precisa deste grupóide).

Considere $\mu_{0}: \mathbb{O}(\nu B) \times \mathbb{O}(n) \longrightarrow \mathbb{O}(\nu B)$ a ação (à direita) canônica de $\mathbb{O}(n)$ sobre $\mathbb{O}(\nu B)$, a saber, dada por $\mu_{0}(\eta, T)=\eta \circ T$ e, para cada $T \in \mathbb{O}(n)$, considere $\mu_{0}^{T}: \mathbb{O}(\nu B) \longrightarrow \mathbb{O}(\nu B)$ o isomorfismo de fibrados dado por $\mu_{0}^{T}(\eta)=\mu_{0}(\eta, T)=\eta \circ T$.

Observe que $\mu_{0}^{T}$ é $\mathbb{O} \mathcal{F}^{\ell}$-folheado, pois $\mu_{0}^{T}\left(\phi_{t}^{X^{\ell}} \circ \eta\right)=\phi_{t}^{X^{\ell}} \circ \mu_{0}^{T}(\eta)$, para todo $X \in \mathfrak{X}\left(\mathcal{F}_{W}\right)$ e $t \in I^{X^{\ell}} \subset \mathbb{R}$.

A fim de não sobrecarregar a notação, quando não houver necessidade de explicitar a ação $\mu_{0}$, será denotado $\eta \cdot T:=\mu_{0}^{T}(\eta)$ e, para cada $\alpha \in C^{\infty}([0,1], \mathbb{O}(\nu B))$, será denotado $\alpha \cdot T:=\mu_{0}^{T} \circ \alpha$.

Defina $\mu_{1}: \Pi_{1}\left(\mathcal{F}^{\mathbb{O H}}\right) \times \mathbb{O}(n) \longrightarrow \Pi_{1}\left(\mathcal{F}^{\mathbb{O H}}\right)$ por

$$
\mu_{1}([\tilde{\alpha}], T):=[\tilde{\alpha} \cdot T]
$$

Lema) $\mu_{1}$ é uma ação (à direita) suave.

Demonstração. Análoga a demonstração do Lema 4.9.

Proposição) O par de ações $\left(\mu_{0}, \mu_{1}\right)$ é uma ação (à direita) suave, livre e própria sobre $\operatorname{Mon}\left(\mathbb{O} \mathcal{F}^{\ell}\right)$ e $\operatorname{Mon}\left(\mathbb{O} \mathcal{F}^{\ell}\right) / \mathbb{O}(n)$ é um grupóide de Lie.

Demonstração. Análoga a demonstração da Proporsição 4.10.

Defina $\mu: \Pi_{1}\left(\mathbb{O} \mathcal{F}^{\ell}\right) / \mathbb{O}(n)_{s} \times{ }_{\pi} \nu B \longrightarrow \nu B$ por

$$
\mu([\alpha] \cdot \mathbb{O}(n), x)=\left(\alpha(1) \circ \alpha(0)^{-1}\right)(x) .
$$

onde $\alpha(0)^{-1}: \nu_{\alpha(0)} B \longrightarrow \mathbb{R}^{n}$ é a inversa da isometria $\alpha(0)$.

Intuitivamente, a aplicação $\mu$ consiste em tomar as coordenadas de $x$ na base $\alpha(0)$ e construir o vetor dado por essas mesmas coordenadas na base $\alpha(1)$.

Proposição) $\mu$ gera uma representação de $\Pi_{1}\left(\mathbb{O} \mathcal{F}^{\ell}\right) / \mathbb{O}(n)$ em $\nu B$.

Demonstração. Análoga a demonstração da Proposição 4.11

A seção será encerrada com a observação de que, sendo $\mu$ uma representação, é possível considerar o grupóide de transformação $\left(\Pi_{1}\left(\mathbb{O} \mathcal{F}^{\ell}\right) / \mathbb{O}(n)\right) \ltimes \nu B$ da representação $\mu$ (ver seção 3.3.1 para a definição precisa deste grupóide) e este é, precisamente, o grupóide cujas órbitas são as folhas da folheação linearizada $\mathcal{F}^{\ell}$, como será visto na seção a seguir. 


\subsubsection{Teorema B}

Será apresentado, nesta breve seção, o enunciado preciso e a prova do Teorema B descrito na introdução e no início deste capítulo.

Teorema 4.19) Sejam $(M, g)$ uma variedade Riemanniana, $\mathcal{F}$ uma FRS e $B \subset M$ uma subvariedade fechada, saturada e contida em um extrato.

Então, existe $W$ uma $\epsilon$-vizinhança tubular distinta em torno de $B$, tal que $\mathcal{F}^{\ell}$ (resp. $\mathcal{F}^{\text {lc }}$ ) é isomorfa a folheação dada pelas órbitas de $\left(\Pi_{1}\left(\mathbb{O} \mathcal{F}^{\ell}\right) / \mathbb{O}(n)\right) \ltimes \nu B\left(\operatorname{resp} .\left(\Pi_{1}\left(\mathbb{O} \mathcal{F}^{\text {lc }}\right) / \mathbb{O}(n)\right) \ltimes \nu B\right)$, em $\nu^{\varepsilon} B$.

\section{Demonstração.}

- Seja $y \in\left(\Pi_{1}\left(\mathbb{O} \mathcal{F}^{\ell}\right) / \mathbb{O}(n) \ltimes \nu B\right)(x)$. Então, existe $\alpha \in C^{\infty}\left([0,1], \mathbb{O} \mathcal{F}^{\ell}\right)$, tal que

$$
y=\left(\alpha(1) \circ \alpha(0)^{-1}\right)(x) .
$$

Mas $\alpha(1) \in \mathbb{O} L_{\alpha(0)}^{\ell}$ e, portanto, existem $n \in \mathbb{Z}_{+}^{*}, X \in \mathfrak{X}\left(\mathcal{F}_{W}\right)^{n}$ e $t \in \mathbb{R}^{n}$, tal que $\alpha(1)=\phi_{t}^{X^{\ell}} \circ \alpha(0)$.

Logo

$$
y=\left(\phi_{t}^{X^{\ell}} \circ \alpha(0) \circ \alpha(0)^{-1}\right)(x)=\phi_{t}^{X^{\ell}}(x) \in L_{x}^{\ell} .
$$

$\operatorname{Assim}\left(\Pi_{1}\left(\mathbb{O} \mathcal{F}^{\ell}\right) / \mathbb{O}(n) \ltimes \nu B\right)(x) \subset L_{x}^{\ell}$.

- Seja $y \in L_{x}^{\ell}$. Então, existem $n \in \mathbb{Z}_{+}^{*}, X \in \mathfrak{X}\left(\mathcal{F}_{W}\right)^{n}$ e $t \in \mathbb{R}^{n}$, tal que

$$
y=\phi_{t}^{X^{\ell}}(x)
$$

Tome $\eta \in \mathbb{O}\left(\nu_{\pi(x)} B\right)$ e, para cada $i \in\{1, \cdots, n\}$, defina $\alpha_{i} \in C^{\infty}\left([0,1], \mathbb{O} \mathcal{F}^{\ell}\right)$, por $\alpha_{i}(s):=\phi_{s t_{i}}^{X_{i}^{\ell}} \cdots \cdots \circ \phi_{t_{1}}^{X_{1}^{\ell}} \circ \eta$ e $\alpha \in C_{\text {par. }}^{\infty}\left([0,1], \mathbb{O} \mathcal{F}^{\ell}\right)$ por $\alpha:=\alpha_{n} * \cdots * \alpha_{1}$.

Segue que $\alpha(1)=\alpha_{n}(1)=\phi_{t}^{X^{\ell}} \circ \eta$ e $\alpha(0)=\alpha_{1}(0)=\eta$, ou seja,

$$
y=\left(\left(\phi_{t}^{X^{\ell}} \circ \eta\right) \circ \eta^{-1}\right)(x)=\left(\alpha(1) \circ \alpha(0)^{-1}\right)(x) \in\left(\frac{\Pi_{1}\left(\mathbb{O} \mathcal{F}^{\ell}\right)}{\mathbb{O}(n)} \ltimes \nu B\right)(x) .
$$

$\operatorname{Assim} L_{x}^{\ell} \subset\left(\Pi_{1}\left(\mathbb{O} \mathcal{F}^{\ell}\right) / \mathbb{O}(n) \ltimes \nu B\right)(x)$. 


\subsection{Um modelo semi-local (Teorema C)}

\subsubsection{Folheação de holonomia generalizada (modelo semi-local)}

Sejam $M$ uma variedade diferenciável, D $\subset \mathrm{T} M$ um subfibrado, $E$ um fibrado vetorial sobre $M$, com projeção $\pi$, e $\mathrm{H}$ uma distribuição horizontal ao longo de $D$.

Considere $C_{\text {par. }}^{\infty}([0,1], \mathrm{D})$ o conjunto das curvas suaves por partes tangentes a $D$ e $\mathrm{Hol} \rightrightarrows M$ o grupóide de holonomia de $\mathrm{H}$, a saber, $\mathrm{Hol}=\left\{\mathrm{P}_{\alpha}: \alpha \in C_{\text {par. }}^{\infty}([0,1], \mathrm{D})\right\}$, onde $\mathrm{P}_{\alpha}: E_{\alpha(0)} \longrightarrow E_{\alpha(1)}$ é o operador de transporte paralelo, para cada $\left.\alpha \in C_{\text {par. }}^{\infty}([0,1], \mathrm{D})\right\}$.

Dado $X \in \mathfrak{X}(\mathrm{D})$, considere $\widetilde{X}$ o levantamento H-horizontal de $X$, a saber, o único campo tangente a $\mathrm{H}$, tal que $\mathrm{d} \pi(\widetilde{X})=X \circ \pi$. Cabe ressaltar que denotando-se por $\phi^{\widetilde{X}}: D^{\widetilde{X}} \subset \mathbb{R} \times E \longrightarrow$ $E$ o fluxo de $\widetilde{X}$, tem-se que

$$
\left.\phi_{t}^{\widetilde{X}}\right|_{E_{p}}=\mathrm{P}_{\alpha_{p}}^{t}
$$

para todo $p \in \pi\left(D_{t}^{\widetilde{X}}\right)$ e $t \in \mathbb{R}$, tal que $D_{t}^{\widetilde{X}} \neq \emptyset$, onde $\alpha_{p}$ é a curva integral de $X$ passando por $p$.

$$
\mathfrak{X}^{\mathrm{H}}(E):=\left\{\widetilde{X} \in \mathfrak{X}(E): X \in \mathfrak{X}\left(\mathcal{F}_{B}\right)\right\}
$$

Seja $\mathcal{F}^{E}$ uma folheação singular em $E$, tal que $E_{p}$ é saturada e $\mathcal{F}_{p}:=\left.\mathcal{F}^{E}\right|_{E_{p}}$ é uma folheação infinitesimal, para todo $p \in M$, e $\mathrm{P}_{\alpha}$ é folheada, para todo $\alpha \in C_{\text {par. }}^{\infty}([0,1], \mathrm{D})$ (Hol-invariância da folheação $\mathcal{F}^{E}$ ).

Ao longo desta seção, a folha de $\mathcal{F}^{E}$ passando por $x \in E$ será denotada por $L_{x}^{E}$.

Aplicando-se o Teorema de Sussmann (Teorema 1.12) ao subconjunto dos campos vetoriais $\mathfrak{X}\left(H, \mathcal{F}^{E}\right):=\mathfrak{X}^{\mathrm{H}}(E) \cup \mathfrak{X}\left(\mathcal{F}^{E}\right) \subset \mathfrak{X}(E)$, obtém-se uma folheação singular, que será denotada por $\mathcal{F}\left(\mathrm{H}, \mathcal{F}^{E}\right)$ e cuja folha passando por $x \in E$ é dada por

$$
L_{x}=\left\{\phi_{t}^{\widetilde{X}}(x) \in E: \widetilde{X} \in \mathfrak{X}\left(\mathrm{H}, \mathcal{F}^{E}\right)^{n}, t \in I^{\widetilde{X}}: x \in D_{t}^{\widetilde{X}}, n \in \mathbb{Z}_{+}^{*}\right\} .
$$

A folheação $\mathcal{F}\left(H, \mathcal{F}^{E}\right)$ tem uma caracterização muito mais intuitiva, como será mostrado no item (b) do lema a seguir.

Lema 4.20) Seja $x \in E$. Então

a) $\operatorname{Hol}\left(L_{y}^{E}\right) \subset \operatorname{Hol}\left(L_{x}^{E}\right)$, para todo $y \in \operatorname{Hol}\left(L_{x}^{E}\right)$,

b) $L_{x}=\operatorname{Hol}\left(L_{x}^{E}\right)$.

\section{Demonstração.}

a) Considere $y=\mathrm{P}_{\alpha}(\tilde{x})$, com $\tilde{x} \in L_{x}^{E} \mathrm{e} \alpha \in C_{\text {par. }}^{\infty}([0,1], D)$.

Seja $\mathrm{P}_{\beta}(\tilde{y}) \in \operatorname{Hol}\left(L_{y}^{E}\right)$, com $\tilde{y} \in L_{y}^{E}$ e $\beta \in C_{\text {par. }}^{\infty}([0,1], D)$.

Então $\mathrm{P}_{\alpha^{-1}}(\tilde{y}) \in L_{\tilde{x}}^{E}$, devido a Hol-invariância de $\mathcal{F}^{E}$ e, como $L_{\tilde{x}}^{E}=L_{x}^{E}$, segue que $\mathrm{P}_{\alpha^{-1}}(\tilde{y}) \in L_{x}^{E}$. 
$\operatorname{Logo} \mathrm{P}_{\beta}(\tilde{y})=\mathrm{P}_{\beta * \alpha}\left(\mathrm{P}_{\alpha^{-}}(\tilde{y})\right) \in \operatorname{Hol}\left(L_{x}^{E}\right)$.

$\operatorname{Assim} \operatorname{Hol}\left(L_{y}^{E}\right) \subset \operatorname{Hol}\left(L_{x}^{E}\right)$.

b) Seja $y \in L_{x}$. Existem $m \in \mathbb{Z}_{+}^{*}, \beta \in C_{\text {par. }}^{\infty}([0,1], D)^{m}$ e $Y \in \mathfrak{X}\left(\mathcal{F}^{E}\right)^{m}$, tais que

$$
y=\left(\mathrm{P}_{\beta_{m}} \circ \phi_{s_{m}}^{Y_{m}} \circ \cdots \circ \mathrm{P}_{\beta_{1}} \circ \phi_{s_{1}}^{Y_{1}}\right)(x) .
$$

$\operatorname{Mas}\left(\mathrm{P}_{\beta_{m}} \circ \phi_{s_{m}}^{Y_{m}} \circ \cdots \circ \mathrm{P}_{\beta_{1}} \circ \phi_{s_{1}}^{Y_{1}}\right)(x) \in \operatorname{Hol}\left(L_{x}^{E}\right)$. De fato, por indução,

- se $m=1$, então $\left(\mathrm{P}_{\beta_{1}} \circ \phi_{s_{1}}^{Y_{1}}\right)(x) \in \operatorname{Hol}\left(L_{x}^{E}\right)$, pois $Y_{1} \in \mathfrak{X}\left(\mathcal{F}^{E}\right)$ e, portanto, $\phi_{s_{1}}^{Y_{1}}(x) \in L_{x}^{E}$,

- se a afirmação é válida para $k \in \mathbb{Z}_{+}^{*}$, então $y=\left(\mathrm{P}_{\beta_{k}} \circ \phi_{s_{k}}^{Y_{k}} \circ \cdots \circ \mathrm{P}_{\beta_{1}} \circ \phi_{s_{1}}^{Y_{1}}\right)(x) \in$ $\operatorname{Hol}\left(L_{x}^{E}\right)$ e, pelo item anterior, segue que

$$
\left(\mathrm{P}_{\beta_{(k+1)}} \circ \phi_{s_{(k+1)}}^{Y_{(k+1)}}\right)\left(\left(\mathrm{P}_{\beta_{k}} \circ \phi_{s_{k}}^{Y_{k}} \circ \cdots \circ \mathrm{P}_{\beta_{1}} \circ \phi_{s_{1}}^{Y_{1}}\right)(x)\right) \in \operatorname{Hol}\left(L_{y}^{E}\right) \subset \operatorname{Hol}\left(L_{x}^{E}\right) .
$$

Logo $y \in \operatorname{Hol}\left(L_{x}^{E}\right)$.

$\operatorname{Assim} L_{x} \subset \operatorname{Hol}\left(L_{x}^{E}\right)$.

Seja $\mathrm{P}_{\alpha}(y) \in \operatorname{Hol}\left(L_{x}^{E}\right)$, com $y \in L_{x}^{E}$ e $\alpha \in C_{\text {par. }}^{\infty}([0,1], D)$. Existem $m \in \mathbb{Z}_{+}^{*}, X \in$ $\mathfrak{X}\left(\mathcal{F}^{E}\right)^{m}$ e $t \in \mathbb{R}^{m}$, tais que $y=\phi_{t}^{X}(x)$. Ainda, dado que $\alpha=\alpha_{1} * \cdots * \alpha_{n}$, com $\alpha_{1}, \cdots, \alpha_{n} \in C^{\infty}\left([0,1], \mathcal{F}_{B}\right)$ regulares, existem $\widetilde{Y} \in \mathfrak{X}^{\mathrm{H}}(E)^{n}$ e $s \in \mathbb{R}^{n}$, tais que $\alpha_{i}$ é um segmento de curva integral de $Y_{i}$, para todo $i \in\{1, \cdots, n\}$.

$\operatorname{Logo} \mathrm{P}_{\alpha}(y)=\left(\phi_{s}^{\tilde{Y}} \circ \phi_{t}^{X}\right)(x) \in L_{x}^{\mathrm{H}}$.

$\operatorname{Assim} L_{x} \supset \operatorname{Hol}\left(L_{x}^{E}\right)$.

Motivando-se pelo item (b) do lema anterior, a folheação $\mathcal{F}\left(\mathrm{H}, \mathcal{F}^{E}\right)$ será denominada a folheação de holonomia generalizada gerada pela folheação $\mathcal{F}^{E}$ e pela distribuição horizontal H.

Quando $D$ é integrável, ou seja, quando $D=\mathrm{T} \mathcal{F}_{M}$, com $\mathcal{F}_{M}$ uma folheação (regular) de $M$, dado que $C_{\text {par. }}^{\infty}\left([0,1], \mathcal{F}_{M}\right):=C_{\text {par. }}^{\infty}\left([0,1], \mathrm{T} \mathcal{F}_{M}\right)$ é o conjunto das curvas suaves por partes inteiramente contidas em folhas de $\mathcal{F}_{M}$, tem-se que a projeção $\pi: E \longrightarrow M$ é folheada, como consequência imediata do item (b) do lema anteior.

Relembre, através da Proposição 2.18, que para cada $b \in B, \mathrm{~K}_{b}^{0}$ era a componente conexa maximal, em torno da identidade, do subgrupo de isometrias

$$
\operatorname{Iso}\left(\mathcal{F}_{b}\right):=\left\{T \in \operatorname{Iso}\left(E_{b}\right): T(L) \subset L, \forall L \in \mathcal{F}_{b}\right\}
$$

Analogamente ao que ocorre no Lema 4.16 , o grupo $\mathrm{K}_{b}^{0}$ (resp. $\overline{\mathrm{K}}_{b}^{0}$ ) age efetivamente sobre $E_{b}$, a saber, pela ação dada por $k \cdot x:=k(x)$ e, assim, a fibra $E_{b}$ é homogeneamente folheada 
pelas órbitas do grupo $\mathrm{K}_{b}^{0}$ (resp. $\overline{\mathrm{K}}_{b}^{0}$ ), de modo que $\left\{\mathrm{K}_{b}^{0}(x): x \in E_{b}\right.$ e $\left.b \in B\right\}$ (resp. $\left\{\overline{\mathrm{K}}_{b}^{0}(x)\right.$ : $x \in E_{b}$ e $\left.b \in B\right\}$ ) é uma partição de $E$, a qual será denotada por $\mathcal{F}^{\mathrm{K}}$ (resp. $\mathcal{F}^{\overline{\mathrm{K}}}$ ).

A seguir será mostrado que a partição $\mathcal{F}^{\mathrm{K}}$ (resp. $\mathcal{F}^{\overline{\mathrm{K}}}$ ), assim como a folheação infinitesimal $\mathcal{F}^{E}$, é Hol-invariante.

Lema 4.21) Dado $\alpha \in C_{\text {par. }}^{\infty}\left([0,1], \mathcal{F}_{M}\right)$, considere $\mathrm{C}_{\alpha}: \operatorname{Iso}\left(E_{\alpha(1)}\right) \longrightarrow \operatorname{Iso}\left(E_{\alpha(0)}\right)$ a aplicação dada por $\mathrm{C}_{\alpha}(T)=\mathrm{P}_{\alpha^{-1}} \circ T \circ \mathrm{P}_{\alpha}$. Então

a) $\mathrm{C}_{\alpha}\left(\mathrm{K}_{\alpha(1)}^{0}\right)=\mathrm{K}_{\alpha(0)}^{0}\left(\operatorname{resp} . \mathrm{C}_{\alpha}\left(\overline{\mathrm{K}}_{\alpha(1)}^{0}\right)=\overline{\mathrm{K}}_{\alpha(0)}^{0}\right)$,

b) (Hol-invariância) $\mathrm{P}_{\alpha}\left(\mathrm{K}_{\alpha(0)}^{0}(x)\right) \subset \mathrm{K}_{\alpha(1)}^{0}\left(\mathrm{P}_{\alpha}(x)\right)\left(\operatorname{resp} . \mathrm{P}_{\alpha}\left(\overline{\mathrm{K}}_{\alpha(0)}^{0}(x)\right) \subset \overline{\mathrm{K}}_{\alpha(1)}^{0}\left(\mathrm{P}_{\alpha}(x)\right)\right)$, para todo $x \in E$.

\section{Demonstração.}

a) Basta provar que $\mathrm{C}_{\alpha}\left(\mathrm{K}_{\alpha(1)}^{0}\right) \subset \mathrm{K}_{\alpha(0)}^{0}$, dado que isto implicaria que

$$
\mathrm{K}_{\alpha(1)}^{0} \subset \mathrm{C}_{\alpha^{-1}}\left(\mathrm{~K}_{\alpha(0)}^{0}\right) \subset \mathrm{K}_{\alpha(1)}^{0}
$$

Seja $k \in \operatorname{Iso}\left(\mathcal{F}_{\alpha(1)}\right)$. Então $\mathrm{C}_{\alpha}(k) \in \operatorname{Iso}\left(\mathcal{F}_{\alpha(0)}\right)$.

Uma vez que $\mathrm{P}_{\alpha}, \mathrm{P}_{\alpha^{-1}}$ são isometrias $\mathcal{F}^{E}$-folheadas e $k$ é uma isometria que fixa $\mathcal{F}_{\alpha(1)}$, tem-se que

$$
\mathrm{C}_{\alpha}\left(\operatorname{Iso}\left(\mathcal{F}_{\alpha(1)}\right)\right) \subset \operatorname{Iso}\left(\mathcal{F}_{\alpha(1)}\right)
$$

Mas $\mathrm{C}_{\alpha}: \mathbb{G L}\left(E_{\alpha(1)}\right) \longrightarrow \mathbb{G} L\left(E_{\alpha(0)}\right)$ é linear e, portanto, diferenciável.

Assim, como $\mathrm{K}_{\alpha(1)}^{0} \subset \operatorname{Iso}\left(\mathcal{F}_{\alpha(1)}\right)$ é um subconjunto conexo, segue que $\mathrm{C}_{\alpha}\left(\mathrm{K}_{\alpha(1)}^{0}\right) \subset$ Iso $\left(\mathcal{F}_{\alpha(0)}\right)$ é um subconjunto conexo e, dado que $\operatorname{Id}_{E_{\alpha(0)}} \in \mathrm{C}_{\alpha}\left(\mathrm{K}_{\alpha(1)}^{0}\right)$ e $\mathrm{K}_{\alpha(0)}^{0}$ é componente conexa de $\operatorname{Iso}\left(\mathcal{F}_{\alpha(0)}\right)$ em torno $\operatorname{Id}_{E_{\alpha(0)}}$, conclui-se que $\mathrm{C}_{\alpha}\left(\mathrm{K}_{\alpha(1)}^{0}\right) \subset \mathrm{K}_{\alpha(0)}^{0}$.

b) A afirmação segue diretamente do item anterior e do fato de que

$$
\mathrm{P}_{\alpha}(k(x))=\mathrm{P}_{\alpha}\left(k\left(\mathrm{P}_{\alpha^{-}}\left(\mathrm{P}_{\alpha}(x)\right)\right)\right)=\mathrm{C}_{\alpha^{-1}}(k)\left(\mathrm{P}_{\alpha}(x)\right),
$$

para todo $k \in \mathrm{K}_{\alpha(0)}^{0}\left(\right.$ resp. $\left.k \in \overline{\mathrm{K}}_{\alpha(0)}^{0}\right)$.

Analogamente ao que foi feito para a construção da folheação de holonomia generalizada $\mathcal{F}\left(\mathrm{H}, \mathcal{F}^{E}\right)$, pode-se considerar $\mathcal{F}^{\ell}\left(\mathrm{H}, \mathcal{F}^{E}\right):=\mathcal{F}\left(\mathrm{H}, \mathcal{F}^{\mathrm{K}}\right)\left(\operatorname{resp} . \mathcal{F}^{\mathrm{lc}}\left(\mathrm{H}, \mathcal{F}^{E}\right):=\mathcal{F}\left(\mathrm{H}, \mathcal{F}^{\overline{\mathrm{K}}}\right)\right)$ a folheação singular de $E$ obtida pela aplicação do Teorema de Sussmann (Teorema 1.12) ao subconjunto de campos vetoriais $\mathfrak{X}^{\mathrm{H}}(E) \cup \mathfrak{X}\left(\mathcal{F}^{\mathrm{K}}\right) \subset \mathfrak{X}(E)$ (resp. $\mathfrak{X}^{\mathrm{H}}(E) \cup \mathfrak{X}\left(\mathcal{F}^{\overline{\mathrm{K}}}\right) \subset \mathfrak{X}(E)$, onde $\mathfrak{X}^{\mathrm{H}}(E):=\left\{\widetilde{X} \in \mathfrak{X}(E): X \in \mathfrak{X}\left(\mathcal{F}_{B}\right)\right\}$. A saber, a folha de $\mathcal{F}^{\ell}\left(\mathrm{H}, \mathcal{F}^{E}\right)$ passando por $x \in E$ 
é dada por

$$
\begin{gathered}
L_{x}^{\ell}=\left\{\phi_{t}^{\tilde{X}}(x) \in E: n \in \mathbb{Z}_{+}^{*}, \tilde{X} \in \mathfrak{X}^{\mathrm{H}}(E)^{n} \cup \mathfrak{X}\left(\mathcal{F}^{\mathrm{K}}\right)^{n}, t \in I^{\tilde{X}} \subset \mathbb{R}^{n}\right\} \\
\left(\text { resp. } L_{x}^{\text {lc }}=\left\{\phi_{t}^{\widetilde{X}}(x) \in E: n \in \mathbb{Z}_{+}^{*}, \tilde{X} \in \mathfrak{X}^{\mathrm{H}}(E)^{n} \cup \mathfrak{X}\left(\mathcal{F}^{\overline{\mathrm{K}}}\right)^{n}, t \in I^{\tilde{X}} \subset \mathbb{R}^{n}\right\}\right) .
\end{gathered}
$$

A Hol-invariância da partição $\mathcal{F}^{\mathrm{K}}$ (resp. $\mathcal{F}^{\overline{\mathrm{K}}}$ ), vista no lema anterior, garantirá que a folheação $\mathcal{F}^{\ell}\left(\mathrm{H}, \mathcal{F}^{E}\right)$ (resp. $\mathcal{F}^{\mathrm{lc}}\left(\mathrm{H}, \mathcal{F}^{E}\right)$ ) possuirá uma caracterização semelhante a da folheação $\mathcal{F}\left(\mathrm{H}, \mathcal{F}^{E}\right)$, apresentadas no item (b) Lema 4.20 , como pode ser visto a seguir.

Lema 4.22) Seja $x \in E, \operatorname{com} \pi(x)=p$. Então
a) $\operatorname{Hol}\left(\mathrm{K}_{\pi(y)}^{0}(y)\right) \subset \operatorname{Hol}\left(\mathrm{K}_{p}^{0}(x)\right)$, para todo $y \in \operatorname{Hol}\left(\mathrm{K}_{p}^{0}(x)\right)$,
b) $\operatorname{Hol}\left(\overline{\mathrm{K}}_{\pi(y)}^{0}(y)\right) \subset \operatorname{Hol}\left(\overline{\mathrm{K}}_{p}^{0}(x)\right)$, para todo $y \in \operatorname{Hol}\left(\overline{\mathrm{K}}_{p}^{0}(x)\right)$,
c) $L_{x}^{\ell}=\operatorname{Hol}\left(\mathrm{K}_{p}^{0}(x)\right)$ e $\left.L_{x}^{\mathrm{lc}}=\operatorname{Hol}\left(\overline{\mathrm{K}}_{p}^{0}(x)\right)\right)$.

Demonstração. Análoga a demonstração do Lema 4.20.

\subsubsection{Teorema C}

Será apresentado, nesta breve seção, o enunciado preciso e a prova do Teorema $\mathrm{C}$ descrito na introdução e no início deste capítulo e para este fim, faz-se necessário estabelecer, brevemente, alguma notação de modo a considerar a folheação de holonomia generalizada $\mathcal{F}\left(\mathrm{H}, \mathcal{F}^{E}\right)$ bem como as folheações $\mathcal{F}^{\ell}\left(\mathrm{H}, \mathcal{F}^{E}\right)$ e $\mathcal{F}^{\mathrm{lc}}\left(\mathrm{H}, \mathcal{F}^{E}\right)$ (folheações modelo apresentadas na seção anterior) no contexto apropriado de folheações singulares.

Sejam $(M, g)$ uma variedade Riemanniana, $\mathcal{F}$ uma FRS, $B \subset M$ subvariedade fechada, saturada e contida em um estrato.

Dado $W=\exp \left(\nu^{\varepsilon} B\right)$ uma $\varepsilon$-VTD (em torno de $B$ ), relembre da seção 4.1, que era possível tomar $\mathcal{T} \subset \mathrm{T} \mathcal{F}_{W}$ uma extensão de $\mathrm{T} \mathcal{F}_{B}$, de modo que a linearização $\mathcal{T}^{\ell}$ de $\mathcal{T}$ gerava uns distribuição horizontal $\mathrm{H}=\mathrm{H}^{\mathcal{T}} \subset \mathrm{T}(\nu B)$, ao longo de $\mathrm{T} \mathcal{F}_{B}$. A saber, $\mathrm{H}^{\mathcal{T}}$ era a extensão homotética de $\exp ^{*} \mathcal{T}^{\ell}$.

Dado $b \in B$, relembre da seção 2.2 , que a extensão homotética de $\left.\left(\exp _{b}\right)^{*} \mathcal{F}\right|_{W_{b}}$ era uma folheação infinitesimal de $\nu_{b} B$ denotada por $\mathcal{F}_{b}$, cuja folha passando por $x \in \nu_{b} B$ era denotada por $L_{x}^{b}$. Observe que

$$
\mathcal{F}^{\nu B}=\left\{L^{b}: L^{b} \in \mathcal{F}_{b}, b \in B\right\}
$$

é uma partição de $\nu B$ que é fibra à fibra é uma folheação infinitesimal. 
Ainda, relembre desta mesma seção, que a componente conexa maximal, em torno da identidade, do grupo Iso $\left(\mathcal{F}_{b}\right)$ de isometrias de $\left(\nu_{b} B, g_{b}\right)$ que fixam $\mathcal{F}_{b}$, era um subgrupo de Lie denotado por $K_{b}^{0}$.

Proposição 4.23) (Roda e translada) Seja $X \in \mathfrak{X}\left(\mathcal{F}_{W}\right)$ e denote por $\phi^{X^{\ell}}: D^{X^{\ell}} \subset \mathbb{R} \times$ $\nu B \longrightarrow \nu B$ o fluxo da linearização de $X$. Então $\phi_{t}^{X^{\ell}}(x) \in \operatorname{Hol}\left(\mathrm{K}_{\pi(x)}^{0}(x)\right)$, para todo $(t, x) \in D^{X^{\ell}}$.

Demonstração. Tome $P \subset B$ uma placa normal em torno de $\pi(x)=b$. Existe $a \in \mathbb{R}$, tal que $x \in D_{t}$ e $b_{t}:=\pi\left(\phi_{t}^{X^{\ell}}(x)\right)=\phi_{t}^{X^{\ell}}(b) \in P$, para todo $t \in[0, a]$. Considere, para cada $t \in[0, a]$, o único segmento de geodésica $\gamma_{b_{t}, b}$, em $P$, ligando $b_{t}$ a $b$.

Defina $k:[0, a] \subset \mathbb{R} \longrightarrow \mathbb{G} \mathbb{L}\left(\nu_{b} B\right)$ por $k(t):=\left.\mathrm{P}_{\gamma_{b_{t}, b}} \circ \phi_{t}^{X^{\ell}}\right|_{\nu_{b} B}$. Então

- $k(0)=\mathrm{Id}$,

- $k$ é contínua, pois $t \longmapsto \mathrm{P}_{\gamma_{b_{t}, b}}$ e $\left.t \longmapsto \phi_{t}^{X^{\ell}}\right|_{\nu_{b} B}$ são curvas suaves em $\mathbb{G} \mathbb{L}(\nu B)$ (grupóide linear geral de $\nu B$ ) e $k$ é dada pela multiplicação dessas duas curvas,

- $\operatorname{Im}(k) \subset \operatorname{Iso}\left(\nu_{b} B\right)$, pois $\mathrm{P}_{\gamma_{b_{t}, b}}$ é uma isometria, dado que $\mathcal{T}^{\ell}$ é compatível com a métrica $\left.g\right|_{\nu B}$, e $\left.\phi_{t}^{X^{\ell}}\right|_{\nu_{b} B}$ é uma isometria, como visto na Proposição 2.15,

- $k(t)$ fixa $\mathcal{F}_{b}$, para todo $t \in[0, a]$, pois $\mathrm{P}_{\gamma_{b_{t}, b}}$ fixa $\mathcal{F}_{b}$, dado que $\mathcal{T}^{\ell} \subset \mathrm{T} \mathcal{F}_{W}$, e $\left.\phi_{t}^{X}\right|_{\nu_{b} B}$ fixa $\mathcal{F}_{b}$, dado que $X^{\ell} \in \mathfrak{X}\left(\mathcal{F}_{W}\right)$.

Segue que $k$ é uma curva na componente conexa de $\operatorname{Iso}\left(\mathcal{F}_{b}\right)$, em torno de $\operatorname{Id}$, ou seja, $\operatorname{Im}(k) \subset$ $\mathrm{K}_{b}^{0}$.

$\operatorname{Logo} \mathrm{P}_{\gamma_{b_{t}, b}}\left(\phi_{t}^{X^{\ell}}(x)\right)=k(t) \cdot x \in \mathrm{K}_{b}^{0}(x)$, ou seja,

$$
\phi_{t}(x) \in \operatorname{Hol}\left(\mathrm{K}_{b}^{0}(x)\right) \text {, }
$$

para todo $t \in[0, a]$.

Assim, como $L_{b}$ pode ser coberta por placas normais e $\pi\left(\phi_{t}(x)\right) \in L_{b}$, para todo $t \in I^{X^{\ell}} \subset \mathbb{R}$, pelo item (a) do Lema 4.20, segue que $\phi_{t}(x) \in \operatorname{Hol}\left(\mathrm{K}_{b}^{0}(x)\right)$, para todo $t \in I^{X^{\ell}} \subset \mathbb{R}$.

Teorema 4.24) Seja $(M, g)$ uma variedade Riemanniana, $\mathcal{F}$ uma FRS e $B \subset M$ uma subvariedade fechada, saturada e contida em um extrato.

Então, existem $W$ uma $\varepsilon$-VTD, em torno de $B$, e $\mathcal{T} \subset \mathrm{T} \mathcal{F}_{W}$ uma extensão de $\mathrm{T} \mathcal{F}_{B}$, tais que $\left.\exp \right|_{\nu^{\varepsilon} B}: \nu^{\varepsilon} B \longrightarrow W$ é difeomorfismo folheado entre

a) $\mathcal{F}\left(\mathcal{T}^{\ell}, \mathcal{F}^{\nu B}\right)$ e $\mathcal{F}_{W}$,

b) $\mathcal{F}^{\ell}\left(\mathcal{T}^{\ell}, \mathcal{F}^{\nu B}\right)$ e $\mathcal{F}^{\ell}$,

c) $\mathcal{F}^{\text {lc }}\left(\mathcal{T}^{\ell}, \mathcal{F}^{\nu B}\right)$ e $\mathcal{F}^{\text {lc }}$.

Demonstração. Considere $W$ uma vizinhança tubular saturada, em torno de $B$, e $\mathcal{T} \subset$ $\mathrm{T}_{\mathcal{F}}$ uma distribuição (regular) que extende $\mathrm{TF}_{B}$, tal que $\mathcal{T} \cap \operatorname{Ker}(\mathrm{d} \rho)=\{0\}$ (ver Proposição 4.3, para prova da existência). Ainda, ao longo da demonstração, considere fixo $x \in \exp ^{-1}(W)$, $\operatorname{com} p=\exp (x) \in W$ e $\pi(x)=\rho(p)=b$. 
a) Seja $y=\mathrm{P}_{\alpha}(z) \in \operatorname{Hol}\left(L_{x}^{b}\right)$, com $z \in L_{x}^{b}$ e $\alpha \in C_{\text {par. }}^{\infty}\left([0,1], \mathcal{F}_{B}\right)$, tal que $\alpha(0)=b$. Observe que $\exp (z) \in L_{\exp (x)} \cap W_{b}$ e, em particular, $L_{\exp (z)}=L_{p}$.

Tome $\tilde{\alpha}_{z}:[0,1] \subset \mathbb{R} \longrightarrow \nu B$ o levantamento $\mathcal{T}^{\ell}$-horizontal de $\alpha$ com relação a $z$, a saber, dado por $\tilde{\alpha}_{z}(t)=\mathrm{P}_{\alpha}^{t}(z)$.

Uma vez que que $\mathcal{T}^{\ell}$ é tangente a extensão homotética de exp ${ }^{*} \mathcal{F}$, segue que $\tilde{\alpha}_{z}$ está contida na folha desta extensão, passando por $z=\tilde{\alpha}_{z}(0)$. Logo, como $z \in \exp ^{-1}(W)$, tem-se que $\operatorname{Im}\left(\tilde{\alpha}_{z}\right) \subset \exp ^{-1}\left(L_{\exp (z)}\right)=\exp ^{-1}\left(L_{p}\right)$ e, em particular,

$$
y=\tilde{\alpha}_{z}(1) \in \exp ^{-1}\left(L_{p}\right)
$$

Assim

$$
\operatorname{Hol}\left(L_{x}^{b}\right) \subset \exp ^{-1}\left(L_{p}\right)
$$

Seja $y=\exp ^{-1}(q) \in \exp ^{-1}\left(L_{p}\right)$, com $q \in L_{p}$. Observe que $L_{\rho(q)}=L_{b}$, uma vez que $L_{p}=L_{q}$ e $\rho$ é folheada.

Tome $\beta \in C^{\infty}\left([0,1], \mathcal{F}_{B}\right)$ ligando $\rho(q)$ a $b$, e considere $\tilde{\beta}_{y}:[0,1] \subset \mathbb{R} \longrightarrow \nu B$ o levantamento $\mathcal{T}^{\ell}$-horizontal de $\beta$ com relação a $y$, a saber, dado por $\tilde{\beta}_{y}(t)=\mathrm{P}_{\beta}^{t}(y)$.

Uma vez que que $\mathcal{T}^{\ell}$ é tangente a extensão homotética de $\exp ^{*} \mathcal{F}$, segue que $\tilde{\beta}_{y}$ está contida na folha desta extensão, passando por $y=\tilde{\beta}_{y}(0)$ e, como $y \in \exp ^{-1}(W)$, tem-se que $\operatorname{Im}\left(\tilde{\beta}_{y}\right) \subset \exp ^{-1}\left(L_{\exp (y)}\right)=\exp ^{-1}\left(L_{p}\right)$ e, em particular,

$$
\mathrm{P}_{\beta}(y)=\tilde{\beta}_{y}(1) \in \exp ^{-1}\left(L_{p}\right) \text {. }
$$

Logo, dado que $\mathrm{P}_{\beta}(y) \in \exp ^{-1}\left(W_{b}\right)$, conclui-se que

$$
\mathrm{P}_{\beta}(y) \in \exp ^{-1}\left(L_{p} \cap W_{b}\right)=L_{x}^{b}
$$

Assim

$$
\operatorname{Hol}\left(L_{x}^{b}\right) \supset \exp ^{-1}\left(L_{p}\right)
$$

b) Seja $y=\mathrm{P}_{\alpha}\left(k_{b}(x)\right) \in \operatorname{Hol}\left(\mathrm{K}_{b}^{0}(x)\right)$, com $k_{b} \in \mathrm{K}_{b}^{0}$ e $\alpha \in C_{\text {par. }}^{\infty}\left([0,1], \mathcal{F}_{B}\right)$, tal que $\alpha(0)=b$. Tome $\tilde{\alpha}:[0,1] \subset \mathbb{R} \longrightarrow \nu^{\varepsilon} B$ o levantamento $\mathcal{T}^{\ell}$-horizontal de $\alpha$ com relação a $k_{b}(x)$, a saber, dado por $\tilde{\alpha}(t)=\mathrm{P}_{\alpha}^{t}\left(k_{b}(x)\right)$.

Uma vez que que $\mathcal{T}^{\ell}$ é tangente a extensão homotética de $\exp ^{*} \mathcal{F}^{\ell}$, segue que $\tilde{\alpha}$ está contida na folha desta extensão, passando por $k_{b}(x)=\tilde{\alpha}(0)$ e, como $k_{b}(x) \in \exp ^{-1}(W)$, tem-se que $\operatorname{Im}(\tilde{\alpha}) \subset \exp ^{-1}\left(L_{\exp \left(k_{b}(x)\right)}^{\ell}\right)$ e, em particular,

$$
y \in \exp ^{-1}\left(L_{\exp \left(k_{b}(x)\right)}^{\ell}\right)
$$


Mas, pela Proposição 2.22, tem-se que $\exp \left(\mathrm{K}_{b}^{0}(x)\right)=L_{p}^{\ell} \cap W_{b}$, de modo que $\exp \left(k_{b}(x)\right) \in$ $L_{p}^{\ell} \cap W_{b}$ e, portanto, $L_{\exp \left(k_{b}(x)\right)}^{\ell}=L_{p}^{\ell}$. Logo $y \in L_{p}^{\ell}$.

Assim

$$
\operatorname{Hol}\left(\mathrm{K}_{b}^{0}(x)\right) \subset \exp ^{-1}\left(L_{p}^{\ell}\right)
$$

Seja $y=\exp ^{-1}(q) \in \exp ^{-1}\left(L_{p}^{\ell}\right), \operatorname{com} q \in L_{p}^{\ell}$. Existem $n \in \mathbb{Z}_{+}^{*}, X \in \mathfrak{X}\left(\mathcal{F}_{W}\right)^{n}$ e $t \in \mathbb{R}^{n}$, tais que

$$
y=\phi_{t}^{X^{\ell}}(x)
$$

Mas, por indução, tem-se que que $\phi_{t}^{X^{\ell}}(x) \in \operatorname{Hol}\left(\mathrm{K}_{b}^{0}(x)\right)$, para todo $n \in \mathbb{Z}_{+}^{*}, X \in \mathfrak{X}\left(\mathcal{F}_{W}\right)^{n}$ e $t \in \mathbb{R}^{n}$. De fato

- se $n=1$, pelo Proposição 4.23 , tem-se que $\phi_{t}^{X^{\ell}}(x) \in \operatorname{Hol}\left(\mathrm{K}_{b}^{0}(x)\right)$;

- se a afirmação é válida para $k \in \mathbb{Z}_{+}^{*}$, denotando-se $z=\phi_{t}^{X^{\ell}}(x)$ segue, pela Proposição 4.23, que

$$
\phi_{t_{(k+1)}}^{X_{(k+1)}^{\ell}}\left(\phi_{t}^{X^{\ell}}(x)\right) \in \operatorname{Hol}\left(\mathrm{K}_{\pi(z)}^{0}(z)\right)
$$

e, pelo Lema 4.22 , tem-se que $\operatorname{Hol}\left(\mathrm{K}_{\pi(z)}^{0}(z)\right) \subset \operatorname{Hol}\left(\mathrm{K}_{b}^{0}(x)\right)$ e, portanto

$$
\phi_{t_{(k+1)}}^{X_{(k+1)}^{\ell}}\left(\phi_{t}^{X^{\ell}}(x)\right) \in \operatorname{Hol}\left(\mathrm{K}_{b}^{0}(x)\right)
$$

Assim

$$
\operatorname{Hol}\left(\mathrm{K}_{b}^{0}(x)\right) \supset \exp ^{-1}\left(L_{p}^{\ell}\right)
$$

c) Segue de maneira análoga ao item anterior. 


\section{Referências Bibliográficas}

[1] ALEXANDRINO, M. M. Desingularization of singular Riemannian foliation. Geometriae Dedicata, v. 149, p. 397-416, mar. 2010.

[2] ALEXANDRINO, M. M. Proofs of conjectures about singular Riemannian foliations. Geometriae Dedicata, v. 119, p. 219-234, may 2006.

[3] ALEXANDRINO, M. M. ; BETTIOL, R. G. Lie groups and geometric aspects of isometric actions. New York: Springer, 2015.

[4] AlEXAndrino, M. M.; BRIQUet, R.; TÖBEN, D. Progress in the theory of singular Riemannian foliations. Differential Geometry and its Applications, v. 31, n. 2, p. 248-267, apr. 2013.

[5] ALEXANDRINO, M. M.; INAGAKI, M. K.; STRUCHINER, I. Lie groupoids and semilocal models of singular Riemannian foliations. ArXiv : 1812.03614, dec. 2018. Disponível em: https://arxiv.org/pdf/1812.03614.pdf. Acesso em: 6 set. 2020.

[6] ALEXANDRINO, M. M.; LYTCHAK, A. On smoothness of isometries between orbit spaces. Riemannian Geometry and Applications - Proceedings RIGA, Bucharest, p. 17-28, 2011.

[7] ALEXANDRINO, M. M.; RADESCHI, M. Closure of singular foliations: the proof of Molinos conjecture. Compositio Mathematica, v. 153, n. 12, p. 2577-2590, dec. 2017.

[8] ALEXANDRINO, M. M.; RADESCHI, M. Smoothness of isometric flows on orbit spaces and applications to the theory of foliations. Transformation Groups, v. 22, p. 1-27, mar. 2017.

[9] BOLTON, J. Transnormal systems. The Quarterly Journal of Mathematics, v. 24, p. 385-395, jan. 1973.

[10] CAMACHO, C.; LINS NETO, A. Teoria geométrica das folheações. 2. ed., Rio de Janeiro: IMPA, 2019.

[11] CARVAlHO, F. S. Orbifolds como grupóides e o grupo fundamental de um orbifold. 2015. Dissertação (Mestrado em Matemática) - Departamento de Matemática, Universidade Federal do Paraná, Curitiba, 2015.

[12] CRAINIC, M.; FERNANDES, R. L. Lectures on integrability of Lie brackets. Geometry and topology monographs, v. 17, p. 1-107, apr. 2011.

[13] DUISTERMAAT, J. J.; KOLK, J. A. C. Lie groups. Berlin: Springer-Verlag, 2000. 
[14] HIRSCH, M. W. Differential topology. New York: Springer-Verlag, 1976. (Graduate texts in mathematics, v. 33).

[15] GONZÁLES, A. G. G. Groupoids and singular foliations. 2019. Dissertation (Doctor of Science) - Faculty of Science, Katholieke Universiteit Leuven, Leuven, 2019.

[16] KOLÁR̆, I; MICHOR, P. W.; SLOVÁK, J. Natural Operations in Differential Geometry. New York: Springer-Verlag, 1993.

[17] KOBAYASHI, S.; NOMIZU, K. Foundations of differential geometry. New York: John Wiley \& Sons, 1963. v. 1.

[18] MACKENZIE, K. Lie groupoids and Lie algebroids in differential geometry. New York: Cambridge University Press, 1987. (London mathematical society-lecture notes series v. 124).

[19] MARLE, C. M. Calculus on Lie algebroids, Lie groupoids and Poisson manifolds. Dissertationes Mathematicae, v. 457, p. 1-57, jun. 2008.

[20] MARTIN, L. A. B. S. Grupos de Lie. Campinas: Editora Unicamp, 2016.

[21] MENDES, R.; RADESCHI, M. A slice theorem for singular Riemannian foliations, with applications. Transactions of American Mathematical Society, v. 371, n. 7, p. 49314949, nov. 2018.

[22] MOERDIJK, I.; MRČUN, J. Introduction to foliations and Lie groupoids. Cambridge: Cambridge University Press, 2003.

[23] MOLINO, P. Riemannian foliations. Boston: Birkhäuser, 1988. (Progress in mathematics, v. 73)

[24] MOLINO, P. Orbit-like foliations. Geometric Study of Foliations - Proceedings of the International Symposium/Workshop, Singapore, p. 97-119, dec. 1994.

[25] MORITA, S. Geometry of differential forms. Providence: American Mathematical Society, 2001. (Translations of mathematical monographs, v. 201).

[26] MUKHERJEE, A. Differential topology. $2^{\text {nd }}$ ed. Basel: Birkhäuser, 2015.

[27] O'NEILL, B. Semi-Riemannian geometry: with applications to relativity. San Diego: Academic Press, 1983. (Pure and applied mathematics).

[28] PETERSEN, P. Riemannian geometry. $3^{\text {rd }}$ ed. [S. 1.]: Springer International Publishing, 2016. (Graduate texts in mathematics, 171).

[29] PIÑEROS, D. A. C. Sobre as folheações e o teorema de slice para folheações Riemannianas singulares com seções. 2008. Dissertação (Mestrado em Ciências) Instituto de Matemática e Estatística, Universidade de São Paulo, São Paulo, 2008. 
[30] RADESCHI, M. Lecture notes on singular Riemannian foliations. 2017. Disponível em: https://static1.squarespace.com/static/5994498937c5815907f7eb12/t/ 5998477717bffc656afd46e0/1503151996268/SRF+Lecture+Notes.pdf. Acesso em: 5 mar. 2020.

[31] STEFAN, P. Accessible sets, orbits, and foliations with singularities. Proceedings of London Mathematical Society, v. 29, n. 3, p. 699-713, dec. 1974.

[32] SUSSMANN, H. J. Orbits of families of vector fields and integrability of distributions. Transactions of American Mathematical Society, v. 180, p. 171-188, 1973.

[33] TU, L. W. Differential geometry: connections, curvature, and characteristic classes. [S. 1.]: Springer International Publishing, 2017. (Graduate texts in mathematics, 275). 